\title{
REALIZING THE POTENTIAL OF PROTEIN-PROTEIN INTERACTION PREDICTION FOR STUDYING SINGLE AND EVOLUTIONARILY SIMILAR ORGANISMS \\ $\&$ \\ ENGINEERING INHIBITORY PROTEINS WITH INSIPS: THE IN SILICO PROTEIN SYNTHESIZER
}

by

Andrew Schoenrock

Submitted in partial fulfillment of the requirements for the degree of

Doctor of Philosophy in Computer Science

at

Carleton University

Ottawa, Ontario

January 2016

(c) Copyright by Andrew Schoenrock, 2016 


\section{Abstract}

Protein-protein interactions (PPIs) play a vital role in the life cycle of a cell and the elucidation of PPIs and PPI networks have become the interest of many over the past 15 years. There are a variety of experimental techniques to determine PPIs, however they are expensive, time and resource consuming and have relatively high error rates. To combat this, many computational tools have been produced to predict PPIs. These tools, however, have their own downfalls, including limited applicability, lack of supporting biological data, long running times and inadequately high error rates. The Protein-protein Interaction Prediction Engine (PIPE) is a computational tool used to predict PPIs which overcome the limitations of other methods and is able to perform proteome-wide PPI predictions within a wide range of organisms with a very low false positive rate $(<0.05 \%)$ in a reasonable amount of time.

This thesis has four main areas of contribution. First, extensions to the PIPE method (including a method to predict PPI sites within a protein pair and a new scoring function used to determine PPIs) and performance improvements over the last PIPE implementation will be detailed. Secondly, the study of PIPE predicted PPIs will be used to produce novel biological insights in S. cerevisiae and H. Sapiens and the capability of PIPE to produce cross-species PPIs will be shown. Next, PIPE will be used to study the evolution of two families of organisms, starting with a family of green algae containing members at different stages of the transition from unicellularity to multicellularity followed by a group of five closely related yeast strains. In the latter, a novel null model used to compare PPI networks will also be introduced. Lastly, the PIPE method will be incorporated with a genetic algorithm to create a massively parallel computational tool used to generate novel, synthetic protein sequences designed to interact with a specific target while avoiding off-target interactions. Proteins with these properties can be used in an inhibitory manner and can form the basis for the development of various therapeutics. Wet-lab experiments were done to verify the generated proteins' function in multiple cases. 


\section{Table of Contents}

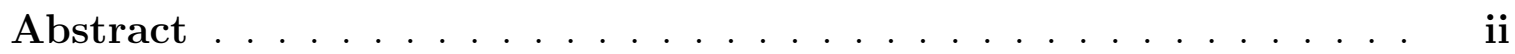

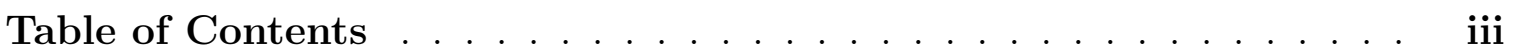

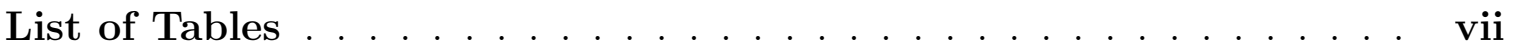

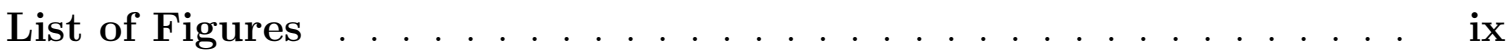

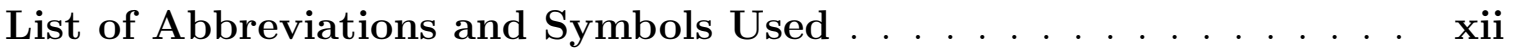

Chapter 1 Introduction $\ldots \ldots \ldots \ldots \ldots$

1.1 Summary of Contributions . . . . . . . . . . . . . 3

Chapter 2 Protein-Protein Interaction Prediction Methods Review 8

2.1 Methods Based on Genomic Information . . . . . . . . . . . . . . 8

2.2 Methods Based on Evolutionary Relationships . . . . . . . . . . . . 10

2.3 Methods Based on 3D Protein Structure . . . . . . . . . . . . . 13

2.4 Methods Based on Network Structure . . . . . . . . . . . . . . 17

2.5 Methods Based on Protein Domains . . . . . . . . . . . . . . . . . 21

2.6 Methods Based on Machine Learning Techniques _ . . . . . . . . 24

2.6.1 Subclass of Approaches Based on Primary Protein Structure

Alone . . . . . . . . . . . . . . . . . . . . 27

Chapter 3 History of PIPE and MP-PIPE $\ldots \ldots \ldots \ldots$

3.1 PIPE . . . . . . . . . . . . . . . . . . . . 30

3.1.1 The Main PIPE Algorithm . . . . . . . . . . . . . . . 30

3.1.2 PIPE Version 1 Overall Results . . . . . . . . . . . . . . 32

Ability of PIPE to correctly identify interacting and non-interacting

pairs . . . . . . . . . . . . . . . 32

Ability of PIPE to Detect Interaction Sites Between Interacting

Protein Pairs . . . . . . . . . . . . . . . 32

Ability of PIPE to Detect Novel Protein-Protein Interactions . 34

Ability of PIPE to Elucidate the Internal Architecture of Protein Complexes . . . . . . . . . . . . . . 35 


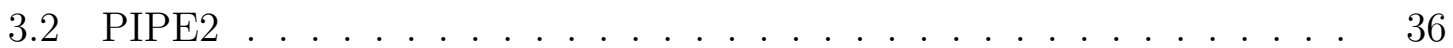

3.2.1 Improving PIPE's Running Time . . . . . . . . . . . . . . 37

3.2.2 Improving PIPE's Predictive Performance . . . . . . . . . . . 40

3.2.3 Leave-One-Out Cross-Validation tests . . . . . . . . . . . . . . 40

3.2.4 PIPE2 Overall Results . . . . . . . . . . . . . . . . . . . 43

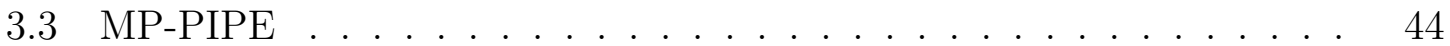

3.3.1 New Parallel Architecture of MP-PIPE . . . . . . . . . . . . 45

3.3.2 MP-PIPE Benchmarking . . . . . . . . . . . . . . . . . 47

Benchmarking Test 1 Results . . . . . . . . . . . . . . . 48

Benchmarking Test 2 Results . . . . . . . . . . . . . . 50

3.3.3 Summary of Biological Results Produced by MP-PIPE . . . . 50

Caenorhabditis elegans Proteome-wide prediction results . . . 50

Homo sapiens Proteome-wide prediction results . . . . . . . . 52

Chapter 4 Further Development and Improvements of PIPE . . . 55

4.1 PIPE-Sites . . . . . . . . . . . . . . . . . . 55

4.1.1 PIPE-Sites Algorithm, Initial Testing and Initial Results . . . 56

PIPE-Sites Algorithm . . . . . . . . . . . . . . 56

PIPE-Sites Evaluation . . . . . . . . . . . . . 57

4.1.2 Experimental Validation of Novel PIPE-Sites Predictions . . . 61

4.2 Further Computational Acceleration \& Modified Score Function . . . 62

4.2.1 PIPE Algorithm Modification, Data Structure Optimization \&

Performance Improvements _ . . . . . . . . . . . . 62

4.2 .2 Modified Score Function . . . . . . . . . . . . . . . . . 65

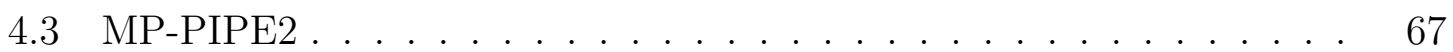

Chapter 5 Applications of PIPE \& Analysis of Resulting Data . . 69

5.1 Cross-species PPI prediction using MP-PIPE . . . . . . . . . . 69

5.1.1 Application of MP-PIPE to a variety of organisms . . . . . . 70

5.1.2 Using MP-PIPE to produce cross-species PPI predictions . . . 72

5.1.3 Using a compilation of interactions from a set of different organisms to make PPI predictions . . . . . . . . . . . . 74

5.1.4 Independent comparison of PIPE to other methods . . . . . . 74

5.2 Analysis of S. cerevisiae predicted PPI data used to infer novel biological information . . . . . . . . . . . . . . 77

5.3 In Depth Analysis of the Predicted H. sapiens Interactome . . . . . 78

5.3.1 Adding Credibility to the Novel prediction made by PIPE . . 78

PIPE predictions compared to new large-scale PPI detection experiments . . . . . . . . . . . . . 78 
PIPE Prediction Specificity when dealing with proteins with high sequence similarity . . . . . . . . . .

5.3.2 Network-wide analysis of high degree and high betweenness centrality proteins . . . . . . . . . . . . 80

5.3.3 Identification of potential protein complexes within the predicted H. sapiens interaction network . . . . . . . . . . . 83

5.3.4 Demonstrating the Value of the Predicted Interactome . . . . 86 Using the Predicted Human Protein Interaction Network to Identify Breast Cancer Related Proteins . . . . . . . 86

Identification of Novel Molecular Markers for Seasonal Allergic Rhinitis . . . . . . . . . . . . 87

Identification of Novel Proteins Involved in Translation . . . . 88

\section{Chapter 6 Using Predicted PPI Networks to Gain Insights into the Evolution of Closely Related Organisms . . . . . . . . 89}

6.1 Identifying Potential Protein Clusters within a PPI Network . . . . 90

6.2 Studying the Evolutionary Transition in Individuality of Multicellularity in the Volvocine Lineage of Green Algae using Predicted PPI Networks . . . . . . . . . . . . . . . . . . . 6.2.1 Computational Setup, Initial Data Sets \& Predicted PPI Networks . . . . . . . . . . . . . . . . . . 97

6.2 .2 Specificity Validation . . . . . . . . . . . . . . . . . . . 998

6.2.3 Results and Discussion . . . . . . . . . . . . . . . . . . 99

6.3 The Study of Closely Related Yeast Strains \& the Introduction of a Novel Null Model for Comparing Predicted PPI Networks . . . . . . . 106

6.3 .1 Wild Type Data . . . . . . . . . . . . . . . . . . . 107

6.3.2 Simulated Genomes \& the Construction of a Predicted PPI

Network Null Model . . . . . . . . . . . . . . . . . . . . . . 108

6.3.3 Methods of Network Comparison . . . . . . . . . . . . . . . . 110

Sequence and Gene-centered Analysis . . . . . . . . . . . 111

Quantification of Changes in the PPI Network . . . . . . . . 111

Species-Specific Interaction Analysis _. . . . . . . . . . . 112

Species-Specific Protein Cluster Analysis . . . . . . . . . . . 113

Gene Ontology (GO) Analysis . . . . . . . . . . . . . . . . 113

6.3.4 Results . . . . . . . . . . . . . . . . . . . . . . . . . 114

Changes in PPIs Across the Phylogeny . . . . . . . . . . . 115

Comparison of Molecular Evolution Using Sequence and PPIbased Methods . . . . . . . . . . . . . . 115

PPI-based Analysis . . . . . . . . . . . . . . . . . . . 123

Protein Cluster-based Analysis _. . . . . . . . . . . . 125

6.4 Conclusion . . . . . . . . . . . . . . . . . . . . 130 
Chapter 7 Engineering Inhibitory Proteins with InSiPS: The InSilico Protein Synthesizer . . . . . . . . . . . . 132

7.1 Introduction . . . . . . . . . . . . . . . . . . . . . . 132

7.2 The InSiPS Genetic Algorithm . . . . . . . . . . . . . . . 135

7.3 The InSiPS Fitness Function . . . . . . . . . . . . . . . . 137

7.4 The InSiPS Implementation $\ldots \ldots \ldots \ldots$

7.5 InSiPS Performance Testing and Benchmarking . . . . . . . . 147

7.5.1 Benchmarking Test 1: Number of Compute Threads Used . . 147

7.5.2 Benchmarking Test 2: Number of Slave Processes Used . . . 150

7.6 Experimental Validation on Real Problems . . . . . . . . . . . . . 154

7.6.1 InSiPS Parameter Tuning . . . . . . . . . . . . . . . . 154

7.6.2 Wet-Lab Experimental Validation . . . . . . . . . . . . . . 159

Target: YBL051C . . . . . . . . . . . . . . . . . . 162

Target: YAL017W . . . . . . . . . . . . . . 164

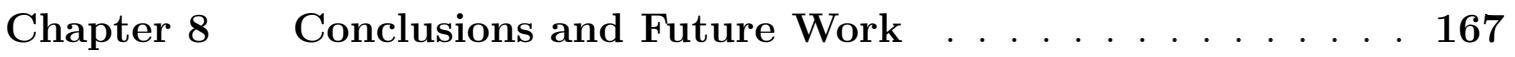

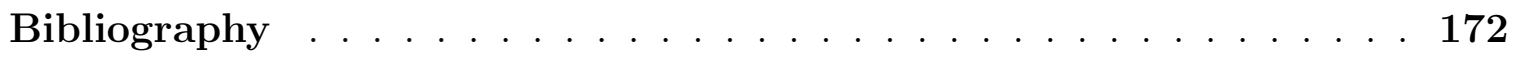




\section{List of Tables}

3.1 Performance improvements from PIPE1 to PIPE2. . . . . . . . 39

3.2 Analysis of PIPE2 prediction data. . . . . . . . . . . 44

4.1 PIPE-Sites interaction site prediction errors (average DM) for $S$. cerevisiae pairs, H. sapiens pairs compared to random interaction site random predictions . . . . . . . . . . . 60

4.2 Interaction site prediction errors (average DM) for PIPE-SItes compared to two other interaction site prediction methods. . . 60

5.1 Summary of data gathered for organisms processed by MP-PIPE2 70

5.2 Summary of predictive performance of MP-PIPE for organisms processed by MP-PIPE . . . . . . . . . . . . . 71

5.3 Comparison of LGTS-MS experiments in H. sapiens to PIPE predictions. .................. . . 79

5.4 PIPE predicted interactions between the highly sequence similar fibroblast growth factors (FGF) and their receptors (FGFRs). .

5.5 PIPE predicted interactions between the highly sequence similar cyclin-dependent kinases $(\mathrm{CDK})$ proteins and their regulators. .

5.6 High degree H. sapiens proteins and associated biological pro-

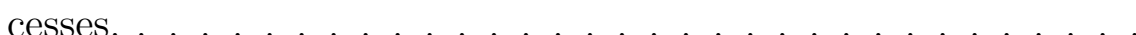

5.7 H. sapiens proteins with high betweenness centrality and associated biological processes.

6.1 A set of proteins expected not to interact with one another in Chlamydomonas, Gonium and Volvox .............

6.2 Summary of predicted PPI network statistics for Chlamydomonas, Gonium and Volvox as well as Chlorella and Coccomyxa. . . . . 103

6.3 Statistics on the size of the real (wild type) interactomes compared to the 100 simulated interactomes for each strain. . . . . 110

6.4 Comparison between the GO term enrichment for sets of conserved and highly evolving proteins determined with sequence based and PPI-based methods. . . . . . . . . . . . . . 
6.5 The intersection of enriched GO terms for proteins with lower than expected $\gamma$ and proteins with slowly evolving sequences. . 120

6.6 Enriched GO terms for proteins with higher than expected $\gamma$ not identified when analyzing highly evolving protein sequences. . . 121

6.7 Enriched GO terms for proteins with lower than expected $\gamma$ not identified when analyzing highly evolving protein sequences. . . 122

6.8 Enriched GO terms for proteins participating in conserved, significant PPIs. . . . . . . . . . . . . . . . . . . 124

6.9 Comparing the significant PPIs and their associated GO term enrichment between the four non-cerevisiae species. . . . . . . . 126

6.10 Unique enriched GO terms for the significant PPIs. . . . . . . . 126

6.11 Significant protein clusters and GO term enrichment analysis results found in each of the four non-cerevisaie species. . . . . . 127

6.12 Enriched GO terms for proteins making up a significant cluster in S. kudriavzevii. . . . . . . . . . . . . . . . . . . . . . 129

7.1 Set of $S$. cerevisiae proteins making up the candidate targets for InSiPS. . . . . . . . . . . . . . . . . . . . 155

7.2 InSiPS parameter testing results using the average aggregator in the fitness function. . . . . . . . . . . . . . 157

7.3 InSiPS parameter testing results using the maximum aggregator in the fitness function. . . . . . . . . . . . . . 158

7.4 Colony counts for each of the four S. cerevisiae strains after exposure to $65 \mathrm{ng} / \mathrm{mL}$ of cycloheximide. . . . . . . . . . 163

7.5 Cell colony counts for each of the four S. cerevisiae strains after exposure to 30 seconds of ultraviolet light. . . . . . . . . . 165 


\section{List of Figures}

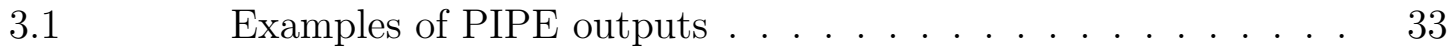

$3.2 \quad$ An overview of the PIPE Algorithm . . . . . . . . . . 34

$3.3 \quad$ Example of novel PPI detected by PIPE . . . . . . . . 35

3.4 Internal structure of a novel protein complex as predicted by PIPE . . . . . . . . . . . . . . 36

3.5 The effect of the modified median filter on PIPE outputs. . . . 41

$3.6 \quad$ MP-PIPE Benchmarking Test 1 Speedup Results . . . . . . 49

$3.7 \quad$ MP-PIPE Benchmarking Test 2 Speedup Results . . . . . 51

$3.8 \quad$ Analysis of novel C. elegans interacting protein pairs. . . . . 54

$3.9 \quad$ Analysis of novel H. sapiens interacting protein pairs. . . . . . 54

$4.1 \quad$ Example of the PIPE-Sites walk algorithm $\ldots \ldots \ldots 57$

$4.2 \quad$ Example of interaction sites predicted by PIPE-Sites $\ldots . .58$

4.3 Comparison between the calculation of the traditional PIPE score and the similarity-weighted PIPE score. . . . . . . . 66

5.1 ROC curves showing MP-PIPE predictive performance on a variety of organisms. . . . . . . . . . . . . . . . 72

5.2 ROC curves showing MP-PIPE predictive performance on crossspecies PPI prediction. . . . . . . . . . . . . . . 73

5.3 ROC curves showing MP-PIPE predictive performance using interaction data from a collection of organisms. . . . . . . . 75

5.4 Comparing the sensitivities and specificities achieved by PIPE and other methods $[96,176,259]$ as done in $[210] \ldots \ldots . .76$

5.5 The correlation between disease and hubs/high betweenness centrality in the predicted H. Sapiens interaction network. . . 84

5.6 Novel protein complexes extracted from the predicted H. sapiens interaction network. . . . . . . . . . . . 85

$5.7 \quad$ Schematic diagram of breast cancer pathway . . . . . . 87 
6.1 Pairwise comparison of degrees of homologous proteins between Chlamydomonas, Gonium and Volvox. . . . . . . . . . 100

6.2 Visualization of the changes in between PPI networks. . . . 102

6.3 Visual representation of the key network parameters with respect to modularity and integration of the predicted PPI networks for the studied organisms. . . . . . . . . . . . . . . 104

6.4 Cumulative histograms of key network parameters with respect to modularity and integration of the predicted PPI networks for the studied organisms. . . . . . . . . . . . . . 105

6.5 Phylogenetic tree for S. bayanus, S. kudriavzevii, S. mikatae, S. paradoxus and S. cerevisiae (reproduced from [249]) . . . . . 107

6.6 Histogram of $\gamma$ values for all 4,179 orthologous proteins across the 5 yeast strains. . . . . . . . . . . . . . . . 116

6.7 Comparing a protein's $\gamma$ value to its degree in the S. cerevisiae PPI network. . . . . . . . . . . . . . . 117

$6.8 \quad$ Comparing a protein's $\gamma$ value to its $\omega$ value. . . . . . . . 117

6.9 The $\gamma$ value of each of the 936 proteins identified as having rapidly changing interactions (red) with the respective range of $\gamma \mathrm{s}$ observed for that protein in the simulated phylogonies (black). 118

6.10 The $\gamma$ value of each of the 191 proteins identified as having rapidly evolving interactions (red) with the respective range of $\gamma \mathrm{s}$ observed for that protein in the simulated phylogonies (black). 118

6.11 Significant cluster found within the S. bayanus predicted PPI network. . . . . . . . . . . . . . . . . . . . 128

7.1 Graphical overview of the core InSiPS genetic algorithm. . . 138

$7.2 \quad$ Heat map of the InSiPS fitness function. . . . . . . . . 140

7.3 Run time results for InSiPS threads benchmarking test on the local lab cluster. . . . . . . . . . . . . . . . . . . . . 148

7.4 Speedup results for InSiPS threads benchmarking test on the local lab cluster. . . . . . . . . . . . . . . . . . . . . 148

7.5 Runtime results for InSiPS threads benchmarking test on the Scinet BGQ cluster. . . . . . . . . . . . . . . . . . . . 149 
7.6 Speedup results for InSiPS threads benchmarking test on the Scinet BGQ cluster. . . . . . . . . . . . . . . . . . . . . 149

7.7 Run time results for InSiPS worker process benchmarking test on the local lab cluster. . . . . . . . . . . . . . . . . 152

7.8 Speedup results for InSiPS worker process benchmarking test on the local lab cluster. . . . . . . . . . . . . . . . . . . 152

7.9 Runtime results for InSiPS worker process benchmarking test on the Scinet BGQ cluster. . . . . . . . . . . . . . . 153

7.10 Speedup results for InSiPS worker process benchmarking test on the Scinet BGQ cluster. . . . . . . . . . . . . . . 153

7.11 Fitness of the best generated sequence for each of the three experimental candidates over the generations of their respective InSiPS runs. . . . . . . . . . . . . . . . . 160

7.12 Average colony counts for each of the four S. cerevisiae strains after exposure to $65 \mathrm{ng} / \mathrm{mL}$ of cyclohexamide. . . . . . . . 163

7.13 Average colony counts for each of the four S. cerevisiae strains after exposure to 30 seconds of ultraviolet light. . . . . . . . 165

7.14 Spot test for the four S. cerevisiae strains grown for 48 hours after 30 seconds of exposure to ultraviolet light. . . . . . . 166 


\section{List of Abbreviations and Symbols Used}

ETI Evolutionary Transition in Individuality

FN False Negative

FP False Positive

GA Genetic Algorithm

GO Gene Ontology

HPCVL High Performance Computing Virtual Laboratory

InSiPS In-Silico Protein Synthesizer

MP-PIPE Massively Parallel Protein-protein Interaction Prediction Engine

PIPE Protein-protein Interaction Prediction Engine

PPI Protein-Protein Interaction

ROC Curve Receiver Operating Characteristic Curve

SVM Support Vector Machine

TN True Negative

TP True Positive 


\section{Chapter 1}

\section{Introduction}

The study of protein-protein interactions (PPIs) has seen a massive rise in interest over the past 15 years. The physical interaction between proteins determine the functions that the proteins have, allow for communication throughout the cell and act as the response to internal and external stimuli within the cell. These PPIs make up the overall internal machinery that drive a cell and are largely responsible for the overall internal state of a cell $[46,154]$. For this reason, the elucidation of individual PPIs, as well as entire PPI networks, has become of great interest to the scientific community. A greater understanding of the interactions proteins are involved in and the roles they play in the overall interaction network are expected to have a large impact with important practical applications, such as drug design. PPIs are central to a number of human diseases and are therefore a target for human therapeutics [12] but understanding the detailed workings of the cell at a molecular level is vital to successfully combat these diseases [242]. The elucidation of PPI networks will not only give us a clearer picture of how a cell operates, it is expected to accelerate the drug discovery process while at the same time reducing development costs [217].

Since the demand for experimentally confirmed PPIs is so high, wet-lab methods have been developed to determine these interactions. One of the more popular methods is yeast two-hybrid, introduced in 1989 [79]. This method is used to test if two given proteins (a bait protein $X$ and a prey protein $Y$ ) interact or not. This method works as follows. First, a transcriptional activator domain is fused to protein $Y$ and a DNA-binding domain is fused to protein $X$. If proteins $X$ and $Y$ come together to form an interaction, the two fused domains are also expected to come together. The assembly of these domains results in the transcription of a reporter gene. This reporter gene causes some kind of noticeable phenotype, such as the production of a coloured compound or an effect on the survival of the cell [46]. This system has been success-

fully used to perform large scale, high-throughput experiments on the Saccharomyces 
cerevisiae organism by Uetz et al [279] and Ito et. al [120], among others. Issues arose however when these resulting data sets were analyzed. The 1,519 interactions determined by Uetz, the 4,549 interactions determined by Ito (of which 841 were deemed high confidence) and a third set of 1,082 yeast-two hybrid determined PPIs (coming from 174 single publications) were extracted from the Munich Information Center for Protein Sequences (MIPS) database [180] and compared. These sets of results were found to have very little overlap, showing that the outcome of a given yeast-two hybrid experiment is very difficult to reproduce. Analysis on this comparison suggested false positive rates anywhere in the range of 44-91\% [189]. Another analysis of yeasttwo hybrid results in yeast, worm and fly experiments suggested false positive rates in the range of 9.9-17\% and false negative rates in the range of 28-51\% [112]. Tandem affinity purification coupled with mass spectroscopy, also referred to as TAP-tagging, is another popular method to experimentally determine PPIs. This method was introduced in 1999 [235] and works as follows. A TAP-tag is associated with a protein of interest and is placed on a column which will retain the tagged protein. This column is then introduced to a wide range of other proteins, allowing for the tagged proteins on the column to interact with them. After some time, the column is washed to remove any non-interacting proteins, leaving complexes of proteins interacting with the tagged protein on the column. These proteins are then identified with the use of mass spectrometry [46]. It should be noted that all of the proteins found are reported to interact with the tagged protein, yet these proteins may actually interact indirectly through a small number of intermediaries, producing potential false positives. The washing process performed near the end of the experiments could also remove transient interactors with the tagged protein, thus producing false negatives [46]. A study found that TAP-tagging produced an error rate of roughly $15 \%$ and also noted that this rate could be much higher in other experiments [86]. A variety of other experimental methods to determine PPIs exist (including high quality X-ray or nuclear magnetic resonance (NMR) crystallography [117], co-immunoprecipitation, protein microarrays, gene co-expression, synthetic lethality [261]) as well as methods to investigate specific PPI interaction sites (ie. Amide hydrogen/deuterium detected by 
matrix assisted laser desorption/ionization time-of-flight (MALDI-TOF) mass spectrometry (MS) [173]). These types of methods have been evolving and improving in recent years (eg. $[15,106,219])$, however they are all generally faced with the same issues. They suffer from high cost, time and expertise demands while also producing relatively high error rates [264].

In an attempt to overcome the drawbacks of wet-lab experimental validation computational approaches to the prediction of PPIs have become very popular over the past 15 years. These approaches attempt to take advantage of the wealth of biological data relating to proteins and their encoding genes to make their predictions. However, similar to the experimental methods, they all have their own shortcomings. Limited applicability, lack of underlying biological data, high computational costs or relatively high error rates afflict these computational approaches. PIPE (the Proteinprotein Interaction Prediction Engine), and its subsequent massively parallel version MP-PIPE, is a computational tool used to predict PPIs. Prior to the work presented in this thesis, MP-PIPE was able to perform proteome-wide, all-to-all predictions in a variety of organisms with an extremely high specificity of $99.95 \%$ in a relatively short amount of time, making it an attractive alternative to other PPI prediction methods.

The work presented in this thesis builds upon the strong base that PIPE and MP-PIPE provide. This thesis is organized as follows. In Chapter 2 a review of computational PPI prediction methods is done. Chapter 3 describes the history of PIPE and MP-PIPE up until novel work described in this thesis began. In Chapter 4, the various improvements and extensions to PIPE, developed by the author and others, will be described. Chapter 5 describes a variety of projects based off of the predicted PPI networks produced by PIPE. Chapter 6 discusses two projects which study the evolution of closely related organisms using their predicted PPI networks. Chapter 7 introduces InSIPS, a massively parallel tool used to create novel protein sequences with specific PPI-profiles.

\subsection{Summary of Contributions}

This thesis is the culmination of a variety of projects related to PPI prediction. The impact of the individual contributions made by these projects vary, but all are

presented here for completeness. In all cases, the following people collaborated or 
acted in supervisory roles to some degree: Dr. Sylvain Pitre, Prof. James R Green, Prof. Ashkan Golshani and Prof. Frank Dehne. Collaborators mentioned are (or were at the time) located at Carleton University, unless otherwise stated. The projects making up the contributions to this thesis fall under the following headings.

\section{- Further Development and Improvements of PIPE}

There are two projects in this category in which the author of this thesis directly participated.

- PIPE-Sites is a tool used to analyze PIPE output matrices and predict the sites of interaction on two proteins predicted to interact. PIPE-Sites was designed, implemented and tested by Adam Amos-Binks, described in [9] and Section 4.1.1. After this, the author of this thesis contributed to the further analysis of PIPE-Sites (specifically providing the InterPro analysis and participated in the discussion and writing of the manuscript) described in [10] Section 4.1 of this thesis. The analysis was then extended by the author of this thesis to provide the data which became the basis of Yuan Gui's master thesis described in [94] and Section 4.1.2. On top of the other collaborators mentioned, Catalin Patulea participated in the writing of the PIPE-sites publication [9].

- Catalin Patulea designed and implemented a new scoring function which outperformed the traditional PIPE scoring function as well as a relatively simple yet hugely effective performance improvement, described in Section 4.2 and [212]. These improvements were added to the PIPE2 implementation and could not be used by the current MP-PIPE implementation. To be able to fully utilize all advancements made on the PIPE project, the author of this thesis created a new version of PIPE, namely MP-PIPE2, which combined the improvements made by Patulea as well as the PIPESites algorithm (described in 4.3). MP-PIPE2 is the current version of PIPE used.

\section{- Applications of PIPE \& Analysis of Resulting Data}

Three projects which the author contributed to fall into this category of contributions.

- MP-PIPE was shown to be able to perform cross-species predictions in a 
variety of organisms (using known PPIs from one organism to predict those in another organism). It was then shown that MP-PIPE could use a collection of PPI data from a variety of organisms to successfully predict PPIs in another organism. Finally, extended analysis of a previous study where MP-PIPE was directly compared to three other PPI prediction methods was done. This work is described in Section 5.1 and in [225]. The author of this thesis was directly involved with all computational aspects of this work as well as participating in the writing of the published manuscript. Other collaborators for this project include biology graduate students (at the time) Mohsen Hooshyar, Bahram Samanfar and Matthew Jessulat.

- Novel predictions made in the Saccharomyces cerevisiae organism were used as the basis of a study to show previously uncharacterized proteins were involved in the cellular process of non-homologous end joining. This work is described in Section 5.2 and in [202]. The author collected updated S. cerevisiae data, performed the predictions, analyzed the results together with Sylvain Pitre and passed the data along to colleagues to perform further biological analysis and experiments. Major collaborators include biology graduate students (at the time) Katayoun Omidi, Mohsen Hooshyar, Matthew Jessulat, Bahram Samanfar, and Daniel Burnside. For a full list of collaborators, see [202].

- The predicted PPI network for H. sapiens, which was produced in [250, 253] and described in Section 3.3.3, was fully analyzed in a new study. This work consisted of many aspects, including the comparison of PIPE's results to new experimentally determined PPIs, showing PIPE's ability to distinguish the interaction partners of proteins with high sequence similarity but different function, an analysis of topological features of the PPI network, the identification of proteins involved in breast cancer and the identification of novel molecular markers for seasonal allergic rhinitis, among others. This study was the result of the collaboration of many people, but the author of this thesis provided the computational support to the biologists, the computational analysis and lead the writing of the manuscript. This work is described in Section 5.3 and [255]. Major collaborators for this 
project include Prof Alex Wong, and biology graduate students (at the time) Bahram Samanfar, Mohsen Hooshyar, Katayoun Omidi and Yuan Gui. For a full list of collaborators see [255].

- Using Predicted PPI Networks to study the Evolution of Closely Related Organisms

- PIPE predicted PPI networks were used to study the evolutionary differences between three closely related members of the volvocine family of green algae. Specifically, there exist members of this family in three distinct stages in the evolutionary transition from unicellularity to multicellularity. This transition was studied based on their predicted PPI networks with notable differences and trends being identified. This work is described in Section 6.2. A manuscript describing this work has been written and is being prepared for submission [254]. All computational work described as well as a significant portion of the writing of the manuscript was done by the author of this thesis. Biological motivation, analysis and conclusions courtesy of major collaborators Prof. Pierre Durand and Jonathan Featherston from the faculty of Health Sciences at the University of Witwatersrand, Johannesburg. For a full list of collaborators, see [254].

- In a similar study, PIPE predicted PPI networks were used to study five closely related yeast strains. In this study a novel null model used to analyze PPI networks was also introduced. This work shows the benefit in studying differences in PPI networks in addition to traditional comparative genomic methods. This work is described in Section 6.3. A manuscript describing this work has been written and is being prepared for submission [251]. All computational work described (except the generation of the simulated proteomes) as well as a significant portion of the writing of the manuscript was done by the author of this thesis. Collaborators for this project include Prof. Alex Wong, and biology graduate students Daniel Burnside and Houman Moteshareie.

\section{- Producing Novel Proteins with Specific Interaction Profiles}

The In-Silico Protein Synthesizer (InSiPS) is a massively parallel computational 
tool which can design novel protein sequences with specific PPI profiles. Specifically, it designs novel proteins which are predicted to interact with a target protein while avoiding interactions with a set of non-target proteins. Multiple wet-lab experiments were done to show that InSiPS designed sequences could be successfully used as inhibitory proteins. This work is described in Chapter 7 and in [252]. The design, implementation, testing and running of the algorithm as well as leading the writing of the manuscript was done by the author of this thesis. Collaborators for this project include Prof. Alex Wong, and biology graduate students Daniel Burnside and Houman Moteshareie. 


\section{Chapter 2}

\section{Protein-Protein Interaction Prediction Methods Review}

The problem of protein-protein interaction (PPI) prediction has been very well studied in the past 20 years. There exist a wide array of approaches, each utilizing a variety of different available data (surveyed in [84, 223, 264, 280] and more recently in [126, 163, 312]). In this chapter an overview of the approaches to PPI prediction methods will be given. Nearly all approaches fall into one of five categories. They include approaches based on genomic information, evolutionary relationships, 3D structure, PPI network structure, well known protein domains or machine learning techniques (including the subclass of approaches based solely on primary protein sequence). One should note, however, that some approaches combine data from two or more of these areas and could rightly be judged to fall within more than one of these categories. This chapter is meant to give the reader a general idea of the landscape of PPI prediction methods, pointing out highlights of each category as well as focusing on newer approaches. For each category, a sample of approaches will be examined, discussing the underlying ideas to these approaches and some general results will be presented, if available. It should be noted, however, that many of the approaches have wildly different testing schemes and testing data sets, which makes directly comparing approaches based on high level results problematic.

\subsection{Methods Based on Genomic Information}

The approaches classified as genomic methods predict PPIs using information taken from complete genome sequencing. The general idea behind these approaches is that by looking at the organization and relative ordering of genes within a certain organism, one can get an idea of which genes (and, by extension, the various proteins encoded by the protein-coding genes) are functionally related [84].

Tamames et. al showed that pairs of genes that were found to be conserved between two bacterial genomes implied, more often than not, that the proteins they 
encoded for interacted and that this could be used to successfully predict PPIs [269]. Dandekar et. al extended this idea by performing analysis on several genomes and found that, of the proteins encoded by conserved gene pairs, at least $75 \%$ of these pairs were shown to physically interact. Using this as a base, they went on to use these pairs of genes with conserved ordering to predict PPIs and protein function [53]. Overbeek et al. further extended this idea and took evidence regarding the conservation of gene ordering and clustering between genomes to build a system to predict functional coupling between genes. First, a notion of closeness was defined to determine whether or not any two genes were "close" to one another in a given genome. Secondly, the notion of bidirectional best hit (BBH) was defined such that, for two given genes $X$ and $Y$ from two genomes $G_{1}$ and $G_{2}$ respectively, $X$ and $Y$ form a BBH if and only if $X$ and $Y$ are relatively similar to one another and there is no other gene in $G_{2}$ more similar to $X$ than $Y$ and there is no other gene in $G_{1}$ more similar to $Y$ than $X$. Nearly 60,000 pairs of close, bidirectional best hits were found among 31 genomes. Functional relationships could be confirmed for $35 \%$ of these pairs, adding significant support to the hypothesis that conserved gene clusters retain functional relationships [203]. Snel et. al also used the conserved co-occurrence of gene pairs to not only make PPI predictions, but also predicted PPI networks. Through analysis of these networks they not only identified conserved PPIs but also identified functional protein clusters and sub-clusters which were also conserved [266].

Another popular approach under this classification uses the idea of gene fusion. The idea behind these approaches is that certain distinct proteins in a given organism exist as fused proteins in another organism and the underlying assumption is that if two proteins in a given organism are orthologous (equivalent) to a "fused" protein in another organism, then the two proteins are likely to interact. These fusion events are advantageous for single, united proteins as they are more efficient than two distinct proteins and the individual parts of the fused proteins will always be co-expressed as a single unit, which is not always a guarantee for two distinct proteins [199]. Enright et al. devised a scheme to search for these gene fusion events based on sequence comparison. Using this approach, they were able to identify a number of events of this type in a series of genomes. Within their results, they found a number of previously identified, well-known interacting protein pairs as well as a number of unconfirmed 
cases which could be considered interesting, testable predictions [69, 70]. Tsoka and Ouzounis followed up this work by showing that PPIs involving metabolic proteins are strongly linked to gene fusion events [275], adding confidence to this approach of PPI prediction methods. Iliopoulos et. al then went on to use gene fusion events to predict PPIs in Drosophila melanogaster. Through their analysis they identified a total of 18,654 gene fusion events. To filter this, they imposed a few restrictions (both genes and resulting proteins must share very high sequence similarity) and ended up with 220 high confidence predictions of functionally related proteins [119]. Marcotte et al. also proposed a very similar fusion-based approach which they named "domain fusion analysis". With their analysis they were able to predict protein pairs that have related biological functions and also predict potential PPIs [174]. Marcotte et al. went on to combine their approach with two other independent genomic approaches to create a large-scale predictor of protein function. The general approach was to take the union of the predictions of these approaches and then test the links created between given proteins. If protein $X$ is linked to a certain group of functionally related proteins, then the function of $X$ can be inferred based on the functionality of the group of related proteins. This idea can be tested when the function of $X$ is previously known. Applying this aggregated approach to yeast, it was found that of the roughly 2,500 proteins whose functions were previously uncategorized, $15 \%$ of them could be assigned a general function with a very high confidence and a total of $62 \%$ can be assigned a general function overall. It was thought that this approach should substantially increase the rate at which protein function is discovered [175].

\subsection{Methods Based on Evolutionary Relationships}

PPI prediction methods that fall within this category rely on the general hypothesis that the physical or functional relationships between proteins can be inferred based on the evolutionary relationships that the proteins have.

One such approach proposed by Pellegrini et al. uses phylogenetic profiling to characterize proteins as either likely or unlikely to interact. A phylogenetic profile of a protein simply represents which organisms, out of a given set of organisms, contain a homolog (a protein with a very similar amino acid sequence which has been evolutionarily conserved) to the protein in question. The profile for a given protein 
is simply a bit-string of length $n$ (where $n$ is the number of considered organisms) and each bit corresponds to an organism: if a given bit is 1 then the protein has a homolog in that organism, if it is 0 then it does not. The authors then went on to show that if two proteins have homologs in the same subset of organisms (had identical or very similar phylogenetic profiles) then they were likely to interact. They also showed supporting evidence to this hypothesis in that proteins that are thought not to interact do not have similar phylogenetic profiles. The authors noted however that not all interacting proteins have similar profiles, so this method is not perfect $[216]$.

Building off of the work done by Pellegrini et al., a more sophisticated way to measure the similarity between two phylogenetic profiles was created. To do this, the profiles were mapped into a high-dimensional vector space and then an algorithm was developed to calculate the inner product of two profiles represented in this space. This inner product was named the tree kernel, which belongs to a larger set of functions called kernels (ie. functions that calculate the inner product of two objects mapped into a vector space). With this tree kernel it is possible to define a distance between profiles in this vector space and, in this case, two profiles that are "near" each other are likely to have similar patterns of evolution. Support Vector Machines (SVM, a kernel-based supervised learning algorithm) were then used on phylogenetic sequences using the tree kernel as their kernel. It was shown that SVMs using the tree kernel performed much better on average than SVMs using a naive kernel at predicting PPIs $[282]$.

The use of the binary phylogenetic profiles was extended in another work to compare the phylogenetic trees of proteins and use these to predict how likely the given proteins were to interact. In comparison to a phylogenetic profile, a phylogenetic tree shows the evolutionary relationship between a group of organisms that have a common ancestor. Each node in the tree represents an ancestor to all of that node's children, and the further away two nodes are from one another in the tree, the less similar the organisms are. Goh et. at showed that proteins that evolved together (ie. they were carried from organism to organism in the phylogenetic tree) were likely to interact and provided a way to measure this phenomenon [87]. Pazos and Valencia extended this idea by devising a method in which the phylogenetic trees of 
proteins could be compared and then a prediction on whether the proteins interact or not could be made. This approach proved to be promising when compared to other evolutionary and genomic approaches [213]. Pazos and Valencia also introduced a method which searches for distinctive mutations based on the hypothesis that interacting proteins can be predicted based on co-adaptation (the presence of correlated mutations which compensate for one another in a pair of proteins). They applied their method, named In-Silico Two-Hybrid (i2h), to a number of relatively small test sets and found that true interacting proteins scored higher than other protein pairs expected not to interact [214].

Another approach that falls under this category is approaches which search for "interologs". An interolog refers to a pair of proteins which have homologs in another organism that are known to interact. Walhout et. al introduced the concept of interologs and showed that they could be used to infer both known and novel PPIs in C. elegans from other model organisms [285]. Yu et al. extended this idea and showed that interologs could be verified as PPIs when transferred between a number of organisms. They then also map interologs from well-studied model organisms to those with limited known information, representing large-scale, novel PPI predictions [309]. This general idea was extended by Sharan et al. who took interaction data from Caenorhabditis elegans, Drosophila melanogaster and Saccharomyces cerevisiae and aligned their respective PPI networks by aligning sequence similar proteins from the different organisms. In doing this, they could see interactions that were conserved between the organisms as well as use data from two of the organisms to predict interactions and protein function in third organism. As a result they predicted 2,609 novel protein interactions. They then went on to test 60 of these interactions through yeast-two hybrid experiments, confirming nearly half of them [257]. Lee et al. further extended this idea by identifying interologs between 18 eukaryotic species. In this extended work they were able, for example, to make more than 32,000 predictions in human, 3,859 of which have been confirmed experimentally, as well as 3,090 interspecies interactions between human and the malaria causing Plasmodium falciparum [152]. 


\subsection{Methods Based on 3D Protein Structure}

Approaches to PPI prediction that fall within this category rely heavily on the 3D structure of proteins that are known to interact. The underlying hypothesis that these methods have in common is that structural information of interacting proteins can be extracted and applied to similar proteins which then can predict further interactions [265]. One should note that these approaches are only applicable to those proteins for which 3D models have been previously determined or for those proteins for which a model can be inferred from similar proteins with known 3D structures. It is expected that as these structures become more readily available, these approaches will increase in popularity [14].

A method outlined by Aloy and Russell works along these lines. Their method predicts how well two potentially interacting proteins fit together based on a database of known 3D structures and then infers how likely the proteins are to interact based on the molecular details of the interaction sites, compared to those in the database. Their algorithm works in the following way. First, the protein sequences are searched for specific, predetermined domains (shorter protein segments that have a known function). They then check their database of known interacting domains (for which the 3D structures are known) for any instances of the domains found within the query proteins. If each protein contains a domain that matches a pair of interacting domains in this database, then the proteins are aligned on these segments. Using this alignment, a protein interaction prediction can be made. To do this, they check whether the query proteins contain the same residues that make the atomic contacts of the interacting proteins in the database. From this they can estimate a statistical significance for the potential interaction [7]. This method was subsequently made publicly available through a web portal called InterPreTS (protein Interaction Prediction through Tertiary Structure) [8].

In this same vein, Stein et al. created 3did, a database containing a collection of domain-domain interactions for which the 3D structures are known in high resolution. These 3D structures are exploited to provide a detailed analysis for interactions between proteins. This database was built on protein domains rather than full protein sequences and new 3D structures are incorporated regularly. The main way to retrieve information from this database is to present it with a particular query domain. 
3did will then search for this domain and present all other domains that it interacts with and this will then be displayed as an interaction network. From here, the user can select a particular interaction to receive more details. Finally, the user can also get a $3 \mathrm{D}$ visualization of the given interaction if they so desire. The database can also be queried with a protein sequence itself. From this sequence, 3did will identify specific domains and then display interaction links from all of these domains to domains stored in the database [268].

Some methods rely on a technique called threading to build approximate 3D structures. This technique attempts to identify recognizable motifs or sequence-signatures which can be assigned predetermined 3D folds. These combinations of predetermined folds make up a model of the protein in question in three-dimensional space. These models are then evaluated based on a set of empirical potentials inferred from known protein structures [129]. One of the earliest and most popular threading approaches is a method called THREADER [128]. Lu et. al presented MULTIPROSPECTOR, a tool to predict PPIs based on 3D structure. Their method relies on a threading method called PROSPECTOR [263]. The method first produces 3D structures using PROSPECTOR and then, using statistical interfacial pair potentials, the interaction energy between the query proteins is computed and, if high enough, the proteins are predicted to interact. Lu et. al went on to apply their method to a small number of test cases [167].

Espadaler et al. developed a two-pronged approach to protein-protein interaction prediction. First, they took a set of 3D structures for interacting proteins and extracted the interface sequences of the proteins along the sections where they interact. With these interface sequences, they searched through a set of proteins, looking for proteins which contained these sequences. If two proteins could be found that each contained a segment that is very similar to an interface on two interacting proteins, then these proteins were considered a possible interacting pair. The second part of their approach involved searching for homologs of the proteins known to interact. In this part, if two proteins are homologs to two proteins that are known to interact, then they are considered a potential interacting pair. After this was done, the intersection of the two sets of predicted interacting pairs were taken. This then meant that each pair in this set of pairs of proteins had the following properties: 
- the pair of proteins each contain a segment that is very similar to the interaction interface of a pair of proteins that are known to interact

- the pair of proteins have a similar structure to a pair of proteins that are known to interact

This set of potentially interacting protein pairs was quite large. A portion of this set could be confirmed or at least supported with experimental evidence, leaving a large portion of this set as newly reported, potentially interacting pairs [72].

Another approach in this category is PRISM (protein interactions by structural matching), developed by Ogmen et al.. Similar to other methods in this category, they relied on the hypothesis that if two proteins contain particular regions that are similar to regions of proteins that are known to interact, then it is thought that the initial proteins possibly interact through these regions. Their main algorithm takes two sets as input: a "template" data set (a data set for which the interactions and 3D structures are known) and a "target" data set of which one would like to map the potential interactions. Then, the target proteins are compared to the template proteins in order to find template proteins that are "structurally similar" to the target proteins. This comparison leads to a similarity score being generated. If two proteins are "similar" enough to a pair of interacting proteins in the template set, then they are predicted to interact (the similarity score contributes to the overall prediction score). The web interface of PRISM also allows the user to put in their own query proteins that were not included in their target set to predict possible interaction partners [200]. The PRISM web portal has recently been updated and now has more features including visual structure models for queries, the ability to browse accumulated queries in their database as well as the ability to browse protein interaction interface templates used for prediction [16].

Hue et. al combined the use of 3D structures and the statistical pattern recognition power of a support vector machines (SVM). To do this, they collected a large number of experimentally validated PPIs, constructed a set of proteins expected not to interact as well as collected a large number of 3D protein models. They then used BLAST to compare the proteins making up the known interactions, as well as the pairs of proteins expected not to interact, to the sequences of the proteins making up their set of 3D models. Now, for each positive and negative pair, they would 
have a corresponding 3D model from relatively similar proteins. Using these positive and negative sets they could then train their SVM. They tested their method with a variety of kernel functions and also produced several thousand novel PPI predictions [114].

Struct2Net works in a similar way to the methods described above. The algorithm runs in two stages. In the first stage, the query proteins are compared to a library of protein structures using BLAST and the closest matching 3D models are chosen. In the second stage Struct2Net then evaluates how well the structures fit together and, based on this, predicts whether the proteins interact or not. Struct2Net is made publicly available though a web portal where a large number of predictions for model organisms are precomputed for quick look-ups. The portal also allows users to enter custom queries with proteins from other organisms for Struct2Net to make predictions on $[262]$.

Wass et. al presented a method which relies on a protein docking algorithm. These algorithms (recently surveyed in [113]) were typically used on protein pairs that are known to interact to build a model of the protein complex they form and potentially predict the specific interaction sites between the two proteins. They went on to show that approaching the problem of PPI prediction in this manner may not necessarily result in accurate 3D models of the interacting proteins as a pair but that the resulting output can be used to predict if the query proteins interact or not [288].

More recently, Li et. al presented a method which utilizes predicted sites of interaction to improve their predictions. The predicted interaction site is used as a constraint when trying to dock two query proteins together. Once a 3D model of the proteins is created, the algorithm is run again with updated interaction site predictions based on the previous run. They showed that their method outperformed other methods on a set of benchmark experiments and that the incorporation of predicted interaction sites consistently improved the prediction accuracy as compared with docking without using interaction site prediction [156].

Zhang et al. combined the use of 3D structures with a wide range of other, nonstructural information (co-expression data, functional similarity, evolutionary similarity, etc) to build the PPI prediction method PrePPI. PrePPI outperformed other methods based solely 3D structure data and, at a reported false positive rate of $0.1 \%$, 
predicted 31,402 and 317,813 PPIs in yeast and human, respectively [316].

Matsuzaki et. al presented MEGADOCK, a massively parallel tool based on rigid docking for PPI prediction. Their testing focused more on computational performance than on biological accuracy and was based on 176 PPIs with known 3D structure. They do show that their current method outperforms previous versions and that the quality of the prediction (ie. the distance from the known 3D structure of the interacting proteins) increases dramatically if the algorithm is run a number of times. They also reported a number of novel predictions using their method [177].

Ohue et. al combined the use of template matching (similar to PRISM, MULTISPECTOR, etc) with a docking method to come up with a consensus approach. They went on to show that their consensus method generally outperforms methods that rely solely on template matching, specifically comparing themselves against PRISM and MEGADOCK for a set of test problems [201].

The Critical Assessment of PRedicted Interactions (CAPRI) experiment is a community-wide effort to evaluate the ability of protein docking methods to predict how proteins interact in three dimensional space based on their 3D structure $[122,123]$. This competition has been running for nearly 15 years now. At the most recent evaluation, the results suggested a sustained and robust performance by all participating methods, with some new groups reaching higher than $80 \%$ success rate for the first time. There was also a noteable performance improvement for automatic docking methods, those algorithms which need no human intervention to make highquality 3D PPI predictions [26]. It is hoped that this performance can continue to improve as novel 3D protein complexes are released via the CAPRI competition.

\subsection{Methods Based on Network Structure}

Approaches that fall into this class of PPI prediction methods are those that use either the local or global graph structure of the PPI network to predict PPIs or eliminate potential false positives present in the PPI network made by other experimental or computational methods.

Saito et. al introduced the "interaction generality measure" which can be used to assess the reliability of a given PPI. For a given interaction, this measure essentially assesses the surrounding network and identifies how many interacting partners each 
protein in the PPI has. If their interaction partners are made up of isolated proteins who do not interact with any other proteins, the pair will receive a relatively high score. On the other hand, if their partners are well integrated into a relatively dense part of the network, the pair would receive a low score. They went on to show that low scoring yeast pairs represented reliable interactions and high scoring pairs are most likely false positives $[245,246]$. Pei and Zhang extended this work by introducing a topological measurement used to integrate different PPI data sets. Their measure would identify reliable PPIs based on the similarity of their PPI profiles in the given data sets while also exploiting the small world properties of known PPI networks [215]. Chen et. al introduced a similar method called IRAP (interaction reliability by alternative path) which aims to remove false positives as well as false negatives from experimentally determined PPI networks. Their method assigns interaction probabilities to pairs of proteins based on the alternate routes between the two proteins in the PPI network. The stronger the connection between the proteins via alternate routes, the higher the probability that they actually interact. Conversely, a potential false positive would have little connection between the proteins outside of their direct connection. They went on to show that their method leads to increasingly better interactomes in terms of functional homogeneity of the protein partners in the interactions as well as PPI networks that contain lower fractions of false positives and negatives [40]. You et. al expanded on this idea and transformed the original input graph such that it could be projected into a low dimensional metric space, based solely on network structure. By doing this, it recasts the problem of predicting PPIs to finding similar points in this space. They introduced a reliability index which represents the likelihood that two proteins interact based on their projected location. They found that many of the high scoring protein pairs that they predict to interact are known to be functionally related or are found in the same cellular location. They also reported that their method can reach a sensitivity and specificity of $93 \%$ and $92 \%$, respectively, on their test sets containing the union of a few different yeast PPI networks [301].

Wuchty introduced a method combining both domain information (discussed in the next section) and local network structure. In this method, protein pairs containing pairs of domains expected to be involved in PPI behaviour are taken with 
their local graph contexts and their clustering coefficients (a measure of the degree to which nodes in a graph tend to cluster together) are calculated. Based on these results, combined also with gene expression data, the potential interaction is evaluated. Using their method they identified 1,428 potential PPIs between human proteins and proteins from the malaria-causing parasite Plasmodium falciparum [290]. Dyer et. al presented a very similar approach to predict host-pathogen PPIs. First they use protein-domain profiles to come up with an initial list of potential host-pathogen PPIs. They then exploit the network topology of the host network to identify host proteins predicted to interact with the same pathogen protein. The distribution of the distances between the host proteins in this case are calculated and this is done over all predicted host-pathogen PPIs. The same is done in the pathogen network. This data, then coupled with gene expression and Gene Ontology functional information, is used to filter the original list of potential PPIs. Using this method, they identified 516 potential human-P. falciparum interactions [66]. This idea was extended by Chen et. al who also incorporated domain information into a network of known yeast PPIs and built a function to score the interactions within the network. The major difference between their method and the previous ones is that they attempt to predict PPIs between two partners who have a common third party interactor. This leads to the consideration of protein triples instead of simply protein pairs. Using a smaller subset of their data as a gold set, they achieved a sensitivity and specificity of $83 \%$ $[42]$.

More recently, Wang et al. introduced a network-based method based on sparse matrix completion techniques. When given a set of known PPIs, these can naturally be represented as a graph. This graph can then be represented as an adjacency matrix, where the interactions are represented as a 1 in the matrix index positions corresponding to the proteins interacting. The task of predicting PPIs can then be recast as the problem of adding to this adjacency matrix. Based on the fact that the adjacency matrices for PPI networks are known to be low-rank and that accurate, if not exact, matrix reconstruction or recovery can be done on partial matrices known to have low rank, Wang et al. employ matrix completion methods to identify potential PPIs. In doing this, scores are assigned to all of the cells in the matrix that refer to protein pairs not known to interact. Then, using a cutoff point, any pair above the 
cutoff is a predicted PPI. In a series of 20 -fold cross-validation tests they were able to achieve precisions and recalls as high as $40 \%$ and $60 \%$, respectively, in some tests [286]. Another method was recently presented by Wang and Tang. Their method first searched the PPI network (made up of both known and predicted interactions) for cliques. Cliques that were above a given size and don't contain too many predicted PPIs were considered for further investigation. Using the suitable cliques found, interactions are predicted for those proteins that can be added to a clique of size $k$ by inserting a single edge to produce a clique of size $k+1$. Once all of these predictions have been made, Gene Ontology (GO) data is used to filter the predictions. Two rules based on the GO data are used:

1. Two proteins in a predicted PPI need to have a common cellular component.

2. Two proteins in a predicted PPI need to have a common molecular function.

Those PPIs which pass rule 1 are placed into a low confidence set of predictions (ALL) and those PPIs which passed both rules are places in to a high confidence set (CORE). On a set of 4,997 proteins and 23,233 interactions extracted from the Database of Interacting Proteins (DIP) [247], 352 CORE and 874 ALL PPIs were predicted. $92 \%$ and $89 \%$ of these predicted interactions could be verified in BioGRID [37], respectively [298]. Lou et al. also recently proposed a similar method which takes partial PPI networks determined via high-throughput wet-lab experiments and aims to eliminate false-positives from this data as well as predicting new interactions using it. Their method is based on a Collaborative Filtering framework, a technique used in e-commerce for personal recommendation, which aims to identify connections between elements based on incomplete data. Their method first converts the PPI network to an adjacency matrix and then considers each row as a feature vector describing the respective protein. They then present a function which compares these vectors, where high scoring vector pairs are considered similar and therefore are likely to interact and low scoring vector pairs likely do not interact (or represent false positives in the original data). They compared their method against three other methods (including the method described by Saito et. al above) and showed that their predictions outperformed the others with respect to Gene Ontology analysis [169]. 


\subsection{Methods Based on Protein Domains}

Approaches that fall into this class are approaches that try to identify previously defined protein domains (protein segments which have been previously determined to have some noteworthy properties) that participate in protein interaction. The identification of these domains found in interacting proteins could then be used to predict interactions in other protein pairs. Some of these methods focus in on a smaller class of domains known specifically to be involved with PPIs called peptide recognition modules (PRCs) [233].

Sprinzak et al. developed an approach that looks to identify pairs of sequencesignatures from a database of experimentally determined interacting proteins and then use the pairs of sequence-signatures to predict PPIs. First a large database of yeast protein pairs known to interact was collected. Each protein in this database was then assigned signatures (domains) that are defined in another yeast database known as InterPro [115, 184]. The signatures in this database are in the form of regular expressions, profiles, fingerprints and hidden Markov models, all compiled from various other databases. They then counted the combination of these signatures in the database of known interacting protein pairs to identify sequence-signature pairs that appeared in many pairs of interacting proteins (where one protein contained one sequence and the other protein contained the other sequence). The authors then concentrated on the sequence-signatures that occurred more often than expected at random to be sure that these signatures were relevant. Using leave-one-out crossvalidation on subsets of known interacting protein pairs, they were able to reach sensitivities as high as 97\%, however the authors note that these test groups were quite small. The authors also mention the fact that they still must rely upon the InterPro database to define the sequence-signatures, and therefore are limited by that [267]. Chen et. al then built off of this idea to build the "association probabilistic method" which works in a similar manner by predicting if protein pairs interact based on the domains they contain and the number of times they were observed in their training data. Using this method they showed that they outperformed many other methods available at the time [41].

Another approach similar to this was developed by Kim et al. In their approach, they took advantage of two separate databases. The first was a database containing 
experimentally determined interacting protein pairs called the Database of Interacting Proteins (DIP) [247] and the second database was the InterPro database discussed in the previous example. Their stated assumption was that if the domain pair $(a, b)$ is found in the interacting protein pair $(A, B)$ then it is considered a potentially interacting domain pair, and the more often this domain pair was found within interacting proteins then the more likely it is a truly interacting domain pair. Their method was defined as follows. First, 10,435 interacting protein pairs from DIP were extracted. Within these interacting pairs, the 5,849 unique protein sequences were scanned for defined domains from InterPro. They then went on to build a potentially interacting domains (PID) matrix, where every combination of potentially interacting domains was considered and a score representing their prevalence was stored. You could then use this matrix to do PPI predictions for two query proteins by simply searching the proteins for domains, and then seeing how likely these domains are to interact by checking their PID score. Using cross-validation it was found that the average sensitivity was $50.1 \%$ of the domain containing pairs of proteins. This dropped to $27 \%$, however, when all proteins in DIP, not just proteins containing domains, were considered. On the other hand, their test results showed a specificity of $98.4 \%$, which is quite promising [134].

Another domain based approach was presented by Deng et al. Their approach is similar to the previous two in that they relied on a set of known interacting protein pairs and a set of predefined protein domains, however they used the domains defined in the PFAM (Protein Family) database [80]. Their algorithmic approach was also different. They presented two separate approaches, the better of the two relied heavily on probability theory. The approach was to utilize maximum likelihood estimation to infer interacting domains from the known interacting pairs and then apply this to a set of proteins in order to predict some novel PPIs. To test their theory, they applied it to a set of proteins in which the interactions are already known and they found that their prediction rate is about 100 times better than that of random assignment $[59]$.

$\mathrm{Ng}$ et. al developed a method called InterDom which uses multiple methods and available data sets (including known domain-domain interactions, PPIs, protein complexes as well as other scientific literature) to predict PPIs. By comparing the 
observed frequency of domains co-occurring in the collected data sets (compared to what would be expected to be seen at random) a confidence score can be assigned to a given potential domain-domain interaction. Using this confidence score, false positives can be eliminated. Using their method they identified 5,511 domain-domain interactions through an exhaustive search through the union of all data sets used. Based on their confidence scores however, 3,308 of these are potentially false positives, leaving the rest as high confidence, predicted domain-domain interactions [195, 196].

A more complex system titled PreSPI was introduced by Han et al. which tried to overcome the earlier approaches' tendencies to assume that PPIs were mediated through a single pair of domains. With this approach they wanted to examine more possibilities by considering the mediating factor of a PPI to be groups of domains instead of just a single pair. In practice, this means that every subset of domains within a pair of interacting proteins are examined and are considered potentially responsible for the interaction. So if protein $A$ contains $n$ domains and protein $B$ contains $m$ domains then instead of simply considering the $m \times n$ potentially interacting domain pairs, the $\left(2^{n}-1\right) \times\left(2^{m}-1\right)$ different potentially interacting domain combination pairs from both proteins need to be considered. The authors then went on to build a matrix to be used for interaction prediction, similar to the matrix used by Kim et al. However, this matrix was an $n \times n$ matrix, where $n$ is the total number of domain combination groups identified in the training set of interacting protein pairs. This then allowed for a cell for each domain combination pair which contained a value representative of the pair's prevalence in interacting proteins. Using matrices built like this, a prediction on two query proteins could be made. Using this method they could also rank the quality of their predictions by assigning their prediction as a probability. In testing their approach, they were able to reach a sensitivity of $77 \%$ and a specificity of $95 \%$. It was also noted that, in general, their ranking method seemed to work well since a large portion of the true positives that were predicted to interact had a high interacting probability and a large portion of the true negatives that were predicted not to interact had a low interaction probability. The authors note however that this ranking method should be applied with caution, especially when an interaction probability between 0.4 and 0.6 is received, because this is where some problems could be found [99, 100, 101]. 
In terms of more recent domain based methods, Jang et. al presented a method that not only predicts PPIs based on domain interactions, but also supplied the information on which pair of domains or set of domain combinations are most likely responsible for the interaction between the proteins predicted to interact. To do this they took a large set of interacting protein pairs, identified the domains within the proteins and then extracted every possible combination of domain pairs from each PPI. Using this data they built a system that, given two query proteins, extracts the domains, evaluates how likely they are to interact given all possible domain combinations and then reports the combination of domains responsible if the proteins are predicted to interact. Through a series of tests, they observed sensitivities and specificities as high as $94 \%$ and $84 \%$, respectively [121]. Priya et. al also presented a method which uses high-throughput PPI data in which every protein has been evaluated against all other proteins, giving not only a large set of PPIs but also a large set of proteins known not to interact. Using this data set they then inferred both positive and negative domain-domain associations with which, given a new query protein pair, could be used to make predictions. Using a test set, their method had a sensitivity of $68.1 \%$ and a specificity of $65.3 \%$ [22].

\subsection{Methods Based on Machine Learning Techniques}

The past decade has seen a dramatic increase in the number of approaches using supervised machine learning techniques to predict PPIs. Some of these methods use a relatively small amount of data while others combine any and all available biological data in order to better their predictive power. This data includes amino acid sequences, domain information, genomic data, evolutionary data, Gene Ontology data, 3D structure, both local and global PPI network features, detailed physical attributes of the proteins in question, among other information. Generally speaking, these approaches use their base data to build a set of features which characterize protein pairs that interact and those that do not. This data is encoded into feature vectors and then, using a set of both positive and negative examples, the classifiers are trained. Once the training is completed, the machine learning algorithm has hopefully extracted the necessary features from the examples provided to then be able to make successful predictions on pairs it has not seen before [163]. The main 
notable difference between these methods is the choice of features and qualifiers [126].

One of the most popular machine learning techniques used for PPI prediction is the support vector machine (SVM) $[18,24,25,34,38,60,89,95,96,98,114,164,160$, 165, 176, 234, 259, 260, 267, 287, 291, 304, 305, 313, 317, 318, 319] (as well as other approaches using related techniques of support vector regression (SVR) and relevance vector machine (RVM) [131] and relaxed variable kernel density estimator (RVKDM) [35]). An SVM is a binary classifier (here the classes would be interacting or noninteracting) which builds an internal model, based on the provided training data. This model is built by plotting the training data as points in a plane, hopefully with a clear separation. Once trained and presented with new a new query, the SVM plots this as a point on the plane and determines which class it belongs to based on how close it is to either cluster. Although these methods can produce impressive sensitivities and specificities, they can often times be highly biased by the training data, also known as over-fitting. This leads to the situation where the SVM can successfully classify the training/testing data but suffer relatively low novel PPI prediction rates [126]. A study by $\mathrm{Yu}$ et. al showed that the ratio of positive to negative training examples a SVM is given has a drastic effect on the overall predictive accuracy of the SVM, seeing a drop in sensitivity of over $50 \%$ in some cases when changing the positive:negative ration from 1:1 to 1:15 [305]. This is indeed worrying given that actual PPI networks are expected to be extremely sparse and given the range of estimates reported in previous studies (1:100 and higher) [210, 305].

Another technique is the use of artificial neural networks $[75,76,170]$. A neural network was originally designed to mimic the activity of the human brain. Typically these networks have multiple layers of nodes (input, intermediate and output layers), with each layer being fully connected to the previous and following layer through weighted edges. Each node has an internal activation function which send outputs depending on what it receives as input. These weights are adjusted during training to be able to classify protein pairs as interacting or not [312].

A Naïve Bayes classifier is a simple probabilistic classifier based on Bayes' theorem. As a classifier it is popular due to its relative simplicity, due in large part to the fact that it assumes all of the random variables with respect to protein features are independent. However, in spite of this simplicity, a number of methods incorporating 
a Naïve Bayes classifier have recently emerged [23, 28, 110, 124, 159, 168, 191]. An averaged one-dependence estimator is a related classifier that was designed to try to overcome the variable independence problem associated with traditional Bayes classifiers. A PPI prediction method has recently been introduced using this type of classifier [190].

A decision tree is a tree used to arrive at a decision through a series of conditions. In this case, one would build a decision tree for a given protein or protein pair feature. The nodes internal to the tree would test this feature in some way and the path you would follow would be based on the outcome of this test. The leaf nodes of the tree would assign a class to the given problem. When one wants to test against multiple features, one would build a decision tree for each feature and then take the consensus. This technique is referred to as a random forest. Both decision trees [315] and random forests [45, 44, 171, 185, 206, 229, 237, 300] have been applied in a number of PPI prediction methods, although they are prone to over-fitting like the SVM-based approaches [312].

Although the above mentioned techniques seem to be the most popular, other machine learning techniques are also used in PPI prediction (such as K-nearest neighbours classifiers [93, 192], extreme learning machines [302, 303] and data mining algorithms adopted from transactional database analysis [111]). On top of this, consensus approaches (built using multiple other machine learning techniques), approaches which test a variety of machine learning techniques before choosing one or approaches that use a series of machine learning techniques (ie. the output of one is fed as input to another) have also been introduced [5, 50, 158, 209, 210, 228, 230, 240, 244, 291, 320]. Recently Saha et. al directly compared the use of SVMs, decision trees, random forests, naïve Bayes and artificial neural network classifiers for PPI prediction and found that SVM consistently outperformed the rest, which is logical based on their popularity in PPI prediction methods [243]. It should be noted, however, that $\mathrm{Yu}$ et. al did a study on the predictive performance of PPI prediction techniques which employed simple, sequence-based kernels (specifically evaluating methods [18, 259]) and found that the high accuracies reported for these methods are an artifact of the data sets used to train and test the methods. This is due to the fact that they were inherently biased because of the how the data sets were designed and it is likely that 
this is the same for similar methods [310].

\subsubsection{Subclass of Approaches Based on Primary Protein Structure} Alone

Some of the methods referenced in this section thus far have used some combination of data (protein sequence, domain information, genomic data, evolutionary data, among others) to train their classifiers and make PPI predictions, however others fall into an important subclass of these methods which are based on primary protein structure alone. In these approaches, the general hypothesis is that the interactions between proteins are mediated through short polypeptide sequences, or simply a sequence of amino acids, that define the primary structure of the protein. It is thought that these sequences can be extracted from sets of interacting protein pairs and then applied to proteins in order to predict possible interactions. These methods are generally the most applicable as they usually only require the protein sequences of the known interacting proteins as well as the query proteins and a set of known interactions, which is considerably less than the amount of data needed for other methods. Another added benefit is that the sequence information is among the most basic of protein data, meaning it is readily available thus making the application of these approaches much easier.

An example of one such approach was introduced by Bock et al. In their approach, they would take two similar organisms, one of which a set of interacting protein pairs is known and the other of which nothing is known. The main idea behind their work is that since the organisms are similar, then they will contain very similar proteins (homologs) and the proteins in the second organism will most likely function in a very similar manner. Based on this, PPIs can be predicted in the second organism based on the proteins that interact in the first organism. To do this, SVM learning algorithms were used to "learn" the PPIs in the first organism. After testing and tuning the parameters based on this organism, the proteins from the second organism could be fed into the SVM. The SVM would then construct an interaction network on these proteins, based on what it had learned from the interaction in the first organism. The authors noted that, from here, it would be possible to experimentally validate the results and then, if this interaction network is deemed to be relatively 
correct, it could be used to predict interactions between proteins in organisms similar to it. Not only was the precision and sensitivity pretty impressive for their tests $(80 \%$ and $69 \%$ respectively), the more impressive results were more general. It was shown that the interaction networks produced using this method were strikingly similar to experimentally derived networks in terms of average connectivity and cluster size distribution within the interaction networks. These facts add more biological credibility to their results [25].

Roy et al. have more recently developed another approach that use basic amino acid composition (AAC) features to predict PPIs. These features are strictly based on normalized counts of single or pairs of amino acids within the query proteins and proteins for which interactions have been previously determined. Through the use of various different classifiers, they found that their interactions predicted in yeast were of comparable accuracy to those predicted using previously defined protein domains. An interesting aside that supports their hypothesis was that AAC features that were deemed to be important to PPIs were statistically over-represented in domains known to be involved with PPIs. This suggests that AAC alone can capture a significant amount of information needed to predict interactions [240]. 


\section{Chapter 3}

\section{History of PIPE and MP-PIPE}

Although the methods described in the previous chapter have provided a tremendous amount of insight into PPIs and PPI prediction, they still all have their own limitations. These limitations come in many forms. Firstly, the amount of data needed for these methods to make their predictions can make it hard or impossible to apply a given method to a wide range of protein pairs. Secondly, the relatively low specificities (high false-positive rate) make it impossible to do massive, high-throughput predictions as the false predictions would significantly overwhelm any true PPIs detected. Lastly, the running time of the previously described methods would make it impossible to completely scan all possible protein pairs within a given organism. For a method to be able to do these proteome-wide, all-to-all predictions these three issues would need to be addressed. In this chapter a method that successfully did this will be described.

The Protein-protein Interaction Prediction Engine (PIPE, PIPE2), and its subsequent Massively Parallel versions (MP-PIPE, MP-PIPE2), is a computational tool used to predict protein-protein interactions. The basic PIPE algorithm (and all subsequent versions) rely solely on the amino acid sequences of the query proteins, a list of known interacting protein pairs, as well as the amino acid sequences for these known interacting pairs, to make its predictions. The main hypothesis that the en-

gine is built upon is that PPIs are mediated by short sequences of amino acids which are common across many protein interaction sites within a given organism. The fact that PIPE relies solely on the amino acid sequences (query pairs as well as known interacting protein pairs), gives it a distinct advantage over other methods which need significantly more information about their query pairs, such as 3D structure or known domains. This is advantageous since PIPE can be applied to any pair of proteins given their primary sequences and a suitable number of known interactions from either the same host organism or from a closely related organism. This chapter will give the 
history of PIPE from the beginning to the point in which the new work described in this thesis begins, and is laid out as follows. In Section 3.1 the main PIPE algorithm will be detailed as well as preliminary results achieved with this version. Section 3.2 will detail significant performance improvements made upon the first version of PIPE and the results obtained using these. Section 3.3 describes performance issues that still existed in PIPE2 and how they were addressed in the massively parallel version of PIPE, MP-PIPE.

\subsection{PIPE}

The Protein-protein Interaction Prediction Engine is a computational tool used to predict whether two proteins interact or not. This section describes how the sequential PIPE algorithm makes its predictions and summarizes the results from the first PIPE publication [224].

\subsubsection{The Main PIPE Algorithm}

The PIPE method requires the following in order to make its predictions: the sequences of the two query proteins (proteins $A \& B$ having lengths $n$ and $m$, respectively), a list of proteins known to interact as well as their amino acid sequences. The list of known interactions should be made up of high quality physical protein interactions, typically verified by multiple sources, to ensure high confidence. The PIPE algorithm works as follows.

- Step 1: Convert the list of known interactions into a graph $G$, where the proteins are represented as nodes in the graph and the interactions are represented as edges.

- Step 2: Protein $A$ is investigated to find similar proteins within the graph $G$. To do this, protein $A$ is split up into overlapping fragments of size $w$ and these fragments are compared to all sub-sequences of the same size in all of the proteins within $G$. This can be thought of as using a sliding window of size $w$ across protein $A$ and the proteins in $G$. When comparing $A$ against a protein $V$ from $G$, the window starts at the start of both $A$ and $V$. These windows are compared and then the window on $V$ is slid down by one amino acid, creating 
a new fragment to compare. This continues until the first window of $A$ is compared to all of $V$. At this point the window in $A$ is advanced by one amino acid and the process is repeated. This process is repeated for each protein in $G$ until all pairs of windows are compared where none were considered a match, or until a matching pair of windows are found. In the case of a match, all of the proteins adjacent to this matching protein (ie. all of the proteins which are known to interact with protein $V$ ) are put into a list $R$. To determine whether two windows match or not, the PAM 120 matrix [55] is used. This matrix gives a similarity score for all possible pairs of amino acids. An alternative to the PAM matrix could have been to use the BLOSUM matrices [107]. They are inferred from conserved regions of proteins from distantly related organisms, and the observed substitutions are likely a conservatively biased subset of possible mutations. On the other hand, the PAM120 matrix is more inclusive and was therefore chosen here. The windows are simply lined up and the scores for each pair of amino acids are summed. If this score is above a tunable threshold, the windows are considered a match. This threshold was set at an appropriate level (35) to ensure that matches are truly meaningful, with the chance of two random windows matching being less than $10^{-6}$. The size of the sliding window $(w)$ was also experimented with and a window size of 20 was found to be a suitable balance between too small and too large.

- Step 3: A sliding window is again used to compare protein $B$ to all of the proteins in the list $R$, exactly as was done in the previous step. At this point an output matrix of size $n \times m$ is initialized to contain only zeroes. Let $A_{i}$ represent the window on protein $A$ starting at position $i$. Each time a match is found between a window $B_{j}$ in protein $B$ and protein $X$ in $R$, where protein $X$ is known to interact with protein $V$ which had a window which matched the window $A_{i}$, the position $M[i, j]$ is incremented.

- Step 4: The output matrix is visualized as a 3D landscape. This visualizes how often pairs of amino acid sequences were found within proteins that are known to interact and where they are located within the query proteins. Based on this landscape the prediction is made. In this version of PIPE, if there was a cell in the matrix with a value above a tunable threshold $M$, proteins $A$ and $B$ 
were predicted to interact. Here a value of 10 was used for $M$ and it was shown that the chance of two random protein sequences being predicted to interact was less than $10^{-6}$. Figure 3.1 gives an example of a pair of proteins predicted not to interact as well as a pair predicted to interact.

For a summary of the PIPE method, refer to Figure 3.2.

\subsubsection{PIPE Version 1 Overall Results}

This version of PIPE worked exclusively with proteins from the Saccharomyces cerevisiae organism, more commonly known as yeast. This section will highlight some of the scientific results produced by the first version of PIPE.

\section{Ability of PIPE to correctly identify interacting and non-interacting pairs}

To assess the accuracy of PIPE, two sets of protein pairs were assembled. The first consisted of 100 pairs of proteins that are known to interact based on at least three different lines of experimental evidence and were not a part of the set of known interactions PIPE used to make its predictions. The second set were pairs that were expected not to interact based on protein localization data, co-expression profiling, known direct or indirect functional or genetic relationships and the information gathered from the complete set of protein interaction data sets. PIPE classified $61 \%$ of the first set correctly (false negative rate of 39\%) and $89 \%$ of the second set correctly (False positive rate of $11 \%$ ). Together this leads to an overall accuracy of $75 \%$.

\section{Ability of PIPE to Detect Interaction Sites Between Interacting Protein Pairs}

To see if, based on the output matrix, PIPE could predict the actual site of interactions between two proteins, ten pairs for which the interaction sites were know were gathered. Of these 10 pairs, PIPE predicted that seven of them would interact. Of the seven positively predicted interactions, PIPE reasonably detected, based on the peaks in the output landscapes, the interaction sites for four of the pairs. This represents a $40 \%$ success rate for PIPE to successfully predict PPI interaction sites. The available data at the time was minimal and this would be expanded in the future. 
(a)

\section{Hits}

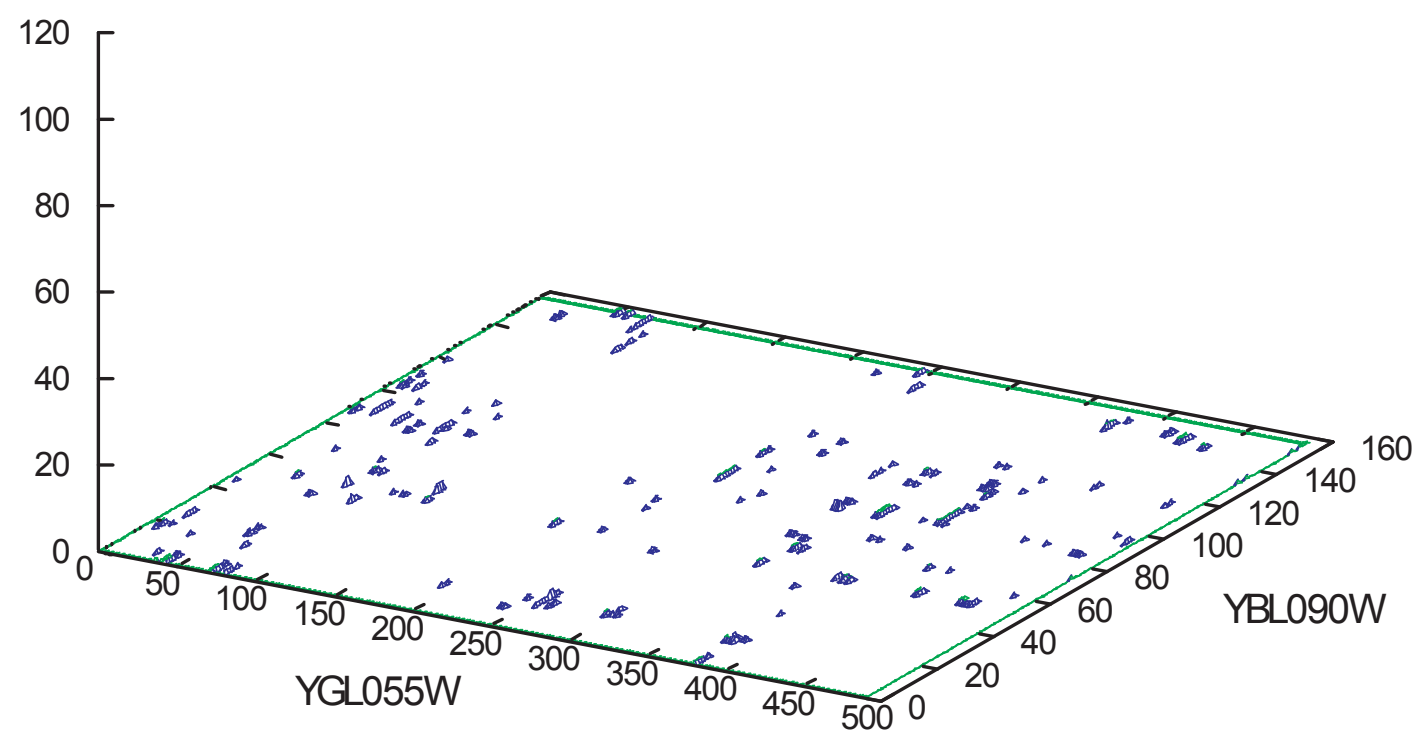

(b)

\section{Hits}

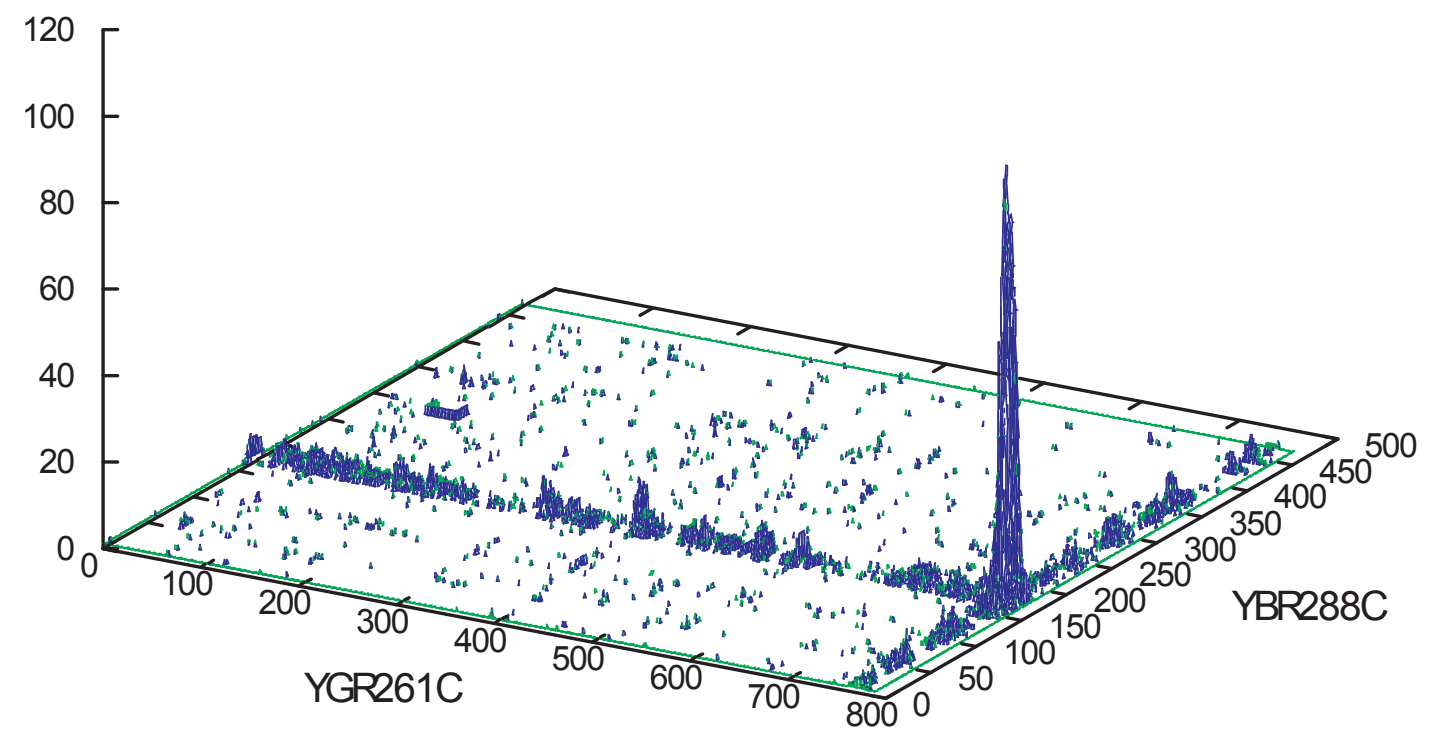

Figure 3.1: Examples of PIPE outputs. The visualization of the output matrix in (a) shows two proteins that are predicted not to interact while the proteins making up matrix (b) are predicted to interact (taken from [224]). 


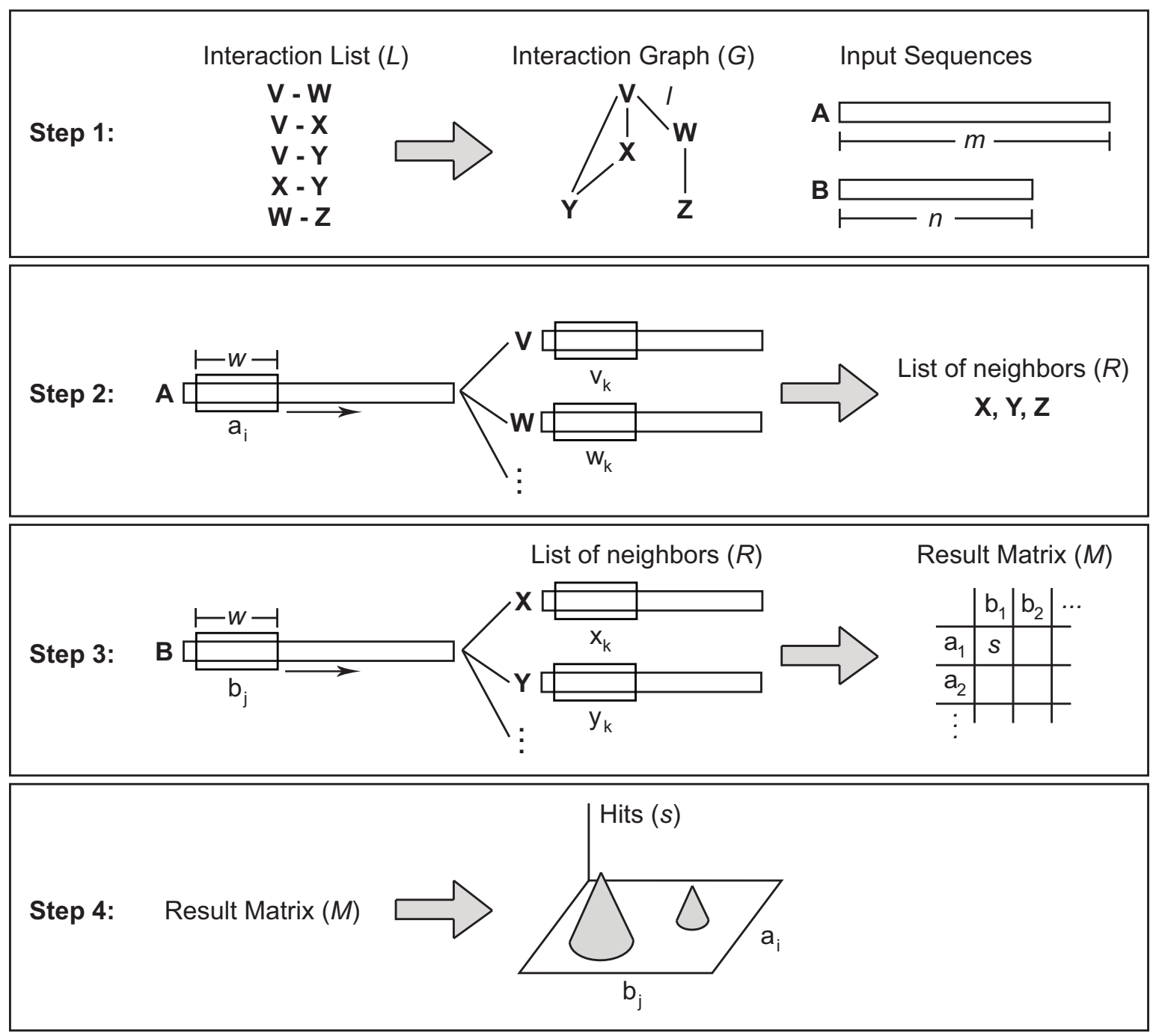

Figure 3.2: An overview of the PIPE Algorithm (taken from [224]).

\section{Ability of PIPE to Detect Novel Protein-Protein Interactions}

To test if PIPE could successfully predict novel PPIs, the proteins YGL227W and YMR135C were investigated. At the time, this pair was not reported to interact in the literature and no interaction data was available. With a peak of nearly 140, PIPE predicted that these proteins would interact (the output matrix can be seen in Figure 3.3). This protein pair was then confirmed to interact in the lab through a method called reciprocal tap-tagging. Interestingly, this technique could only be applied to roughly $31 \%$ of proteins, meaning that this much faster and cheaper PIPE method could also be used to investigate proteins that classical experimental methods could not. 


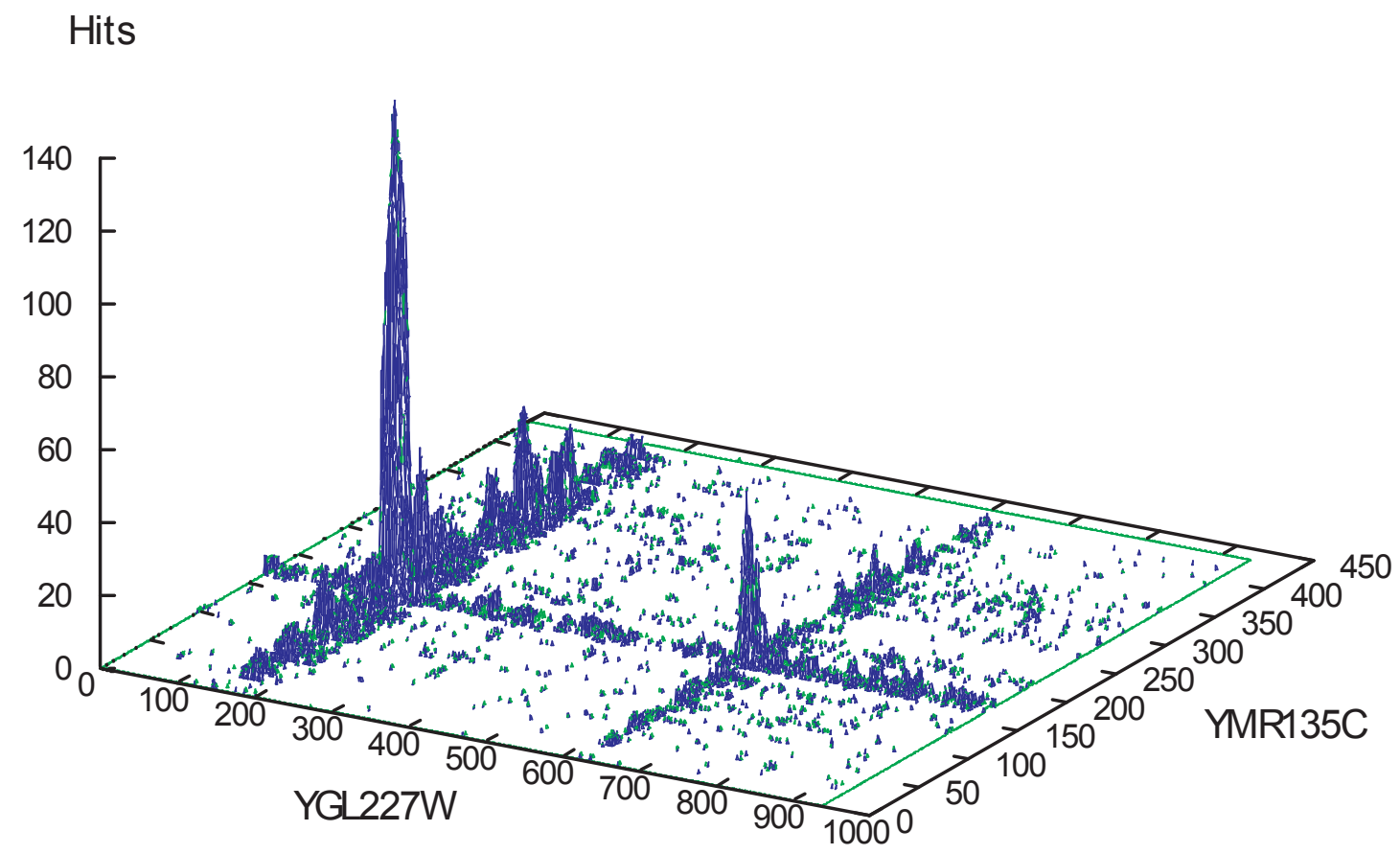

Figure 3.3: Ability of PIPE to detect novel PPIs. Visualized here is the PIPE output matrix for proteins YGL227W and YMR135C. This pair was not reported to interact in the literature and this novel prediction was then confirmed to interact through laboratory experiments. (Taken from [224])

\section{Ability of PIPE to Elucidate the Internal Architecture of Protein Complexes}

While performing the experimental tests on protein YGL227W, a novel protein complex (a group of related proteins that come together through interactions, usually to fulfill some function) was detected. Unfortunately, the internal structure of this novel complex could not be examined through experimental methods. PIPE was used to build a predicted internal structure, shown in Figure 3.4. Through pair-wise laboratory tests, the internal structure of this novel complex was verified. To further test PIPE's ability to investigate protein complexes, 10 known complexes were tested. Out of these 10 complexes there existed 30 potential protein pairs that could interact. Of these 30 pairs, 20 of them were shown to interact while the other 10 didn't. PIPE successfully predicted 13 of the 20 interacting pairs and 4 of the 10 non-interacting pairs. Overall, PIPE predicted the internal structure of three of the complexes perfectly. 


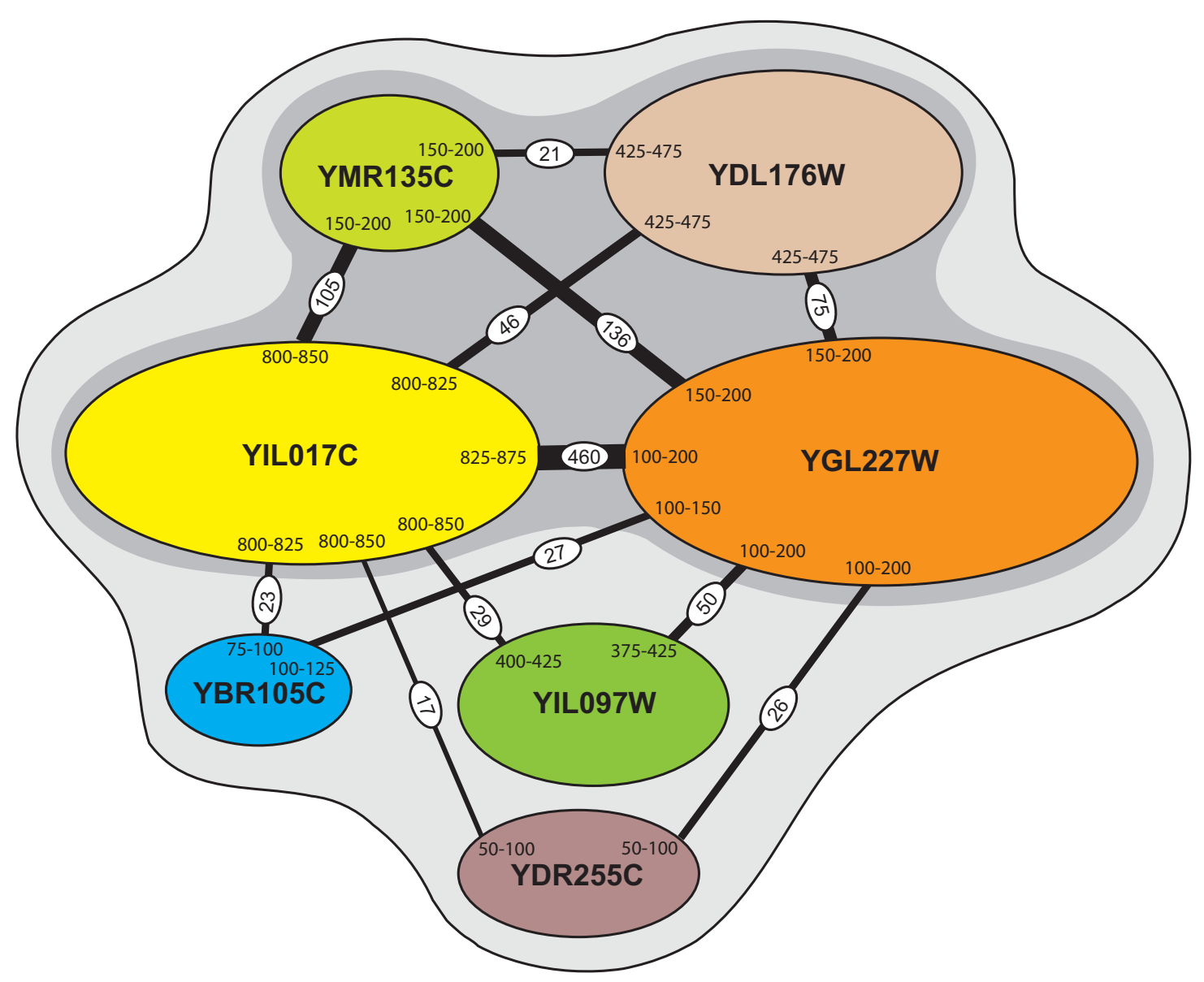

Figure 3.4: Internal structure of a novel protein complex as predicted by PIPE. The various PIPE scores label the edges while the ranges of the predicted interaction sites (based on the PIPE output matrix) are also noted (taken from [224]).

\section{$3.2 \quad$ PIPE2}

The first version of PIPE was shown to be an effective tool to predict protein-protein interactions. With a sensitivity of $61 \%$ and a specificity of $89 \%$, this version of PIPE was shown to produce scientifically relevant results which could be used to augment laboratory approaches when dealing with proteins that are especially difficult to study using traditional techniques. Although PIPE performed relatively well compared to other approaches at the time, it had fundamental weaknesses that would not allow it to make proteome-wide predictions (ie. predict whether or not every possible pair of proteins interact within a given species). A new goal of producing a proteome-wide, all-to-all PPI prediction within the organism Saccharomyces cerevisiae was created. To accomplish this goal, two major roadblocks would need to be addressed. 
The first major issue plaguing the first version of PIPE was the running time. Looking back over the algorithm, it is obvious that a significant amount of work needs to be done to make a single prediction. At the time, it would take several hours on a standard desktop machine to process a single protein pair to make a prediction. These running times would make a proteome-wide, all-to-all prediction within a reasonably sized organism such as $S$. cerevisiae impossible.

The second major issue that needed addressing if PIPE was to perform proteomewide, all-to-all predictions was the low specificity/high false positive rate. Although a specificity of $89 \%$ seems acceptable, when this is scaled up to proteome-wide levels it becomes an issue. For example, S. cerevisiae has roughly 20,000,000 total possible protein pairs and, for argument's sake, let's assume there are roughly 50,000 pairs which are expected to actually interact. Given the performance results from the first version of PIPE, PIPE would be expected to correctly report 30,000 of the 50,000 pairs known to interact as well as correctly identify $17,750,000$ of non-interacting pairs. However, PIPE would also report 2,200,000 false positives and 20,000 false negatives. This would mean that the vast majority of the protein pairs predicted to interact would be false positives and would make the results more or less worthless.

In this section the second version of PIPE that was created to solve these two main issues will be summarized (as described in [226]).

\subsubsection{Improving PIPE's Running Time}

PIPE was initially designed to predict whether a given pair of proteins interacted or not, and a given prediction could take hours to make. Even using a parallelized version of PIPE, it was estimated that a proteome-wide scan of S. cerevisiae would take on the order of 50 years to complete. Because of this, significant improvements would have to be made to PIPE's core algorithm to realize the goal of a proteome-wide scan. There were three major algorithm improvements made to reduce the overall running time.

1. The character-based representation of the amino acid sequences for each query protein were converted into binary, decreasing the time needed to compare window fragments by removing the character-to-index mapping function needed when adding up the PAM120 scores. This improvement produced a speedup of 
18x on average for a set of 1,000 randomly chosen protein pairs.

2. The process of "sliding the window" when comparing proteins was improved. It was noticed that after sliding the window on one of the query proteins, many of the individual amino acid comparisons were identical to the previous window comparison. To address this, the previous window comparison was simply updated to reflect the changes in the new window comparison. Doing this lead to a speedup of $43 \mathrm{x}$ on average for a set of 1,000 randomly chosen protein pairs.

3. The fact that a lot of identical protein fragment comparisons were repeated during the computation of the PIPE algorithm was the third item addressed. Two steps were needed to remedy this situation. Firstly, all of the proteins making up the known interaction graph as well as all potential query proteins were broken up into their overlapping fragments, all unique fragment pairs were compared and all of the pairs that were deemed to match were recorded to disk. A database of these records was constructed. When a set of query proteins are presented to the system, the relevant database files can be loaded into main memory. This data tells PIPE the set of proteins that contain a matching fragment at each window location. This completely removes the need for PIPE to calculate any fragment comparisons while running. This step removed an enormous amount of redundant work being performed by PIPE. For each protein PIPE now has access to a 'similarity file' which records all of the proteins that contain a similar window for all windows of the protein in question. Performing the lookup as opposed to computing how similar two fragments were produced a speedup of 16,150 times on average for a set of 1,000 randomly chosen protein pairs.

These speedups are summarized in Table 3.1.

One thing that should be noted is that the speedup times above do not include the pre-computation time needed to produce the PIPE database files. Relatively speaking, this pre-computation time was rather insignificant ( 30 minutes or 1\%) compared to the total time needed to run the entire proteome-wide, all-to-all prediction ( $\sim 8$ hours on 76 processors). When taking this into account, the above improvements lead to a speedup of $14,775 x$. 
Version

Average Runtime (s) Speedup

Original PIPE implementation

6944.40

$1 \mathrm{x}$

+ Digital alphabet optimization

389.65

$18 \mathrm{x}$

+Sliding window optimization

160.53

$43 \mathrm{x}$

+Pre-computation/Query approach

$0.4316,150 \mathrm{x}$

Table 3.1: Performance improvements from PIPE1 to PIPE2 [226].

Although the end product of the core PIPE algorithm (as described in Section 3.1.1) remained the same, the introduction of this pre-computed database of similarity files altered the main PIPE algorithm. For two query proteins $A$ and $B$ (of lengths $n$ and $m$, respectively), the altered algorithm works as follows.

- Step 0: Before PIPE2 can be run for a given query protein pair, the precomputation (as described above) must be done.

- Step 1: Step 1 remains unchanged from the previous version.

- Step 2: The second step of the PIPE algorithm is completed by simply loading the similarity files of both proteins into memory. For each sliding window location along proteins $A$ and $B$ there is a list of proteins that contain a window deemed similar. The output matrix of size $n \times m$ is also initialized to contain all zeroes. Steps 3 and 4 are repeated for each window location on protein $A$.

- Step 3: For each window location in protein $A$, there is a list $\left\{A^{\prime}\right\}$ of "similar" proteins from the similarity file loaded in step 2. For each protein $A^{\prime}$ in $\left\{A^{\prime}\right\}$, a list $\left\{B^{*}\right\}$ of proteins known to interact with $A^{\prime}$ is retrieved from the known interaction graph.

- Step 4: For each protein $B^{*}$ in $\left\{B^{*}\right\}$, every window location on protein $B$ is examined. For each window location, a list $\left\{B^{\prime}\right\}$ of "similar" proteins is retrieved from the similarity file loaded in step $2 .\left\{B^{\prime}\right\}$ is then scanned to see if $B^{*}$ appears in the list. If so, this represents a match and the output matrix is incremented at the respective window locations on proteins $A$ and $B$.

- Step 5: The output matrix is analyzed as before to determine if proteins $A$ and $B$ are predicted to interact. 
Although this is an alteration to the way the PIPE algorithm previously ran, one should see that, as before, all possible window combinations on proteins $A$ and $B$ are analyzed and the output matrix is updated as before, meaning the end result of using either version of the algorithm remains identical for any given query protein pair. Since this means that the two algorithms are equivalent, this modified version could be used in place of the previous version.

\subsubsection{Improving PIPE's Predictive Performance}

The second major issue that needed to be addressed was the number of false positives that PIPE produced. The first step in addressing this was to apply a median filter to the output matrix. The goal in doing this was to eliminate tall, thin peaks (which are viewed as random anomalies that could lead toward a false positive prediction) but would leave larger hill regions with significantly large bases. However, applying this filter to the output matrix was very computationally expensive, taking longer than running the new PIPE algorithm itself. To combat this, the filter was modified so that a given cell was set to 0 if most of its neighbours were zero and 1 otherwise. After this filter was applied, there were no longer any peaks to determine if the pair of proteins would be predicted to interact. The average cell value was calculated and if this value was above a certain threshold, the pair of proteins would be predicted to interact. After a series of tests, a filter size of $3 \times 3$ was found to be reasonable. To see how this filter would eliminate potential false positives, see Figure 3.5.

\subsubsection{Leave-One-Out Cross-Validation tests}

To test the statistical predictive performance of PIPE2, leave-one-out cross-validation (LOOCV) tests were performed. To do these tests, a positive set (set of proteins known to interact) and a negative set (set of proteins known not to interact) are needed. In this case, 1,274 known $S$. cerevisiae interactions were taken from the intersection of data retrieved from BioGRID [37], which is a curated database of high-quality protein-protein interaction data taken from a variety of sources, and a recently published, high-throughput experimental study [143]. Building negative sets for protein-protein interactions is not as straight forward. There is not a vast amount of known, non-interacting proteins and, for this reason, a set of assumed 
Before filter:
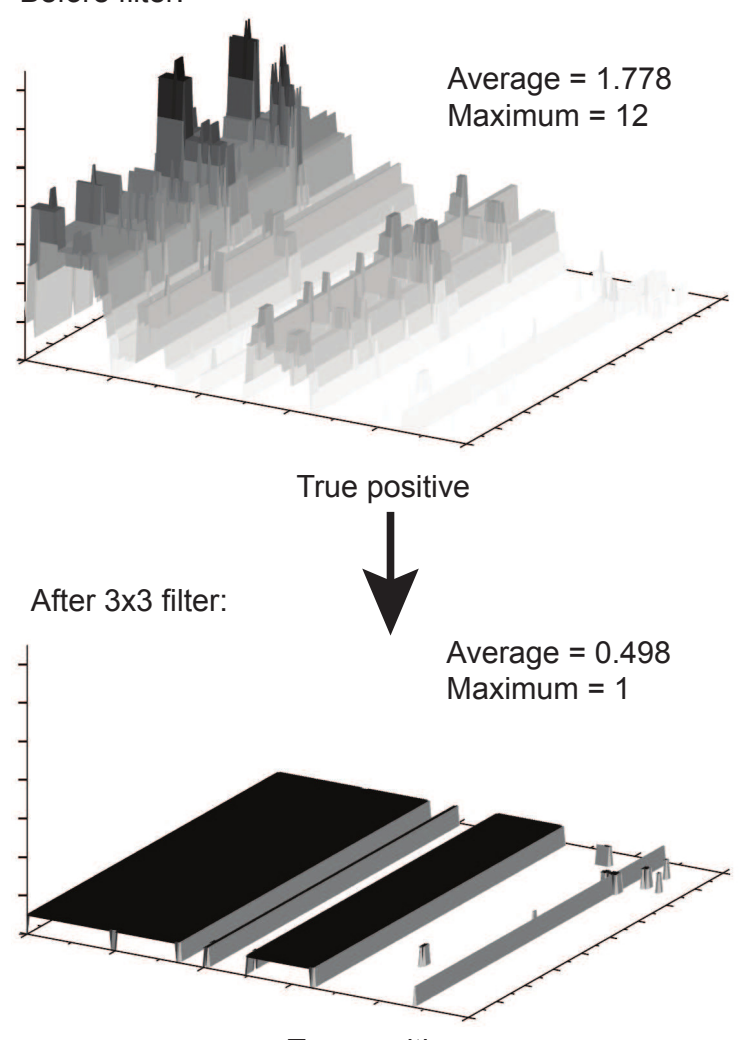

True positive
Before filter:

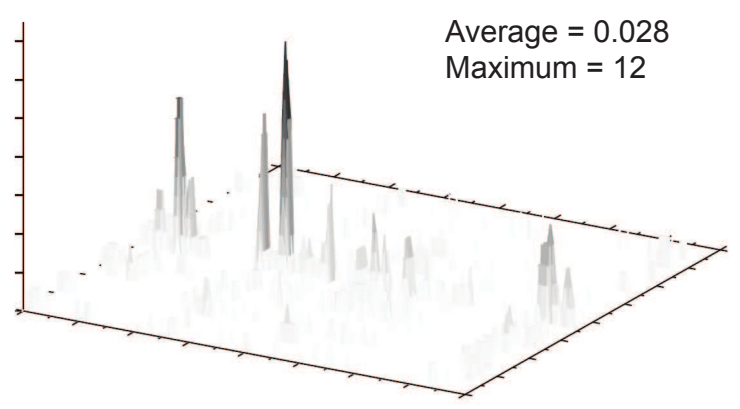

False positive

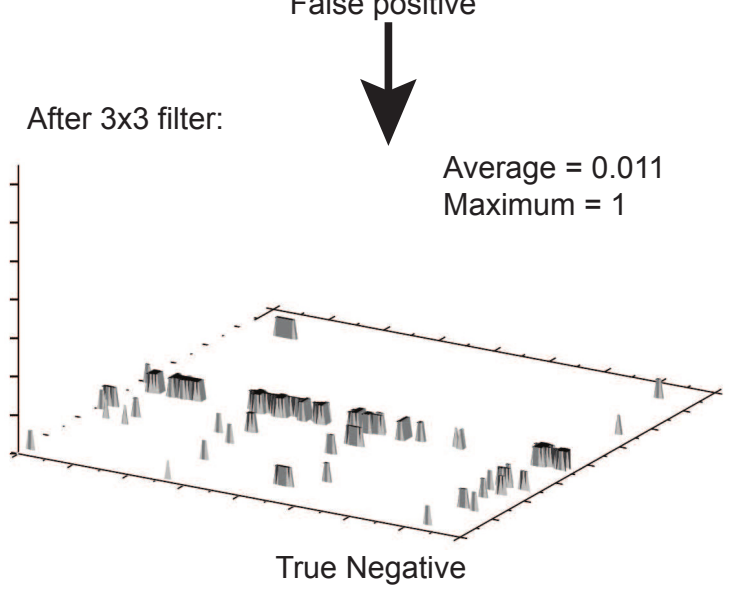

Figure 3.5: The effect of the modified median filter on true and false positive PIPE outputs (taken from [226]).

negative interactions must be generated. It has been shown in other studies $[19,211]$ that imposing some restrictions on how the negative pairs are chosen (ie. choosing proteins that do not share the same cellular location that are not likely to ever come in contact with one another in nature) leads to a biased representation of the non-interacting protein pairs within a given organism. The goal in constructing the negative set should be to give a sample representation of the global negative set and adding restrictions on how this set is built will skew this representation. To combat this problem, a very simple scheme of choosing negative protein pairs uniformly at random has been suggested. It has been noted that building a negative set in this fashion can lead to contamination with positive pairs, however since the estimated number of interactions within an organism is so incredibly low (a fraction of a percent of the total possible protein pairs), this kind of contamination will have a negligible 
impact $[19,211]$. With this in mind, a negative set of 100,000 protein pairs were chosen uniformly at random and were checked to ensure it contained no pair from the positive set.

Now that the positive and negative sets have been established, the LOOCV tests could be run. For each pair in the positive set, prior to the individual test, it is removed from the PIPE2 database. This puts PIPE2 into a state in which it has no idea that the pair is known to interact, and then tries to predict whether it is an interacting pair or not. Each pair is tested individually, meaning only the pair being tested is removed from the database. Once the positive set is completed, predictions are then run on the negative set. In this case however, nothing needs to be removed from the database since the negative set contains no known interactions. Once both sets have been run, they are put together and then sorted by their PIPE scores. This then allows one to choose an operating point for PIPE by setting the threshold above which a pair is predicted to interact. Once this threshold is set, one can then count how many true positives (TP), true negatives (TN), false positives (FP) and false negatives (FN) would occur using this threshold. Typically, a threshold that achieves an extremely high specificity is chosen to ensure that the likelihood that positive prediction is not a false positive is extremely low. The measures of sensitivity (TP $/(\mathrm{TP}+\mathrm{FN}))$ and specificity $(\mathrm{TN} /(\mathrm{FP}+\mathrm{TN}))$ will be used here since many other statistical measures are heavily influenced by the relative size of the positive and negative sets. When using these other statistical measures, the chosen size of the negative set can directly influence the stated statistical performance, unlike the measures chosen here.

Once these LOOCV tests were carried out on the above described positive and negative sets, a suitable threshold was chosen. At this threshold, PIPE2 correctly predicted 99,946 of the negative set would not interact, leaving 54 false positives. PIPE2 also correctly classified 186 of the 1,274 known positive pairs, leaving 1088 false negatives. Therefore, at this chosen threshold, PIPE2 was able to obtain a specificity of $99.95 \%$ and a sensitivity of $14.6 \%$. Although this sensitivity is seemingly low, it allows PIPE to be quite confident that any pair predicted to interact is not a false positive. These thresholds can be adjusted to allow for a higher sensitivity, however an extremely high specificity is necessary for a proteome-wide, all-to-all prediction. 
Unless otherwise stated, the manner in which the LOOCV tests were described above is how they were performed on all data sets described in this thesis.

\subsubsection{PIPE2 Overall Results}

The main goal of PIPE2 was to produce a proteome-wide, all-to-all prediction in the S. cerevisiae organism. The following statistics represent the available data at the time.

- total number of yeast proteins: 6,304

- total number of proteins with at least one known interacting partner: 4,716

- total number of proteins with no known interacting partners: 1,588

- total number of possible interactions: $19,867,056$

- largest number of known interactions partners for a single protein: 282

- smallest number of known interactions partners for a single protein: 0

- total number of known interactions: 15,118

- average number of known interactions per protein: 4.80

- average number of known interactions per protein (w/ at least one interaction): 6.41

PIPE2 was run on all 19,867,056 possible $S$. cerevisiae pairs and identified 29,589 positive interactions. Of these predicted interactions, 15,151( 51.2\%) were previously known interacting pairs, leaving 14,438 pairs as novel predictions. To investigate the validity of these novel predictions, three sets of 100 novel pairs were gathered and were studied to identify common functional information for each pair or to see if the two proteins also shared a third party interaction. These lines of evidence give added support to the validity of PIPE2's novel predictions. The results of this analysis is summarized in Table 3.2.4.

Further study was done on proteins known to be involved with non-homologous end-joining (NHEJ, a DNA repair mechanism). A novel prediction containing a protein involved in NHEJ was studied and later confirmed through wet lab experiments, which showed that the second protein as well as the interaction were also involved in this process. This shows that biologically meaningful information can be mined from the novel predictions made by PIPE2. 


\begin{tabular}{lcccc}
\hline Set Number: & 1 & 2 & 3 & Total \\
& & & & \\
\hline & & & & \\
Evidence Based On Function & 20 & 22 & 17 & 59 \\
Evidence Based On Third Interaction & 49 & 45 & 46 & 140 \\
Both Lines of Evidence & 17 & 13 & 9 & 39 \\
Either Line of Evidence & 69 & 67 & 63 & 199 \\
& & & & \\
\hline
\end{tabular}

Table 3.2: Analysis of PIPE2 data using common functional relationships as well as the presence of a common third party interactor to support the novel PIPE2 predictions [226].

\section{$3.3 \quad$ MP-PIPE}

Although PIPE2 was a vast improvement on the original version of PIPE, it still had major deficiencies. It became clear that although PIPE2 could perform proteomewide, all-to-all predictions for organisms like S. cerevisiae, it could not process more complex organisms such as Caenorhabditis elegans and Homo Sapiens. The main reason for this was that although PIPE2 ran in an embarrassingly parallel manner, no attempt was made to optimize how multiple instances of PIPE2 ran at the same time. To run PIPE2 on a given cluster, each instance was given a static list of protein pairs to make predictions on. This parallelization scheme has two inherent flaws. Firstly, there is no load balancing done whatsoever. Although most protein pairs can be processed by PIPE2 in a fraction of a second, there are a small number of harder pairs that can take orders of magnitude longer. The reason for this is that some proteins have segments that match very high degree proteins in the known interaction graph. This leads to a significant amount of work to then compare the second protein to all of the neighbours of the matching protein. The extent to which this happens has a very large impact on the overall running time, which leads to a small percentage of pairs taking an exceedingly long time to process. The problem with this range of running times for protein pairs is there is no way to predict how long a given pair will take to process. Since each PIPE2 instance was simply given a random subset of the overall protein pairs to process, some instances would get relatively easy protein pair lists to process, would finish relatively quickly and then 
simply idle while the other processes continued working. In some more extreme cases the total idle time could actually eclipse the total working time. This happens when other PIPE2 instances get unusually hard protein pair lists to process, causing them to need to spend a significantly longer time working while the majority of the other instances idled. In an ideal situation there would be some mechanism in place to ensure a more balanced workload between all of the PIPE2 instances.

The second PIPE2 flaw was the more pressing of the two: on most systems PIPE2 was not able to fully utilize all available computing resources due to poor memory management. Since there was no communication at all between PIPE2 instances, no data could be shared between them, even on instances running on the same physical compute node. This meant that each instance would need to load its own instance of the known interactions graph on top of the needed database files, the intermediate and result matrices and all other PIPE2 related data needed to perform its predictions. This was simply too much when dealing with more complex organisms and in some cases the number of instances per compute node had to be scaled down significantly to give each instance enough memory or in other cases it would simply not work at all.

Both of these issues were addressed in the third version of PIPE, MP-PIPE (Massively Parallel PIPE). This section describes how MP-PIPE dealt with the above mentioned issues and the biological results it produced (as described in [250, 253]).

\subsubsection{New Parallel Architecture of MP-PIPE}

To address the inherent flaws of PIPE2, a new parallel architecture was designed and implemented. This architecture had two keep components, each component addressing one of PIPE2's major flaws. The first component was the introduction of a master-slave model for work distribution. In this model there would be a series of PIPE slave instances who would all request work from a single master PIPE process. This master process would hold all of the work needed to be done (the entire list of protein pairs to process) and would send this work out to slave processes in relatively small packets. These slave processes would then work through this packet of work, send the results back to the master process which in turn would act as a request for more work. This work flow would continue until the master process runs 
out of work. Once this happens, the master process simply returns a stop signal to the slave processes upon a work request. The program finishes once all of the slave processes acknowledge that they have received their stop signals. This scheme nearly completely removes the load imbalance issue. The reason for this is that all of the slave processes are only given a relatively small amount of work to do at a time, meaning that no single process will get bogged down for an overwhelming amount of time while the other processes sit idle. Even if a slave process is given a relatively hard packet of work, it will continue to work while other processes finish their packets, receive new ones and continue working. The only time that a given slave will not be working while other slaves are is when the master process has no more work to distribute. However, when the amount of work increases and the packet size is small enough, the amount of wasted resources becomes negligible. One should note that although the packet size is a parameter that can be chosen at run time, a packet that is too small will cause an increase in communication overhead. On top of the overhead incurred by creating, sending, and waiting for responses from messages, the master process could become overwhelmed if too many slave processes ask for work at the same time. For this reason it is important to find the right balance between too large of a packet (causing idling) and too small of a packet (increase communication overhead).

The second component of the new parallel architecture of MP-PIPE was the introduction of an all-slaves model incorporated into the slave processes. In this model, the slave process would start out as a single computational thread, would set up any data structures and load any data from files needed and then would split up into a user-defined number of computational threads. These threads would then all work in parallel in the following manner. First, one of the threads would realize that there is no work currently available and would send a request to the master process. If the master process returns a valid packet of work, the thread would set that packet up for the other threads to work on. All of the threads would then take a protein pair from the packet to process in parallel. Once the entire packet has been processed, one of the threads sends the result to the master process and requests more work. If the master responds process with the signal that there is no work remaining, all of the parallel computational threads collapse back into a single thread which then 
waits for the rest of the slave processes to finish before exiting. One major benefit of this approach is that all of the threads exist in a shared-memory environment, so all of the information that can be shared between independent PIPE processes would be shared instead of having multiple instances of the same information in memory. An example of this is the relatively large interaction graph which now could now be shared across all of the threads instead of being redundantly stored multiple times in memory. On top of this, the pre-computed database files were not all loaded at once at the start of the computation as they were in the PIPE2 implementation, they were loaded only when they were needed. This allowed more threads to run simultaneously on a given cluster node by reducing the overall memory usage. These aspects of the newly introduced all-slaves computational model, the on-demand database file loading and a general overhaul of the overall memory usage of PIPE allowed more threads to run simultaneously on a given cluster node by reducing the overall memory usage.

Once both of these components were put together it allowed MP-PIPE to successfully overcome the two major faults of PIPE2. This architecture also allowed MP-PIPE to adapt and scale to any physical compute cluster, be it a small cluster made of a handful of desktop PCs or a massive compute cluster made up of independent nodes with a large number of hardware supported threads. The general approach when running MP-PIPE was to place once slave process on each physical compute node. Each one of these slave processes would then be set to have enough compute threads to fully utilize all of available hardware supported threads. The master process would then be placed alongside one of the slave processes. This did not have a large impact due to it only needing one compute thread to carry out its work of packet distribution.

\subsubsection{MP-PIPE Benchmarking}

To benchmark MP-PIPE, two different tests were designed. The first test was designed to measure the impact that the number of compute threads had on processing time of a single slave process. MP-PIPE was set up to run with a single master process, a single slave process and a fixed amount of work. The slave process would start with a single thread which was then subsequently raised until an optimal number of threads was found. The second test was designed to show how well MP-PIPE scaled 
when more compute nodes (and, by extension, slave processes) were used. In this test a fixed amount of work was used and each slave process used the optimal number of threads as found in the first test.

These tests were then carried out on three different compute clusters. The first compute cluster was the Carleton Bioinformatics Lab Cluster. This cluster was made up of 8 desktop PCs with Intel Quad Core $(1.66 \mathrm{GHz})$ processors and 8 GB of RAM each interconnected with a gigabit switch. The second cluster used was the High Performance Computing Virtual Laboratory (HPCVL)'s Beowulf Cluster, made up of 64 nodes with 4 Opteron Cores $(2.2 \mathrm{GHz})$ and 8 GB RAM each interconnected by a gigabit switch. The third cluster used was HPCVL's Victoria Falls Cluster, made up of 73 nodes with 8 compute cores having a total of 128 hardware supported threads and either 64 GB or 32 GB of RAM each interconnected by a gigabit switch. The tests described above were carried out on each of the three compute clusters described to give an idea of how MP-PIPE would perform on different platforms. The reported results were the average of 100 test runs.

\section{Benchmarking Test 1 Results}

Benchmarking test 1, as described above, was carried out on all three compute clusters. The speedup results can be seen in Figure 3.6.

As can be seen in the speedup plots, MP-PIPE sees a significant performance improvement when increasing the number of threads used up to the total number of compute cores on a given node of the compute cluster being used. The performance plateaus significantly at this point, however a slight improvement when increasing the number of threads past this point is still observed. The general conclusion to take from this test is the optimal number of threads is roughly 1.5 times the number of compute cores, allowing MP-PIPE to gain that extra bit of performance by either utilizing the available hardware supported threads or to hide memory latency. Since the PIPE algorithm has to make a considerable amount of I/O calls (opening the PIPE database files, miscellaneous memory look-ups), the time that a given thread waits for these I/O calls can be hidden by simply swapping this thread out and swapping another thread that is ready to do some processing in. 
Carleton Bioinformatics Lab Cluster Benchmarking Test 1: Speedup
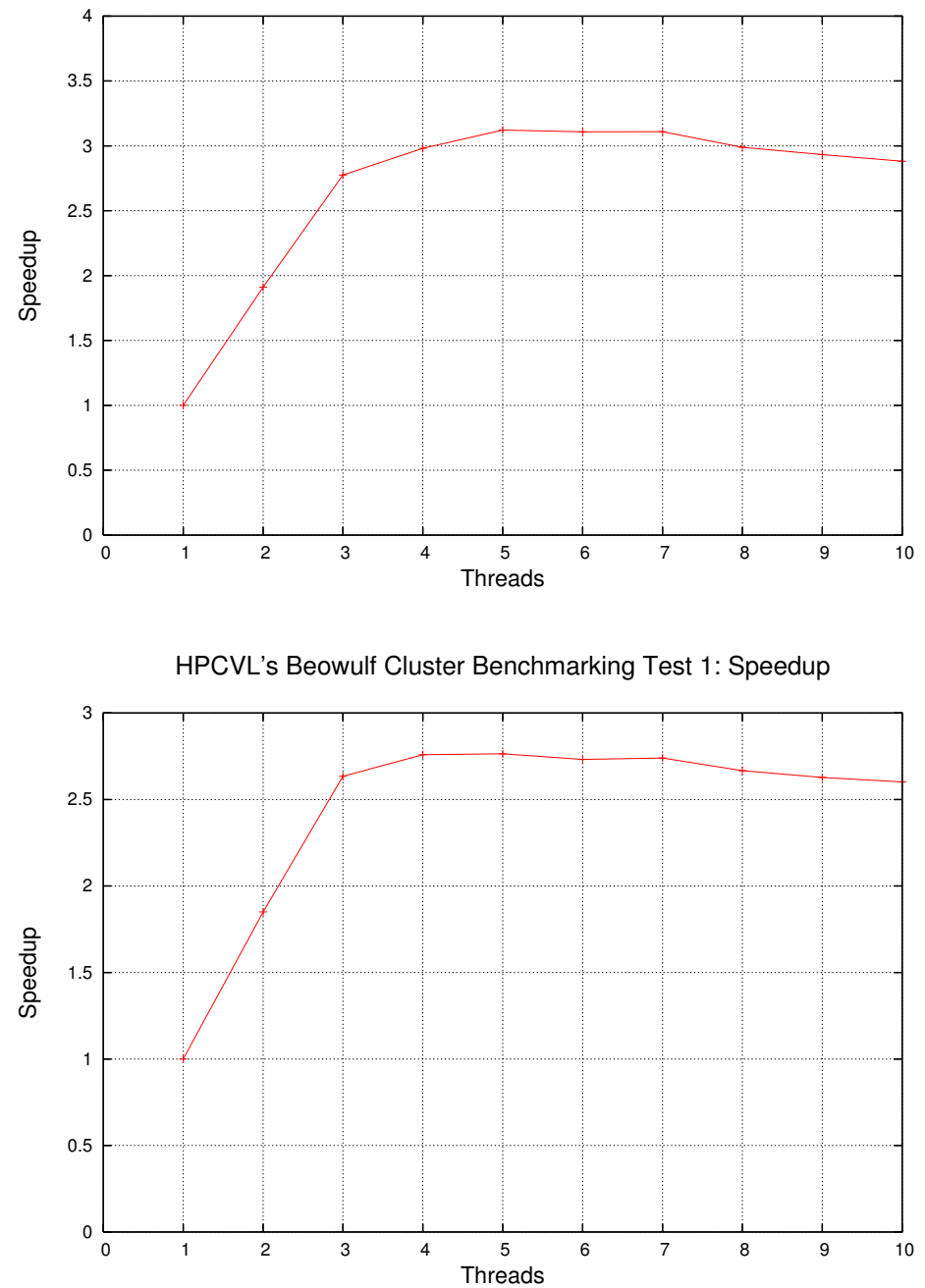

HPCVL's Victoria Falls Cluster Benchmarking Test 1: Speedup

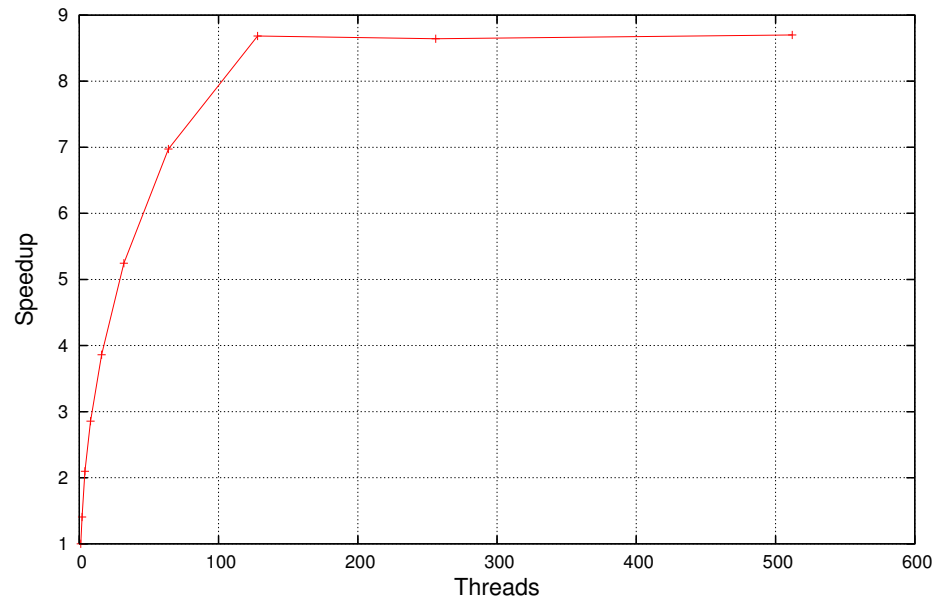

Figure 3.6: MP-PIPE Benchmarking Test 1 Speedup Results (taken from [250]). 


\section{Benchmarking Test 2 Results}

Benchmarking test 2, as described above, was carried out on all three compute clusters. The speedup results can be seen in Figure 3.7.

As can be seen in the speedup plots, MP-PIPE generally sees a linear speedup when increasing the number of slave processes/compute nodes used. The cases where a slight super-linear speedup is observed is an artifact created by the fact that the baseline tests were done with both the master and slave process being placed on the same compute node. This slight hindrance is not present for the other slave processes on the other compute nodes. Generally speaking, one can expect a close to linear speedup when using MP-PIPE.

\subsubsection{Summary of Biological Results Produced by MP-PIPE}

MP-PIPE was then used to perform proteome-wide, all-to-all predictions for the organisms Caenorhabditis elegans and Homo sapiens. These were the most complex organisms tackled by any version of PIPE at the time.

\section{Caenorhabditis elegans Proteome-wide prediction results}

The first organism to be processed was $C$. elegans. At the time this work was carried out, the following statistics represent the data available.

- total number of $C$. Elegans proteins: 23,684

- total number of proteins with at least one known interacting partner: 3,460

- total number of proteins with no known interacting partners: 20,224

- total number of possible interactions: 280,454,086

- largest number of known interactions partners for a single protein: 512

- smallest number of known interactions partners for a single protein: 0

- total number of known interactions: 6,607

- average number of known interactions per protein: 0.55

- average number of known interactions per protein with at least one interaction: 3.82

MP-PIPE was run on all 280,454,086 possible C. elegans protein pairs. This workload was split between the HPCVL's Beowulf cluster and HPCVL's Victoria Falls cluster. 

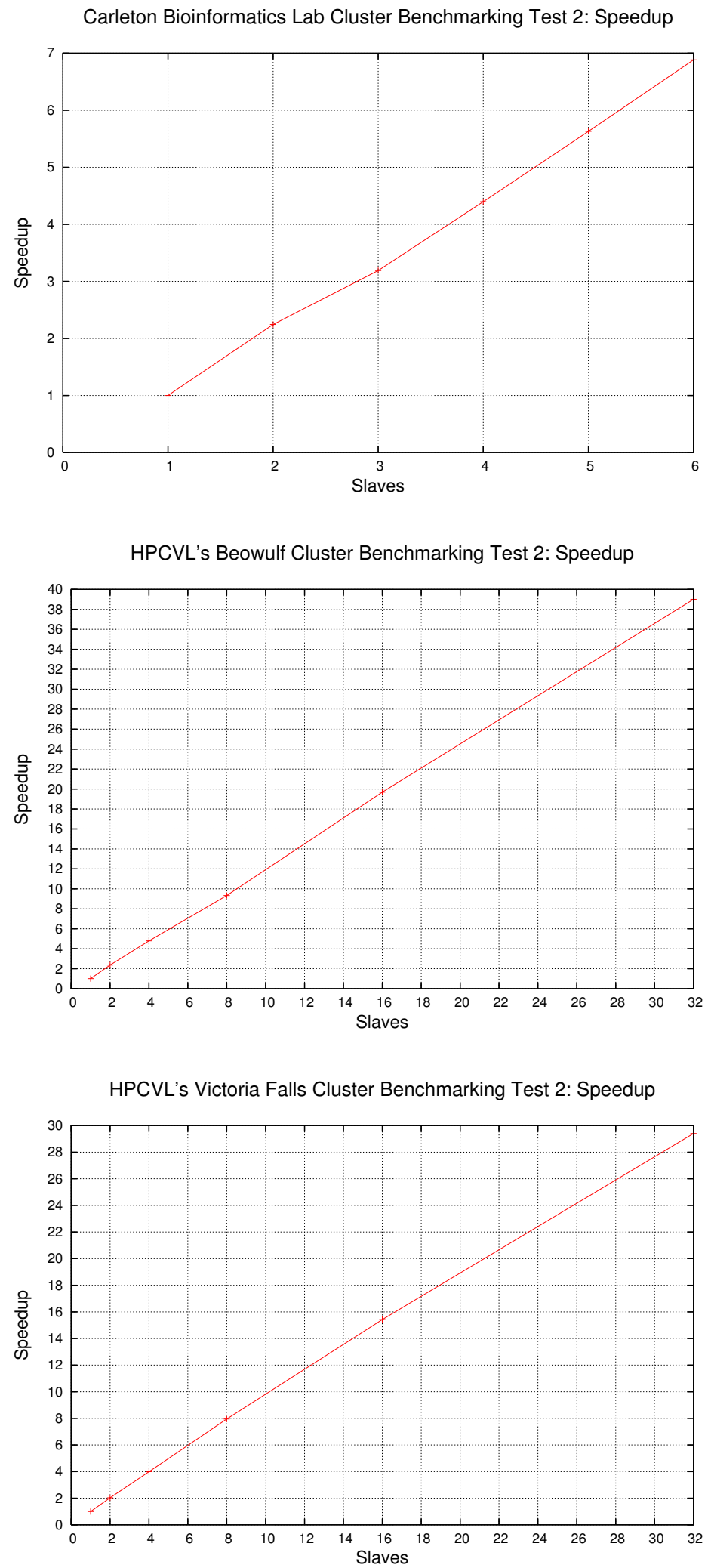

Figure 3.7: MP-PIPE Benchmarking Test 2 Speedup Results (taken from [250]). 
On the Victoria Falls cluster, 50 nodes were used each with their own slave process running 512 threads. This translated into 25,600 parallel computational threads running on 6,400 hardware supported threads, representing the largest job in terms of scale that has ever been run on an HPCVL machine at the time. On the Beowulf cluster, 60 nodes were used each with their own slave process running 8 threads. This translated into 480 parallel computational threads running on 240 cores. The work was split up into jobs of roughly $10 \%$ of the overall data set and took roughly a week to process using both clusters.

Once predictions had been made on all 280,454,086 possible C. elegans protein pairs, leave-one-out cross-validation tests were carried out (in the same manner as described in Section 3.2.3). Using these results, an appropriate accept threshold was established. Using this threshold, PIPE achieved a specificity of $99.99 \%$ and predicted 37,572 interactions, 32,548 of which were novel. To add biological credibility to these results, the protein pairs were grouped according to their molecular function, biological process and location inside the cells (as has been done in previous predictions to add confidence to the results). The percentage of pairs that have similar function, occur in the same cellular component and participate in the same cellular process is $1.3 \%$, which is consistent with the percentage for previously reported protein pairs (1.9\% for 6,607 pairs). In contrast, for randomly selected protein pairs, the percentage of pairs that have similar function, occur in the same cellular component and participate in the same cellular process is only $0.2 \%$ (for 100,000 tested random pairs). A summary of these results can be seen in Figure 3.8.

\section{Homo sapiens Proteome-wide prediction results}

The second organism to be processed was H. sapiens. At the time this work was carried out, the following statistics represent the data available.

- total number of $H$. sapiens proteins: 22,513

- total number of proteins with at least one known interacting partner: 9,459

- total number of proteins with no known interacting partners: 13,054

- total number of possible interactions: 253,406,328

- largest number of known interactions partners for a single protein: 265

- smallest number of known interactions partners for a single protein: 0 
- total number of known interactions: 41,678

- average number of known interactions per protein: 3.70

- average number of known interactions per protein with at least one interaction: 8.81

MP-PIPE was run on all 253,406,328 possible $H$. sapiens protein pairs. At this point the $C$. elegans organism was by far the most complex proteome processed by any version of PIPE. The H. sapiens interactome contained almost 7 times the known interactions than in the $C$. elegans interactome, leading to the average $H$. sapiens protein having more twice the known interactions that the average $C$. elegans protein had. This, coupled with the fact that the average $H$. sapiens protein was longer than the average $C$. elegans protein, would prove to increase the complexity of scanning the entire H. sapiens proteome significantly.

This task was carried out mostly on HPCVL's Victoria Falls cluster. On this cluster 50 nodes were used each with their own slave process running 256 threads. This translated to 12,800 parallel computational threads running on 6,400 hardware supported threads. The reason for lowering the number of threads used per node was due to each thread requiring significantly more memory than the previous runs. Lowering the total number of threads used allowed each thread access to more memory. Due to the complexity of the $H$. sapiens proteome, a small fraction of pairs took an extremely long time to process. Because of this, a 12 hour time limit was placed on any single pair. If this time limit was reached, that pair was offloaded and processed on HPCVL's Beowulf cluster since its individual compute threads are much more powerful than Victoria Falls'. Using this scheme, the entire proteome was processed after roughly 3 months.

Once predictions had been made on all 253,406,328 possible $H$. sapiens protein pairs, leave-one-out cross-validation tests were carried out (in the same manner as described in Section 3.2.3). Using these results, an appropriate accept threshold was established. Using this threshold, PIPE achieved a specificity of $99.95 \%$ and predicted 172,183 interactions, 130,470 of which were novel. To add biological credibility to these results, the protein pairs were grouped according to their molecular function, biological process and location inside the cells (as has been done in previous predictions to add confidence to the results). The percentage of pairs that have similar 
function, occur in the same cellular component and participate in the same cellular process is $50.3 \%$, which is consistent with the percentage for previously reported protein pairs $(67.7 \%$ for 41,678 pairs). In contrast, for randomly selected protein pairs, the percentage of pairs that have similar function, occur in the same cellular component and participate in the same cellular process is only $11.3 \%$ (for 100,000 tested random pairs). A summary of these results can be seen in Figure 3.9.

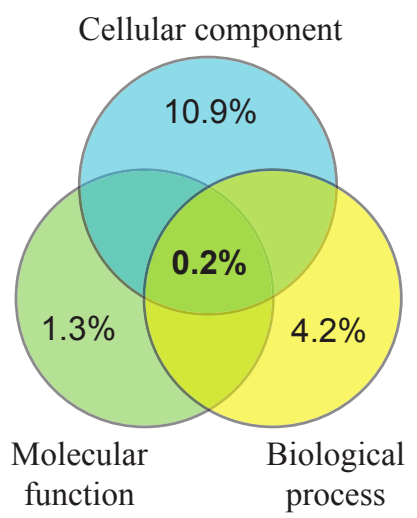

(a) Random Pairs

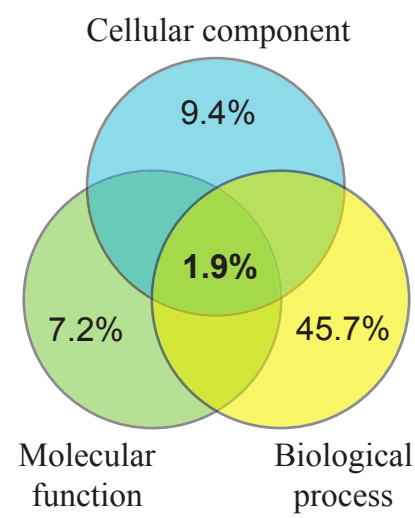

(b) Previously Detected Interactions

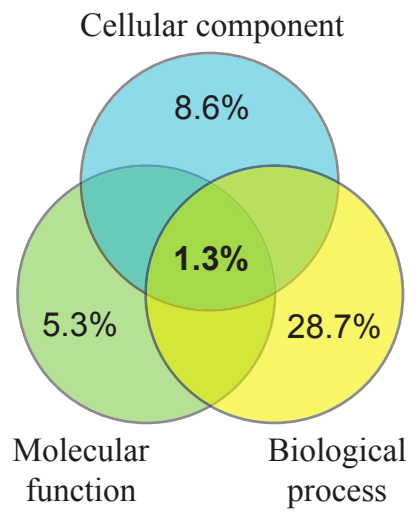

(c) PIPE3 Predictions

Figure 3.8: A comparison of the grouping of random protein pairs, previously detected interactions and MP-PIPE predictions in terms of cellular component, molecular function and biological process for C. elegans proteins. (taken from [250]).

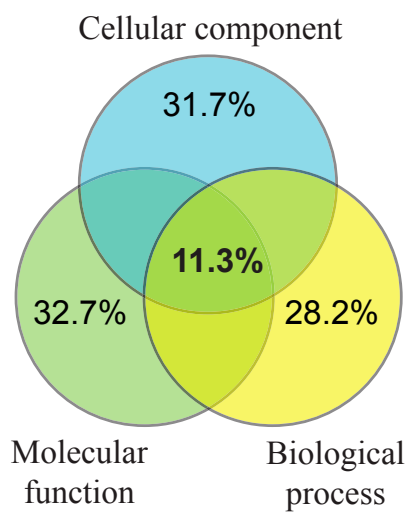

(a) Random Pairs

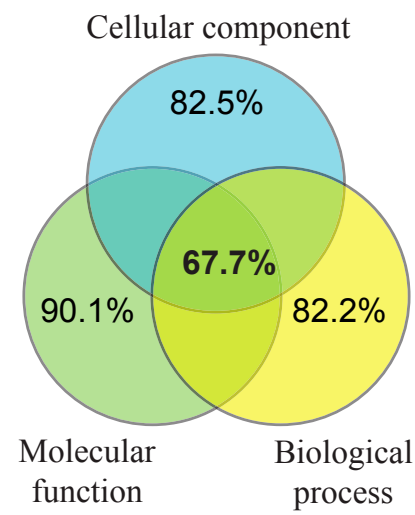

(b) Previously Detected Interactions

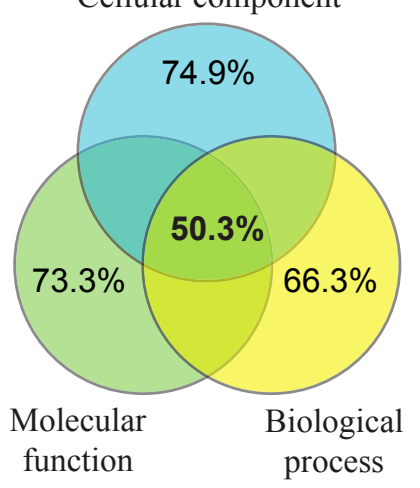

(c) PIPE3 Predictions

Figure 3.9: A comparison of the grouping of random protein pairs, previously detected interactions and MP-PIPE predictions in terms of cellular component, molecular function and biological process for H. sapiens proteins. (taken from [250]). 


\section{Chapter 4}

\section{Further Development and Improvements of PIPE}

Although MP-PIPE was a significant improvement over the previous versions, there were still opportunities to further improve PIPE's performance or extend its capabilities in other ways. Over the next few sections, these novel improvements and extensions will be described. Section 4.1 describes an extension to PIPE named PIPE-Sites which, using intermediary data from the PIPE algorithm, predicts the site of interaction between two proteins. Section 4.2 describes a new scoring method that outperforms the traditional PIPE scoring function (in terms of sensitivity and specificity) as well as further performance improvements. Lastly, Section 4.3 describes the program as it stands now, incorporating all of the stand alone improvements made previously. It should be noted that the author of this thesis isn't directly responsible for all of this work, in fact most of the development and testing were performed by others. However, the author did participate in the study resulting in the PIPE-Sites publication [10] which was then further extended by the author leading to the data used as the basis of Yuan Gui's master thesis [94] (specifically, the author provided the analysis discussed in Section 4.1.2). The performance improvements and new scoring function were implemented and tested by Catalin Patulea, however this was done using the PIPE2 implementation as a starting point. To fully utilize all of these new features, the author carefully combined them all into the latest version of PIPE, MP-PIPE2.

\subsection{PIPE-Sites}

While there are many methods for predicting PPIs, there are far fewer that can determine the specific site of interaction on each protein. PIPE-Sites is an extension to the original PIPE algorithm used to predict the interaction site on both proteins involved in an interaction. This section will describe the algorithm, how it was evaluated and the study which extended this work (in which the author of this thesis collaborated) 
[10] as well as further work contributing to the thesis of Yuan Gui [94].

\subsubsection{PIPE-Sites Algorithm, Initial Testing and Initial Results}

This section describes the Masters thesis of Adam Amos-Binks [9] in which he developed and tested the PIPE-Sites algorithm. This section will detail the algorithm and briefly describe how it was tested as well as some highlights of a subsequent study reported in [10].

\section{PIPE-Sites Algorithm}

The PIPE-Sites algorithm attempts to identify the interaction site on each protein in a pair of interacting proteins. To do this, the unfiltered PIPE output matrix as described in Section 3.1.1 is examined and significant peaks are identified. Once these peaks have been appropriately outlined, the resulting area within the output matrix is predicted to be the interaction site. The algorithm used here is called the walk algorithm. The input to this algorithm is a PIPE output matrix, and a number $n$ of sites you would like to identify. The output of the algorithm is a list of at most $n$ predicted interaction sites (sometimes there are not $n$ sites that can be considered interaction sites). Typically $n=3$. The walk algorithm works as follows.

- Step 1: First the cell with the largest value in the entire matrix is identified. This will be the top of the current peak under consideration. If this peak's height falls below a given threshold, it is rejected and the algorithm returns as there are no peaks high enough to be considered a potential interaction site.

- Step 2: To ensure that the current peak has a large enough base to be considered an interaction site, the algorithm walks from the peak in four directions (up, right, down, left), noting the height at each step.

- Step 3: Once the height falls below a certain threshold (a percentage of the peak's overall height), the algorithm stops walking in that direction.

- Step 4: Once the algorithm has stopped walking in all four directions, a rectangular area in the matrix representing a predicted interaction site is left. The site is rejected if a minimum number of steps were not taken, as peaks with very small bases are rejected as noise. 
- Step 5: Ignoring the current peak, the algorithm returns to step 1 to identify the next highest peak. This is done at most $n$ times.

An example of this algorithm in action with various height thresholds can be seen in Figure 4.1 and an example of the predicted interaction sites made by PIPE-Sites on a PIPE output matrix can be seen in Figure 4.2.

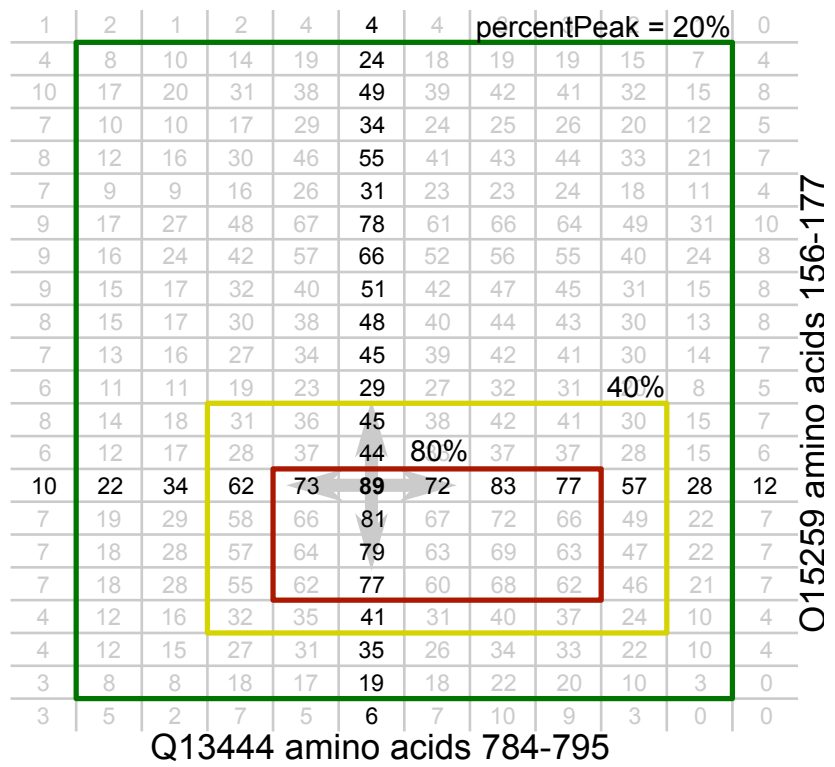

Figure 4.1: Example of the PIPE-Sites walk algorithm identifying a peak and then outlining the predicted interaction site, based on different height thresholds (taken from $[10])$.

\section{PIPE-Sites Evaluation}

To properly evaluate the performance of PIPE-Sites, data from DOMINO (DomainPeptide Interaction Database) [33] was obtained. This data consisted of 265 twosided, lab-confirmed interaction sites of PPIs from S. cerevisiae, and 423 two-sided, lab-confirmed interaction sites for PPIs from H. sapiens. Using these lab confirmed interaction sites, PIPE-Sites could be run on the corresponding PIPE output matrices and its output could be directly compared to known interaction sites. Any interaction site (either predicted or lab confirmed) can be viewed as a rectangle on the PIPE output matrix. To measure how closely the PIPE-Sites identified ranges were to the lab confirmed interaction sites, a distance measure (DM) was developed. This metric 
YCR088W YDR388W 3/4
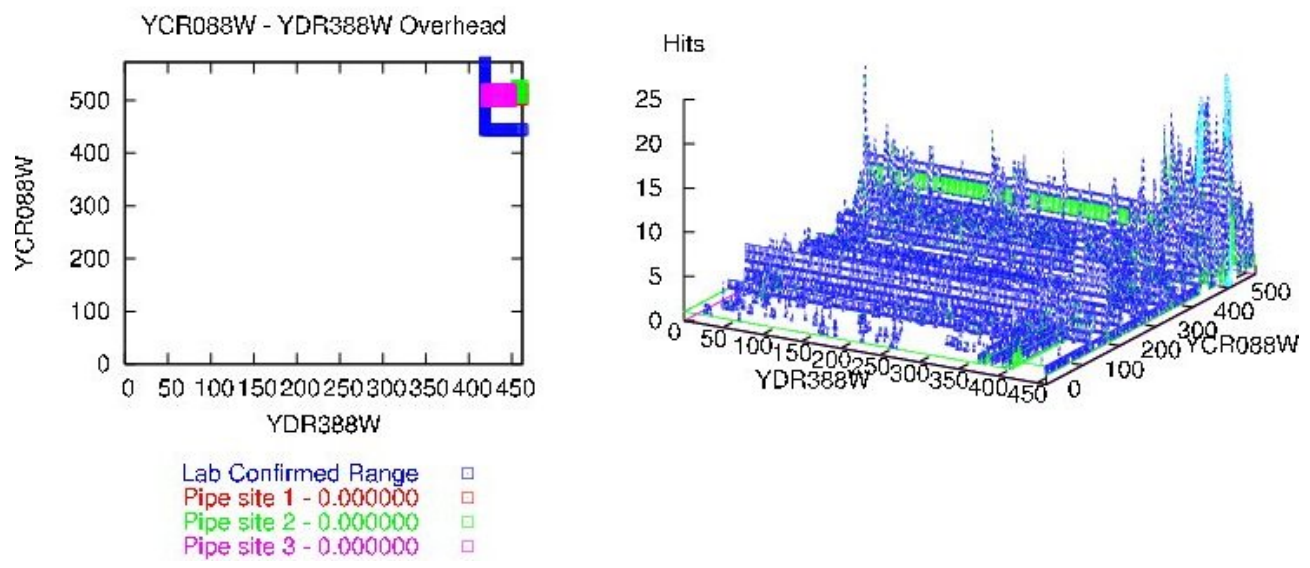

Figure 4.2: Example of interaction sites predicted by PIPE-Sites. On the left is an overhead view of the output matrix showing the confirmed interaction site (blue) along with the PIPE-Sites predicted interaction sites (red, green and purple). On the right is the 3D landscape view of the PIPE output matrix (taken from [10]).

would look at a lab confirmed site compared to a predicted interaction site and give a score between 0 (perfect match) or 1 (interaction sites cover opposite ends of the proteins with no overlap). For two proteins $A$ and $B$, let their lengths be denoted by $A_{l e n}$ and $B_{l e n}$, respectively. Let start $A_{P S}$, end $A_{P S}$, start $B_{P S}$, end $B_{P S}$ denote the location of the interaction sites predicted by PIPE-Sites and start $A_{l a b}$, end $A_{l a b}$, start $B_{l a b}$ and $e n d B_{l a b}$ denote the location of the lab confirmed interaction site.

First, the distances between the interaction sites along each protein is calculated by taking the maximum of the difference between the start positions and the difference between the end positions. If the predicted site is fully contained within the lab confirmed site, this is considered a perfect match and set to 0 . This is because there is a tendency for the lab confirmed sites to be over-estimated due to the laboratory methods used to make these measurements. These distances are calculated for each protein $(\Delta A, \Delta B)$, scaled by the length of their respective proteins. The final DM is the vector addition of the two distances, divided by $\sqrt{2}$ which ensures that the final DM score is between 0 and 1 . The three formulas are given below. 


$$
\begin{aligned}
\Delta A & =\frac{\max \left(\operatorname{start} A_{\text {lab }}-\operatorname{start} A_{P S}, \text { end } A_{P} S-e n d A_{\text {lab }}, 0\right)}{A_{\text {len }}} \\
\Delta B & =\frac{\max \left(\operatorname{start} B_{\text {lab }}-\operatorname{start} B_{P S}, \text { end } B_{P} S-e n d B_{\text {lab }}, 0\right)}{B_{\text {len }}} \\
D M & =\frac{\sqrt{\Delta A^{2}+\Delta B^{2}}}{\sqrt{2}}
\end{aligned}
$$

PIPE was then run on the protein pairs in the data obtained from DOMINO. Those pairs containing peaks higher than 10 were kept, leaving 176 S. cerevisiae and 363 H. sapiens pairs. PIPE-Sites was then run on the unfiltered output matrices of these retained pairs, selecting the top three sites for each pair. For all of the protein pairs run, the DM was calculated for each of the three sites returned by PIPE-Sites with respect to the lab confirmed interaction site. As a baseline measure, three random interaction sites were also chosen for each protein pair and their DM scores were also calculated. When looking at only the highest scoring PIPE-Sites peak, the number of top sites within 10\% of the lab confirmed site (accurate prediction) is six times greater than what would be expected by random chance and the number of top sites more than $50 \%$ away from the lab confirmed site (inaccurate prediction) is about $80 \%$ less than what would be expected by random chance. When looking at two or more PIPE-Sites peaks or randomly generated interaction sites, the minimum DM of the sites is the score given. A summary of the average DM using one, two and three PIPE-Sites peaks compared to the same number of randomly predicted interaction sites is given in Table 4.1 .

PIPE-Sites was also compared with two other methods. These methods do not directly predict the actual interaction sites but rather predict domain-domain interactions as no other directly comparable methods could be found. To do these comparisons, every pairwise combination of domains from each query protein is considered and the amino acid ranges for one or several the highest ranking domain pairs is extracted. DPEA [236] uses known PPIs from a variety of organisms to infer domain-domain interactions and DOMINE [232] combines domain-domain interaction data from two methods based on observed physical association in experimental PDB structures and 13 computational methods, including DPEA. These methods are not applicable to all of the protein pairs that PIPE-Sites was applied to, however 


\begin{tabular}{cccc} 
Peaks used & $\begin{array}{c}\text { Avg. DM in } \\
\text { S. cerevisiae }\end{array}$ & $\begin{array}{c}\text { Avg. DM in } \\
\text { H. sapiens }\end{array}$ & $\begin{array}{c}\text { Avg. DM for } \\
\text { random predictions }\end{array}$ \\
\hline & & & \\
1 & 0.218 & 0.246 & 0.402 \\
3 & 0.168 & 0.213 & 0.320 \\
3 & 0.188 & 0.151 & 0.269 \\
\hline
\end{tabular}

Table 4.1: PIPE-Sites interaction site prediction errors (average DM) for S. cerevisiae pairs, H. sapiens pairs compared to random interaction site predictions. DMs presented here are averaged over all pairs within the given organism (taken from [10]).

all three methods were run on all possible pairs from the DOMINO data and the results are summarized in Table 4.2. As can be seen in this table, PIPE-Sites not only outperforms the other methods but is also more widely applicable.

\begin{tabular}{lcccc}
\hline Organism & $\begin{array}{c}\text { Pairs in original } \\
\text { validation set }\end{array}$ & Method & $\begin{array}{c}\text { Applicable } \\
\text { Pairs }\end{array}$ & $\begin{array}{c}\text { Error rate } \\
\text { (Avg. DM) }\end{array}$ \\
\hline S. cerevisiae & 265 & DPEA & 32 & 0.718 \\
S. cerevisiae & 265 & DOMINE & 144 & 0.363 \\
S. cerevisiae & 265 & PIPE-Sites & 174 & 0.218 \\
H. sapiens & 423 & DPEA & 2 & N/A* \\
H. sapiens & 423 & DOMINE & 266 & 0.306 \\
H. sapiens & 423 & PIPE-Sites & 363 & 0.246 \\
\hline
\end{tabular}

Table 4.2: Interaction site prediction errors (average DM) for PIPE-SItes compared to two other interaction site prediction methods, DPEA [236] and DOMINE [232]. *Too few pairs were applicable to give a meaningful error rate. (taken from [10]).

To evaluate the biological significance of the predicted interaction sites, the InterPro tool and database $[115,184]$ were used to annotate the proteins in the DOMINO data sets and all known domains within these proteins were extracted. A number of 
the identified interaction sites were shown to correspond to domains known to mediate PPIs in both $S$. cerevisiae and H. sapiens proteins. Of the top-ranked PIPE-Sites from the DOMINO data, about half of them were reported by InterPro to correspond to a known domain. This shows that predicted PIPE-Sites correspond to motifs known to be involved in mediating PPIs but can also produce novel domain-domain pairs that could potentially be responsible for mediating PPIs.

\subsubsection{Experimental Validation of Novel PIPE-Sites Predictions}

The study briefly described previously [10] successfully showed that PIPE-Sites is able to predict motifs which correspond to domains known to mediate PPIs. It also describes how novel motifs can be identified which could potentially be responsible for PPIs as well, however offers no experimental proof. This section briefly describes how this work was extended, leading to experimental validation of novel PIPE-Sites, described in [94].

To find suitable novel motifs potentially responsible for mediating PPIs, the top 1,000 S. cerevisiae protein pairs predicted to interact (based on their PIPE scores) were used, as $S$. cerevisiae is a convenient organism to carry out the suitable wet-lab experiments needed to validate the novel motif pairs. The output matrices of the top 1,000 scoring yeast protein pairs were run through PIPE-Sites to try and identify three potential interaction sites per protein pair. Each pair successfully returned three interaction sites, leaving 3,000 predicted interaction sites in total. Using these sites, the pairs of amino acid substrings from each protein making up each predicted interaction site were extracted. Every unique sequence was given a name and, along with its pair, is considered a novel motif pair potentially responsible for a PPI. After giving the motifs names (and thereby eliminating duplicated motifs that occurred in multiple motif pairs), 2,439 unique motifs making up a total of 2,965 unique motif pairs remained. The 402 yeast proteins making up the 1000 predicted PPIs containing the identified motifs were then run through InterPro and any motif overlapping with a known domain was no longer considered novel. This filtering left two groups of novel motif-pairs, those consisting of two novel motifs and those containing exactly one novel motif. There were 1,138 novel motif pairs consisting of exactly one novel motif 
and 272 novel motif pairs consisting of two novel motifs. To make these lists of novelknown and novel-novel protein motif pairs more amenable to wet-lab confirmation, further filtering needed to be done. The motifs needed to be relatively short (under 50 amino acids in length) and they needed to occur relatively close to the end of their respective proteins (within 100 amino acids from either end of their respective proteins). The reason for this was to ease in the extraction of these novel motifs from the natural proteins for experimental validation. Large motifs or motifs occurring closer to the middle of the proteins would make this process more difficult. Once this filtering was completed, it left 35 novel-known motif pairs and 6 novel-novel motif pairs that met all of the established criteria. At this point the data was handed over to Yuan Gui who then further analyzed this data and successfully showed that some of these novel motif pairs actually mediated interactions. He showed this in the following manner. First he found a pair of proteins that did not interact and carried out the appropriate wet-lab experiment to show this. He then extracted the predicted PPI-mediating motifs as discussed above (them being relatively short and near the end of their respective proteins eased this process). He then fused these motifs to the end of the proteins previously shown not to interact (one to each of the proteins). Lastly he carried out an experiment to then show that these altered proteins did in fact now interact. For more details on the experimental setup and further biological analysis see [94].

\subsection{Further Computational Acceleration \& Modified Score Function}

This section will describe further advancements and extensions of the overall PIPE project, namely the introduction of a new scoring metric to evaluate PPIs and a relatively simple yet incredibly powerful code optimization what lead to significant performance improvements. These are two highlights taken from Catalin Patulea's Master's thesis [212] that are especially relevant to work described later in this thesis.

\subsubsection{PIPE Algorithm Modification, Data Structure Optimization \& Performance Improvements}

As previously described in Section 3.2.1, the process of computing the similarity score between window fragments within the core PIPE algorithm was removed and all of 
these computations were pre-processed. This process created a similarity file for each protein which are stored on disk. This similarity file stored, for each sliding window location along the protein in question, a list of proteins that contain a window similar to the current window in question. This altered how the main PIPE algorithm (as described in Section 3.1.1, alterations described in Section 3.2.1) operated while still producing the same output. Before this alteration, the process of calculating the similarity score between amino acid sequences dominated the overall running time of the PIPE algorithm. Since all of this work was now pre-computed and stored to disk in similarity files for each protein, the newer version of the algorithm was dominated by traversing the lists of proteins within these similarity files (steps 3 and 4 of the algorithm described in Section 3.2.1). It was first identified that a slight reordering of the loops within the PIPE algorithm was needed. The algorithm was altered to do the following.

- Steps 0, 1, 2, 3: These steps remain unchanged from the previous version.

- Step 4: For each sliding window location on protein $B$ a list $\left\{B^{\prime}\right\}$ of "similar" proteins is retrieved from the similarity file previously loaded. Next, simply run through each protein in $\left\{B^{*}\right\}$ and see if it is in $\left\{B^{\prime}\right\}$. If it is, the output matrix is incremented at the position representing the current window locations on proteins $A$ and $B$.

- Step 5: The output matrix is analyzed as before to determine in proteins $A$ and $B$ are predicted to interact.

As before, this algorithm is equivalent to the version used in PIPE2 (as described in Section 3.2.1) which itself is equivalent to the original PIPE algorithm (described in Section 3.1.1). The main difference in the two versions of the algorithm is the order of that the loops are processed. By making this alteration, step 4 basically becomes the task of determining if the intersection of the sets $\left\{B^{*}\right\}$ and $\left\{B^{\prime}\right\}$ is nonempty. This is a relatively basic set operation, however the way it was implemented in PIPE2 was inefficient. The similarity files were stored in memory as a linked list of linked lists (where the file was stored in memory as a linked list of window positions, each window position being a liked list of proteins containing a window similar to the respective 
window on the protein in question). This meant that to determine if any interacting protein from $\left\{B^{*}\right\}$ was in the $\left\{B^{\prime}\right\}$, the entire linked list pertaining to $\left\{B^{\prime}\right\}$ would have to be traversed up to $\left|\left\{B^{*}\right\}\right|$ times (once for each protein in $\left\{B^{*}\right\}$ ). It was identified that step 4 in particular could be substantially optimized at the cost of more memory consumption. The basic idea is as follows. First consider every protein in the organism that is currently being processed as an integer, 0 to total number of protein minus 1 (num_proteins). This then gives each protein a unique integer identifier. Instead of loading protein $B$ 's similarity file into a linked list of linked lists, the following was done. For each sliding window location, a set of num_proteins bits was initialized to contain all zeros. For each protein containing a similar window to the current window on protein $B$, the position in relation to its unique integer ID was set to 1 . A set of bits num_proteins long which is all 0s, except in those positions which represent a protein in $\left\{B^{\prime}\right\}$ (which are set to 1 ) is now available. Now, for each protein $B^{*}$ in $\left\{B^{*}\right\}$ an all-zero set of num_proteins bits, except for the position which represents $B^{*}$ is created. This can be done in $\mathrm{O}(1)$ time. The "and" operation is then applied to these two bit sets. If the resulting set of bits (interpreted as an integer) is not zero, then this means that $B^{*}$ is in $\left\{B^{\prime}\right\}$. This relatively straightforward reordering of the loops in the PIPE algorithm and the introduction of the bit set representation of the similarity files means that the possibly expensive task of determining if a given protein was in a large linked list representing a window position on protein $B$ has now been reduced to an $\mathrm{O}(1)$ time operation. Since this operation previously dominated the computation time for PIPE2 and MP-PIPE, the described optimization had a very significant impact on performance.

The algorithm improvements were benchmarked in a variety of ways but, overall, these algorithm modifications and data structure optimizations saw an 8.9x speedup on average for a test set of $S$. cerevisiae protein pairs and an average 14.5x speedup on a test set of $H$. sapiens protein pairs (with up to an $80 \mathrm{x}$ speedup on individual pairs). Generally speaking, the more complex the protein pair is to compute (ie. the larger the sets $\left\{A^{\prime}\right\},\left\{B^{\prime}\right\}$ and $\left\{B^{*}\right\}$ described above get, which directly relate to how many times the inner loops of the PIPE algorithm have to run), the greater the performance gains realized by these modifications. Using this, PIPE could make individual predictions on nearly all protein pairs in a fraction of a second, which leads 
to the possibility of producing proteome-wide, all-to-all predictions for any organisms for which the requisite data is available in a matter of days as opposed to weeks or months. For more details on the algorithm modifications or benchmarking, see [212].

\subsubsection{Modified Score Function}

After successfully improving PIPE's computational performance, Patulea attempted to then reduce PIPE's false positive rate and thus improve the quality of the positive predictions made. Patulea hypothesized that frequently reoccurring sequence windows within proteins may contribute to noise in the resulting PIPE output matrix, which then artificially inflates the resulting PIPE score leading to false positives. To address this issue, Patulea proposed that the score entered into the PIPE output matrix should be normalized by the uniqueness of the window sized sequences currently under consideration. Specifically, instead of simply assigning the total number of hits encountered when comparing the sliding windows of the query proteins to the proteins in the known interaction graph, the ratio of the number of hits found to the total possible number of pairs between proteins similar to the query proteins at the current window locations was used. For example, consider the following hypothetical case of query proteins $A$ and $B$. At window locations $i$ and $j$ respectively, there are lists of proteins containing similar regions, $\left\{A^{\prime}\right\}$ and $\left\{B^{\prime}\right\}$ respectively. At these positions, $\left|\left\{A^{\prime}\right\}\right|=4$ and $\left|\left\{B^{\prime}\right\}\right|=3$ and these two sets of proteins have 6 total known interactions between them. Traditionally this scenario would score a 6 in the output matrix but, according to this new scoring method, the output matrix would be assigned $6 / 12=0.5$ in cell $(i, j)$. Now, consider the case at a different location where the window sequences happened to be more highly reoccurring but did not show any evidence to mediate PPIs. For example, in this new location $\left(i^{\prime}, j^{\prime}\right)$, $\left|\left\{A^{\prime}\right\}\right|=7$ and $\left|\left\{B^{\prime}\right\}\right|=5$ but still had a total of 6 known interactions between the now 35 total possible number of pairs of proteins from $\left\{A^{\prime}\right\}$ and $\left\{B^{\prime}\right\}$. Intuitively one would think that these regions are less likely to be involved in mediating PPIs than the previous example, as there are fewer examples of links between the two similarity lists. However the conventional scoring method would still assign output matrix cell $\left(i^{\prime}, j^{\prime}\right)$ a score of 6 . The new modified score function addresses this by assigning cell $\left(i^{\prime}, j^{\prime}\right)$ a score of $6 / 35=0.17$. This example is illustrated in Figure 4.3. The value 
of the final score is taken as the average cell value across the entire output matrix and if this value is above a tunable threshold, the query protein pair is predicted to interact.
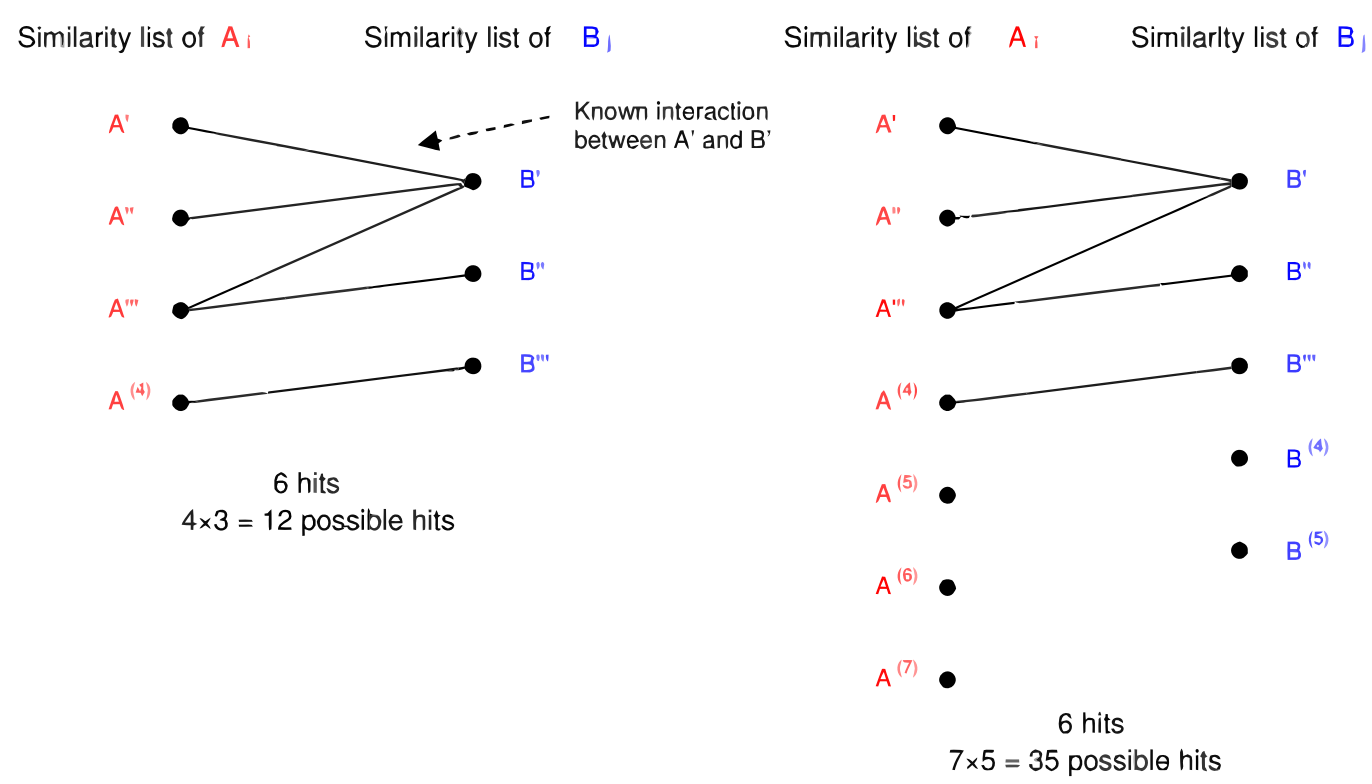

Original score: 6

Original score: 6

$\begin{aligned} & \begin{array}{l}\text { Accounting for } \\ \text { uniqueness: }\end{array} \\ & 12\end{aligned}=0.50$

Accounting for
uniqueness:

(a)

(b)

Figure 4.3: Comparison between the calculation of the traditional PIPE score and the similarity-weighted PIPE score. The similarity-weighted PIPE score takes into account how unique a given pair of amino acid motifs are when calculating their perceived influence on PPIs (taken from [212]).

LOOCV tests similar to those described in Section 3.2.3 were done, however with one major adjustment. Instead of the LOOCV tests described previously (when attempting to predict whether the known-interacting protein pair $(A, B)$, one simply removes $(A, B)$ from the knowledge base), in these tests all protein pairs containing either protein $A$ or protein $B$ are removed from the knowledge base before running a prediction on pair $(A, B)$. This is the most extreme form of LOOCV tests, which would lead to an extremely conservative estimate of performance. That said, LOOCV tests of this manner were carried out on both $S$. cerevisiae and $H$. sapiens data using 
both the traditional PIPE score and the new weighted score as described here. Using the traditional operating point with a specificity of $99.95 \%$, the traditional PIPE score achieved sensitivities of $2.17 \%$ and $1.26 \%$, respectively. However, using the new weighted score, PIPE achieved sensitivities of $3.05 \%$ and $16.19 \%$ respectively, a marked increase over the traditional score. For more details on this weighted scoring measure or more details on the LOOCV tests performed, see [212].

\section{$4.3 \quad$ MP-PIPE2}

Although both PIPE-Sites (described in Section 4.1) and the performance improvements and new weighted scoring function introduced by Patulea (described in 4.2) were great improvements on the previous versions of PIPE, they were not applicable to proteome-wide, all-to-all predictions within a given organism since they were all built upon PIPE2 (described in Section 3.2) and thus were susceptible to the same downfalls of PIPE2 (described in Section 3.3). Because of this, all of these advancements needed to be combined into a new version of PIPE, MP-PIPE2. To this end, MP-PIPE (designed to overcome the inherent downfalls of PIPE2, described in Section 3.3) was used as a starting point. The PIPE-Sites functionality was added to allow for PPI site prediction to be done on all protein pairs, regardless of whether they were predicted to interact of not. This would then allow anyone who wanted to lower the accept threshold at a later date to still be able to extract PPI site data without rerunning PIPE. The algorithm modifications and optimized data structures described by Patulea were also incorporated to allow for the described performance improvements to be witnessed on a massive scale. The new weighted function was also added to the code. This meant that the code had to maintain two separate output matrices (one for each score function), which naturally impacted on the memory usage. However, the value added in getting both scores (allowing the user to use whichever score and accept threshold they want) meant that incorporating the new score function while maintaining the old one was worthwhile. MP-PIPE's ability to scale down slightly if needed (by simply running fewer threads if there is insufficient memory space) meant that incorporating all of these new aspects into one version of PIPE was not only possible but also the most logical.

MP-PIPE2 is the current version of PIPE being used and was used on all projects 
described later in this thesis. Although no publication has been made describing this new version of PIPE, the fact that all valuable improvements and extensions to PIPE have been combined into a single, convenient to use package able to scale from a single desktop PC to a massive compute cluster is in and of itself notable. 


\section{Chapter 5}

\section{Applications of PIPE \& Analysis of Resulting Data}

This chapter will summarize three projects which built off of MP-PIPE produced data. The projects will be presented in chronological order based on their publication date. The first section details the study of using MP-PIPE to produce cross-species predictions. In this case, interactions from one species were used to predict interactions in another species as well as using data from a collection of species to predict interactions within a single species. The second section describes work based off of a revised $S$. cerevisiae proteome-wide prediction, in which novel interactions within the prediction results were analyzed. It was hypothesized that some of these previously uncharacterized proteins were involved with non-homologous end-joining in response to DNA damage within a cell. This hypothesis was shown to be true through wet-lab experiments. The third section presents an in-depth analysis of the predicted H. sapiens interactome (as described in Section 3.3.3) to show the variety of ways to extract useful biological information out of novel MP-PIPE data. Although these projects were collaborative in nature (some more focused on biological results as opposed to the computational), the author of this thesis was central to the computational work done.

\subsection{Cross-species PPI prediction using MP-PIPE}

This section will detail the application of MP-PIPE to organisms previously not analyzed by PIPE as well as the ability of MP-PIPE to use the interaction data from one organism to predict interactions in another. On top of this, the ability to use a compilation of interactions from a set of different organisms to predict interactions within a given organism was tested. MP-PIPE was used in this project as opposed to MP-PIPE2 since MP-PIPE2 was still in development at the time. This work can be found in the following publication [225]. 


\subsubsection{Application of MP-PIPE to a variety of organisms}

This study focused on the following organisms: Escherichia coli, Schizosaccharomyces pombe, Saccharomyces cerevisiae, Caenorhabditis elegans and Homo sapiens. Since E. coli and $S$. pombe were relatively easy to process, entire proteome-wide, all-to-all predictions were made with MP-PIPE. An updated list of proteins and known PPIs were collected for $S$. cerevisiae and an updated predicted interactome was generated by MP-PIPE. The previous $C$. elegans and $H$ sapiens prediction results were also used. Table 5.1 summarizes the available data at the time the study was done.

\section{Organism \# of Proteins \# of Known Interactions Interaction Database}

\begin{tabular}{lccl}
\hline C. elegans & 23,684 & 6,607 & BioGRID [37] \\
E. coli & 4,290 & 16,235 & EcID [11] \\
H. sapiens & 22,513 & 41,678 & HPRD[133] \& \\
& & & BioGRID [37] \\
S. cerevisiae & 6,716 & 43,591 & BioGRID [37] \\
S. pombe & 5,024 & 2,951 & BioGRID [37] \\
& & & \\
\hline
\end{tabular}

Table 5.1: Summary of data gathered for organisms processed by MP-PIPE.

Leave-one-out cross-validation tests, as described in 3.2.3, were carried out on all organisms discussed above. As was done previously, an operating point with an extremely high specificity was chosen for each organism to ensure an extremely low false positive rate. The usual specificity of $99.95 \%$ was not used in the case of $E$. coli here as the sensitivity at that operation point was zero. Because of this, the operating point for E. coli was lowered to a specificity of $99.0 \%$. The sensitivities for these organisms are summarized in Table 5.2.

Another way to look at the predictive performance of MP-PIPE for a given organism is to examine its performance over the entire range of sensitivities/specificities. One way to do this is to view the data on a receiver operating characteristic (ROC) curve. These plots have the false positive rate (1 - specificity) on the $x$-axis and the true positive rate (sensitivity) on the $y$-axis. Each point on the plotted line can act 


\begin{tabular}{lll} 
Organism & Specificity (\%) & Sensitivity (\%) \\
& & \\
\hline C. elegans & 99.95 & 9.97 \\
E. coli & 99.0 & 14.48 \\
H. sapiens & 99.95 & 23.22 \\
S. cerevisiae & 99.95 & 8.77 \\
S. pombe & 99.95 & 16.89 \\
\hline
\end{tabular}

Table 5.2: Summary of predictive performance of MP-PIPE for organisms processed by MP-PIPE.

as an operating point for MP-PIPE and that point defines the sensitivity and specificity for MP-PIPE at that operating point. This is useful as sometimes only a small number of proteins/PPIs are being investigated and, in these cases, an extremely high specificity might not necessarily be needed. Generally speaking, the larger the area under a ROC curve (or, in other words, the closer it bends towards the top left corner) the better MP-PIPE performs on that given organism. The ROC curves for the organisms studied here can be seen in Figure 5.1.

After examining the ROC curves a few things become clear. First of all, MP-PIPE performs the worst on E. coli. To determine how the other organisms compare, it depends at which operating point one looks at. For example, at the usual MP-PIPE operating point of $99.95 \%, H$. sapiens scores the highest sensitivity followed by $S$. pombe, C. elegans and S. cerevisiae. However, each of these organisms outscore $H$. sapiens at other points on the ROC curve. The ROC curves are used to give a general idea of how well MP-PIPE performs overall. Overall, MP-PIPE performed very well on H. sapiens, S. pombe and C. elegans, well on S. cerevisiae and worst (while still producing novel data that is undoubtedly valuable) on $E$. coli. It is interesting to note that E. coli is a prokaryotic organism while all other organisms studied with PIPE are eukaryotic. Prokaryotic and eukaryotic organisms are fundamentally different types of organisms. These differences include cellular structure and organization, genome and gene size, amount of coding vs. non-coding DNA, amount of genetic redundancy as well as how a number of key cellular functions (including DNA replication, protein 


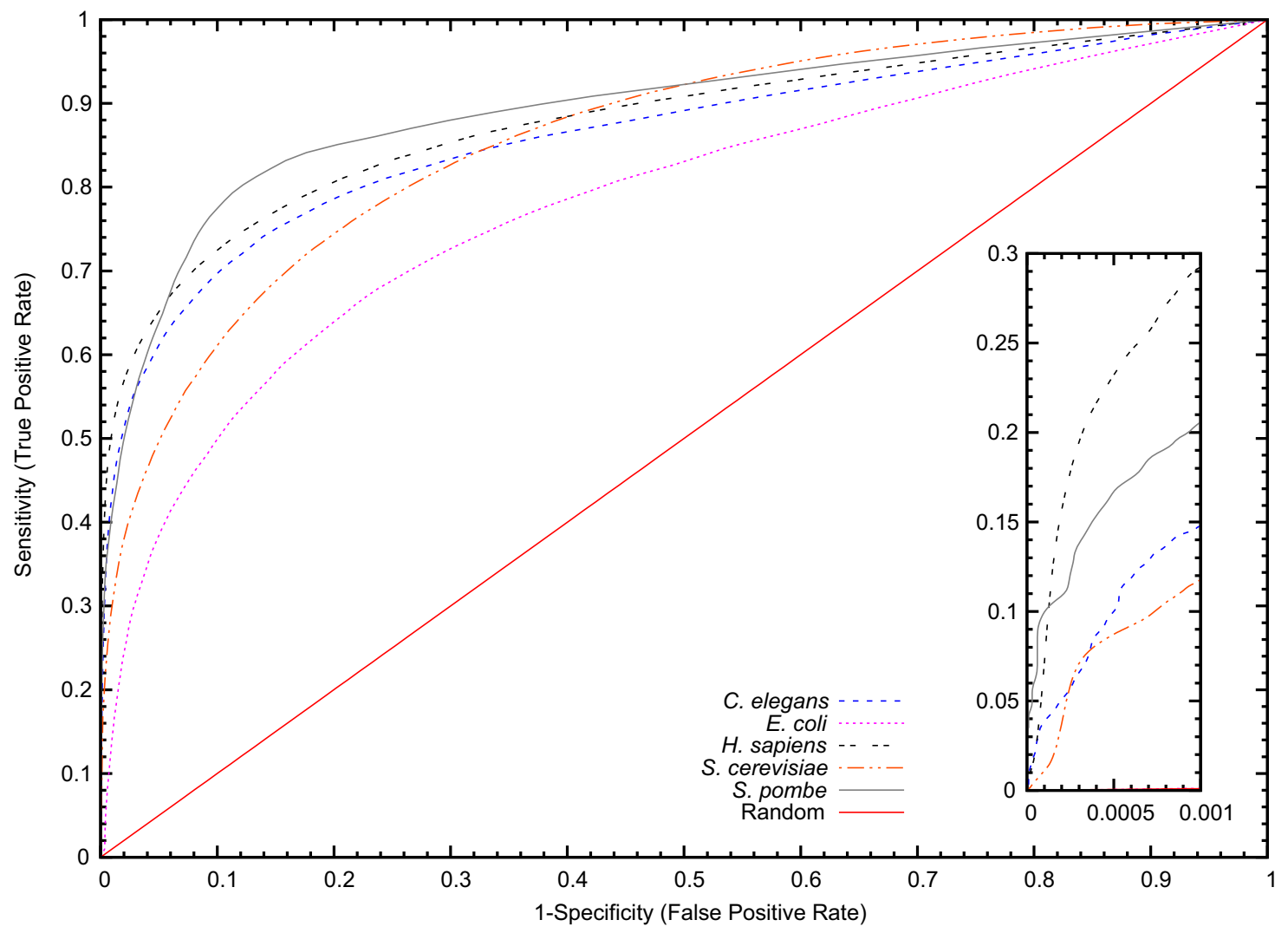

Figure 5.1: ROC curves showing MP-PIPE predictive performance on $C$. elegans, $E$. coli, H. sapiens, S. cerevisiae and $S$. pombe. The solid red line shows the performance of simply choosing randomly. The inset shows MP-PIPE's typical operating range $(\sim 99.95 \%)$ and, due to scaling, the solid red line appears flat (taken from [225]).

synthesis and post-translational modification) are carried out, among others [21, 281]. This result hints at the fact that the core PIPE algorithm may only be suitable to predicting PPIs in eukaryotic organisms and improving PIPE's predictive performance prokaryotic organisms such as E. coli is currently a potential avenue of future research.

\subsubsection{Using MP-PIPE to produce cross-species PPI predictions}

Next MP-PIPE was tested to see if it could use the interaction data from one organism to predict interactions successfully in another organism. To test this, the interaction graph given as an input to PIPE (which would subsequently be preprocessed into PIPE database files as previously described in Section 3.2.1) would be from organism 1 and this would then be used to predict interactions from organism 2. To evaluate 
MP-PIPE's ability to carry out these cross-species predictions, tests similar to the leave-one-out cross-validation tests described in Section 3.2.3 would be carried out. The only difference is that when evaluating if the interactions from organism 1 could be used to predict the known interactions from organism 2, these known interactions wouldn't need to be "left out", as they weren't in the interaction graph to begin with. In this case the organisms $H$. sapiens and $S$. cerevisiae were used simply because they had the highest number of previously reported interactions. Figure 5.2 shows the ROC curves for the four combinations of $H$. sapiens and $S$. cerevisiae data: known $H$. sapiens interactions used to predict $H$. sapiens interactions (HS-HS), known $S$. cerevisiae interactions used to predict $H$. sapiens interactions (SC-HS), known $S$. cerevisiae interactions used to predict $S$. cerevisiae interactions (SC-SC) and known H. sapiens interactions used to predict $S$. cerevisiae interactions (SC-SC).

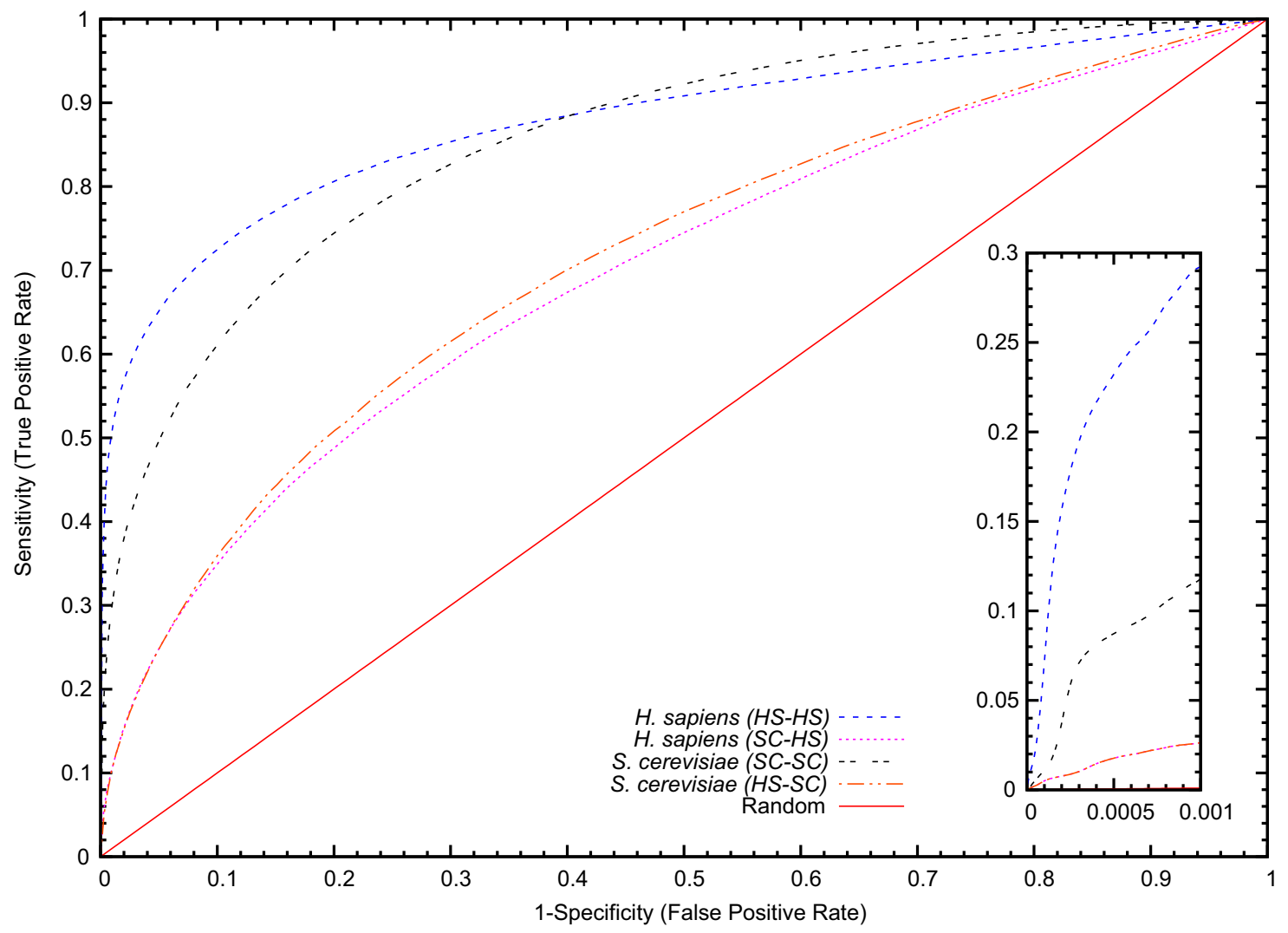

Figure 5.2: ROC curves showing MP-PIPE predictive performance on cross-species PPI prediction, specifically comparing the $H$. sapiens and $S$. cerevisiae ROC curves to the ROC curves of MP-PIPE using H. sapiens interaction to predict $S$. cerevisiae interactions and vice versa (taken from [225]). 
As can be seen in the ROC curves, using interaction data from $H$. sapiens to predict interactions in $H$. sapiens performs better than using $S$. cerevisiae interactions to make these predictions. The same is true (using an organism's own known interactions as the PIPE database) for S. cerevisiae. That said, MP-PIPE can still produce valuable predictions when used to make cross-species predictions as can be seen when comparing these ROC curves to the random decision rule. If one wants to extract some valuable data using this method, the specificity could simply be lowered slightly to achieve a higher sensitivity. In this case MP-PIPE can achieve sensitivities between $46 \%$ and $48 \%$ and a specificity of $80 \%$.

\subsubsection{Using a compilation of interactions from a set of different organisms to make PPI predictions}

Finally, all of the interaction data collected was assembled and used as the reference databse to make predictions within a single organism. LOOCV tests were performed for each organism using this database and the resulting ROC curves can be seen in Figure 5.3.

As can be seen in this figure, MP-PIPE performs reasonably well on all of the eukaryote organisms studied (all organisms other than E. coli). Interestingly, compiling all of the interaction data actually produces better results for $H$. sapiens at a specificity of $80 \%(54-55 \%)$ as opposed to simply using S. cerevisiae interactions as described in the previous section (48\%). These results, coupled with those from the previous section, suggest two things. First, MP-PIPE can successfully use interaction data from one species to make meaningful predictions in a second species. Secondly, the interaction codes underlying the general assumptions the main PIPE algorithm is based upon seem to be somewhat evolutionarily conserved, at least in eukaryotes.

\subsubsection{Independent comparison of PIPE to other methods}

In [210] Park independently evaluated the PIPE method in comparison to three other methods [96, 176, 259]. In this study, four test categories were established: (a) $H$. sapiens data to predict $H$. sapiens PPIs, (b) S. cerevisiae data to predict $S$. cerevisiae PPIs, (c) H. sapiens data to predict S. cerevisiae PPIs and finally (d) S. cerevisiae to predict H. sapiens PPIs. Tests (a) and (b) are designed to show how well each 


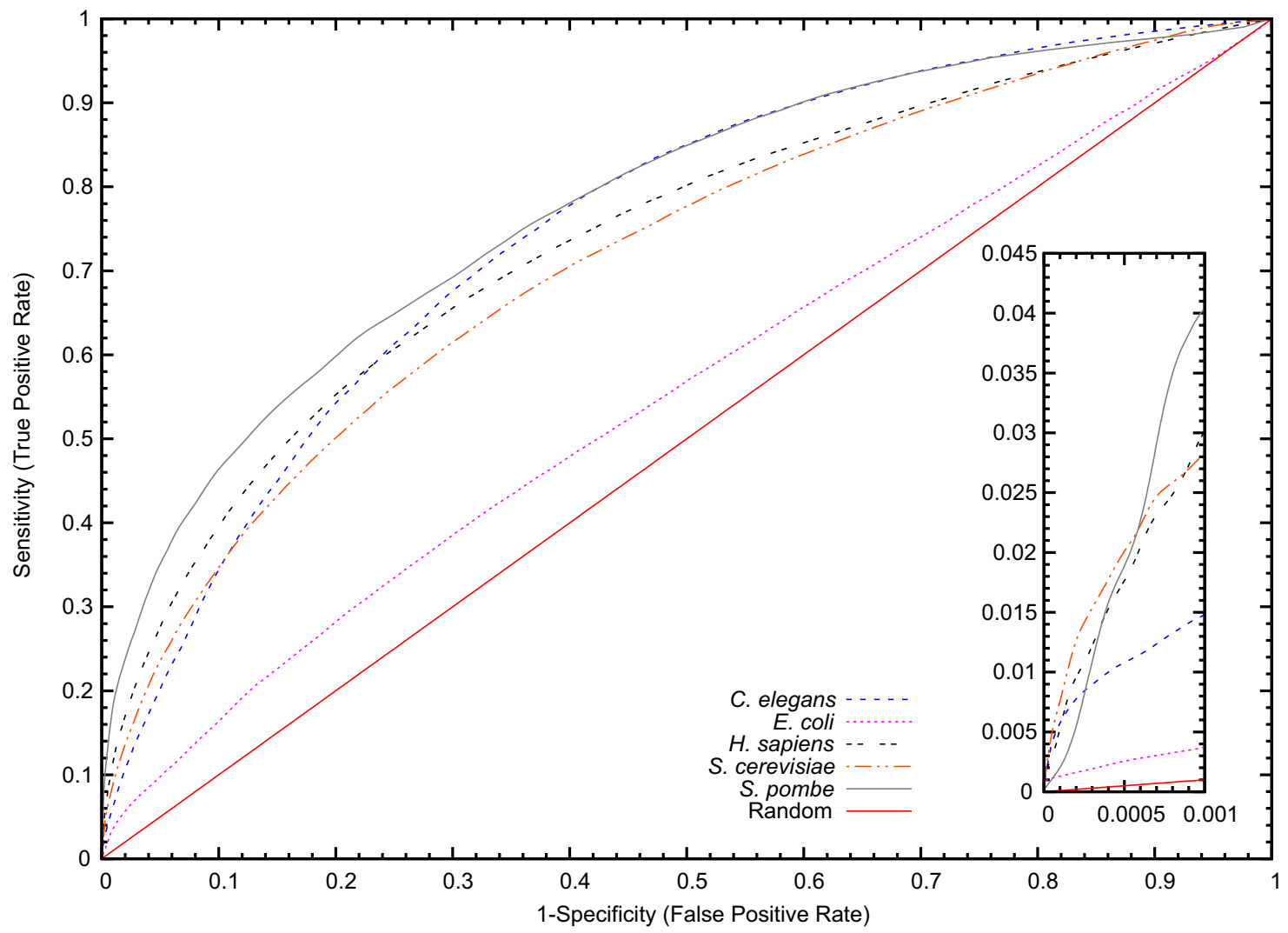

Figure 5.3: ROC curves showing MP-PIPE predictive performance using interaction data from a collection of organisms., specifically using interaction data from C. elegans, E. coli, H. sapiens, S. cerevisiae and S. pombe to make predictions on each organism individually (taken from [225]).

method can use an organism's interaction data to predict its own interactions. Tests (c) and (d) are designed to show how well the methods can perform cross-species predictions. As previously mentioned, a method's ability to perform PPI predictions at a very high specificity is of utmost importance and Figure 5.4 shows a comparison of PIPE and the other three methods for specificities above $95 \%$. As can be seen in this figure, other than a close tie in 5.4.(a), the PIPE method vastly outperforms the others. In fact, at the typically chosen operating point of a specificity of $99.95 \%$, PIPE outperforms the other methods by anywhere from 3.6 to 17.2 times. It should also be noted that in Park's comparison, he simply used the default parameter values and did not preform any of the necessary parameter tuning as described in Section 3.1.1. 
A

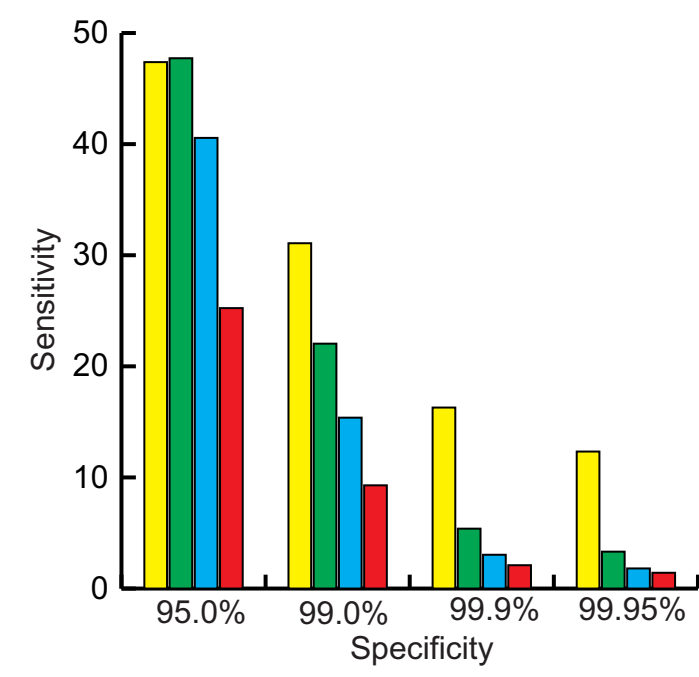

C

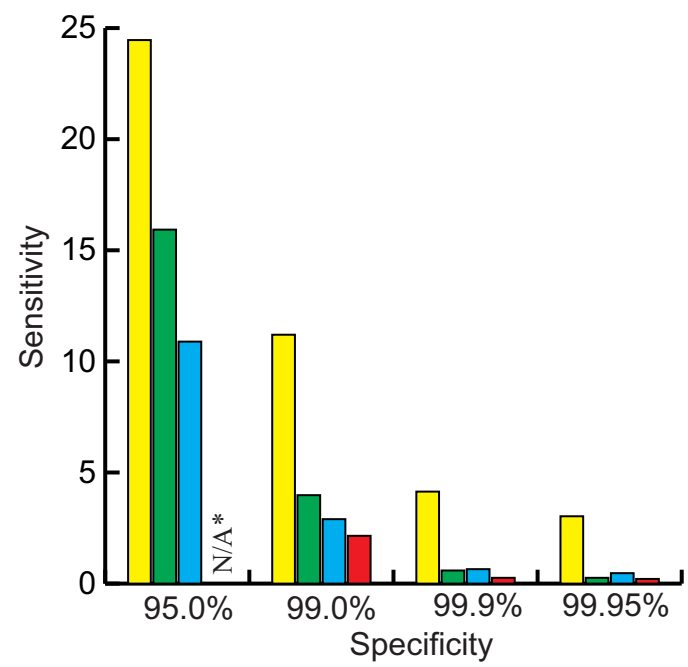

B

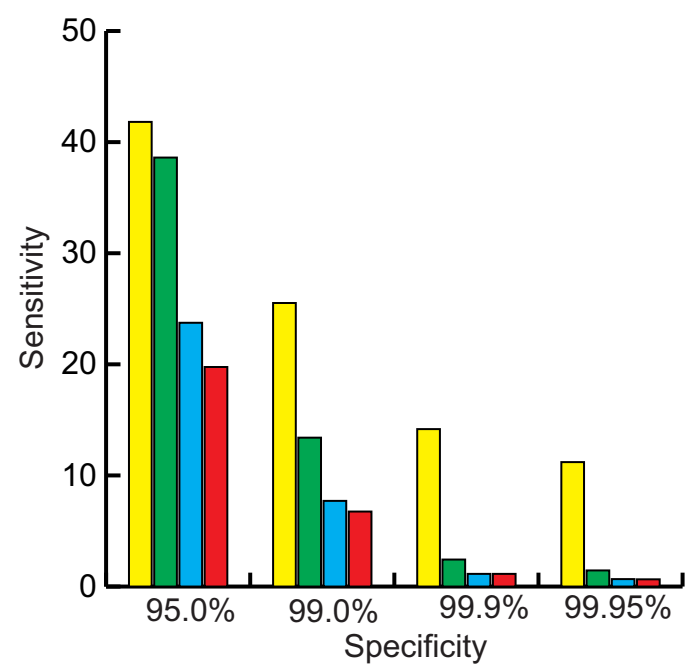

D

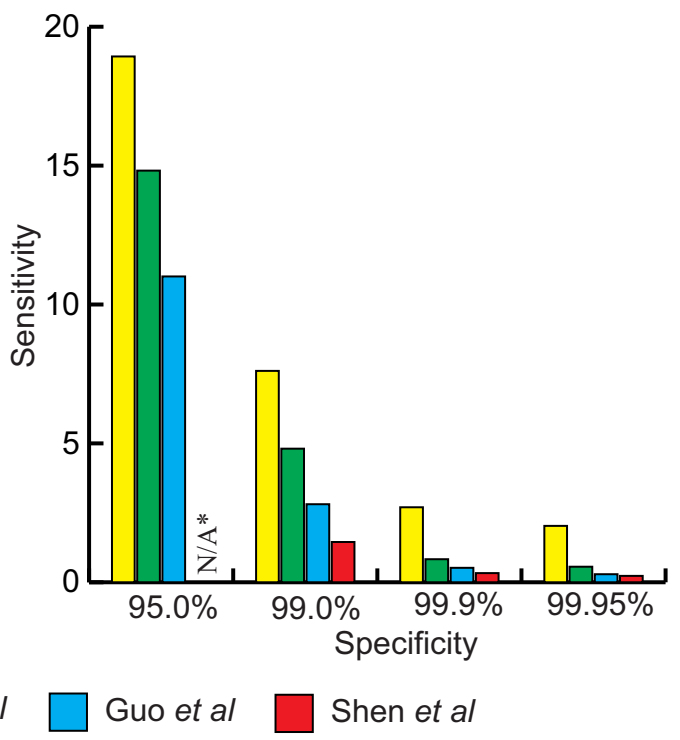

Figure 5.4: Comparing the sensitivities and specificities achieved by PIPE and other methods $[96,176,259]$ as done in $[210]$. (a) Using $H$. sapiens data to predict $H$. sapiens PPIs. (b) Using S. cerevisiae data to predict S. cerevisiae PPIs. (c) Using $H$. sapiens data to predict $S$. cerevisiae PPIs. (d) Using $S$. cerevisiae to predict H. sapiens PPIs. ${ }^{*}$ The experiment by Park in [210] did not have results for $95.0 \%$ specificity for [259] in (c) and (d): the results for this method jumped from 2-3\% specificity to $97-98 \%$ with no intermediate values in both experiments (taken from [225]). 


\subsection{Analysis of $S$. cerevisiae predicted PPI data used to infer novel biological information}

Up until this point, proteome-wide, all-to-all predictions have been made for a handful of organisms accompanied by relatively high-level, superficial biological analysis and a few wet-lab experiments. In this section a study of S. cerevisiae data produced by MPPIPE, in which a more in-depth approach was taken to assign functional information to previously uncharacterized proteins based primarily on MP-PIPE predictions is briefly described. The full study was reported in [202].

The cell cycle is a series of events that a cell naturally goes through before it divides into two identical cells. During this process the cell must pass through a series of internal checkpoints. These checkpoints can be delayed in the case that the cell is not ready to proceed with mitosis. An example of this is when DNA damage occurs. Once a checkpoint has been reached, the cell can, for example, verify the integrity of the DNA and proceed to the next stage of the cell cycle. If DNA damage has occurred, the cell can delay its progression to allow for the DNA to be repaired. Double-stranded DNA breaks (DSBs) are regarded as the most severe form of DNA damage. Non-Homologous End Joining (NHEJ) is the main mechanism to repair DSBs in mammals and is highly conserved between humans and yeast, making investigations into NHEJ in S. cerevisiae particularly interesting [202].

To look further into the mechanics of NHEJ in S. cerevisiae, the updated set of high confidence $S$. cerevisiae PPIs also used in the study descibed in Section 5.1 was futher investigated. Leave-one-out cross-validation tests were done (as described in Section 3.2.3) and an operating point with a specificity of $99.95 \%$ was chosen. The resulting positive predictions were then investigated to identify proteins potentially involved in NHEJ, based on their interactions. Two proteins (Pph3p and Psy2p) not previously thought to be involved in DNA damage repair through NHEJ were predicted to interact with a number of proteins known to be involved in DNA damage repair and proteins involved with cell cycle progression (specifically, they were predicted to interact with the cell cycle checkpoint protein Rad50p). It was shown that the deletion of either PPH3 or PSY2 genes (which encode for proteins Pph3p and Psy2p, respectively) reduced the cell's ability to perform NHEJ. It was also shown that the overexpression of another cell cycle checkpoint protein Chk1p (which works 
in parallel to Rad50p) could compensate for the loss of either Pph3p or Psy2p. With this in mind, it was also shown that the gene deletion of either PPH3 or PSY2 in conjunction with CHK1 (which encodes for Chk1p) further reduced the cell's ability repair damaged DNA. Through another experiment it was shown that Pph3p and Psy2p are not actually involved in the mechanics of NHEJ but rather in the regulation of the process, which was both consistent with previous findings and with the interactions predicted by PIPE. For more details on the experimental setup or more biological analysis, see [202].

\subsection{In Depth Analysis of the Predicted H. sapiens Interactome}

This section describes the subsequent analysis of the proteome-wide, all-to-all $H$. sapiens predictions made by MP-PIPE described in Section 3.3.3. This analysis was carried out in a number of different ways featuring a range of expertise from different collaborators. For more details see [255].

\subsubsection{Adding Credibility to the Novel prediction made by PIPE}

Although stringent leave-one-out cross-validation tests were performed on the H. sapiens predictions, further work was done to add credibility to the predictions made by MP-PIPE. The next several sections describes measures taken to provide this additional credibility.

\section{PIPE predictions compared to new large-scale PPI detection experiments}

To further evaluate PIPE's predictive performance, an independent set of experiments using the Lentivirus-delivered, Gateway-compatible affinity Tagging System coupled with Mass Spectrometry (LGTS-MS) were undertaken. In these experiments, a bait protein is used and exposed to a large sample of other proteins from the organism in question. Once this is done, one can identify the proteins which remain attached to the bait. Four H. sapiens proteins (CBX1 (P83916), RNF2 (Q99496), H2AFX (P16104), and RBBP4 (Q09028)) were used in these experiments. For each of these bait proteins, a set of prey proteins were identified through these LGTS-MS experiments. Care needs to be taken when comparing these results directly to the predicted 
H. sapiens interactions because many of the reported prey may not be interacting directly with the bait in question, but may interact through a one or more intermediary proteins. To combat this problem, the results from the LGTS-MS experiments were compared to the proteins "reachable" to the bait proteins within the predicted $H$. sapiens interacting graph (where reachable is defined as those proteins that interact directly or through one or two intermediary proteins with the bait protein). Overall, the previously reported interactions can only explain an average of $10.89 \%$ of the prey proteins found in these experiments. When the novel predictions made by MP-PIPE are added to this analysis, the prey proteins identified jump nearly 3 times to $29.31 \%$ without drastically increasing the total number of proteins reached from the bait. The results for this comparison can be seen in Table 5.3.

\begin{tabular}{lccc}
\hline Bait & \# of Prey & $\begin{array}{c}\text { Reachable Proteins } \\
\text { (Known/PIPE) }\end{array}$ & $\begin{array}{c}\text { Prey Reached } \\
\text { (Known/PIPE) }\end{array}$ \\
\hline Q09028 & 301 & $112 / 201$ & $56 / 99$ \\
P83916 & 474 & $91 / 244$ & $59 / 178$ \\
P16104 & 209 & $39 / 207$ & $11 / 82$ \\
Q99496 & 292 & $16 / 24$ & $13 / 15$ \\
\hline
\end{tabular}

Table 5.3: Comparison of LGTS-MS experiments in H. sapiens to PIPE predictions. The \# of prey refer to the number of proteins found during the LGTS-MS experiements. The reachable proteins refer to those proteins which can be reached from the bait protein either directly or through one or two intermediary known / PIPE predicted interaction.

\section{PIPE Prediction Specificity when dealing with proteins with high sequence similarity}

Although high protein sequence similarity can be a good indication that the proteins may be functionally similar, this is not always the case. To investigate whether PIPE could correctly predict different interactions for proteins with high levels of sequence similarity which are known to have differences in their activity, two sets of proteins were investigated. The first set of proteins investigated were fibroblast growth factors 
(FGF) which interact with fibroblast growth factor receptors (FGFR). 22 of these proteins were investigated, many of them having no predicted interactions. Five of them, however, did have predicted interactions and they had notable differences in their interaction profiles. These interactions are summarized in Table 5.4.

\begin{tabular}{ll}
\hline FGF & FGFRs \\
\hline & \\
P21781 & P11362* $^{*}$ P22607*, P21802 \\
O95750 & P22455 \\
O15520 & P21802 \\
P09038 & P11362, P22607*, $\mathrm{P} 21802^{*}$ \\
P05230 & P11362, P22607, P21802, P22455 \\
\hline
\end{tabular}

Table 5.4: PIPE predicted interactions between the highly sequence similar fibroblast growth factors (FGF) and their receptors (FGFRs). Of the 22 FGF proteins investigated, only the displayed 5 had predicted interactions. Proteins marked with a* represent novel interactions.

A similar investigation was done on proteins which are known activators of cyclindependent kinases (CDKs) and their predicted regulators. As with the FGF proteins, these proteins have high sequence similarity while being known to interact with different partners. 21 CDK proteins were investigated and 17 had predicted interactions, varying wildly in interaction profile. The predicted interactions for these CDK proteins are summarized in Table 5.5. These results, taken with those above for FGF proteins, show that although PIPE relies solely on protein sequence to make its predictions, it can pick up on relatively slight variations in sequences (that result in functionally different proteins) by predicting different integrating partners, hinting at a specificity that may not be immediately obvious.

\subsubsection{Network-wide analysis of high degree and high betweenness centrality proteins}

Topological features of PPI networks are often of biological interest. The first aspect of the predicted interaction network to be investigated was to look at proteins with high degree (hubs) and proteins with high betweenness centrality. To find the hub 
CDK Regulators of CDK

\begin{tabular}{|c|c|}
\hline Q96Q40 & P63104*, P31946* \\
\hline O94921 & P63104*, P31946 \\
\hline Q9UQ88 & P31946*, P63104* \\
\hline Q00526 & $\begin{array}{l}\text { P31946*, P49918*, P42773*, Q16667, P46527, } \\
\text { P38936, P55273*, Q13319*, P63104*, Q15078* } \\
\text { Q96SZ6*, Q8IWU2*, O15392* }\end{array}$ \\
\hline Q00534 & $\begin{array}{l}\text { O15392*, P42772*, P46527, P49918*, P42773, } \\
\text { P38936, Q8N726, P55273 }\end{array}$ \\
\hline Q00537 & P63104, P31946*, Q15078* \\
\hline Q00536 & P63104, P31946*, Q15078 \\
\hline Q00535 & $\begin{array}{l}\text { O15392*, P38936*, P46527, P31946*, Q13319, } \\
\text { Q96SZ6, Q8IWU2, P63104*, Q15078, Q16667* }\end{array}$ \\
\hline Q9BWU1 & O75448*, P42772*, Q15648*, Q93074*, Q9UHV7*, O75586* \\
\hline Q15131 & P31946*, P63104* \\
\hline P50750 & Q15078 \\
\hline P06493 & $\begin{array}{l}\text { P46527*, Q13319*, P63104*, Q96SZ6* }, \text { P38936, } \\
\text { Q8IWU2*, P31946*, Q16667, O15392, Q15078* }\end{array}$ \\
\hline P11802 & $\begin{array}{l}\text { P38936, P42773, Q8N726, P55273, P42772, } \\
\text { P46527, P49918, O15392, P42771* }\end{array}$ \\
\hline P21127 & P31946, P63104* \\
\hline P24941 & $\begin{array}{l}\text { P38936, P31946*, P63104*, P46527, Q16667, Q8IWU2*, } \\
\text { P49918*, Q15078*, Q96SZ6*, Q13319*, O15392 }\end{array}$ \\
\hline Q07002 & P31946*, P63104*, Q15078* \\
\hline Р49336 & O75586, P42771*, Q93074, Q15648, P42772, Q9UHV7, O75448 \\
\hline
\end{tabular}

Table 5.5: PIPE predicted interactions between the highly sequence similar cyclindependent kinases (CDK) proteins and their regulators. Of the 21 FGF proteins investigated, only the displayed 17 had predicted interactions. Proteins marked with $\mathrm{a}^{*}$ represent novel interactions.

proteins, the proteins were simply sorted by their degree in the predicted interaction network. The betweenness centrality of a protein $V$ within the predicted interaction graph is the ratio of the number of shortest paths between proteins $A$ and $B$ on which $V$ lies and the total number of shortest paths between $A$ and $B$, summed over all possible protein pairs. More formally, 


$$
\begin{aligned}
B C(V) & =\sum_{A \neq V \neq B} \frac{\sigma_{A B}(V)}{\sigma(V)} \\
\text { where, } & \\
\sigma_{A B}(V) & =\# \text { shortest paths from } A \text { to } B \text { through } V \\
\sigma(V) & =\# \text { shortest paths between } A \text { and } B
\end{aligned}
$$

The betweenness centrality was calculated for each protein in the graph and both of these measures were used to find proteins of interest. Protein degree within an interaction network is thought to be a good indicator of that protein's biological importance within the organism $[125,307]$. The top 10, 25 and 50 hubs were looked at in detail and statistical analysis of gene ontology (GO) term enrichment was performed. The proteins in question were found to be enriched for proteins involved with transcription and gene expression (the process through which the information encoded in a gene is translated into a gene product, ie. a protein). This data is summarized in Table 5.6.

\begin{tabular}{lccc}
\hline Biological Process & Top 10 & Top 25 & Top 50 \\
\hline & & & \\
$\begin{array}{l}\text { Transcription regulation } \\
\text { Regulation of gene } \\
\text { expressions }\end{array}$ & $7 / 2.26 \times 10^{-6}$ & $16 / 3.15 \times 10^{-10}$ & $28 / 1.93 \times 10^{-14}$ \\
& & $16 / 1.69 \times 10^{-9}$ & $29 / 2.82 \times 10^{-14}$ \\
\hline
\end{tabular}

Table 5.6: High degree H. sapiens proteins within the predicted interaction network and associated biological processes. For the top 10, 25 and 50 highest degree proteins the number of proteins with stated biological process and associated $p$-value are given.

Proteins with high betweenness centrality on the other hand are thought to play a central role in the communication between large, highly connected subgraphs, or modules, within an interaction network and often form communication bottlenecks [308]. Again, the top 10, 25 and 50 proteins in terms of betweenness centrality were studied and were found to be enriched for proteins involved in intracellular communication (kinase activity and signalling) and proteins for which communication 
plays a large part in their role in the cell (regularion of cell death). The results for this analysis are summarized in Table 5.7.

\begin{tabular}{|c|c|c|c|}
\hline Biological Process & Top 10 & Top 25 & Top 50 \\
\hline Protein kinase activity & $2 / 5.99 \times 10^{-2}$ & $6 / 1.01 \times 10^{-7}$ & $20 / 8.61 \times 10^{-18}$ \\
\hline Regulation of cell death & $3 / 3.96 \times 10^{-5}$ & $11 / 7.60 \times 10^{-9}$ & $191.82 \times 10^{-12}$ \\
\hline Signalling & $4 / 5.49 \times 10^{-5}$ & $13 / 5.90 \times 10^{-9}$ & $33 / 7.45 \times 10^{-12}$ \\
\hline
\end{tabular}

Table 5.7: H. sapiens proteins with high betweenness centrality within the predicted interaction network and associated biological processes. For the top 10, 25 and 50 proteins sorted by betweenness centrality the number of proteins with stated biological process and associated $\mathrm{P}$-value are given.

These types of network centrality measures are sometimes used to predict proteins possibly involved in disease. Some studies suggest hubs are likely involved in disease while others suggest betweenness centrality as a better indicator [39, 62, 78, 88, 204]. The top 500 proteins with respect to their degree and betweenness centrality were investigated to see how many were known to be involved in disease. Comparing the number of disease related proteins found to what would be expected at random, both measures seem to be good indicators for proteins that are involved with disease, with betweenness centrality having a slightly higher correlation. The results for this are summarized in Figure 5.5.

\subsubsection{Identification of potential protein complexes within the predicted H. sapiens interaction network}

Protein complexes can be defined as groups of proteins which interact with one another to perform a certain function or to participate in a specific biological process. Paracliques are dense subgraphs with high degree of inter-connectivity, which can be computationally identified and could identify potentially novel protein complexes. An algorithm used in other biological studies [47, 67, 149] was used to identify paracliques within the predicted $H$. sapiens interaction network. The algorithm works as follows. 


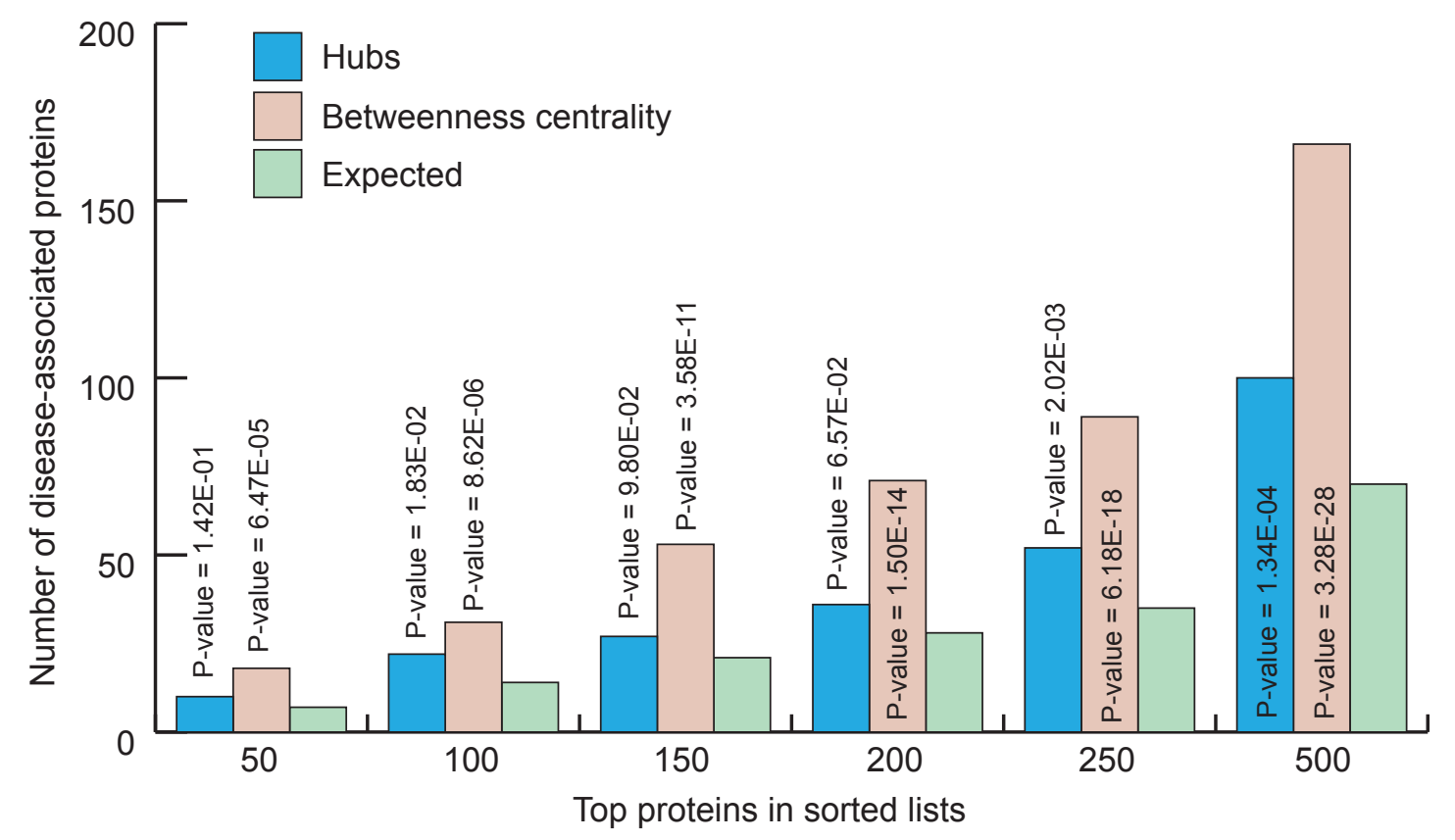

Figure 5.5: The correlation between disease and hubs/high betweenness centrality in the predicted H. sapiens interaction network. Taken from [255].

- Step 1: First, an initial clustering is needed that will be subsequently improved. Any clustering can be used for this initial step, but in this case this clustering was made up of all maximal cliques within the graph. In this context, a maximal clique is a subset of the proteins where every protein is predicted to interact with all of the other proteins in the subset and the size of the subset can not be increased to form a larger clique. This step is performed once while the following steps are run a series of times.

- Step 2: Next, the size of the overlap of all pairs of clusters are calculated and the clusters with the largest overlap (assuming it is larger than some preset parameter $m$ ) are merged into one cluster. If no clusters fit this criteria, no clusters are merged.

- Step 3: Similar to the paraclique algorithm described in [47], every protein not currently belonging to a given cluster is considered for inclusion. Those proteins with connectivity proportion (the proportion of the number of proteins it is predicted to interact with in the cluster to the number of proteins it is predicted not to interact with) greater than a preset parameter $g$ are added to 
the cluster. The first time this step is run all clusters are considered but on subsequent runs only newly merged clusters are considered.

In practice, step 2 of this algorithm (the merge step) is very computationally expensive. To avoid this issue, an approximation version can be run until the number of clusters is reduced at which point the full version can be run. In the approximation version, the first two clusters with an overlap of $a$ are merged. In this case, the predicted H. sapiens interaction network was initialized with over 100,000 maximal cliques and the approximation version of this algorithm was run until the number of clusters reached 20,000. After the normal version took over, the algorithm finally produced 8,739 paracliques. For each paraclique GO term enrichment analysis was performed. With this enrichment information and the addition of a number of novel interactions tying them together as they were not before, one can assign potential functions to these proteins. Three specific examples of potentially novel protein complexes are given in Figure 5.6. Figure 5.6A shows a novel complex which one could associate with cytoskeleton and structural integrity of the cell (P-value of $\left.6.5 \times 10^{-7}\right)$. Figures 5.6B and 5.6C show novel complexes dominated by novel protein interactions which can be associated with cell adhesion (P-value of $2.2 \times 10^{-10}$ ) and the G-protein coupled signalling pathway $\left(\mathrm{P}\right.$-value of $3.75 \times 10^{-10}$ ), respectively.
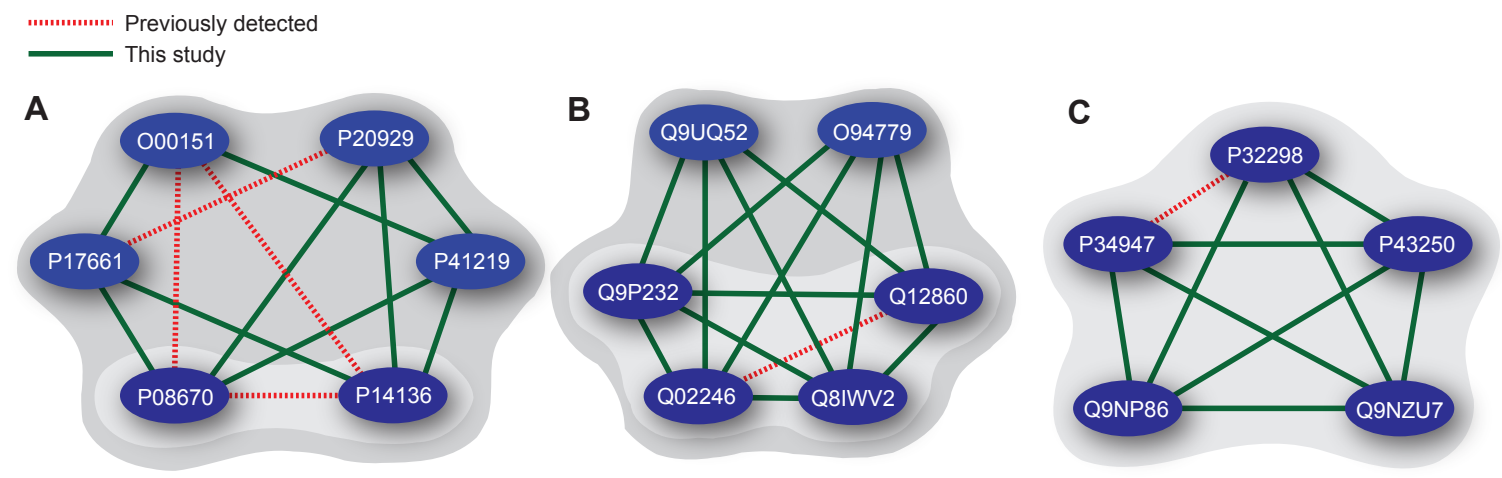

Figure 5.6: Novel protein complexes extracted from the predicted H. sapiens interaction network. Based on the associated gene ontology (GO) term enrichment, these groups of proteins could be considered as functional groups for the first time. Taken from [255]. 


\subsubsection{Demonstrating the Value of the Predicted Interactome}

After the more computationally focused, network wide analysis was performed (described in the previous sections) a closer look was made at the novel interactions in hopes of making some new biological discoveries in the data. Three different investigations will be briefly described in the following sections.

\section{Using the Predicted Human Protein Interaction Network to Identify Breast Cancer Related Proteins}

To see if the interactions predicted by PIPE could uncover new knowledge about breast cancer, proteins known to be involved in breast cancer pathways in $H$. sapiens were investigated. A mutation of the gene BRCA1 has been related to the susceptibility of brest cancer [97]. Although the tumour suppression properties of BRCA1 is well known, the molecular mechanism of its tumour suppression activities are not

fully understood [289]. A brief overview of the known members of the breast cancer pathway is given in Figure 5.7, of which BRCA1 plays a central role. As can be seen in this figure, BRCA1 is involved with the response to DNA damage (homologous recombination) and DNA damage checkpoint activity (cell cycle arrest/transition). Over 3,000 interactions were predicted involving proteins known to be involved with breast cancer, 424 of which involve BRCA1 directly (161 novel and 263 previously known interactions). Three proteins which were not previously known to be directly involved in breast cancer were Aurkb, Smc1b and Cdk3. Based on the novel predictions made by PIPE and the biological processes these proteins are known to be involved in, it was concluded that sufficient evidence existed to support the roles that these novel proteins played in breast cancer.

Four mutations on H. sapiens proteins (P04637a, P04637b, P04637c and P04637d) have been linked to the resistance of a chemotherapeutic breast cancer drug [1]. PIPE ran the mutated variants of these proteins against all of the non-mutated $H$. sapiens proteins to see if differences in their interaction profiles could provide an insight into how these mutations affect drug resistance. Two of the mutants (P04637b and P04637d) had identical interaction profiles to that of their wild types. However, the other two mutant proteins showed some differences. An example of this is the loss of interaction between the mutants and a nuclear transcription factor protein 


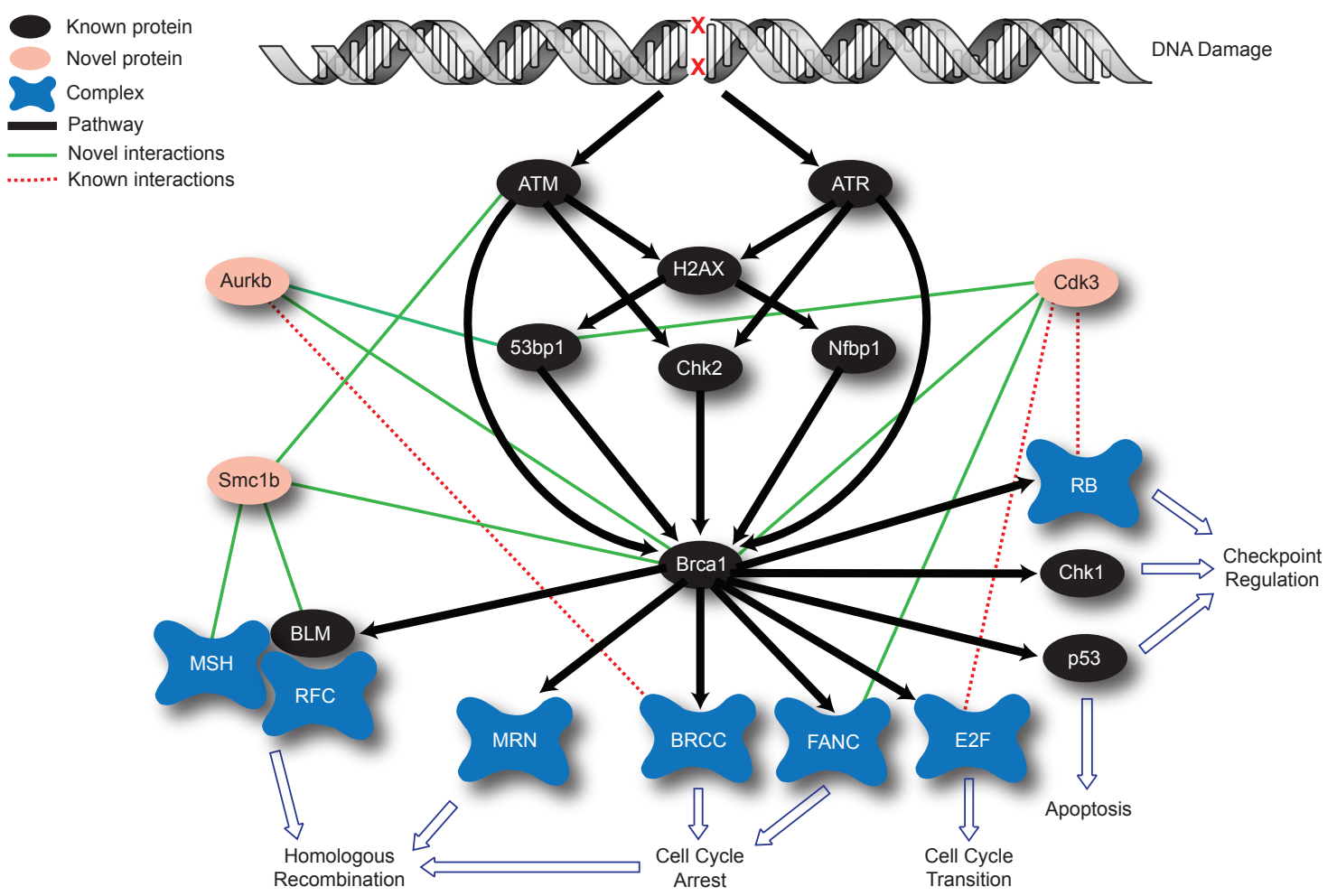

Figure 5.7: Schematic diagram of breast cancer pathway in which BRCA1 plays a central role. Black lines (edges) represent biochemical pathways, red and green edges are novel and known PPIs, respectively. Ovals and clouds represent proteins and protein complexes, respectively. Red ovals represent novel proteins that are associated with breast cancer pathway on the basis of the predicted interactions they form. Taken from [255].

(protein involved with controlling the transcription rate of DNA), a fundamental cellular process. Similarly, a truncated form of the protein P04626 is associated with breast cancer drug resistance. Compared to the wild type, this truncated version of the protein was predicted to lose interactions with some proteins involved in key cellular processes. For more details and biological analysis, please see [255].

\section{Identification of Novel Molecular Markers for Seasonal Allergic Rhinitis}

Glucocorticoids are a steroid hormone commonly used in the treatment of patients with Seasonal Allergic Rhinitis (SAR) [311]. Protein markers can be used to monitor one's response to this treatment, however identifying these markers is difficult 
due to the sheer number of proteins involved in SAR. Because of this, proteins related to SAR were investigated within the predicted $H$. sapiens interaction network. From the literature, 191 proteins involved in biological processes related to SAR were collected and proteins that were predicted to interact with ones from this set were identified. 3,334 proteins were predicted to interact with at least one of these SARrelated proteins. Five proteins (PRB1, PRB2, SFN, LYN and Akt2) with a high number of predicted interactions to the SAR-related proteins were targeted as potential biomarkers. Using ELISA, these candidates were analyzed in nasal fluid from 40 patients with SAR before and after treatment with Glucocorticoids. Akt2 and SFN were not detectable in most samples and PRB2 was not deferentially expressed before and after treatment. On the other hand, the concentration of LYN increased and PRB1 decreased significantly after treatment. This suggests that LYN and PRB1 could be used as novel molecular markers for SAR and the potential exists for using other novel PIPE predictions to identify biomarkers for other human conditions.

\section{Identification of Novel Proteins Involved in Translation}

The process of protein synthesis, or translation, is a fundamental cellular mechanism and is therefore well conserved over a variety of different organisms. When inspecting the predicted $H$. sapiens interaction network, five proteins with novel predicted interactions with proteins known to be involved in translation were identified (Q96DG6 (CMBL), Q08AM6 (VAC14), P23511 (NFYA), Q9UKR5 (ERG28) and P48735 (IDH2)). Since this process is so well conserved, the homologs of these proteins (AIM2, VAC14, HAP2, ERG28 and LYS12, respectively) in S. cerevisiae were investigated. This was done because $S$. cerevisiae was much more amenable to wet-lab experiments at the time. First, the effect of the deletion of these genes was shown to significantly alter the cell's ability to detect all stop codons during translation. Further deletion studies were done on HAP2, ERG28 and LYS12 and it was shown that these mutants displayed altered levels of sensitivity to drugs that affect translation. For further details and experimental setup, please see [255]. 


\section{Chapter 6}

\section{Using Predicted PPI Networks to Gain Insights into the Evolution of Closely Related Organisms}

Evolution is the process by which organisms have developed from simpler forms of life into more complex entities. The random mutations which occur to the genetic information of an organism, followed up by natural selection (the process whereby individuals of a population who have gained some advantage over others through random mutations become more fit to survive in their environment) is the relatively simple process from which the diversity of life found on earth was born. Although this process is well understood at a high level, the quest to understand how mutation affects organisms at a cellular level, specifically which of these mutations are responsible for phenotypic changes in an organism, has been pursued by members of the scientific field for decades.

The field of comparative genomics aims to compare and contrast organisms by investigating the genomes of closely related species. When presented with two or more relatively similar organisms, the traditional approach to investigate them would be to directly compare their genome sequences though whole genome alignments. From this one could infer many different things, including which genes are well conserved between the organisms, which have undergone some degree of mutation, those genes that are only present in a single organism due to the evolutionary distance between the organisms as well as the overall effect these mutations have on the proteins some of these genes code for $[150,241,249]$. These studies have provided not only a degree of insight into the evolution of certain organisms, they have also provided a significant amount of raw data useful to the scientific community as a whole with the introduction of next-generation sequencing methods in the past decade.

Although these approaches have yielded tremendous insight into the evolution of independent genes, it is more recently being acknowledged that the evolution of a given gene or protein is heavily impacted by its interactions with other genes or 
proteins and its role in its respective interaction network [118, 141, 208, 272]. Because of this, interest into the study of network evolution (through empirical [130, 142, 238], computational [284] and theoretical [65] approaches) has increased in recent years. However, the cost, time, expertise and relatively high error rates involved in high throughput experimental methods of PPI network determination have lead to a lack of high quality, comparative PPI data.

The aim over the following sections is to show that it is possible to supplement the knowledge gained though comparative genomic methods with the analysis of predicted PPI networks. First, the molecular basis for the emergence of multicellularity within the volvocine algal lineage (containing the unicellular Chlamydomonas reinhardtii, the colony forming Gonium pectorale with undifferentiated cells and multicellular Volvox carteri) will be investigated. Secondly, a set of five closely related strains of yeast $(S$. bayanus, S. kudriavzevii, S. mikatae, S. paradoxus and S. cerevisiae) will be studied and the notion of a null model for this type of analysis will be introduced. In both cases, the predicted PPI interaction networks will be analyzed in hopes of adding evolutionary information not readily available through the standard comparative genomic analysis.

\subsection{Identifying Potential Protein Clusters within a PPI Network}

A protein cluster is a group of proteins that come together, through interaction, to perform a specific function or partake in some biological process and are thus of great interest. In the context of a PPI network, a potential protein cluster would look like a dense subgraph, where a great deal of the proteins interact with one another. In other contexts, such as social networking for example, these clusters have been referred to as communities, paracliques and pseudocliques, amongst other things. In any case, the search for dense subgraphs is a well studied problem and many approaches have been made to identify them, all with their own definition of what constitutes a cluster. Traditionally these methods attempted to partition the graph into disjoint clusters $[54,82,153]$ however, more recently, there has been more interest in detecting overlapping clusters in networks [292]. In the context of PPI networks, searching for overlapping clusters instead of focusing strictly on disjoint clusters makes sense as there is no reason that proteins can not be involved in multiple clusters. In this 
section an overview of available algorithms aiming to identify overlapping clusters in a graph, as well as a description of the algorithm chosen to identify protein clusters in the work presented here, will be given. Focus will be given to methods with readily available implementations as well as those producing well-defined sets of clusters, as opposed to "fuzzy" clustering approaches that produce soft membership estimates for nodes with respect to the set of clusters.

Methods based on clique percolation work on the assumption that clusters are made up of the union of overlapping, adjacent cliques. Palla et. al developed an algorithm that identifies $k$-clique-communities, which is defined as the union of all $\mathrm{k}$-cliques (cliques of size $k$ ) which can be reached through all adjacent $k$-cliques. Two $k$-cliques are said to be adjacent if they share $k-1$ members [205]. Through experimental runs, it was found that the running time of their algorithm is very dependant on the structure of the input graph and no estimation of the runtime can be given without the input readily available. CFinder [3] is the publicly available implementation of their algorithm. Farkas et. al extended the idea of clique percolation to weighted graphs, in which $k$-cliques are only included into a community if their "intensity" (based on edge weights) is above some set threshold [77].

Another way to look at the problem is to focus on the edges of the graph and cluster them. A node would then fall into two clusters if it is adjacent to edges in two different clusters. Evans and Lambiotte presented a method which projects the given graph one wants to cluster onto a line graph (a line graph of a graph $G$ is a secondary graph which represents the edges as nodes where two nodes are connected if the edges they represent have a node in common). In doing this, one can then run any disjointed cluster identification algorithm on the line graph which would then produce an overlapping set of clusters in the original graph [74]. Evans then extended the idea of a line graph to a clique graph (where cliques are represented as nodes in a weighted graph and the edge weights are based on the amount of clique overlap). As before, a vertex partitioning in the clique graph results in an overlapping clustering of the original graph [73]. Ahn et. al introduced a method that compares edges based on the neighbours of the nodes the edges are adjacent to. Similar nodes are placed into clusters in a hierarchical manner. At the lowest level one has just a set of clusters, but as one travels up the hierarchy, larger groups of related edges are revealed which 
are essentially clusters of clusters [4].

COPRA [92] is method which aims to propagate information through the network to determine clusters. At the start, each node has a unique ID in its memory. Each node maintains a list of IDs as well as a "belonging coefficient", indicating the degree to which the node is a part of the cluster with that ID. The algorithm runs for a series of generations, during which each node updates its "belonging coefficients" by averaging the coefficients of its neighbours. After a predermined number of iterations, the algorithm stops and each node locally decides which cluster it is a part of. The Speaker-Listener Label Propagation Algorithm (SLPA) [293, 294, 296] is similar to COPRA. At the start each node's memory is set to its own unique ID. Nodes are then chosen at random to be listeners. All of these nodes' neighbours choose a label to send from their memory and the listener accepts one of these labels to add to its memory. After each node has been a listener a certain number of times, the algorithm stops. The nodes then decide which clusters they are in based on what is in their memory. The method was then updated to LabelRank [295] which removes all randomness involved with SLPA (ie. when a node decides what to send or what to store) such that the clustering produced for a given graph is always the same.

Chen et. al developed a game-theoretic framework to identify clusters in a graph. In this framework, each node acts as a selfish agent who selects which clusters to join or leave based on its own internal calculations. The algorithm runs in a series of iterations, where during each iteration each node decides to leave, join or switch to another cluster until a state of equilibrium is reached across the graph [43].

Methods based on local expansion and optimization look to identify clusters by growing communities from seeds (sometimes randomly chosen), through successively adding neighbouring nodes and aiming to optimize some scoring function. Lancichinetti et. al introduced a method that grows clusters from randomly chosen seeds which aim to maximize a fitness function based on the number of internal and external edges a cluster has. Once the fitness function for a given cluster cannot be increased by adding any neighbouring nodes, the process is repeated from another randomly chosen location in the graph until no more clusters can be formed. These clusters are then clustered again, forming a hierarchy of clusters showing which clusters are highly connected to one another [147]. iLCD is a similar approach where the 
seeds of clusters are identified as groups of three or four nodes conforming to specific interaction patterns. From these seeds, the algorithm then adds neighbouring nodes or tries to merge with neighbouring clusters based on their combined density. iLCD is also able to adapt to changing networks, where nodes and edges are added and the clusters are then updated at regular intervals [32]. Nepusz et. al introduced a similar method which expands a complex from a single seed vertex by adding or removing vertices in a greedy manner to find a highly connect subgraph. This process is repeated from a number of different seeds to produce a set of overlapping clusters. These clusters are then compared in a pair-wise manner and clusters which have a considerable overlap are merged. Lastly, clusters that are below a set size or density threshold are discarded [194]. Jin et. al presented UEOC (unfold and extract overlapping communities) which builds a cluster from a given seed through a Markov random walk method combined with a constraint strategy based on an annealed network (having no community structure) corresponding to the original input graph. This ensures that the random walks taken are constrained to dense subgraphs. The end of a given walk will correspond to a newly discovered cluster [127]. EAGLE is a similar approach to these local expansion algorithms which also produces a hierarchy of clusters. Its major difference is that the initial seeds used to identify clusters are maximal cliques. The first stage of the EAGLE algorithm builds the hierarchy of clusters, or dendrogram, which is then split up into communities. This stage first finds all maximal cliques in the graphs and calculates the similarity (based on connecting edges) between all pairs of cliques. Next, the most similar clusters are merged into one cluster and the cluster's new similarity is calculated with respect to all of the other clusters. This process continues until one cluster is left, forming a natural hierarchy. In the second step, this hierarchy is cut to produce a set of clusters. This cut is done at a location which gives the best coverage over the initial graph [258]. Greedy Clique Expansion (GCE) is a method very similar to EAGLE. GCE is seeded with a set of maximal cliques and then these cliques are expanded in a greedy fashion. Each cluster considers all neighbouring nodes and chooses the neighbour which is connected to the nodes making up the cluster the most. If adding this node to the cluster improves the cluster's fitness, it is added. If not, the algorithm moves onto the next cluster until all clusters are formed. GCE finishes by removing clusters that 
are determined to be too similar to previously discovered clusters [151].

The Order Statistics Local Optimization Method (OSLOM) [148] is another local expansion and optimization method for cluster identification with a significant novel addition. The goal of OSLOM is to find statistically significant clusters in the graph and it does this through the use of a null model. In this case, the null model is built from random graphs with a given node degree distribution, where each node has the same number of neighbours as it had in the original graph (although randomly assigned). When given a cluster and a node $i$ that one is considering to add to the cluster, the null model can be consulted. If the node $i$ shares many more edges with the cluster in the original graph than in the null model it can be considered for inclusion into the cluster as the connections between $i$ and the cluster are unexpectedly strong. This idea was incorporated into the fitness function used to evaluate clusters. OSLOM starts at a random node in the graph and grows a cluster around this point. To grow a cluster, it calculates the cumulative probability of each of its neighbours having the number of connections to the members in the cluster equal or larger than observed in the null model. The neighbouring nodes which are considered significantly linked to the cluster are added. During this process, however, current members of the cluster may no longer be significantly connected to the new version of the cluster as it now stands. To rectify this, the significance of every member is reevaluated and non-significant nodes are dropped. This process is continued until no members are added to the cluster. OSLOM then attempts to build another cluster starting at a new, random point until the graph has been completely explored. As a final cleanup step, OSLOM tries to merge similar clusters in an attempt to avoid having nearly identical clusters in its output. Like other methods mentioned here, OSLOM then continues clustering the clusters found in the first iteration to build a hierarchy of clusters. Due to the fact that OSLOM inherently guarantees that its output consists of statistically significant clusters that are unlikely to be found in a random graph with the same degree distribution (unlike the other methods), it was chosen as the tool to find potential protein clusters within PIPE's predicted PPI networks. Although the quality of a clustering algorithm's output for a given graph is hard to compare to another method (due to the fact that they all use a different definition of what a cluster is and there is no one definition that is considered perfect), OSLOM was independently 
compared with a variety of other community detection methods and was found to perform comparably, if not better, than other methods tested. In particular, OSLOM was among the top performers when tested on real-world graphs [292]. Due to the fact that the OSLOM program is readily available and is relatively efficient, contains the novel idea of a built in null model that no other method employs, and has been independently tested and shown to be one of the top performing clustering methods on real-world graphs, it was chosen as the tool used to cluster the predicted PPI networks in the projects described in the next few sections.

\subsection{Studying the Evolutionary Transition in Individuality of Multicellularity in the Volvocine Lineage of Green Algae using Predicted PPI Networks}

The diversity and complexity of life on earth is a direct result of the evolutionary process, allowing simpler organisms to transition into more complex ones. Evolutionary transitions in individuality (ETIs) are major milestones in the evolutionary process and represent significant transitions in organismal complexity. The transition of an organism from unicellularity to multicellularity is one of the most interesting and important ETIs. This transition allows for organisms at one level of complexity (unicellular) to evolve into more complex organisms (multicellular), largely contributing to the variety of life seen on a day to day basis [181]. Although this phenomenon is of great interest to the scientific community, it is one that is very difficult to study as most transitions of this type occurred in the distant past. As the amount of time since an organism made this transition grows, the harder it is to distinguish the genetic changes that caused the transition from the changes that occurred post-transition [182]. Luckily, the volvocine family of green algae is well suited to the study of this ETI, as a relatively complete timeline of this transition is known [108]. This family contains the unicellular Chlamydomonas reinhardtii [179] and the multicellular Volvox carteri [227] organisms, both of which exist in nature today [138]. It is believed that the most recent ancestor of these organisms was also unicellular and was relatively similar, at the genetic level, to Chlamydomonas [109, 139].

Recently, work has been done to sequence both Chlamydomonas and Volvox and analyze the resulting genetic codes though the conventional comparative genomics 
method of whole genome alignments [227]. Despite the vast differences in biological complexity, when the Volvox and Chlamydomonas genomes were compared, very little difference was found. The size of their genomes, the number of protein coding genes, the types of domains encoded in their proteins as well as their respective gene families were all found to be very similar [182]. Work has been done on a small number of specific genes and gene families in the past decade, comparing them between the two organisms and finding notable differences [64, 183, 197, 277, 278], however how these specific genetic differences translate into phenotypic changes on the basis of PPIs and interactome complexities has not been described. Even more recently Gonium pectorale, a unicellular organism in the volvocine family that forms colonies of undifferentiated cells, was sequenced and analyzed. Using these three organisms the transition from unicellularity to multicellularity can be studied. The multicellular Volvox and the unicellular Chlamydomonas (which is believed to be very similar to the unicellular ancestor of this group of algea) represent logical endpoints with Gonium representing a key intermediary stage of this transition [103].

In an attempt to supplement the evolutionary insights gained through individual gene and gene family studies between Chlamydomonas and Volvox, the predicted interaction networks for these two organisms, along with Gonium, will be investigated with the aim of finding significant differences at a molecular systems level. Two key concepts in developmental biology will be focused on here, namely modularity [51] and integration [222]. Modularity refers to the degree to which a network (here a PPI network) is split up into functional units and how tightly connected these units are internally whereas integration refers to how these distinct units interact with one another, both of which are known to impact evolvability [13, 140]. It has been identified that modularity is a key aspect of the evolvability of an organism [221] because it allows sub-units of the overall network to change independently of one another (instead of single mutations having a network-wide effect), allowing nature to specifically tune the internal workings of each sub-unit [104] while also making it easier to rewire these functional units with one another [51]. The way in which genetic mutations of a given organism map to changes in phenotype (here PPIs or protein clusters) is of central concern to those studying evolution [221], with modularity and integration becoming key concepts for studying organismal phenotypes, specifically how modular 
networks evolve and how modularity affects this evolution [172]. Two other unicellular chlorophytes, Chlorella viriabilis and Coccomyxa subellipsoidea, which branched off the volvocine lineage prior to the other volvocines studied here, are included for comparative purposes. The work described here has been submitted and is being considered for publication in the journal Scientific Reports [254]. Biological motivation and conclusions for this project courtesy of collaborators Dr. Pierre Durand and Jonathan Featherston from the faculty of Health Sciences at the University of Witwatersrand, Johannesburg.

\subsubsection{Computational Setup, Initial Data Sets \& Predicted PPI Networks}

As is typical for these studies, there are very few known Chlamydomonas, Volvox or Gonium PPIs and a related model organism with extensive empirical data can be used to supply the known interactions for a reference PIPE database, as was seen in Section 5.1 and in [225]. Here, PPIs for the flowering plant Arabidopsis thaliana were used. Arabidopsis was chosen as it is the most highly studied photosynthetic organism and thus makes it a good candidate to study other photosynthetic organisms like algae. Empirically proven, high quality interactions were obtained from The Arabidopsis Information Resource (TAIR) [146]. The N-Browse list of PPIs was used as this list consists only experimentally verified interactions. Isoforms were then removed leaving 4,781 known interactions which formed the basis for the MP-PIPE database in this project.

Protein sequences and annotation information for Volvox, Chlamydomonas, Chlorella and Coccomyxa were obtained from the Joint Genome Institute [198] and Phytozome (version 9.1) [90]. Version 1 and version 5.3 genomes and annotations were used for Volvox and Chlamydomonas, respectively. The Gonium protein se-

quences used in this study are the result of recent whole genome sequencing [103]. The protein sequences of each organism were clustered using CD-HIT [85, 157] at 90\% similarity leaving 14,779 Volvox, 16,800 Chlamydomonas, 17,404 Gonium, 9,638 Chlorella and 9,385 Coccomyxa sequences. A reciprocal best hit BLAST mapping for the individual proteins between each pair of main organisms was created. These mappings successfully mapped the following numbers of proteins uniquely between 
the three pairs of organisms: Volvox - Chlamydomonas: 8,958; Volvox - Gonium: 8,757; and Chlamydomonas - Gonium: 9,979. The intersection of these mappings is the set of proteins that map perfectly across all three organisms. The number of proteins that fit this description was 7,106 .

Since there are very few known interactions in any of the three organisms, the traditional LOOCV tests to set an appropriate cutoff with a specific sensitivity/specificity could not be done. An estimated MP-PIPE cutoff parameter setting, derived from experiments on H. sapiens, S. cerevisae and C. elegans where experimental interaction data is available, was therefore used. LOOCV tests, as described in Section 3.2.3, were performed. An operating point at a specificity of $99.95 \%$ was chosen and the associated cutoff parameter value used to build the interaction graphs for H. sapiens, S. cerevisae and C. elegans. At a specificity of 99.95\%, MP-PIPE achieved a sensitivity of $27.83 \%, 16.40 \%$ and $13.49 \%$ respectively. For each interaction graph, the density refers to the number of interactions present as a percentage of the total number of possible interactions. At the chosen operating point, the average density of the interaction graphs for $H$. sapiens, S. cerevisae and C. elegans is $0.1764 \%$. To determine the interaction graphs for the organisms studied here, the respective PPI predictions were sorted by their PIPE interaction scores and the top $0.1763 \%$ were taken as the predicted PPI network. Note that, by this chosen method, the predicted interaction networks for the organisms studied here all have the same edge density. This is important for further analysis because it ensures that differences in edge density within clusters or between clusters are not artifacts of differences in overall edge density.

\subsubsection{Specificity Validation}

Six proteins with no expected interactions between them (based on their annotations) that exists in Volvox were chosen to act as a spot check for the specificity of the predicted interaction networks. Five of these proteins have homologs in all three organisms, as shown in Table 6.1. This meant that there were 15 Chlamydomonas, 15 Gonium and 21 Volvox expected negative pairs in total, including self-interactions. Two of the 51 expected negative pairs were predicted to interact using PIPE (Gonium proteins scaffold00002.g1124 \& scaffold00015.g414 and a self interaction with 
Chlamydomonas protein g673650), giving a specificity of $96 \%$ for this test. The possibility also exists that the two predicted interactions are accurate but no empirical evidence has been obtained to prove it.

\begin{tabular}{lccc}
\hline Homologous protein & Chlamydomonas & Gonium & Volvox \\
\hline $\begin{array}{l}\text { phosphoenolpyruvate } \\
\text { carboxylase 3 }\end{array}$ & & 59445 \\
$\begin{array}{l}\text { light harvesting complex } \\
\text { of photosystem II 5 }\end{array}$ & g673650 & scaffold00002.g1124 & 104309 \\
$\begin{array}{l}\text { threonine aldolase 2 } \\
\text { ribosomal protein S10 }\end{array}$ & g423550 & scaffold00006.g797 & 94060 \\
$\begin{array}{l}\text { vacuolar protein sorting- } \\
\text { associated protein 2.3 }\end{array}$ & g735400 & scaffold00015.g414 & 108519 \\
$\begin{array}{l}\text { sphingoid base } \\
\text { hydroxylase 2 }\end{array}$ & g103500 & scaffold00019.g369 & 57952 \\
\hline
\end{tabular}

Table 6.1: A set of proteins expected not to interact with one another in Chlamydomonas, Gonium and Volvox. Of the 51 expected negative pairs, PIPE incorrectly predicted two of them to interact (Gonium proteins scaffold00002.g1124 \& scaffold00015.g414 and a self interaction with Chlamydomonas protein g673650).

\subsubsection{Results and Discussion}

First, the predicted PPI networks were compared to identify phenotypic differences. The number of interactions for each protein in the 7,106 homologs identified across the three volvocine organisms via reciprocal best hit BLAST mappings were counted in their respective interactomes. Figure 6.1 shows a heatmap comparison between each pair of volvocine organisms. Figure 6.1A compares Chlamydomonas to Gonium, Figure 6.1B compares Chlamydomonas to Volvox and Figure 6.1C compares Gonium to Volvox. 

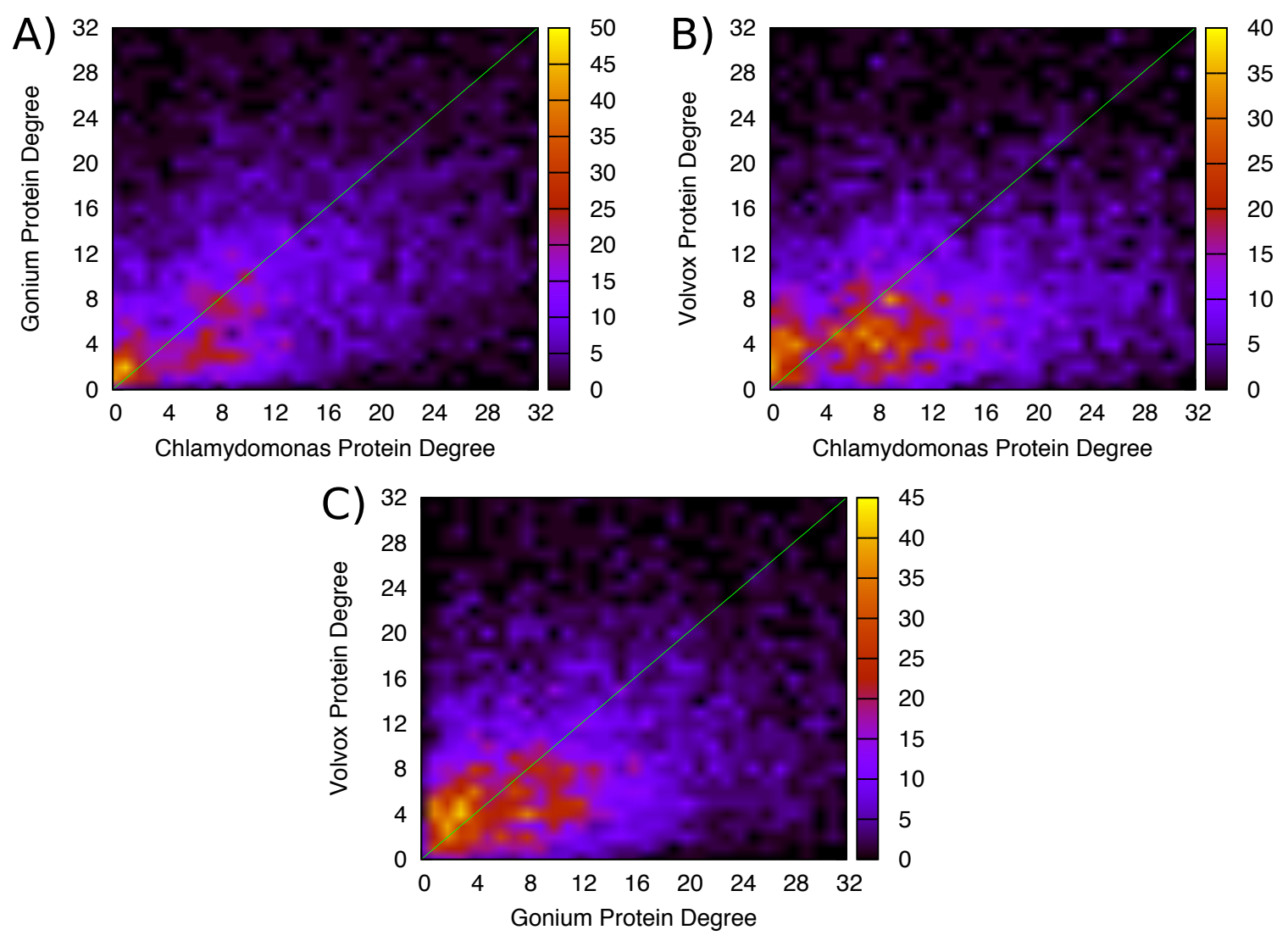

Figure 6.1: Pairwise comparison of degrees of homologous proteins between Chlamydomonas, Gonium and Volvox. A compares Chlamydomonas to Gonium, B compares Chlamydomonas to Volvox and C compares Gonium to Volvox. In each plot, the colour intensity of a given location indicates the number of proteins with the indicated degree in the respective intertactomes. Homologs lying on the green line in each plot have the same degree in both networks.

Overall Chlamydomonas has more proteins with 8-32 interactions than Gonium while Gonium has more proteins with less interactions (1-8) than Chlamydomonas. Volvox appears to have more proteins with 5-12 interactions than Chlamydomonas, whereas the latter has more proteins with very few interactions (1-4) than Volvox. There seems to be very few significant differences between Gonium and Volvox. To further investigate and quantify the differences between the interactomes, the individual PPI networks were then clustered using the OSLOM method [148], which found 190 Chlamydomonas, 291 Gonium, 167 Volvox, 173 Chlorella and 195 Coccomyxa clusters with a minimum size of 4 proteins per cluster. Figure 6.2 visualizes the degree to which portions of the PPI networks can change between the organisms studied 
here. The green nodes are proteins making up a randomly chosen Chlamydomonas cluster. The solid lines represent predicted PPIs with the dashed lines representing those proteins with significant sequence similarity mapped to one another via the reciprocal best hit BLAST mappings. When comparing Chlamydomonas and Gonium in this figure, the densely connected cluster lost a few proteins and a significant number of predicted PPIs. A further protein was lost on the transition from Gonium to Volvox as well as further changes in predicted PPIs. These kinds of changes can be expected throughout the PPI networks.

As previously stated, measures of modularity and integration were key focal points of analysis for the predicted PPI networks. Modularity was quantified by the number of protein clusters found $\left(N^{m}\right)$ and their average internal interaction rate $(I)$. Integration was measured by the average external interaction rate of protein clusters (percent of proteins in the rest of the proteome a given cluster is connected to) (E) as well as the average cluster integration rate (the average percentage of clusters a given cluster is connected to, either by protein sharing or direct PPIs between member proteins) $\left(M^{i}\right)$. The average number of proteins per protein cluster was also measured $\left(N^{p}\right)$. Both $N^{m}$ and $N^{p}$ were normalized by the number of proteins in their respective PPI networks to allow for direct comparison. The others do not require normalization as they are given as percents. A summary of these measures and other statistics regarding each organism is given in Table 6.2 and Figure 6.3. Figure 6.4 displays the network measures of modularity and integration across the protein clusters in their respective organisms as cumulative histograms.

Along the trajectory of unicellularity to multicellularity, the modularity measures (the number and density of clusters, $N^{m}$ and $I$ respectively) increased while the measures of integration (how connected clusters are to one another and to the rest of the PPI network, namely $M^{i}$ and $E$ ) decreased. This suggests that increasing modularity and organism complexity, paired with decreasing integration between protein clusters and the rest of the PPI network may capture a systems level understanding of the ETI in these organisms. The increase in organismal complexity correlates positively with increasing modularity at the expense of a decreased integration between protein clusters and other clusters/proteins (negative correlation). This increasing number of less connected modules in the cell makes sense when thinking about the organisms 


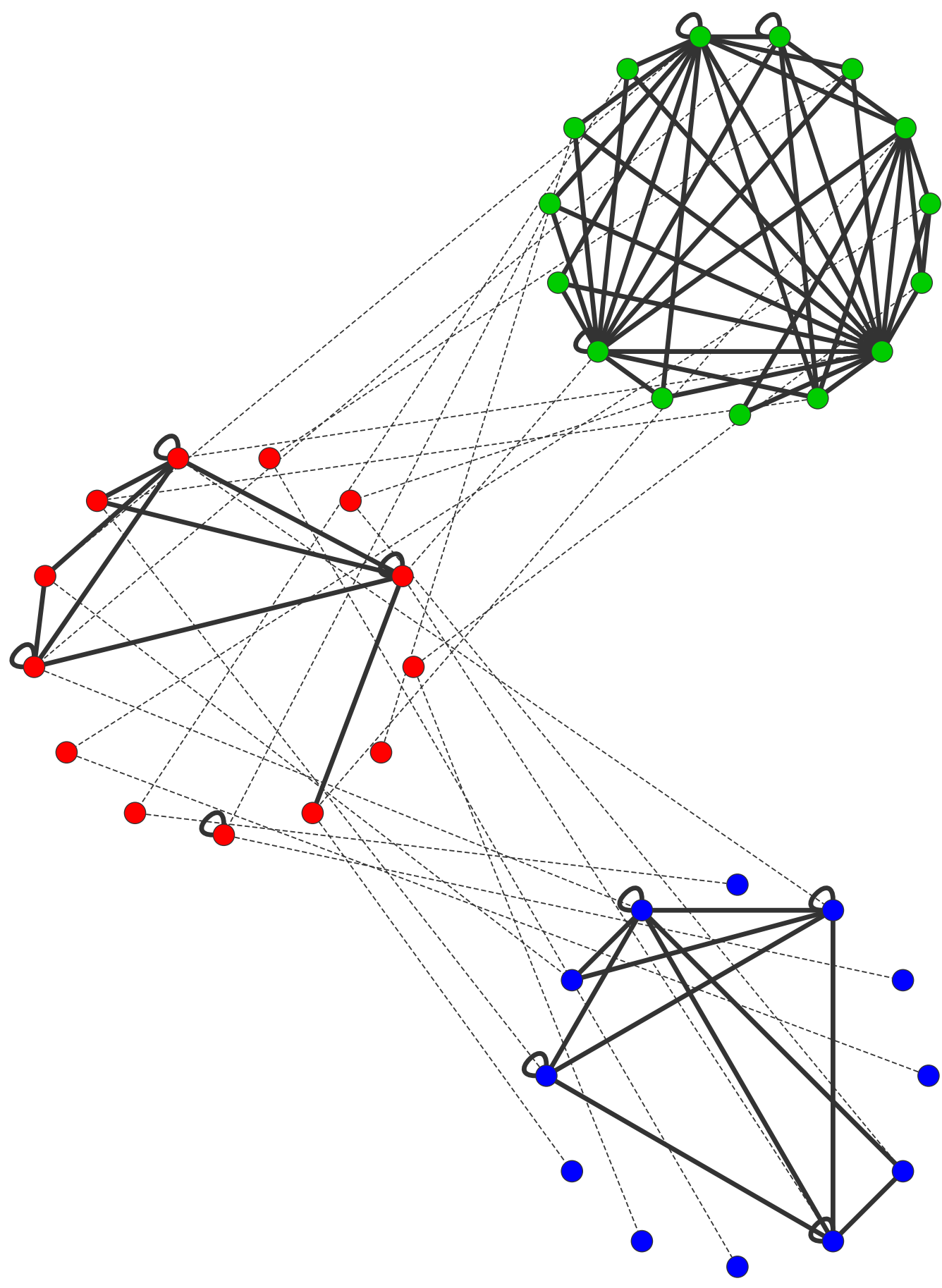

Figure 6.2: Visualization of the changes between PPI networks. Green, red and blue nodes represent Chlamydomonas, Gonium and Volvox proteins, respectively. Solid edges represent predicted PPIs within a given organism and dashed edges represent reciprocal best hit BLAST mapped proteins between organisms. 


\begin{tabular}{|c|c|c|c|c|c|}
\hline & Chlamy. & Gonium & Volvox & Chlorella & Coccomyxa \\
\hline $\begin{array}{l}\text { \# of proteins } \\
\text { (after clustering) }\end{array}$ & 16,800 & 17,404 & 14,779 & 9,638 & 9,385 \\
\hline$\#$ of interactions & 248,916 & 267,136 & 192,632 & 81,927 & 77,683 \\
\hline $\begin{array}{l}\text { Normalized \# } \\
\text { of interactions }\end{array}$ & 14.8164 & 15.3491 & 13.0342 & 8.50042 & 8.27736 \\
\hline \# of clusters & 197 & 292 & 257 & 173 & 195 \\
\hline $\begin{array}{l}\text { Normalized \# } \\
\text { of clusters }\left(N^{m}\right)\end{array}$ & 0.01173 & 0.01678 & 0.01739 & 0.01795 & 0.02078 \\
\hline $\begin{array}{l}\text { Avg \# proteins } \\
\text { per cluster }\end{array}$ & 81.8680 & 60.7360 & 58.9416 & 50.9595 & 45.2769 \\
\hline $\begin{array}{l}\text { Normalized average } \\
\text { \# proteins per } \\
\text { cluster }\left(N^{p}\right)\end{array}$ & 0.00487 & 0.00349 & 0.00399 & 0.00529 & 0.00482 \\
\hline $\begin{array}{l}\text { Average } \% \text { of internal } \\
\text { cluster interactions } \\
\text { present }(I)\end{array}$ & 20.5929 & 24.5382 & 25.8927 & 24.5334 & 24.5777 \\
\hline $\begin{array}{l}\text { Average } \% \text { of external } \\
\text { cluster interactions } \\
\text { present }(E)\end{array}$ & 0.23449 & 0.21095 & 0.20561 & 0.20397 & 0.20546 \\
\hline $\begin{array}{l}\text { Average cluster inte- } \\
\text { gration }(\% \text { of connected } \\
\text { clusters) }\left(C^{i}\right)\end{array}$ & 87.3818 & 77.2917 & 70.9322 & 71.1551 & 65.9619 \\
\hline
\end{tabular}

Table 6.2: Summary of predicted PPI network statistics for Chlamydomonas, Gonium and Volvox as well as Chlorella and Coccomyxa (included for comparative purposes). Bolded entries represent key network parameters with respect to modularity and integration. 


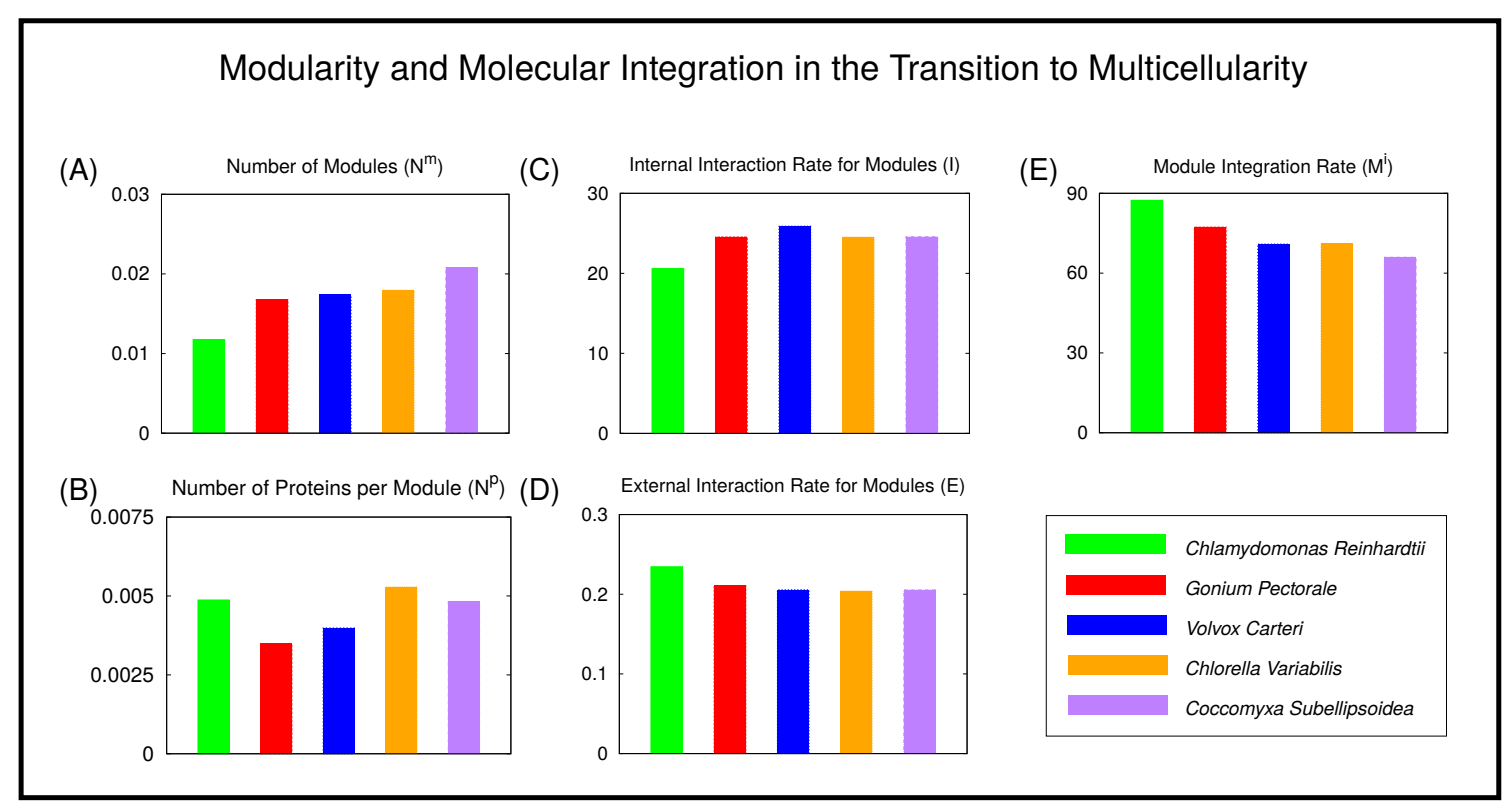

Figure 6.3: Visual representation of the key network parameters with respect to modularity and integration (namely: A - normalized number of modules $\left(N^{m}\right)$, B normalized number of proteins per module $\left(N^{p}\right), \mathrm{C}$ - internal interaction rate (\%) for modules $(I)$, D - external interaction rate $(\%)$ for modules $(E)$, E-module integration rate $\left.(\%)\left(M^{i}\right)\right)$ for the predicted PPI networks for Chlamydomonas, Gonium and Volvox as well as Chlorella and Coccomyxa (included for comparative purposes).

as a whole. In a unicellular organism, the cell is the individual and therefore must be a fully integrated unit as it does not participate as a part of a larger system. On the other hand however, cells of a multicellular individual are a part of a larger overall system and, since each cell performs different functions in this system, the individual cannot be as highly interconnected. The average size of a protein cluster $\left(N^{p}\right)$ shows no correlation with complexity.

There are two major caveats with this analysis. Firstly, only three of the volvocine organisms are studied here. To be able to make a more general statement on this ETI other members of this family need to be studied. Secondly, one can not definitively state that these results can be attributed to the ETI itself as opposed these findings being a result of adaptation done by the existing species post transition. Essentially, it is not known for certain how closely the proxy organisms used in this study resemble their extinct counterparts at the protein/PPI network level. That said, it is safe to assume that the extinct organisms that gave rise to the multicellular organisms like 


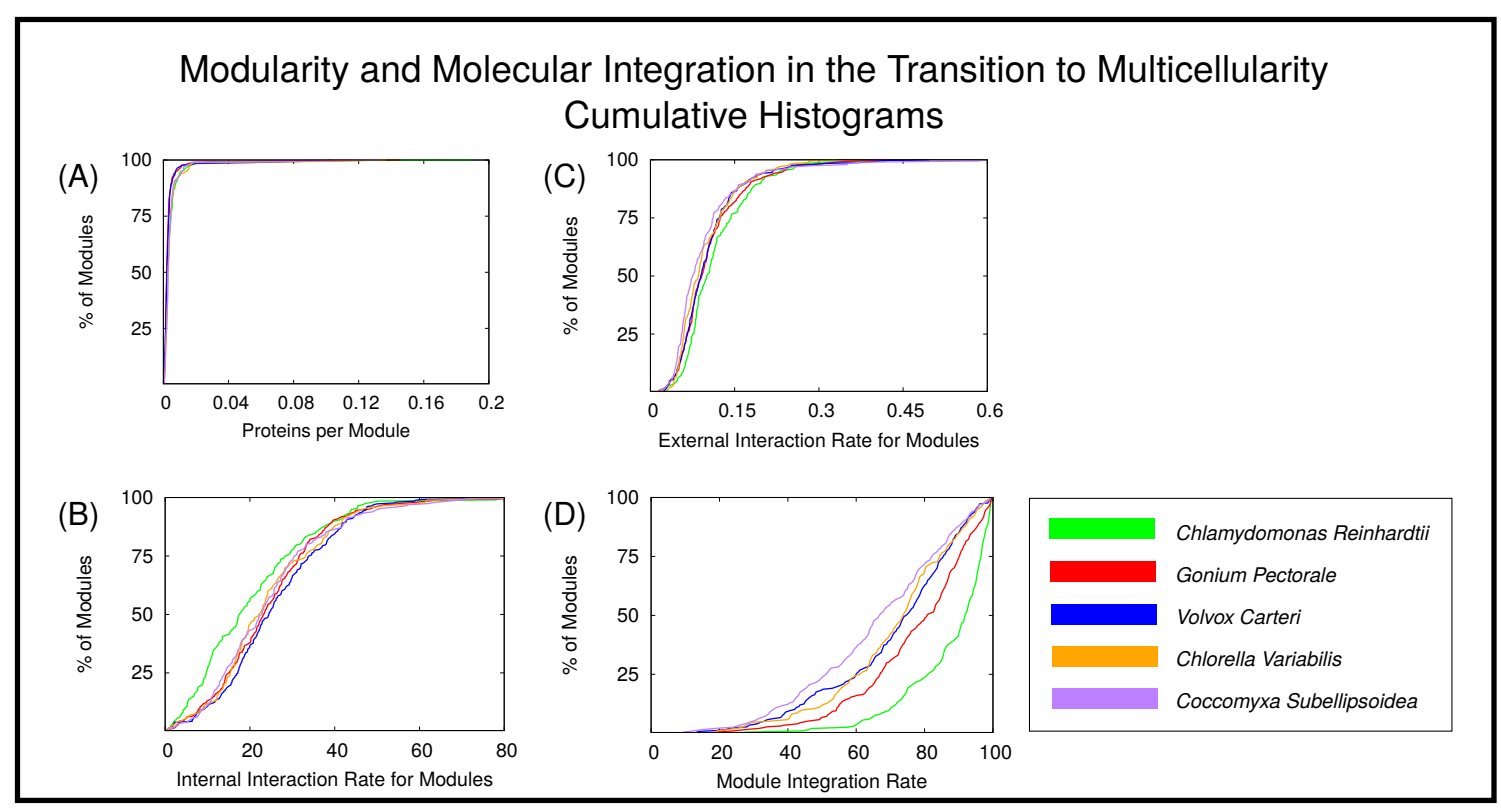

Figure 6.4: Cumulative histograms of the key network parameters with respect to modularity and integration (namely: A - normalized number of proteins per module $\left(N^{p}\right)$, B - internal interaction rate $(\%)$ for modules $(I), \mathrm{C}$ - external interaction rate $(\%)$ for modules $(E)$, D - module integration rate $\left.(\%)\left(M^{i}\right)\right)$ for the predicted PPI networks for Chlamydomonas, Gonium and Volvox as well as Chlorella and Coccomyxa (included for comparative purposes).

Volvox must have passed through similar steps of complexity that are mirrored by the volvocine organisms seen in nature today. For this reason, the findings here in the extant species should reflect the ancestor organisms to some degree.

The two other unicellular chlorophytes had more protein clusters than Volvox with cluster density in the range of Gonium and Volvox. In terms of integration, their measures were again similar to Volvox. Taken together, this data indicates that these unicellular chlorophytes exhibit even greater modularity and less integration and are thus very unlike Chlamydomonas. The reason for this is unclear however it is possible that high modularity and low integration may have played a part in the evolution of the volvocines studied here but not these unicellular chlorophytes. As can be seen in Figure 6.4, across the range of protein clusters in each organism the various parameters studied were relatively consistent across the organisms except for module integration rate $\left(M^{i}\right.$, Figure 6.4D). Here a significant differences between the organisms, with the greatest difference being between the unicellular chlorophytes and Chlamydomonas, can be seen. The impact that this may have had on multicellularity 
is, again, unknown but it does hint at the notion that integration plays an important role in this ETI.

\subsection{The Study of Closely Related Yeast Strains \& the Introduction of a Novel Null Model for Comparing Predicted PPI Networks}

In 2011, Scannell et al published improved, high quality genome sequences for the yeast strains S. bayanus, S. kudriavzevii, and S. mikatae and also provided highquality alignments of the genomes with better studied strains S. paradoxus and $S$. cerevisiae. In doing this, they identified a set of 4,179 orthologous protein-coding genes in all 5 organisms [249]. Although an undoubtedly good staring point, the analysis provided was done only on the gene/protein level. The goal here is to supplement this work by investigating the predicted PPI networks for these yeast stains with the aim of finding additional information not readily available though classical comparative genomics analysis. Since, relative to $S$. cerevisiae, there is little known in terms of verified PPIs for the listed organisms, cross-species PPI predictions (as was done in [225], described in Section 5.1) will be done to create the predicted PPI networks for the four non-cerevisiae strains. As was seen in [225], PIPE appears to be biased towards making more accurate predictions for an organism when using its own known PPIs as its knowledge base as opposed to making cross-species predictions. On top of this, when comparing the predicted interaction networks of these five closely related yeast strains, it is not known how much change should be expected to be seen in a given PPI network due simple mutation alone. Because of this it becomes difficult to quantify the meaningful change in PPI networks. As far back as the late 1960s it was proposed that many genetic mutations, due to the sheer number of them, must be neutral in terms of overall effect and therefore an appropriate null hypothesis is crucial in evolutionary studies [135]. Null models are commonly used in sequence-based evolutionary studies [136, 193, 299], however the lack of well defined null models for network evolution has created controversy [63, 68, 91] over recent ENCODE [273] related publications.

The data presented in this section is currently undergoing final analysis and a manuscript describing the work has been prepared and is nearly completed. It is expected that this manuscript will be submitted for publication before the end of 
2015. Biological motivation and analysis presented here courtesy of collaborators Dr. Alex Wong, Dan Burnside and Houman Moteshareie from the Department of Biology at Carleton University.

\subsubsection{Wild Type Data}

For this project an updated set of 74,608 confirmed physical S. cerevisiae PPIs were collected from BioGRID [37]. These interactions will act as the PIPE database of known interactions for all predictions made across all real (wild type) as well as simulated strains studied. Scannell et al published improved, high quality genome sequences for S. bayanus, S. kudriavzevii, and S. mikatae and also provided highquality alignments of the genomes with better studied $S$. paradoxus and S. cerevisiae. In doing this, they identified a set of orthologous protein-coding genes in all 5 organisms [249]. The intersection of this set of orthologs with the genes which code for an updated set of 6,707 protein sequences collected from from UniProt [274] was taken, leaving 4,179 strict orthologs existing in all five organisms. All-against-all PPI predictions were made for this set of these orthologs in all five of the yeast strains listed above. The details of this computational task will be given in the next section. The phylogenetic tree for the five yeast strains studied here is given in Figure 6.5.

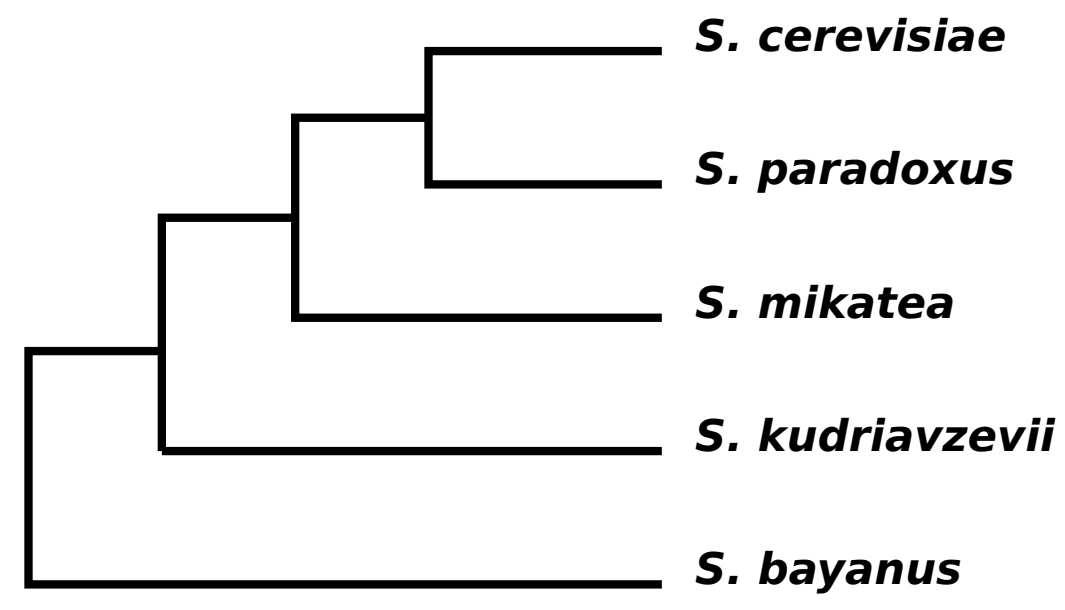

Figure 6.5: Phylogenetic tree for S. bayanus, S. kudriavzevii, S. mikatae, S. paradoxus and S. cerevisiae (reproduced from [249]) 


\subsubsection{Simulated Genomes \& the Construction of a Predicted PPI Network Null Model}

Using the sequence alignments supplied by Scannell et al, the number of mutations (nucleotide substitution) as well as the number and size of insertion-deletions (indels) across the five yeast strains could be inferred. To infer the nucleotide substitution parameters, the genes across all five strains were compared, one at a time, using PAML [299]. Essentially, this created a matrix containing all of the codons and their associated substitution probabilities observed across the five strains. For the indel operations, a simple parsimony model was used to determine the distribution of the number and size of the indels observed across the phylogenetic tree for each gene. This distribution was then used to estimate the parameters of a power-law function in $\mathrm{R}$ [231]. Using these inferred parameters for a given organism (as well as other inferred parameters relating to the length of the branches on the phylogenetic tree and the equilibrium frequencies of each codon), one could then generate a new set of gene sequences through simulated evolution using INDELible [81]. To do this for the non-cerevisiae strains, the $S$. cerevisiae genome sequence would be used as the starting point. As input INDELible would take this genome sequence as well as the mutation and indel parameters inferred above and the evolutionary distance between the input genome and the target genomes. The appropriate number of nucleotide substitutions and indel operations would then be made on each of the genes, simulating the evolution of the input genome to a new genomes that has the same evolutionary distance from $S$. cerevisiae as the target genomes. For example, what the above process does is start with the $S$. cerevisiae genome and apply the necessary mutations and indels to cover the evolutionary distance between $S$. cerevisiae and $S$. paradoxus in the phylogenetic tree (figure 6.5), thus creating a simulated S. paradoxus genome. From here the mutations continue to produce the simulated genomes of the other three species in the tree. S. cerevisiae is of course not the true root of the phylogenetic tree containing these five related organisms, but its use here as the starting point is justifiable given the use of reversible substitution models. This process was done 100 times for the entire phylogenetic tree, simulating the four noncerevisiae yeast organisms together in simulated evolution (starting at S. cerevisiae, evolving to a simulated $S$. paradoxus then to a simulated $S$. mikatae and so on). 
Doing it this way ensured that mutations would be shared between closely related organisms. This then resulted in 100 simulated genomes with the same evolutionary distance to the other wild type organisms as the real non-cerevisiae yeast strain in question. Once a simulated genome sequence was produced, the individual genes could then be translated into their respective protein sequence, resulting in 100 simulated proteomes for each of the four organisms. It should be noted that the locations of both point mutations and indels were random, it is only the observed rate and substitution probabilities of these mutations which is consistent with the wild type strains and are therefore random with respect to protein-protein interactions. This is equivalent to assuming that natural selection does not operate for or against mutations that modify PPIs, as is appropriate in a null model. Taken together, the collection of simulated proteomes will provide a baseline expectation for how many changes can be expected using the PIPE algorithm, given mutation but no natural selection.

MP-PIPE was run on all 400 simulated proteomes as well as the five wild type proteomes using HPCVL's Victoria Falls Cluster. The Victoria Falls Cluster is made up of 73 nodes interconnected by a gigabit switch. Each node has two UltraSparc T2+ chips $(1.2 \mathrm{Ghz})$ with 8 compute cores per chip. Each of these compute cores has 8 hardware supported threads giving each node a total of 128 hardware supported threads per node. Each node has either 64 GB RAM (10 nodes) or 32 GB RAM. The runs were performed using 33 nodes each with the MP-PIPE slave processes using 256 threads per node. Each of these runs made PPI predictions on the 8,734,110 individual protein pairs per proteome, for a total of 3,537,314,550 predictions overall. Since there were no/not enough known interaction data available for the four non-cerevisiae yeasts, the traditional LOOCV tests could not be performed. Because of this, it was decided to simply use the operating points determined through LOOCV tests done on the $S$. cerevisiae data. As these proteomes are actually a subset of the total number of true proteins that exist within each organism (as only the protein-coding genes which have orthologs across all 5 yeast organisms were taken) an operating point with a slightly lower specificity (99.5\%) was used. Using this as a cutoff, a set of protein pairs predicted to interact for each simulated or real proteome can be produced. Details about the size of the real (wild type) interactomes as well as the 100 simulated interactomes are summarized in Table 6.3. The predicted PPI networks 
for the $4 \times 100$ simulated strains would then make up the null model, as they have the same evolutionary characteristics as the wild type strains although randomly generated. Anything that appeared in the resulting PPI networks making up the null model represent phenomena that would be expected to appear simply at random and would not necessarily be selected for during the process of natural evolution. On the other hand, if phenomena within the predicted PPI networks of the wild type strains that do not occur within the null model is observed, these would be things that one would not expect to simply happen at random and would potentially occur due to selection pressures during evolution.

\begin{tabular}{lcccc}
\hline Strain & S. bayanus & S. kudriavzevii & S. mikatae & S. paradoxus \\
& & & & \\
Size of real interactome & 90,473 & 88,752 & 89,111 & 89,908 \\
$\begin{array}{l}\text { Avg size of simulated } \\
\text { interactome }\end{array}$ & $72,351.99$ & $74,069.29$ & $76,371.29$ & $81,741.31$ \\
$\begin{array}{l}\text { Min size of simulated } \\
\text { interactome }\end{array}$ & 71,549 & 73,082 & 75,226 & 80,682 \\
$\begin{array}{l}\text { Max size of simulated } \\
\text { interactome }\end{array}$ & 73,398 & 75,115 & 77,529 & 82,721 \\
$\begin{array}{l}\text { Median size of simulated } \\
\text { interactome }\end{array}$ & 72,292 & 74,047 & 76,371 & 81,751 \\
\end{tabular}

Table 6.3: Statistics on the size of the real (wild type) interactomes compared to the 100 simulated interactomes for each strain.

\subsubsection{Methods of Network Comparison}

Once the predicted PPI networks were completed for the five wild type yeast strains as well as the $4 \times 100$ simulated strains making up the predicted PPI network null model, the data was ready for analysis. 


\section{Sequence and Gene-centered Analysis}

Using the 4,179 orthologous proteins between the five species studies here, highly conserved genes as well as those genes which evolved relatively quickly were sought. Scannell et al. applied standard molecular evolutionary analyses implemented in PAML [299] to the full set of alignments. For each gene, an average rate of mutation parameter $(\omega)$ was estimated. This value is roughly the number of non-synonymous DNA substitutions (those substitutions which cause a change in the amino acid sequence of the protein the gene in question codes for) divided by the number of synonymous DNA substitutions (those substitutions which do not cause an amino acid change). The set of genes within the lowest tenth percentile of $\omega$ were taken as the set of slowly evolving (well conserved) genes. Naturally there were 418 proteins in this set. Scannell et al. provided further statistical analysis to identify those genes which evolved relatively fast compared to the rest. There were 123 rapidly evolving genes in this set.

These two sets of proteins represent those that would typically be studied using traditional protein and gene sequence based analysis. The results found by looking into these proteins will form the baseline results which be supplemented by the PPI and protein cluster analysis described below.

\section{Quantification of Changes in the PPI Network}

If a particular interaction is found to be absent in a single organism, the simplest assumption is that a single change (loss of interaction) has occurred along the phylogenetic tree. However, if an interaction is predicted to be absent in more than one organism in the tree, then it may be necessary to infer more than one change, depending on where in the phylogenetic tree these organisms are. To analyze this, the minimum number of changes (losses or gains of interaction) required to explain the observed pattern of presence/absence of each PPI predicted to be present in at least one organism was inferred by parsimony. To model this, a binary code of 5 bits for each interaction present in at least one of the five wild type organisms (1 means the interaction is present and 0 represents the interaction is missing in a given organism) can be assigned. Consider the following example with the phylogenetic tree in Figure 6.5 in mind. If a given interaction was present in all of the strains except $S$. cerevisiae 
and $S$. paradoxus, this would be given a code of 00111 which, in turn, represents that one loss of interaction occurred on the tree (on the branch just above where the branches for S. cerevisiae and S. paradoxus meet). This then leads to thirty-two possible state combinations for each interaction with at most three changes required for any given pattern. For a given interaction between proteins $i$ and $j$, the quantity $a_{i j}$ is defined as the number of predicted changes in interaction state for the pair across the phylogenetic tree. Since many proteins in the interaction network may have more than one interaction partner, for every protein $i$ with $n$ total interaction partners, the total number of changes in PPIs across the entire phylogenetic tree is calculated as:

$$
\gamma_{i}=\sum_{j=1}^{n} a_{i j}
$$

Using this definition, $\gamma_{i}$ is the total number of inferred changes across the entire phylogenetic tree for a given protein $i$.

Using the PIPE-predicted PPI networks, $\gamma_{i}$ was calculated for every protein $i$ across the phylogenetic tree for the wild type organisms, as well as in the 100 simulated phylogenies making up the null model. The distribution of $\gamma_{i}$ from the simulated phylogenies can be used as a null distribution to evaluate whether the $\gamma_{i}$ found across the wild type strains was unusually high or low with respect to this null model.

\section{Species-Specific Interaction Analysis}

Although a series of mutations of a gene (which leads to a series of protein-mutations) can lead to the addition or subtraction of an interaction, it is possible that the mutations behind this change of interaction were simply random events and not events that were selected for as a part of the evolutionary process. To identify the interactions that were selected for as a part of this process, one would need to exclude the interactions affected by meaningless, random changes. For each of the four non-cerevisiae yeast strains, interactions were identified that did not occur in any of their respective 100 simulated strains. These interactions could then be considered significant interactions that are not due to simple random mutation but must have been selected for as a part of the overall evolution process. Their absence in the simulated data suggests that they are easily disrupted by mutation. On top of these four sets of interactions 
(one for each wild type strain), a fifth set of interactions consisting of the intersection of these four sets as well as the interactions from S. cerevisiae was created. This set of interactions represents those that are conserved across all five wild type strains studied here but do not occur in any of the $4 \times 100$ simulated proteomes.

\section{Species-Specific Protein Cluster Analysis}

Dense sub-graphs of an interactome can represent functionally related proteins or proteins that interact to form protein clusters. OSLOM [148] is a computational tool used to find statistically significant clusters within a graph (described in Section 6.1). OSLOM was run on all of the predicted interactomes to give a set of overlapping clusters within each interactome. From here, clusters from different interactomes can be compared by counting how many proteins they have in common. A similarity score was defined between two clusters as the number of proteins the two clusters have in common divided by the size of the larger of the two clusters. Using this as a scoring function will always result in a score between 0 and 1 .

Similar to the species-specific interaction analysis, clusters that existed in the wild type strain but did not exist in any of the 100 simulated interactomes were sought. To determine if a given cluster was unique to the wild type strain, it was compared to all clusters in all 100 simulated interactomes. If it was determined not to match any of these clusters (here two clusters are considered a match if their similarity score is greater than or equal to 0.7) then it was deemed unique to the wild type strain. Again, similar to the interaction analysis, these clusters can be considered significant in that they are not a result of simple random mutation but must have been selected for as a part of the overall evolution process. GO term enrichment analysis was carried out on those clusters deemed to be unique to the wild type strain with respect to the simulated interactomes.

\section{Gene Ontology (GO) Analysis}

For sets of proteins, PPIs and protein complexes deemed noteworthy, GO term enrichment analysis was carried out. GO term enrichment analysis is typically carried out on a list of genes or proteins, however here enriched terms in a set of PPIs are of 
interest as well, so this conventional method of enrichment analysis had to be modified. For each interaction in a set of PPIs being analyzed, the GO terms that both proteins have in common are identified. Once this is done for every pair in the set, a set of GO terms common to some of the PPIs in the set as well as a count of how many times they were common to a pair in the given PPI set is left. Given this set of GO terms, a $p$-value could be calculated for each one given the number of times it was common to an interaction in the set, the total number of interactions in the set, the total number of possible interactions within the proteome and the number of these possible pairs that share the GO term in question. Traditional GO term enrichment analysis was carried out on the sets of conserved and rapidly evolving genes identified above as well as other notable sets of proteins and clusters identified. Specific rules were used to filter the GO enrichment results. In most cases, the following rules were applied:

- $p$-value less than 0.05

- must be a process GO term

- at least 3 of the proteins in the set must be associated with the tag (remove tags with only 1 or 2 associated proteins)

These rules were used in all cases with the exception of protein cluster analysis. Since clusters are much smaller sets of proteins compared to the other sets, the third rule was amended to allow for tags only associated with 2 proteins in the cluster. On top of this, for the cluster analysis, function GO terms were also investigated.

\subsubsection{Results}

The five closely related yeast strains, along with their respective 100 simulated strains acting as a null model, were analyzed using sequence-based, PPI-based and protein cluster-based methods. In each case elements which are highly conserved, rapidly evolving as well as those that exist in the wild type data but not in their respective simulations are highlighted to show the added value of analyzing PPI and protein clusters compared to strictly using sequence-based approaches. Over the next few sections two things will be highlighted. First, the fact that the new methods of analysis, when applied to both slowly and quickly evolving sets of proteins or PPIs, has overlap with the conventional methods of analysis lends support to the fact that 
these methods are producing quality results. Secondly, the novel results produced by these new methods represent data not previously attainable through conventional tools of analysis and could potentially be used to help explain the differentiation between the species studied here.

\section{Changes in PPIs Across the Phylogeny}

For each of the 4,179 othologous proteins, their respective $\gamma$ (which represents the total number of interaction changes, either losses or gains, over the entire phylogeny) was calculated as described. The mean, median and variance of the calculated $\gamma$ s were 3.0, 10.06 and 2001.74, respectively. The full distribution is given in Figure 6.6. From this figure it can be seen that most of the proteins are predicted to have very few PPI changes with a small number of proteins experiencing many changes. A protein's $\gamma$ is correlated with the number of interactions it participates in with the $S$. cerevisiae PPI network, meaning proteins with more interactions undergo more changes in interaction across the phylogeny (Figure 6.7). This correlation is not unexpected as a protein with more interactions has more opportunities to lose interactions than a protein with relatively few interactions. On the other hand, $\gamma$ is not correlated with overall rates of protein sequence evolution $(\omega)$ (Figure 6.8). It is likely that predicted changes in PPIs are mediated by substitutions in small motifs of a given protein. PIPE infers PPIs on the basis of short motifs which often represent only a very small subsection of a protein sequence. The smallest possible motif of 20 amino acids only covers a small fraction of a whole protein. PIPE exploits these motifs, which mediate PPIs, to make its predictions and thus amino acid changes outside of these predicted interaction sites will likely not have an enormous impact on the overall prediction score.

\section{Comparison of Molecular Evolution Using Sequence and PPI-based Methods}

In this set of comparisons, the sets of proteins which were well conserved and rapidly evolving at the sequence level were compared to those proteins which were well conserved and rapidly evolving at the PPI level. To determine if a protein's interactions were well conserved or highly evolving, its $\gamma$ value was used in conjunction with the 


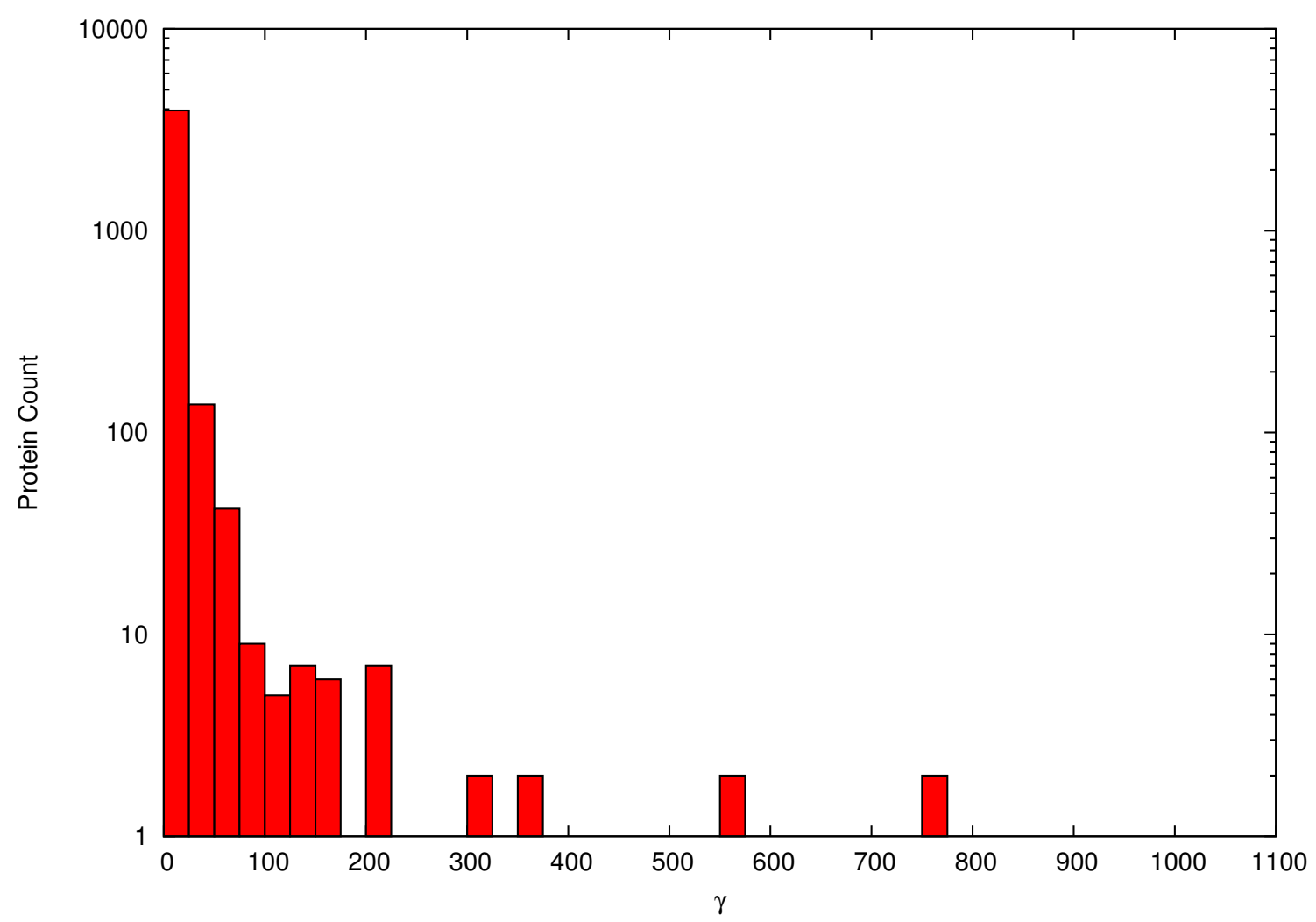

Figure 6.6: Histogram of $\gamma$ values for all 4,179 orthologous proteins across the 5 yeast strains. $\gamma$ represents the total number of interaction changes, either losses or gains, over the entire phylogenetic tree.

generated null distribution of $\gamma$. For each of the simulated phylogenies, each protein's respective $\gamma$ value was calculated. To find those proteins whose interactions are well conserved/highly evolving, those proteins which had a $\gamma$ below/above the range of $\gamma_{\mathrm{s}}$ witnessed in the simulations were identified. These represent proteins which have undergone less/more change in PPIs that would be expected at random. A $\gamma$ value below the range of $\gamma_{\mathrm{S}}$ in the simulated data sets provides evidence that natural selection has maintained PPIs by selecting against interaction-altering mutations whereas a $\gamma$ value above the range of $\gamma_{\mathrm{s}}$ in the simulated data represents a protein which has undergone a drastic change in PPI, at a rate higher than would be expected. 936 proteins with well conserved interactions and 191 proteins with rapidly evolving interactions were identified. The $\gamma$ values for these proteins, as well as the range of $\gamma_{\mathrm{s}}$ observed in the simulated data, are shown in Figures 6.9 and 6.10, respectively.

It is not surprising to see so many proteins which are expected to conserve their 


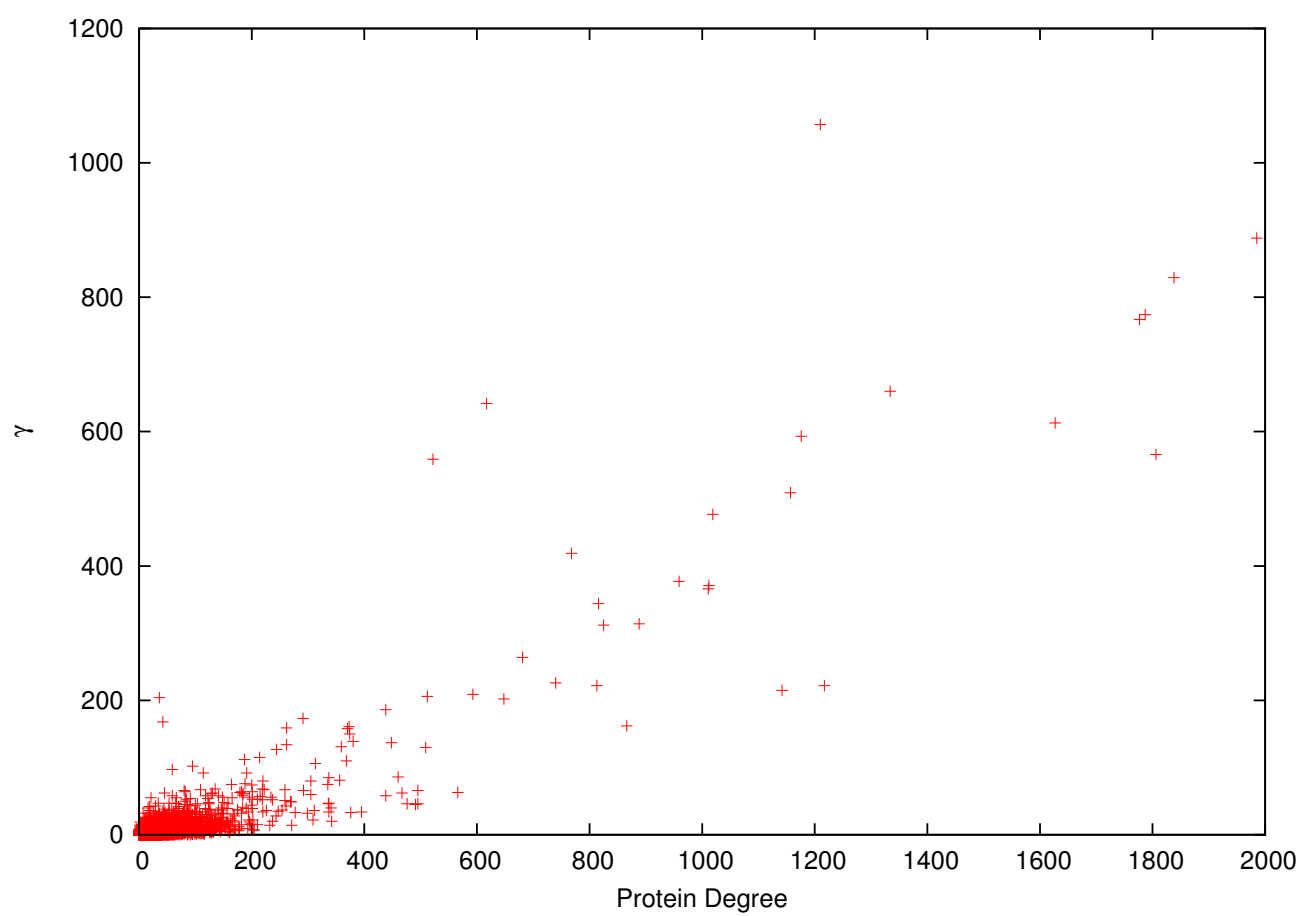

Figure 6.7: Comparing a protein's $\gamma$ value to its degree in the $S$. cerevisiae PPI network. $\gamma$ represents the total number of interaction changes, either losses or gains, over the entire phylogenetic tree.

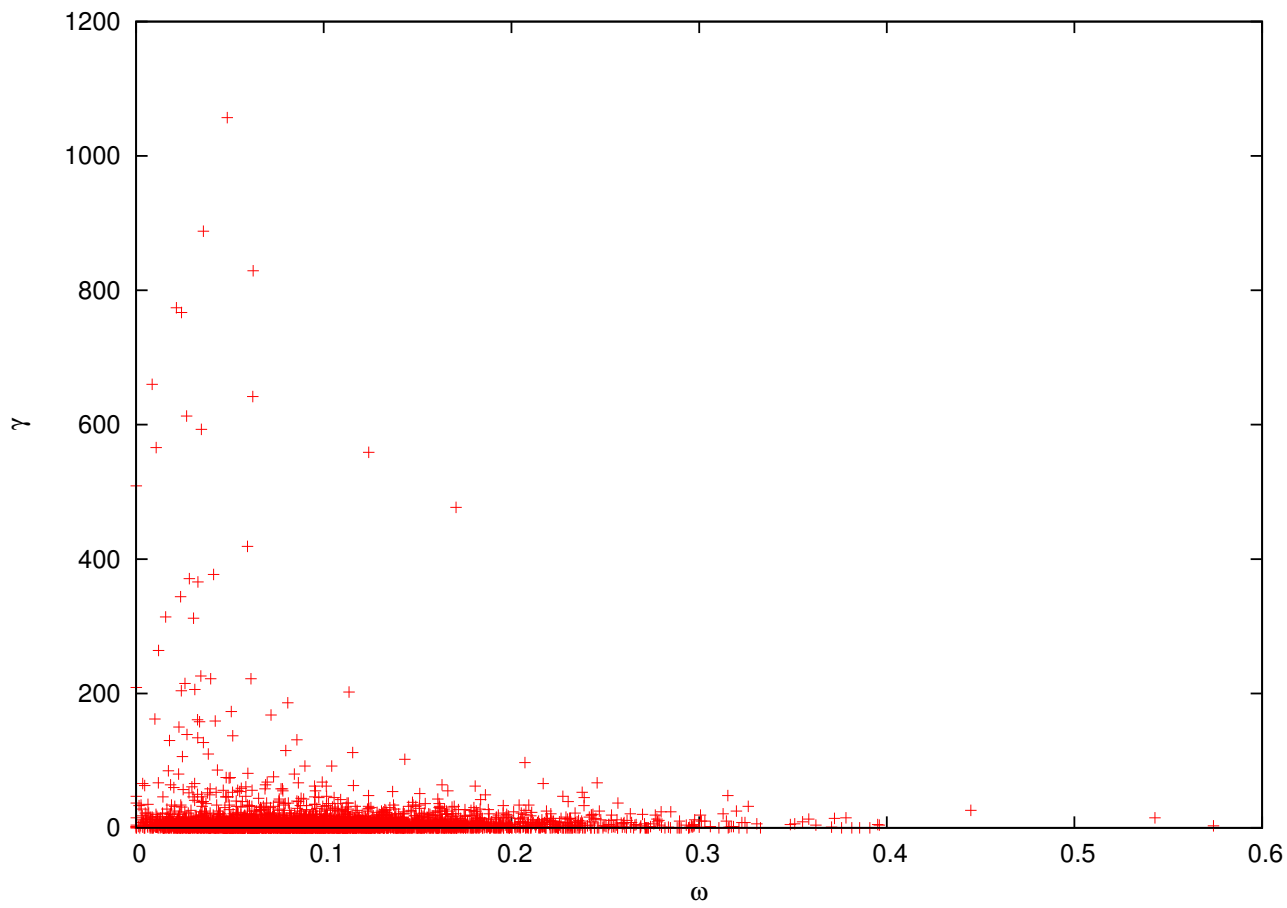

Figure 6.8: Comparing a protein's $\gamma$ value to its $\omega$ value. $\gamma$ represents the total number of interaction changes, either losses or gains, over the entire phylogenetic tree whereas $\omega$ is an estimation of the mutation rate (roughly the ratio of non-synonymous to synonymous DNA substitutions). 


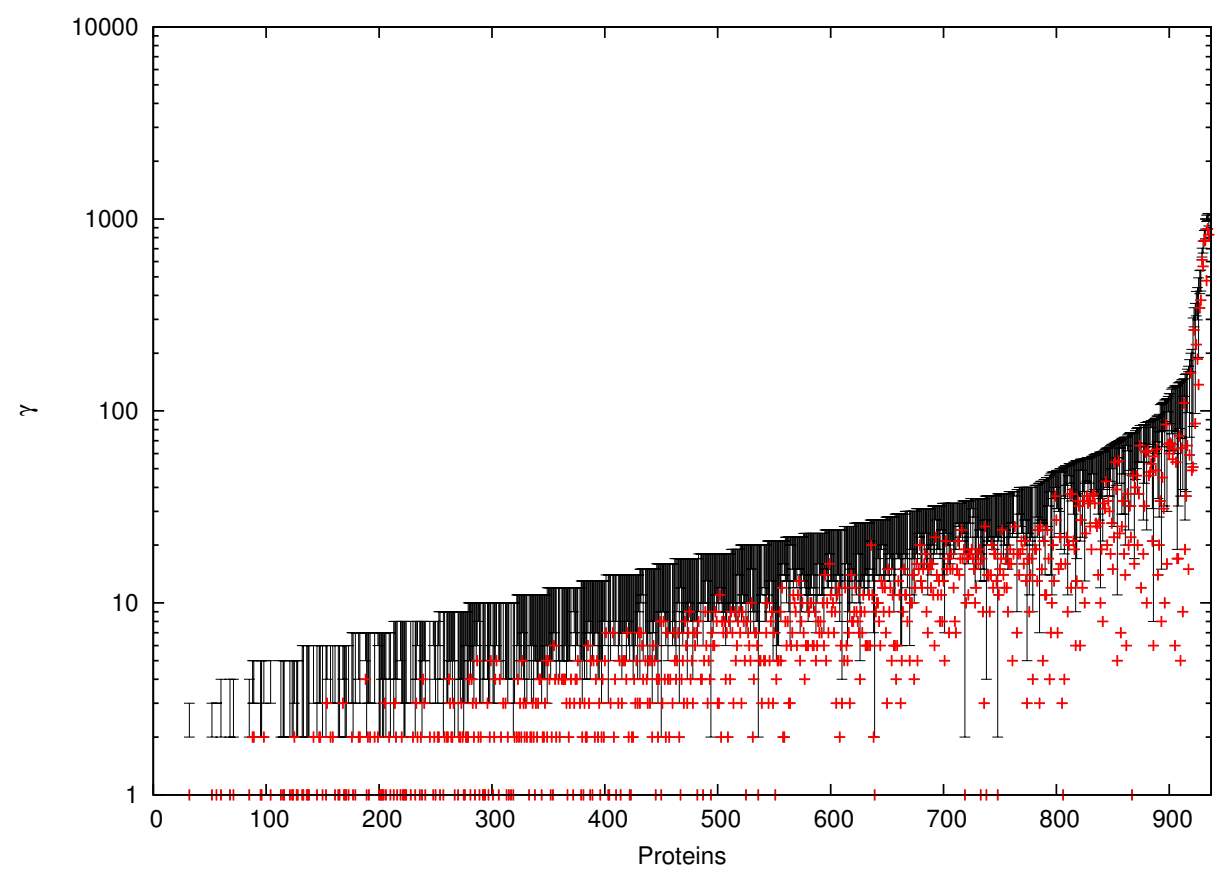

Figure 6.9: The $\gamma$ value of each protein identified as having well conserved interactions (red) with the respective range of $\gamma$ s observed for that protein in the simulated phylogenies (black). The proteins are sorted by the highest observed $\gamma$ value in the simulated phylogenies. The $\gamma$ axis is presented in logarithmic scale for readability.

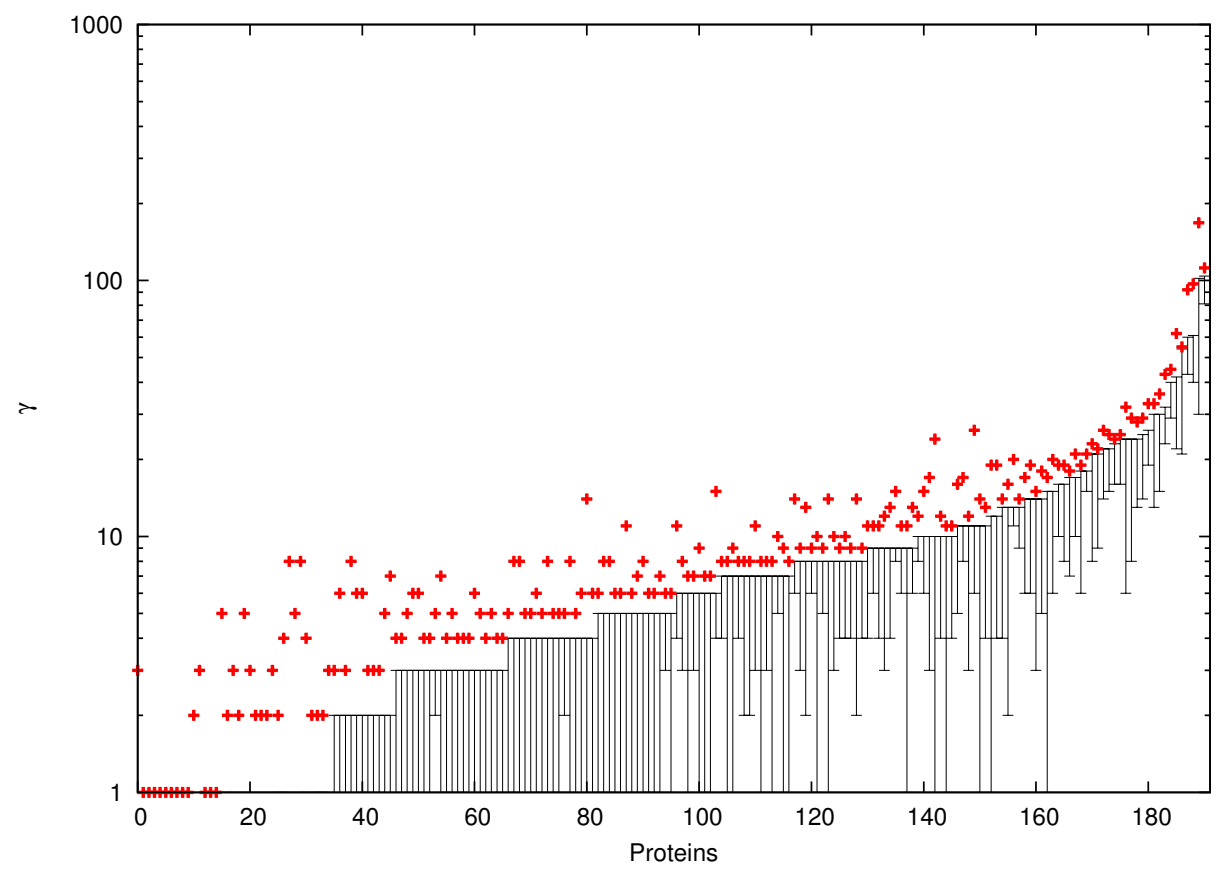

Figure 6.10: The $\gamma$ value of each protein identified as having well conserved interactions (red) with the respective range of $\gamma$ s observed for that protein in the simulated phylogenies (black). The proteins are sorted by the highest observed $\gamma$ value in the simulated phylogenies. The $\gamma$ axis is presented in logarithmic scale for readability. 
interactions as multiple PPI networks have been reported as being extremely conserved across both closely and distantly related phylogenies [29, 30, 83, 218]. The bulk of currently available data on conserved PPI networks comes from some of the most highly studied model organisms and does not deal with closely related species as the analysis here provides.

When comparing those proteins with highly conserved sequences to those with highly conserved PPIs across the phylogeny, an overlap of 108 proteins, which is slightly larger than expected by chance (based on one-sided Fisher's exact test, $p=$ 0.047), can be seen. On the other hand, the proteins with highly evolving sequences and PPIs had 9 common to both set, an overlap that is not different than what would be expected by chance (based on one-sided Fisher's exact test, $p=0.107$ ). A summary of this comparison as well as a comparison of their enriched GO process terms are given in Table 6.4.

\begin{tabular}{lcc}
\hline Protein Set & \# Proteins & \# Enriched GO Terms \\
\hline & 418 & 87 \\
Conserved Sequences & 936 & 101 \\
Lower Than Expected $\gamma$ & 108 & 15 \\
Overlap & & \\
& & 7 \\
Rapidly Evolving Sequences & 123 & 12 \\
Higher Than Expected $\gamma$ & 191 & 1 \\
Overlap & 9 & \\
\hline
\end{tabular}

Table 6.4: Comparison between the GO term enrichment for sets of conserved and highly evolving proteins determined with sequence based and PPI-based methods.

The overlap here shows that the proteins with higher or lower than expected $\gamma$ are enriched for some of the same GO terms as identified via sequence-based methods. The overlapping GO term from the quickly evolving sets was GO:0006810 (transport). 16 of the high $\gamma$ proteins and 11 of the quickly evolving protein sequences were associated with this GO term. The overlapping terms for the slowly evolving sets are given in Table 6.5.

On the other hand however, the non-overlapping results represent those that are 


\begin{tabular}{|c|c|c|c|}
\hline GO Term ID & GO Term Name & $\begin{array}{l}\# \text { Low } \gamma \\
\text { proteins }\end{array}$ & $\begin{array}{l}\text { \# Slowly Evolving } \\
\text { proteins }\end{array}$ \\
\hline GO:0015031 & protein transport & 94 & 46 \\
\hline GO:0042254 & ribosome biogenesis & 48 & 23 \\
\hline GO:0006888 & $\begin{array}{l}\text { ER to Golgi vesicle-mediated } \\
\text { transport }\end{array}$ & 25 & 13 \\
\hline GO:0000462 & $\begin{array}{l}\text { maturation of SSU-rRNA from } \\
\text { tricistronic rRNA transcript }\end{array}$ & 11 & 4 \\
\hline GO:0006616 & $\begin{array}{l}\text { SRP-dependent cotranslational } \\
\text { protein targeting to membrane } \\
\text { \& translocation }\end{array}$ & 4 & 4 \\
\hline GO:0006886 & intracellular protein transport & 25 & 16 \\
\hline GO:0010499 & $\begin{array}{l}\text { proteasomal ubiquitin-independent } \\
\text { protein catabolic process }\end{array}$ & 6 & 7 \\
\hline GO:0006359 & $\begin{array}{l}\text { regulation of transcription from } \\
\text { RNA polymerase III promoter }\end{array}$ & 3 & 3 \\
\hline GO:0055085 & transmembrane transport & 41 & 8 \\
\hline GO:0016192 & vesicle-mediated transport & 38 & 24 \\
\hline GO:0006950 & response to stress & 29 & 17 \\
\hline GO:0002181 & cytoplasmic translation & 40 & 29 \\
\hline GO:0006364 & rRNA processing & 54 & 29 \\
\hline GO:0008152 & metabolic process & 31 & 38 \\
\hline GO:0007264 & $\begin{array}{l}\text { small GTPase mediated signal } \\
\text { transduction }\end{array}$ & 22 & 15 \\
\hline
\end{tabular}

Table 6.5: The intersection of enriched GO terms for proteins with lower than expected $\gamma$ and proteins with slowly evolving sequences.

provided only by the newly introduced methods of analysis, showing that analyzing the $\gamma$ of a protein, and finding those proteins which had a higher or lower than expected $\gamma$, adds additional information to the comparison of these species. The GO terms for which the proteins with higher than expected $\gamma$ values were enriched for that were not identified using the sequence based analysis are given in Table 6.6. The entire set of unique GO terms for the respective low $\gamma /$ slowly evolving proteins are omitted due to the size of the data set, however the ten GO terms with the lowest $p$-value are given in Table 6.7. 
GO Term ID GO Term Name

\# High $\gamma$ proteins $p$-value

\begin{tabular}{|c|c|c|c|}
\hline GO:0008152 & metabolic process & 3 & 0.0020 \\
\hline GO:0006351 & transcription, DNA-dependent & 28 & 0.0079 \\
\hline GO:0010526 & $\begin{array}{l}\text { negative regulation of transposition, } \\
\text { RNA-mediated }\end{array}$ & 3 & 0.0029 \\
\hline GO:0006355 & $\begin{array}{l}\text { regulation of transcription, } \\
\text { DNA-dependent }\end{array}$ & 28 & 0.0035 \\
\hline GO:0006357 & $\begin{array}{l}\text { regulation of transcription from RNA } \\
\text { polymerase II promoter }\end{array}$ & 10 & 0.0042 \\
\hline GO:0016310 & phosphorylation & 3 & 0.0168 \\
\hline GO:0015031 & protein transport & 9 & 0.0393 \\
\hline GO:0055085 & transmembrane transport & 3 & 0.0406 \\
\hline GO:0000122 & $\begin{array}{l}\text { negative regulation of transcription } \\
\text { from RNA polymerase II promoter }\end{array}$ & 8 & 0.0044 \\
\hline GO:0006366 & $\begin{array}{l}\text { transcription from RNA polymerase } \\
\text { II promoter }\end{array}$ & 8 & 0.0186 \\
\hline GO:0006397 & mRNA processing & 12 & 0.0217 \\
\hline
\end{tabular}

Table 6.6: Enriched GO terms for proteins with higher than expected $\gamma$ not identified when analyzing highly evolving protein sequences.

As can be seen in Table 6.7, proteins with lower than expected $\gamma$ values are enriched for proteins involved in phosphorylation (GO:0016310, $p$-value $1.24 \times 10^{-7}$ and GO:0006468, $p$-value $4.34 \times 10^{-12}$. Of the 164 and 106 proteins associated with these GO terms, 66 and 56 were identified here, respectively. It is well known that the family of kinase proteins (involved in phosphorylation) contain highly conserved regions of residues essential to their function [71]. Using conserved features, the phylogenetic mapping of catalytic domains has been done to successfully characterize newly identified kinases [102]. The activation of protein kinases (a kinase protein acquiring a phosphate group to use for phosphorylation) also appears to be highly conserved. Known domains such as crystathione $\beta$-synthase (CBS or Bateman domains) are conserved across distant eukaryotic lineages and are found in a large variety of enzymes. These domains contain regions that are key to the acquisition of phosphate groups (ATP/AMP binding residues). It has been shown that mutations in these regions have detrimental effects in both yeast and human enzymes and have been linked to 


\begin{tabular}{llcc} 
GO Term ID & GO Term Name & \# Low $\gamma$ proteins & $p$-value \\
& & & \\
\hline & & 56 & $4.34 \times 10^{-12}$ \\
GO:0006468 & protein phosphorylation & 66 & $1.24 \times 10^{-7}$ \\
GO:0016310 & phosphorylation & 3 & $4.72 \times 10^{-7}$ \\
GO:0032543 & mitochondrial translation & 40 & $1.52 \times 10^{-5}$ \\
GO:0002181 & cytoplasmic translation & 175 & $2.38 \times 10^{-5}$ \\
GO:0006810 & transport & 29 & $3.57 \times 10^{-5}$ \\
GO:0006897 & endocytosis & 22 & $1.17 \times 10^{-4}$ \\
GO:0007264 & small GTPase mediated & 14 & $1.45 \times 10^{-4}$ \\
& signal transduction & 12 & $1.54 \times 10^{-4}$ \\
GO:0019236 & response to pheromone & & \\
GO:0003333 & amino acid transmembrane & 10 & $2.99 \times 10^{-4}$ \\
& transport & & \\
\hline GO:0006913 & nucleocytoplasmic transport & & \\
\hline
\end{tabular}

Table 6.7: Enriched GO terms for proteins with lower than expected $\gamma$ not identified when analyzing highly evolving protein sequences. Of the 86 unique GO terms identified, the 10 with the lowest $p$-value are displayed.

multiple disease states $[105,256]$.

Yeast plays an important role in wine production and, as a result, wine producers seek yeast strains with certain qualities. Low temperature fermentation allows for more consistent and efficient fermentation while also improving the overall sensory quality of the final product. Yeast strains that are tolerant to low temperature prefermentation soaks [178] or are able to ferment wine efficiently at low temperatures [49] are of particular interest to wine producers [56]. Global metabolic analysis has suggested that $S$. bayanus and $S$. kudriavzevii employ unique strategies for cold tolerance. S. bayanus does this by upregulating shikimate aromatic amino acid biosynthesis whereas S. kudriavzevii has a higher Nad+ production [166]. At low temperatures the transportation of aromatic amino acids is impaired due to mechanical stress caused by increased membrane rigidity. In response to this in S. cerevisiae, tryptophan transporter Tat2p among others are over-expressed [2]. Proteins with lower than expected $\gamma$ are enriched for the term GO:0003333, amino acid transmembrane transport ( $p$-value $1.54 \times 10^{-4}$ ), meaning it is predicted that this functionality is conserved across the five species studied here. This suggests that this conserved 
functionality could potentially play a role in $S$. bayanus and $S$. kudriavzevii cold tolerance.

The fact that the proteins predicted to have significantly fewer changes of interactions are enriched for properties that are known to be well conserved adds credibility to the novel data presented through this analysis.

\section{PPI-based Analysis}

In order to find PPIs which may be particularly interesting within each species, those interactions that are present in the predicted PPI network of the wild type data while being absent from the respective 100 simulated strains were sought. These interactions represent those that would easily be lost due to random mutation and are thus likely to be rejected by natural selection. These interactions are therefore predicted to be easily interrupted and this suggests that purifying selection maintains the interaction within the living species. To find these interactions, each set of PPIs within a given wild type strain was filtered to remove any interactions that occurred within the respective 100 simulated strains. The number of such interactions in each species are as follows: S. bayanus - 7,552, S. kudriavzevii - 4,902, S. mikatae - 3,894 and S. paradoxus - 1,779. A fifth set of interactions were also gathered which consisted of the intersection of the previously identified interactions as well as those predicted in the $S$. cerevisiae. These interactions represent those that are completely conserved across all five yeast strains while also not occurring in any of the $4 \times 100$ simulated strains. There were 662 interactions in this set.

GO term enrichment analysis was done on the 662 conserved, significant interactions identified above. The results from this analysis can naturally be compared to the GO enrichment analysis performed on the proteins which were highly conserved at the sequence level. As seen previously, there were 418 proteins in the set of highly conserved sequences and they were enriched for 87 GO terms. The set of 662 conserved interactions were enriched for 22 GO terms, none of which were common to the highly conserved sequence set. These enriched GO terms are listed in Table 6.8

GO term enrichment analysis was also carried out on the individual, speciesspecific sets of significant interactions. On top of this, the results of GO term enrichment analysis for each species was then filtered to identify those GO terms that 


\begin{tabular}{|c|c|c|c|}
\hline GO Term ID & GO Term Name & $\begin{array}{l}\text { \# Significant } \\
\text { Conserved PPIs }\end{array}$ & $p$-value \\
\hline GO:0006696 & ergosterol biosynthetic process & 10 & $5.63 \times 10^{-25}$ \\
\hline GO:0006694 & steroid biosynthetic process & 10 & $1.42 \times 10^{-24}$ \\
\hline GO:0000750 & $\begin{array}{l}\text { pheromone-dependent } \\
\text { signal transduction }\end{array}$ & 7 & $2.18 \times 10^{-17}$ \\
\hline GO:0006629 & lipid metabolic process & 13 & $1.49 \times 10^{-13}$ \\
\hline GO:0030261 & chromosome condensation & 4 & $6.69 \times 10^{-13}$ \\
\hline GO:0000070 & $\begin{array}{l}\text { mitotic sister chromatid } \\
\text { segregation }\end{array}$ & 5 & $2.01 \times 10^{-11}$ \\
\hline GO:0007067 & mitosis & 10 & $2.16 \times 10^{-11}$ \\
\hline GO:0007165 & signal transduction & 7 & $6.48 \times 10^{-11}$ \\
\hline GO:0051301 & cell division & 12 & $2.15 \times 10^{-10}$ \\
\hline GO:0007186 & $\begin{array}{l}\text { G-protein coupled receptor } \\
\text { signalling pathway }\end{array}$ & 3 & $5.76 \times 10^{-10}$ \\
\hline GO:0070058 & tRNA gene clustering & 3 & $5.76 \times 10^{-10}$ \\
\hline GO:0007076 & mitotic chromosome condensation & 3 & $6.14 \times 10^{-9}$ \\
\hline GO:0019236 & response to pheromone & 4 & $1.06 \times 10^{-8}$ \\
\hline GO:0031684 & $\begin{array}{l}\text { heterotrimeric G-protein } \\
\text { complex cycle }\end{array}$ & 2 & $8.60 \times 10^{-8}$ \\
\hline GO:0051276 & chromosome organization & 3 & $1.21 \times 10^{-7}$ \\
\hline GO:0007049 & cell cycle & 13 & $1.81 \times 10^{-6}$ \\
\hline GO:0007062 & sister chromatid cohesion & 2 & $2.17 \times 10^{-6}$ \\
\hline GO:0006409 & tRNA export from nucleus & 2 & $3.61 \times 10^{-6}$ \\
\hline GO:0006607 & $\begin{array}{l}\text { NLS-bearing substrate import } \\
\text { into nucleus }\end{array}$ & 2 & $3.61 \times 10^{-6}$ \\
\hline GO:0005975 & carbohydrate metabolic process & 4 & $1.55 \times 10^{-5}$ \\
\hline GO:0006098 & pentose-phosphate shunt & 2 & $2.34 \times 10^{-5}$ \\
\hline GO:0006310 & DNA recombination & 2 & $9.84 \times 10^{-4}$ \\
\hline
\end{tabular}

Table 6.8: Enriched GO terms for proteins participating in conserved, significant PPIs. This set of PPIs are conserved across all five species studied (including $S$. cerevisiae) while also not appearing in any of the $4 \times 100$ simulated interactomes. 
were unique to that specific species. This would then mean that a portion of those interactions predicted to be protected by purifying selection would be enriched for unique GO terms. A summary of these results are given in Table 6.9. Table 6.10 lists the unique GO terms associated with the significant interactions in each species. The interactions which were predicted in $S$. bayanus but were not predicted in any of the other wild type or simulated strains were enriched for GO term GO:0001403, invasive growth in response to glucose limitation $\left(p\right.$-value $\left.3.04 \times 10^{-5}\right)$. This is consistent with a previous finding that showed that in $S$. cerevisiae galactose metabolism genes can only be induced by the presence of galactose but in $S$. bayanus they are also induced in response to less preferred carbon sources such as ethanol, raffinose, sucrose and glycerol [31]. Taken together, this suggests that the unique vitality of S. bayanus under glucose limitation could involve both the up-regulation of galactose metabolism genes and the maintenance of PPIs involved in invasive growth.

\section{Protein Cluster-based Analysis}

To extend the analysis performed in the previous section, potentially novel and significant protein complexes were sought. The previous sections have provided individual protein and PPI-level analysis but higher levels of organization (including protein complexes) may also be important for evolution. Protein complexes that are potentially important to each lineage were identified in a similar manner to the previous section. Interesting clusters were identified as those that occur in the wild type strain but were absent from all of the simulated interactomes. GO term enrichment analysis was carried out on each individual protein cluster identified. Enriched GO terms that were unique to a given species' set of significant clusters were then collected. This analysis is summarized in Table 6.11.

One of the significant clusters identified in S. bayanus was uniquely enriched for the go term GO:0045039, protein import into mitochondrial inner membrane ( $p$-value $\left.9.33 \times 10^{-17}\right)$. Half of the 14 proteins that make up this cluster are associated with this GO term, while only 3 other yeast proteins outside of this cluster are associated with this term. Included in this cluster are the key members of this function. A group of proteins involved in this complex are previously identified as translocase proteins of the inner membrane. Translocase proteins Tim9 and Tim10 assist carriers across the 


\begin{tabular}{lcccc}
\hline Species & $\begin{array}{c}\text { \# Significant } \\
\text { PPIs }\end{array}$ & $\begin{array}{c}\text { \# Unique } \\
\text { Significant PPIs }\end{array}$ & $\begin{array}{c}\text { \# Enriched } \\
\text { GO Terms }\end{array}$ & $\begin{array}{c}\text { \# Unique Enriched } \\
\text { GO Terms }\end{array}$ \\
\hline S. bayanus & 7,552 & 3,204 & 71 & 20 \\
S. kudriavzevii & 4,902 & 1,068 & 21 & 2 \\
S. mikatae & 3,894 & 798 & 17 & 1 \\
S. paradoxus & 1,779 & 709 & 7 & \\
\hline
\end{tabular}

Table 6.9: Comparing the significant PPIs and their associated GO term enrichment between the four non-cerevisiae species. A significant PPI is defined as a PPI occurring in the wild type data but in none of the 100 respective simulations. These were filtered to find the unique significant PPIs for each species. GO enrichment analysis was carried out on the unique significant PPIs and then unique GO terms were identified for each set of PPIs.

\begin{tabular}{|c|c|c|c|}
\hline Species & GO Term ID & GO Term Name & $p$-value \\
\hline S. bayanus & GO:0006897 & endocytosis & $8.95 \times 10^{-9}$ \\
\hline S. bayanus & GO:0006342 & chromatin silencing & $1.60 \times 10^{-7}$ \\
\hline S. bayanus & GO:0001403 & $\begin{array}{l}\text { invasive growth in response } \\
\text { to glucose limitation }\end{array}$ & $3.04 \times 10^{-5}$ \\
\hline S. bayanus & GO:0035269 & $\begin{array}{l}\text { protein O-linked } \\
\text { mannosylation }\end{array}$ & $5.05 \times 10^{-5}$ \\
\hline S. bayanus & GO:0000462 & $\begin{array}{l}\text { maturation of SSU-rRNA } \\
\text { from tricistronic rRNA transcript }\end{array}$ & $5.82 \times 10^{-5}$ \\
\hline S. kudriavzevii & GO:0016192 & vesicle-mediated transport & $8.07 \times 10^{-3}$ \\
\hline S. kudriavzevii & GO:0008380 & RNA splicing & $7.70 \times 10^{-3}$ \\
\hline S. mikatae & GO:0000070 & $\begin{array}{l}\text { mitotic sister chromatid } \\
\text { segregation }\end{array}$ & $2.62 \times 10^{-4}$ \\
\hline S. mikatae & GO:0006351 & transcription, DNA-dependent & $2.06 \times 10^{-2}$ \\
\hline S. paradoxus & GO:0000723 & telomere maintenance & $1.14 \times 10^{-4}$ \\
\hline
\end{tabular}

Table 6.10: Unique enriched GO terms for the significant PPIs. Significant PPIs for each species were filtered to find the species-specific significant interactions. GO term enrichment analysis was performed to identify the unique terms associated with each set of PPIs. The 5 GO terms with the lowest $p$-value for $S$. bayanus of the 20 identified are presented. 


$\begin{array}{cccc}\text { Species } & \text { \# Significant Protein } & \text { \# Clusters with } & \text { \# Clusters with } \\ \text { Clusters } & \text { Enriched GO Terms } & \text { Unique GO Terms }\end{array}$

$\begin{array}{llll}\text { S. bayanus } & 27 & 27 & 21 \\ \text { S. kudriavzevii } & 22 & 21 & 21 \\ \text { S. mikatae } & 14 & 14 & 13 \\ \text { S. paradoxus } & 14 & 14 & 13\end{array}$

Table 6.11: Significant protein clusters and GO term enrichment analysis results found in each of the four non-cerevisaie species. A significant cluster is defined as one that occurs in the wild type interactome but in none of the 100 simulated interactomes for a given species.

intermembrane space and dock with Tim12. The protein insertion complex (Tim22 complex) comprises Tim22, Tim18, Tim 54, and Sdh3 and functions to weave proteins through the inner membrane. The significant cluster in question includes all 7 of these proteins, despite no significant similarity between their protein sequences. Also included in the complex are the key members of the succinate dehydrogenase enzyme, the only enzyme which participates in both of the citric acid cycle and the electron transport chain. This suggests that in the evolution of S. bayanus the interaction network between this set of essential inner mitochondrial membrane proteins has been specifically selected for, potentially because the arrangement is important for inserting inter-membrane proteins (eg. succinate dehydrogenase) in this species. This cluster, including the predicted PPIs between the cluster members, is visualized in Figure 6.11.

Another example of novel data produced through this analysis which can be tied to previous knowledge comes from the $S$. kudriavzevii predicted PPI network. S. $k u$ driavzevii is known to be extremely sensitive to ethanol, more so than any of the other species studied here [17]. The genes most envolved with alcohol tolerance are associated mainly with cytoskeleton organization, biogenesis and transport (particularly involving the vacuole, peroxisome and endosome) [271]. Unique GO terms associated with clusters only found in S. kudriavzevii may help explain this hyper-sensitivity to ethanol. One cluster in S. kudriavzevii is uniquely enriched for GO terms associated 


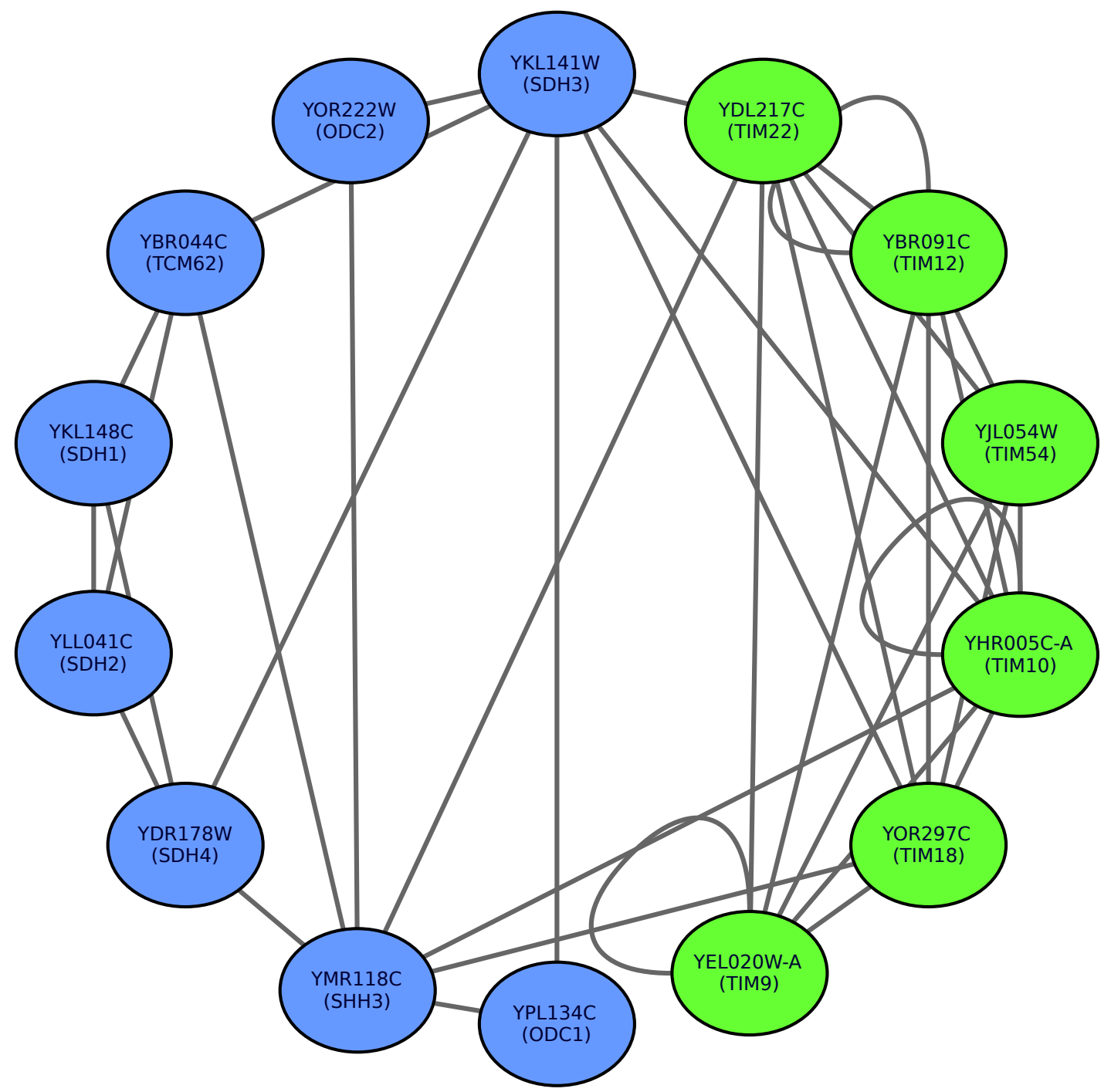

Figure 6.11: Significant cluster found within the S. bayanus predicted PPI network. This cluster contains translocase proteins which participate in the known protein insertion complex (Tim22). These Tim proteins are coloured in green.

with cytoskeleton organization, biogenesis as well as the specific GO term late endosome to vacuole transport. The GO terms associated with this cluster are summarized in Table 6.12. Other identified clusters were enriched for GO terms GO:0004026 alcohol O-acetyltransferase activity ( $p$-value $1.14 \times 10^{-4}$ ) and GO:0030242 peroxisome degradation $\left(p\right.$-value $\left.3.0 \times 10^{-5}\right)$. It is possible that these unique interaction patterns in S. kudriavzevii (which are enriched for terms associated with processes most important for alcohol tolerance) help to explain this unique phenotype. 


\begin{tabular}{|c|c|c|c|}
\hline GO Term ID & GO Term Name & \# Cluster proteins & $p$-value \\
\hline GO:0030472 & $\begin{array}{l}\text { mitotic spindle organization } \\
\text { in nucleus }\end{array}$ & 6 & $5.01 \times 10^{-07}$ \\
\hline GO:0007059 & chromosome segregation & 9 & $6.71 \times 10^{-07}$ \\
\hline GO:0045324 & $\begin{array}{l}\text { late endosome to vacuole } \\
\text { transport }\end{array}$ & 5 & $3.28 \times 10^{-06}$ \\
\hline GO:0005200 & $\begin{array}{l}\text { structural constituent of } \\
\text { cytoskeleton }\end{array}$ & 6 & $5.52 \times 10^{-06}$ \\
\hline GO:0031110 & $\begin{array}{l}\text { regulation of microtubule } \\
\text { polymerization or } \\
\text { depolymerization }\end{array}$ & 4 & $1.25 \times 10^{-05}$ \\
\hline GO:0008017 & microtubule binding & 5 & $2.33 \times 10^{-05}$ \\
\hline GO:0003777 & microtubule motor activity & 3 & $1.16 \times 10^{-04}$ \\
\hline GO:0030473 & $\begin{array}{l}\text { nuclear migration along } \\
\text { microtubule }\end{array}$ & 3 & $1.156 \times 10^{-04}$ \\
\hline GO:0007020 & microtubule nucleation & 4 & $1.64 \times 10^{-04}$ \\
\hline GO:0000132 & $\begin{array}{l}\text { establishment of mitotic } \\
\text { spindle orientation }\end{array}$ & 3 & $2.00 \times 10^{-04}$ \\
\hline GO:0000741 & karyogamy & 3 & $3.15 \times 10^{-04}$ \\
\hline GO:0008154 & $\begin{array}{l}\text { actin polymerization or } \\
\text { depolymerization }\end{array}$ & 2 & $3.35 \times 10^{-04}$ \\
\hline GO:0003786 & actin lateral binding & 2 & $3.35 \times 10^{-04}$ \\
\hline GO:0007119 & $\begin{array}{l}\text { budding cell isotropic } \\
\text { bud growth }\end{array}$ & 2 & $9.93 \times 10^{-04}$ \\
\hline
\end{tabular}

Table 6.12: Enriched GO terms for proteins making up a significant cluster in $S$. kudriavzevii. A significant cluster is defined as one that occurs in the wild type strain but does not occur in any of the simulated interactomes. GO terms presented are unique to clusters in S. kudriavzevii. 


\subsection{Conclusion}

Over the past few decades, the traditional methods of analysis used when comparing related species were generally sequenced-based and were used to compare the genome sequences of the organisms in question. While these methods have produced a wealth of knowledge, over the past two sections it has been shown that there is added value in also investigating the predicted PPI networks of the organisms as well. In Section 6.2, a family of related Volvocine algae were studied in hopes of uncovering some insights into their transition from unicellularity to multicellularity. Although the species involved show great differences at a high level, previous comparisons of their genomes didn't show dramatic differences. To add to the gene-specific analysis performed previously the predicted PPI networks of these organisms were compared, specifically looking for trends in terms of modularity and integration. Overall, the modularity measures identified increased while the measures of integration decreased during the transition from unicellularity to multicellularity. If these trends hold true in other species undergoing the same transition is left as an open question, however the analysis done certainly supplements what was currently known about these organisms.

In Section 6.3, five closely related yeast strains were studied. Here, the knowledge which could be attained through sequence-based methods of analysis was used as a baseline measure to see if using PPI or protein cluster based methods of analysis could provide additional insights. A novel null model was also introduced to attempt to differentiate those changes which were due to simple random mutation and those changes that were truly meaningful between the organisms. When analyzing aspects of the PPI networks which were well conserved or rapidly evolving, it was shown that the newly introduced methods of analysis produced an overlap with with the more traditional sequence-based methods. There was also a sizeable amount of novel data produced, showing the value in looking at PPI networks in this context as well. Through the use of the null model, significant aspects of the networks could be identified as those that occurred in nature but not in any of the simulations and were thus likely not to have just occurred at random. These aspects couldn't naturally be compared to the sequence-based methods applied and thus all of these results were novel. It is hoped that this data can be further analyzed to help explain some of the 
phenotypic differences between the species studied. 


\section{Chapter 7}

\section{Engineering Inhibitory Proteins with InSiPS: The In-Silico Protein Synthesizer}

\subsection{Introduction}

For nearly 30 years the task of creating new proteins with specific characteristics has been of great interest to the biological community. Protein engineering refers to a field of research which strives to build optimal proteins with specific functionalities. In a sense, the problem of protein engineering has classically been considered the reverse problem of protein function prediction. In function prediction, one is given the protein sequence and asked to approximate its three dimensional structure (protein folding) and then determine the interactions that protein participates in (protein docking), while in protein engineering a target function is decided upon and it is the designer's job to come up with a sequence to fit the targeted properties. The approaches to protein engineering can generally be divided into two camps. The first set of approaches aim to modify existing proteins to improve or amend a protein's function [48] and the second are de novo protein design approaches which aim to generate novel proteins optimized for specific functions [314].

Over the past few decades this field has expanded greatly and produced a wide variety of approaches (some reviewed in $[132,207,248,306]$ ). As far as 25 years ago, researchers were able to design relatively simple proteins fitting given structures to perform a specific functions [57]. The function of a protein $A$ is mediated by its interactions with other proteins, in other words a set of target and non-target proteins with which $A$ interacts and doesn't interact with, respectively [276]. Successfully designed proteins with specific targets and non-targets can be used in a regulatory fashion, enhancing or inhibiting the function of the targets. These regulatory proteins can also have the effect of altering the target protein's structure. The design of inhibitory proteins (proteins which bind specifically to a single target with high 
specificity) is of particular interest as these proteins can prevent the target protein from performing its normal function in the cell $[6,52]$. A protein critical to a known pathogen would make a natural target for a designed inhibitory protein (eg. an HIV protein [239]). Once the key protein is inhibited, the overall impact of the pathogen could potentially be reduced. Inhibitory proteins like this can form the basis for the development of various therapeutics [283].

Although a vast amount of progress has been made in the field of protein design, there exists some room for improvement. Recent computational approaches have had mixed results in terms of experimental validation, most of which aim to redesign naturally occurring protein folds rather than designing novel proteins ab initio [20]. The three-dimensional structure of the proteins often play a central role in protein design as proteins are typically designed to fit specific structures. Once these structures have been established, they then can carry out the desired function. The fact that structural information plays such a central role in these approaches leads to similar limitations that structure-based PPI prediction approaches have (as discussed in Section 2.3). The lack of known 3D protein structures for a large number of naturally occurring proteins ultimately restricts researchers to a small number of highly studied proteins [155]. In fact, less than $0.1 \%$ of the proteins for which the amino acid sequence is known has an accompanying experimentally determined 3D structure [187]. The Critical Assessment of methods of protein Structure Prediction (CASP) project aims to assess the state of the art in 3D protein structure prediction methods and has been in existence for over 20 years [188]. Recently, the progress of these methods was assessed by comparing the results over a ten year period. In spite of the fact that the number of experimentally determined 3D structures have increased dramatically over this time span, this did not help those methods which aim to establish a connection between a query protein sequence and predetermined 3D structures (template-based modelling). This is largely due to the fact that, although there is now more data, selecting the optimal template is now much more difficult. This problem lead to the overall accuracy of these methods staying unchanged over this time period [144]. One of the most interesting and difficult categories of structure prediction is free modelling where no connection can be made between the query protein and predetermined structures. Although a lot of progress has been made in this area, when 
assessing the results of the latest competition (CASP11), it was clear that many of the participating methods had a lot of difficulty with proteins of this type [145]. In these latest results a series of pitfalls were identified and it was clear that protein structure determination by computational methods remains an unsolved problem [137]. The fact that the end goal of many protein design methods is to create a protein with a predetermined 3D structure means that structure determination plays a major role in these approaches and, ultimately, limits their overall applicability.

What if, however, the ultimate goal of protein design (to create novel proteins with specific functional attributes) could be met without necessarily having to rely on structural information? Admittedly this fundamentally alters the stated problem many protein design approaches strive to solve, but a method able to do this would be able to avoid the draw backs associated with three-dimensional protein structure prediction. This would mean it could potentially be applied to a much wider array of problems and would not have to endure the computational intensity of protein structure prediction. This section will describe a novel approach named the In-Silico Protein Synthesizer (InSiPS) which aims to design proteins with specific PPI profiles. This approach solely relies on a set of known interacting proteins and their respective sequences with protein structure playing no role. To the author's knowledge, the design of proteins with specific PPI profiles which do not fundamentally rely on protein structure has not been done before and thus this represents a potentially uninvestigated approach to the design of functionally specific proteins.

InSiPS is a massively parallel computational tool designed to produce proteins with specific PPI-profiles. More specifically, given a target protein and a set of nontarget proteins, InSiPS aims to design a protein sequence such that it is predicted to interact with the target protein and predicted not to interact with the non-target proteins. This chapter will be organized as follows. In Section 7.2 the genetic algorithm, which InSiPS is built around, will be described. Following this, Section 7.3 describes the fitness functions used within the genetic algorithm and how and why it was decided upon. Section 7.4 describes the implementation of InSiPS and how it was integrated with MP-PIPE2. Section 7.5 describes performance and benchmarking tests carried out with InSiPS on two different compute clusters. InSiPS validation will be discussed in Section 7.6, including parameter tuning on real problems (section 
7.6.1) and wet-lab experimental validation (section 7.6.2). This work is also described in the following publication [252].

\subsection{The InSiPS Genetic Algorithm}

InSiPS is built around a Genetic Algorithm (GA), which is inspired by the evolutionary process of natural selection, to produce its novel protein sequences. A GA comes from the family of evolutionary algorithms, several of which have been utilized by recent structure based protein design algorithms [61]. The aim of a GA is to solve a given optimization problem and generally works as follows. First, potential solutions to the problem need to be encoded into a string of some sort to be easily manipulatable. An initial pool of potential solutions is then created, often times they are randomly generated. These solutions are then evaluated, with the better solutions more likely to be used in the future. Potential solutions are then chosen to be either randomly mutated or combined with other solutions to make a new generation of potential solutions. By doing this, better solutions are created which are then used to make further generations. This process is continued until some termination criteria is met, usually a solution with a given fitness has been reached or a certain number of generations have passed.

In this case, a problem takes the form of a target protein and a set of non-target proteins and a potential solution is simply an amino acid sequence making up a protein. The GA then aims to produce a protein sequence which optimizes the fitness function. For a given candidate protein sequence, this fitness function combines the PIPE scores that this sequence achieves against the target and non-target proteins as defined in the given problem. This fitness function is fully detailed in Section 7.3, however for now it is enough to know that this function assigns a given sequence a score between 0 and 1, with a higher fitness representing a better solution (ie. it is more likely to interact/not interact with the target/non-targets than sequences with a lower fitness score).

When InSiPS is presented with a problem, it begins by randomly generating a predetermined number of protein sequences. This set of sequences represents its initial population of candidate solutions. Any set of protein sequences can be used as a starting population, however to remove any forms of bias, a randomly generated 
set of sequences is usually used. Once an initial population is generated, the main loop of the algorithm is entered. This loop has the following steps:

\section{Evaluate Candidate Solutions.}

For each sequence in the current generation, PIPE is used to predict whether the sequence interacts with the target and non-target proteins. These scores are then combined using the fitness function described in Section 7.3. The fitness of a given sequence is a score between 0 and 1 and is used to chose which sequences will participate in the production of the next generation. The fitnesses of all of the sequences in the current generation are summed (total_fitness) and the probability that a given sequence $A$ is chosen in the next step is

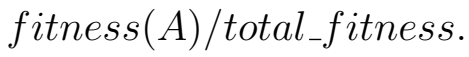

\section{Construction of the Next Generation.}

Once all of the sequences in the current generation have been evaluated and assigned a fitness score, they can be used to generate the sequences making up the next generation. As previously mentioned, when a sequence is chosen, it is done so at random with a probability proportional to its fitness with respect to the fitnesses of the other sequences in the current generation. To build the next generation, three operations are used, namely copy, mutate and crossover. These operations have a predetermined probability (p_copy, $p_{-}$mutate and $p_{\text {ccrossover }}$, respectively) at which they will be chosen to be used. These probabilities are set such that they always sum to 1 . These operations work in the following manner:

- Copy: When the copy operation is used, a sequence is simply chosen as described above to be directly copied into the next generation.

- Mutate: When the mutate operation is used, a sequence is chosen from the current population as described above. Then each amino acid in this sequence is mutated with a predetermined probability (p_mutate_aa). Typically this probability is relatively low to preserve most of the current state of the protein. When a spot mutation is performed, it is done purely at random, meaning a given amino acid is not more likely to mutate into one amino acid than another. 
- Crossover: When the crossover operation is used, two sequences $A$ and $B$ are chosen from the current pool as described above. A cut-point in the protein sequences is then randomly chosen, ensuring it is not too close to either end. The first portion of sequence $A$ is then joined with the second portion of sequence $B$ as well as the first portion of sequence $B$ being joined to the second portion of protein $A$ to create two new sequences to be added into the next generation.

These operations are repeated until enough sequences are generated and the next generation is complete.

This loop is then repeated until some termination criteria has been met. A variety of criteria can be used here, such as a maximum number of generations, target fitness or a maximum number of generations without any improvement. Once a chosen criteria is met, the best sequence generated is output to the user. This process is summarized in Figure 7.1.

\subsection{The InSiPS Fitness Function}

To be able to assess the quality of a given sequence produced by InSiPS, as well as being able to directly compare the quality of two generated sequences, a fitness function is needed. Typically, a GA's fitness function incorporates various attributes of the candidate solutions into a single score used to judge the quality of these solutions. In InSiPS's case, the attributes used are the PIPE predicted interaction scores for a given generated protein sequence against the target protein and the non-target proteins. The goal here is to combine all of these scores in some meaningful way. The chosen fitness function will need to balance the positive aspects of the sequence in question (its score against the target) as well as the negative aspects (its scores against the non-targets). To quantify how well a sequence scores against the nontargets, the PIPE scores are aggregated using a simple function such as average or maximum. Typically this will be problem specific. For example, for a given problem one may tolerate a few high scoring non-target proteins as long as most of the non-targets have a low score. This would lead to a situation where a small number of the non-targets may end up interacting with the generated sequence but the vast 


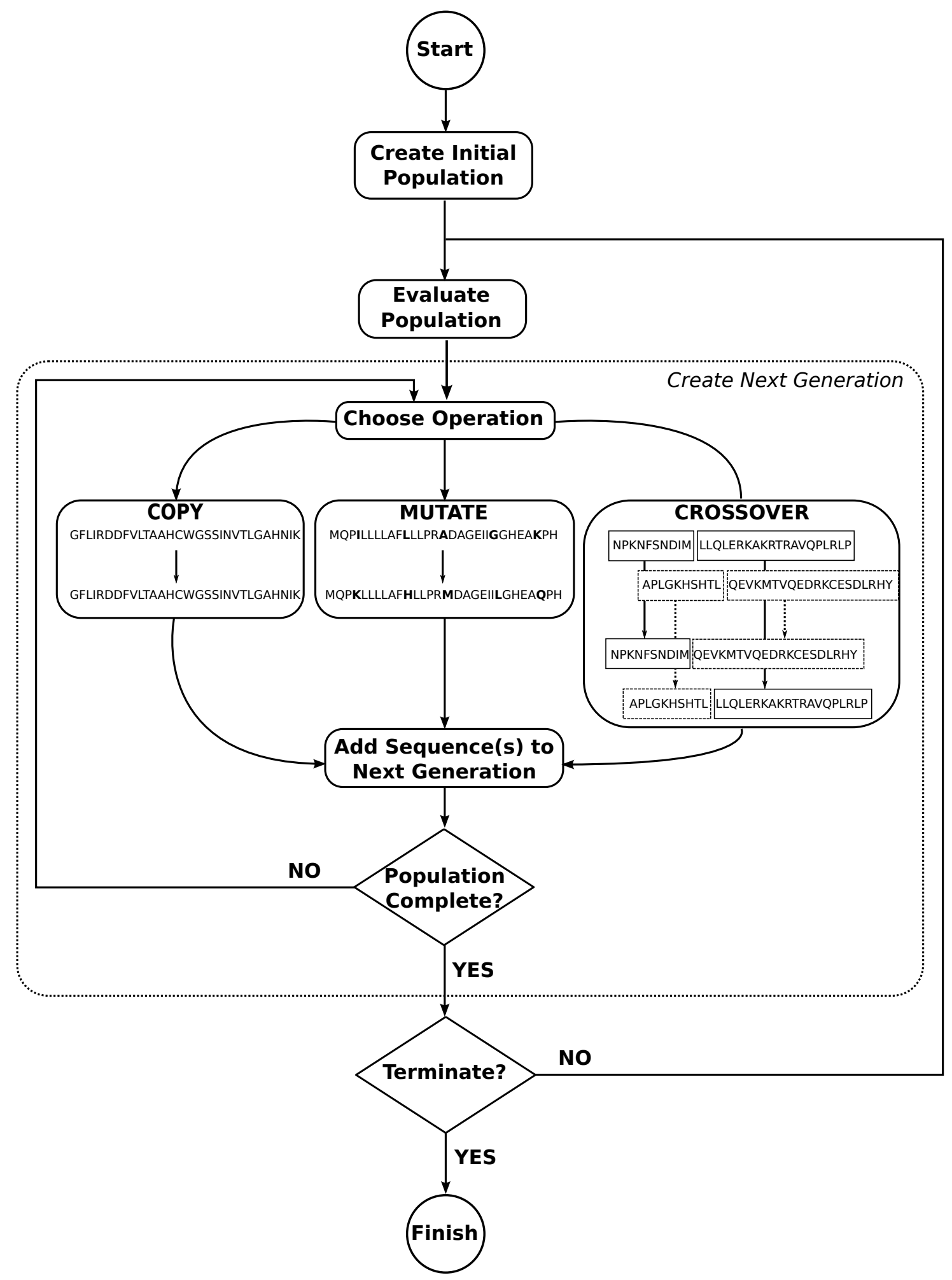

Figure 7.1: Graphical overview of the core InSiPS genetic algorithm. 
majority of them would not. In this situation one would use the average aggregation function. On the other hand, one may wish to avoid any non-target interactions at all costs while accepting overall higher non-target scores in general. In this case one would use the maximum aggregation function to summarize the non-target scores. Moving forward, this aggregation function will simply be referred to as the function $f$. Given this, the fitness function will now incorporate both target score (henceforth denoted as PIPE(sequence, target)) and the aggregate non-target score (henceforth denoted as $f(\mathrm{PIPE}($ sequence, non-targets $)))$. For a given problem, an ideal solution would be a protein sequence with a very high PIPE(sequence, target) score and very low $f(\mathrm{PIPE}$ (sequence, non-targets)) score where as a poor solution would have a low PIPE(sequence, target) score and a high $f(\mathrm{PIPE}$ (sequence, non-targets)) score.

With the above in mind, the goal of the fitness function was to apply a score between 0 and 1 for a given sequence based solely on the PIPE(sequence, target) and $f$ (PIPE(sequence, non-targets)) scores (both also between 0 and 1 ). If one were to plot the ideal fitness function as a heat map, with PIPE(sequence, target) score on the $x$-axis and $f(\mathrm{PIPE}$ (sequence, non-targets)) score on the $y$-axis, it should be very low scoring in the top left corner (low PIPE(sequence, target) score and high $f(\mathrm{PIPE}$ (sequence, non-targets)) score), very high scoring in the bottom right corner (high PIPE(sequence, target) score and low $f(\mathrm{PIPE}$ (sequence, non-targets)) score) with a gradient of scores in between. The fitness function for a given sequence (seq) generated by InSiPS is given below.

$$
\text { fitness }(\text { seq })=(1-f(\operatorname{PIPE}(\text { seq, non-targets }))) * \operatorname{PIPE}(\text { seq, target })
$$

This fitness function is also plotted as a heat map in Figure 7.2. This function achieves exactly what was intended. As the PIPE(sequence, target) score rises or the $f$ (PIPE(sequence, non-targets)) score lowers, the fitness rises. If these things happen simultaneously the fitness rises even faster, which is exactly what one would hope for.

Although this fitness function combines disparate aspects of the generated proteins (how well it interacts with the the target protein, how well it avoids interaction with the non-target proteins), it does not necessarily need to be implemented in this 


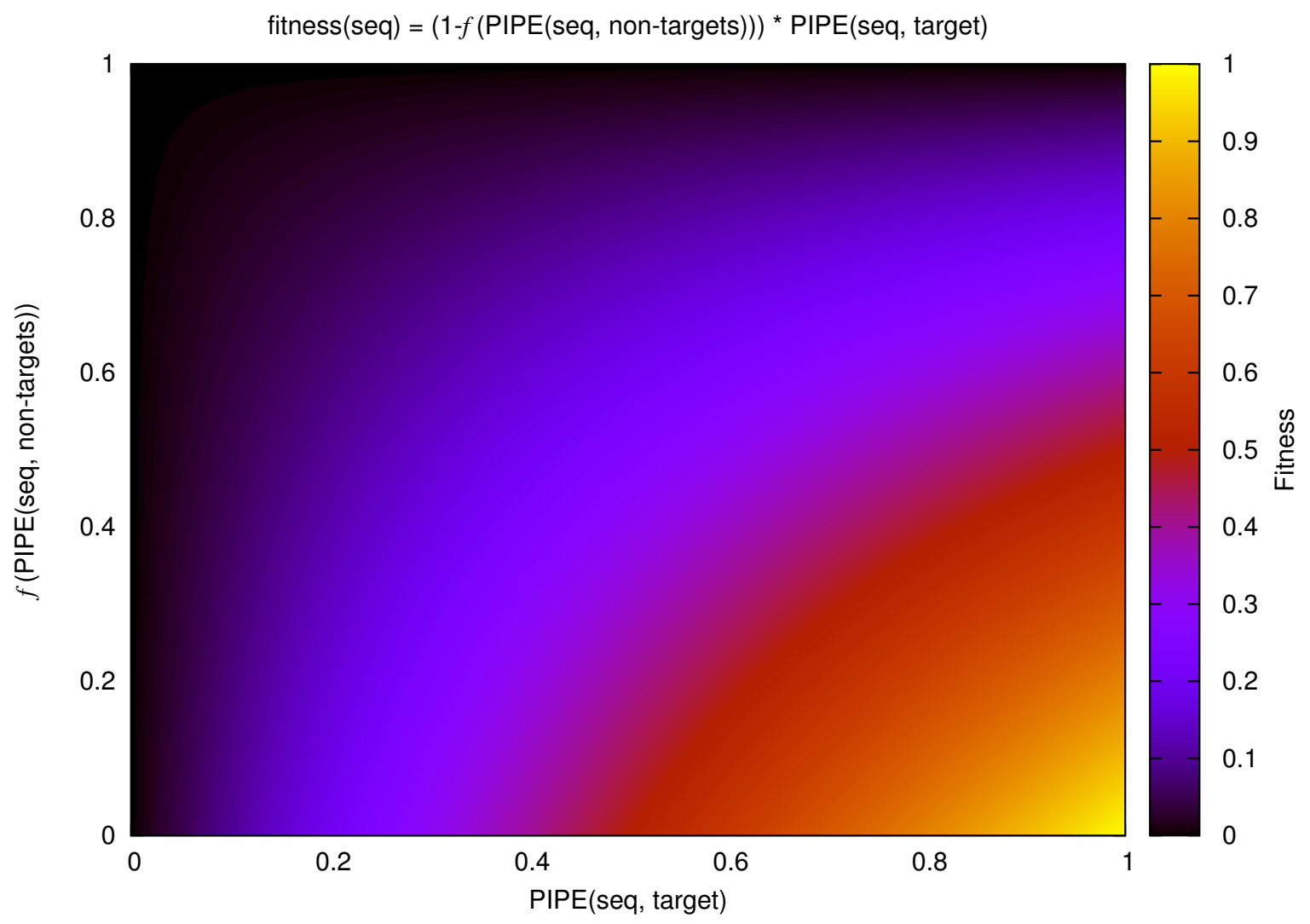

Figure 7.2: Heat map of the InSiPS fitness function.

manner. This optimization problem could be recast as a multi-objective optimization problem, where these aspects (and possibly others, including predicted physical or chemical properties of the generated proteins) are not combined into one fitness score, but are all maintained separately and the algorithm would try to optimize all of them in some manner. The idea of multiple criteria decision making (which includes multiobjective optimization) has been an area of study for decades [116]. These ideas will not be explored here but offer an avenue of potential future research.

\subsection{The InSiPS Implementation}

In this section a general overview of the InSiPS implementation is given. It is meant to give the reader an insight of InSiPS's development route, improvements over previous PIPE implementations, interesting or necessary implementation decisions made 
followed by a full overview of how the master and slave processes which make up InSiPS work. The current implementation is a heavily modified version of MP-PIPE2, consisting of a very similar, two-level master-slave/all-slaves parallel architecture. For more information on the MP-PIPE2 implementation see Section 3.3 or [250, 253]. It should be noted that the current version of InSiPS has been developed and tested using $S$. cerevisiae data.

Initially InSiPS was implemented as a hybrid program. The GA portion of the algorithm was implemented in Python and was responsible for creating, evaluating and manipulating the protein sequences to produce generation after generation, slowly finding improved solutions to the given problem. As a part of the evaluation, this program would need to call the MP-PIPE2 implementation. However, it would first need to create the PIPE database files for the protein sequences created in the current generation (as described in Section 4.2.2). Once this was done, the Python program would create the relevant input files and then MP-PIPE2 would be run. For each sequence in the current population, MP-PIPE2 would be run on it with the target protein as well as with all of the non-targets. MP-PIPE2 would simply load the newly created database file for the sequence in question as well as the target/nontarget file and make an interaction prediction as it normally would. It should be noted that the traditional PIPE score (as opposed to the modified, weighted PIPE scoring function described in Section 4.2.2) is used by InSiPS. The reason for this is that when using the traditional scoring function, introducing a new protein sequence with no known interactions (which InSiPS does every generation) to MP-PIPE2 only requires one to generate a database file for this new sequence. This is because, for the traditional score function, only proteins with known interactions are relevant within the database files. However, the modified score function weights the traditional score by how common certain amino acid motif pairs are within the pairs of interacting proteins (ie. if a pair of motifs are very common they are unlikely to support a possible interaction, see Section 4.2.2 for more details). Because of this, if one wanted to use the weighted function with InSiPS, the entire collection of database files would need to be regenerated each time a new set of sequences were introduced (ie. each generation). It was decided that this computational expense was not worth the slight boost in prediction accuracy and that the traditional PIPE score would be used for 
InSiPS.

Although this implementation worked, it was very inefficient and was used merely as a proof-of-concept. The major inefficiency is the repeated calling of the MP-PIPE2 program. When doing this, MP-PIPE2 would need to reload all of the relevant data from disk before being able to make any predictions. On top of this, the GA would need to write a significant amount of data to disk to be used as input to MPPIPE2 and then would need to read in the output to then calculate the fitness for each sequence. For these reasons, it was decided to fully merge the GA into the C implementation of MP-PIPE2. The GA would simply become part of the master process which would then distribute sequences (instead of protein pairs) to the slave processes. These slave processes would then predict whether the sequence interacts with the target and non-targets.

Before describing how the entire current InSiPS system works, one major improvement to the MP-PIPE2 implementation needs to be discussed. As described above, when presented with two proteins $A$ and $B$, MP-PIPE2 would simply load the two database files for these proteins from disk and then proceed with making its prediction. When presented with a second pair of proteins $C$ and $D$, the previously loaded files are discarded and the currently relevant files are loaded. As described earlier, MP-PIPE2 is capable of making a large majority of its predictions in a fraction of a second. When performing a large run with a number of slave processes each using a large number of threads, this would lead to the hard disks being inundated with read requests. This problem only worsens when using a modern compute cluster whose disk storage is comparatively very slow. To solve this problem, a new approach to how this data is stored and accessed by the slave processes was designed. As previously mentioned, traditionally each protein's data was stored in a single database file which was loaded when needed. This data is stored in binary files on disk as they are simply a collection of short integers. To be able to access this data more efficiently, it was decided to store all of the database as one contiguous file. This meant that one large binary database file would replace the thousands of individual database files. The data in this file would be loaded into a large short integer array and stored in memory. This array comprised the original database files concatenated together. The only issue with this is that the database files are of varying size (due to some proteins 
being more similar to the other proteins than others). Instead of creating wasted buffer space to allow each entry in the unified database the same amount of memory, an index was created. Internal to all of the PIPE implementations, the proteins in question are referred to by a unique integer ID. When creating the database, the start location of each protein's data in the database is recorded. This data is then stored in the database file. Once it it read along with the entire database itself, it is used as follows. When presented with two protein $A$ and $B$, the system simply looks up where the data pertaining to these proteins are in the database in the index. This provides two pointers into the database. At the end of the pointer is information as to how far from that point to read to retrieve all of the protein in question's data. So the system simply retrieves protein $A$ and $B$ 's data from the database and proceeds to make a prediction. When it is then presented with proteins $C$ and $D$, the index and database are referred to in exactly the same manner. This completely removes the need for the slave processes to access the hard disk at all, leading to a potentially large amount of saved time (depending on the computing resources used).

To fully utilize all available computing resources and to minimize idle time, it was decided to fully integrate the InSiPS GA into the MP-PIPE2 implementation. The GA was embedded into the MP-PIPE2 master process and the MP-PIPE2 slave processes work in largely the same manner. As in the MP-PIPE2 implementation, each processes uses a user-defined number of computational threads. Ideally one would assign an InSiPS process to each available shared memory compute node. The user would then set the number of compute threads appropriately to fully utilize each node. For example, if one had access to a cluster of 8 quad-core desktop PCs, the ideal setup would be to run 8 InSiPS processes (1 master and 7 slaves), each using 4 threads. The pseudocode for the InSiPS master process is given in Algorithm 1.

When the program starts, the first change from the MP-PIPE2 implementation is seen. In the MP-PIPE2 implementation, each process would load the necessary data from disk itself. Generally speaking, all of this data is centrally located, which means this was an unnecessary bottleneck. This bottleneck would only worsen with slow hard disks or a large number of slave processes. The first thing the InSiPS master process does is to load all relevant data (known PPI graph, PIPE similarity database and index, real protein sequences and the current target and non-targets) and then 
Algorithm 1: InSiPS master process.

load known protein-protein interaction graph from disk

load PIPE similarity database \& index from disk

load real protein sequences from disk

load targets \& non-targets from disk

broadcast all loaded data to slave processes

current_generation $\leftarrow \emptyset$

foreach computational thread in parallel do

while current_population is not full do

L add random protein sequence to current_generation

while termination criteria is not met do

while unanalyzed sequences remain in current_generation do

receive work request from slave $x$

receive previous results from slave $x$

send sequence from current_generation to slave $x$ for analysis

foreach computational thread in parallel do

while members of current_generation don't have assigned fitness do choose a sequence without an assigned fitness

- use results obtained from slave processes to assign fitness score

next_generation $\leftarrow \emptyset$

foreach computational thread in parallel do

while next_generation is not full do

randomly choose a GA operation

select sequence(s) from current_generation

apply GA operation \& add new sequence(s) to next_generation

current_generation $\leftarrow$ next_generation

foreach slave process do

receive work request from slave $x$

send END signal to slave $x$ 
broadcasts this data to all of the slave processes. Once this is done, the initial population is created, in parallel, by each thread simply creating random protein sequences and adding them to the population until it is full. The main GA loop is then entered. Once inside this loop, the first thing done is the evaluation of the current population. The master process waits for work requests from the slave processes and, when one is received, the master process then receives any previous work that slave process has done before sending it a sequence from the current population. It is then the slave process' responsibility to produce PIPE prediction scores for this sequence against the target and non-targets. Once all of the sequences have been processed in this manner, their fitness is then calculated in parallel. Each computational thread takes a sequence and, using the PIPE prediction scores generated by the slave processes, it calculates that sequence's fitness (as described in Section 7.3). Once all of the sequences have been assigned a fitness, the next generation can be created. In parallel, each thread randomly decides which GA operation it will perform based on the user defined probabilities these operations have been given. Once one of these operations (copy, mutate or crossover) have been chosen, the thread then randomly selects one or two proteins (depending on which operation was chosen) from the current generation. The probability that a given sequence is chosen is based on its fitness relative to the rest of the population. The computational thread then applies the chosen operation to the selected sequence(s) and adds the new sequence to the next generation. Once the next generation is full, it is set as the current generation and the main GA loop starts again until some termination criteria is met. Once the termination criteria is met, the master process informs the slave processes and the program exits.

The pseudocode for the InSiPS slave process is given in Algorithm 2. As previously mentioned, the slave processes no longer load any data from disk and has it all sent over the network from the master process, relieving considerable stress form the underlying hard disks. The InSiPS slave processes are solely responsible for making PIPE predictions with the generated sequences and the current target and non-targets. They first send a work request to the master process. If they do not receive the signal to stop, then then send any previous results it may have generated. It then receives a generated sequence from the master process. To be able to run PIPE on this sequence and the target/non-targets it needs the respective PIPE similarity 


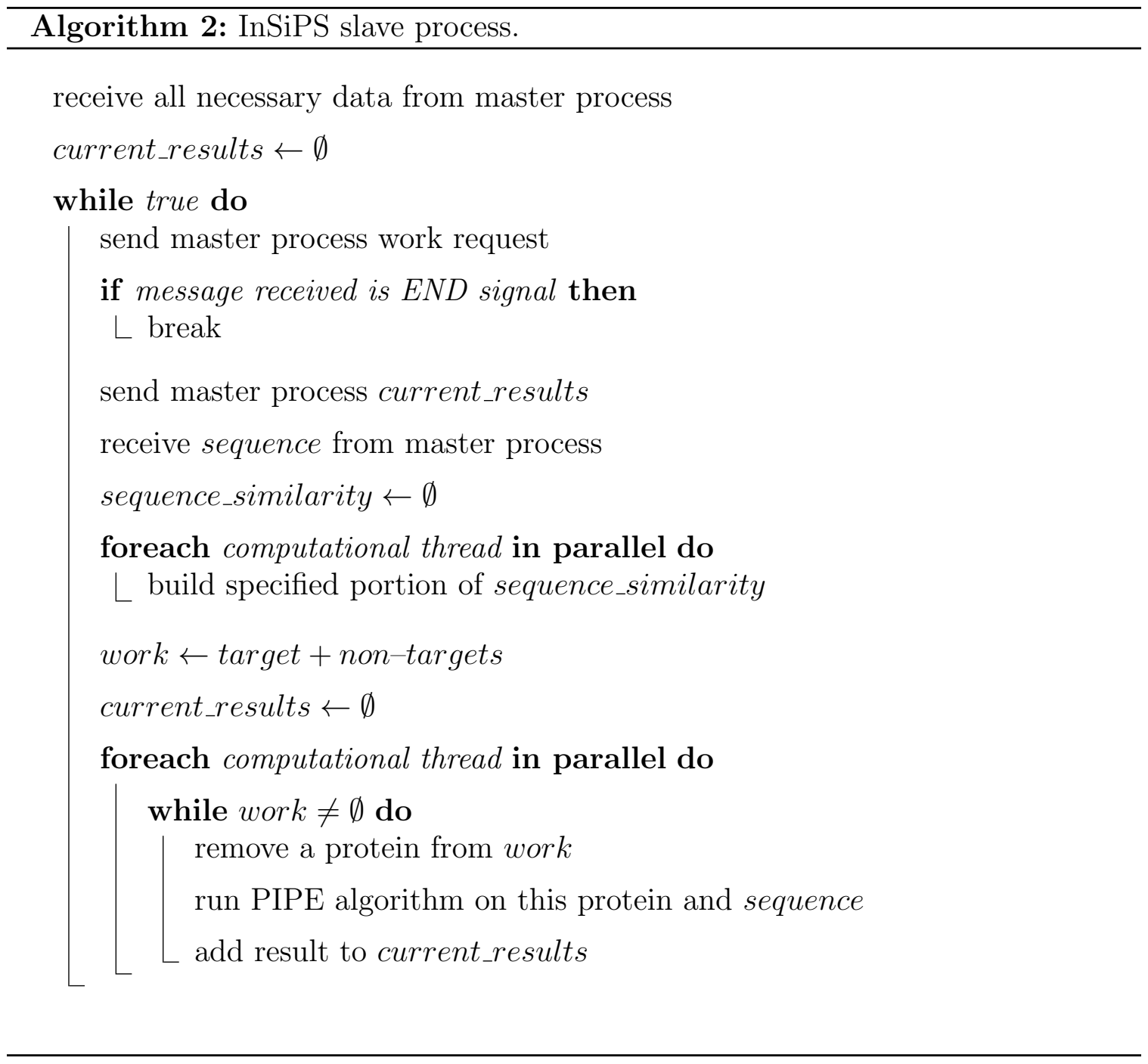

database file (as described in Section 4.2.2) to be generated. The relevant files for the target and non-targets are found within the database sent from the master process. These files do not need to be updated if, when running the actual PIPE algorithm, the new sequence is the first protein investigated. To accomplish this, the database file is created in parallel with the use of the real protein sequences sent from the master process and stored in memory for use by all of the computational threads later. Once this is completed, while there are still proteins within the target and non-targets that have not been processed, each computational thread takes one of these proteins and runs the PIPE algorithm with this chosen protein and the current sequence with the use of the newly generated database file and the standard PIPE database sent by the master. Both of these data structures are read-only and therefore can be accessed 
by all of the threads simultaneously. Once all of the proteins have been analyzed, the slave process notifies the master process by sending a work request. This loop continues until the master process notifies the slave processes that there is no more work to be done.

\subsection{InSiPS Performance Testing and Benchmarking}

To benchmark InSiPS, it is necessary to see how well the code scales as more threads are used per process, as well as the impact of using more slave processes, has on the overall run time. These benchmarks were broken down into two separate tests and both of these tests were run on the Carleton Parallel Computing \& Data Science Research Lab cluster (consisting of a number of quad-core desktop PCs) as well as Scinet's BlueGene/Q (BGQ) cluster. First how the code scales as more threads were used by the slave processes will be evaluated followed by an evaluation of how the number of slave processes used influences the run time.

\subsubsection{Benchmarking Test 1: Number of Compute Threads Used}

The focus here was put on the slave processes since nearly all of the computation time is spent performing PPI predictions. This test measures the entire time it takes the slave process to receive the sequence from the master, build the necessary similarity data structure and carry out PPI predictions between this sequence and all 6,707 yeast proteins. In this test, five different protein sequences with a range of computational difficulty were evaluated. These sequences (listed easiest to hardest) are: YPL108W, YPL158C, YJR151C, YCL019W and YHR214C-B. As can be seen in the run time results (Figure 7.3) and the speedup results (Figure 7.4) on the lab cluster, a dramatic decrease in run time using up to 4 threads (nearly linear speedup) followed by a further reduction of run time using up to 8 threads, at which point the performance plateaus, can be seen. On the BGQ cluster a similar trend is observed (Figures 7.5 and 7.6). Perfectly linear speedup when using 16 threads and close to linear speedup when using up to 32 threads is observed. At this point an additional improvement in performance can be seen when using up to 64 threads, which happens to be the imposed thread limit on the BGQ cluster nodes.

The results for this test are very similar on both clusters used. In both cases an 


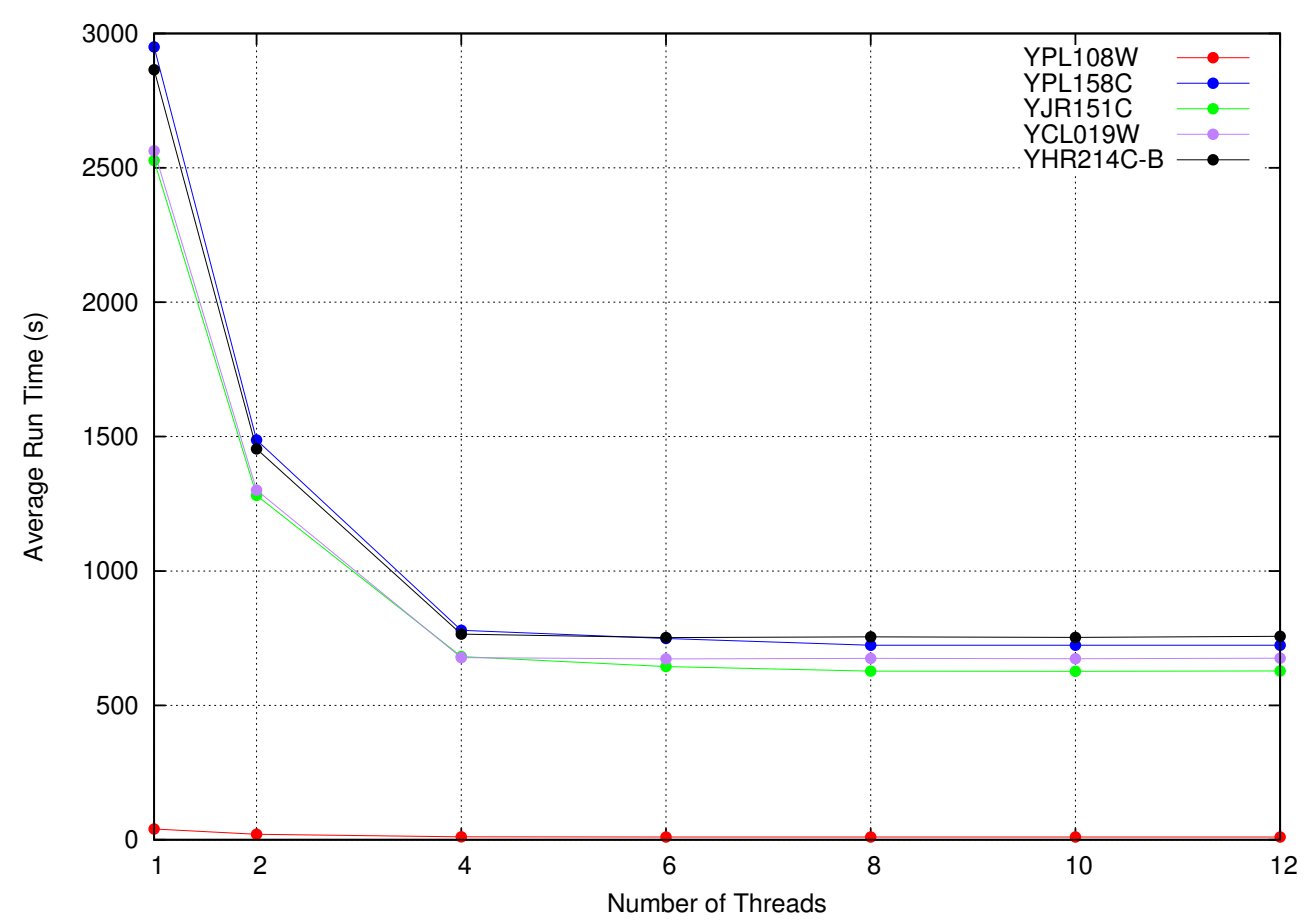

Figure 7.3: Run time results for InSiPS threads benchmarking test on the local lab cluster.

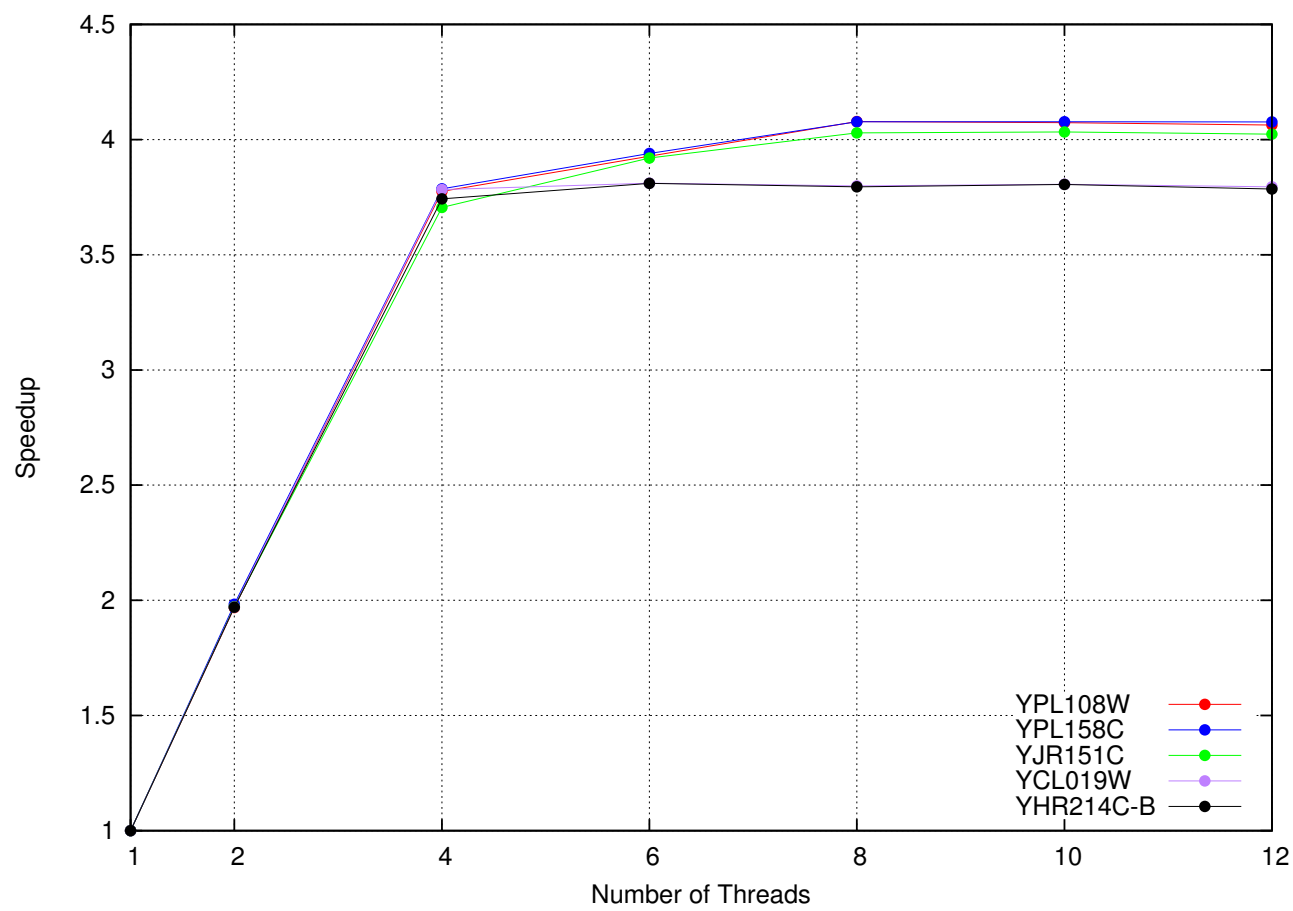

Figure 7.4: Speedup results for InSiPS threads benchmarking test on the local lab cluster. 


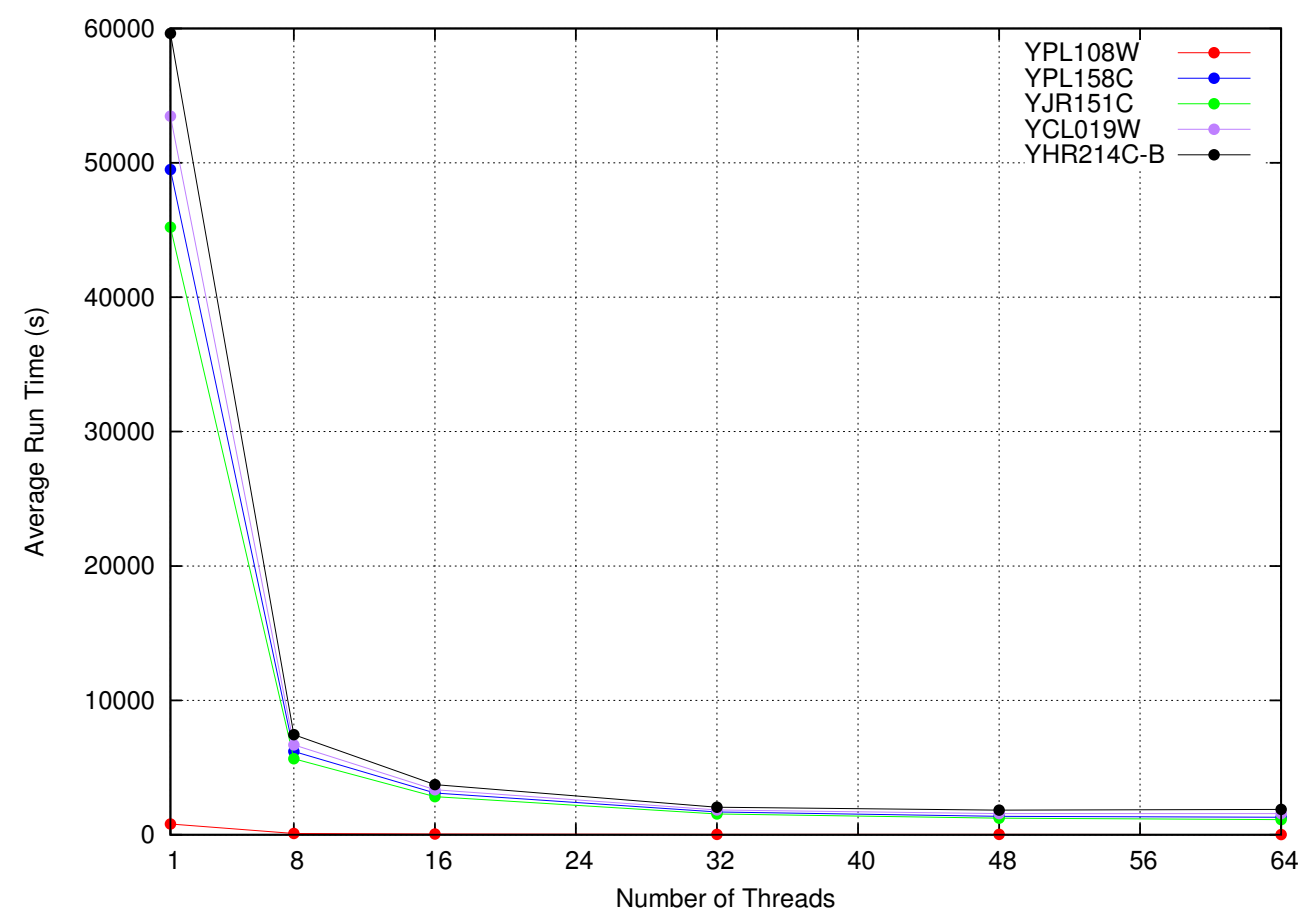

Figure 7.5: Runtime results for InSiPS threads benchmarking test on the Scinet BGQ cluster.

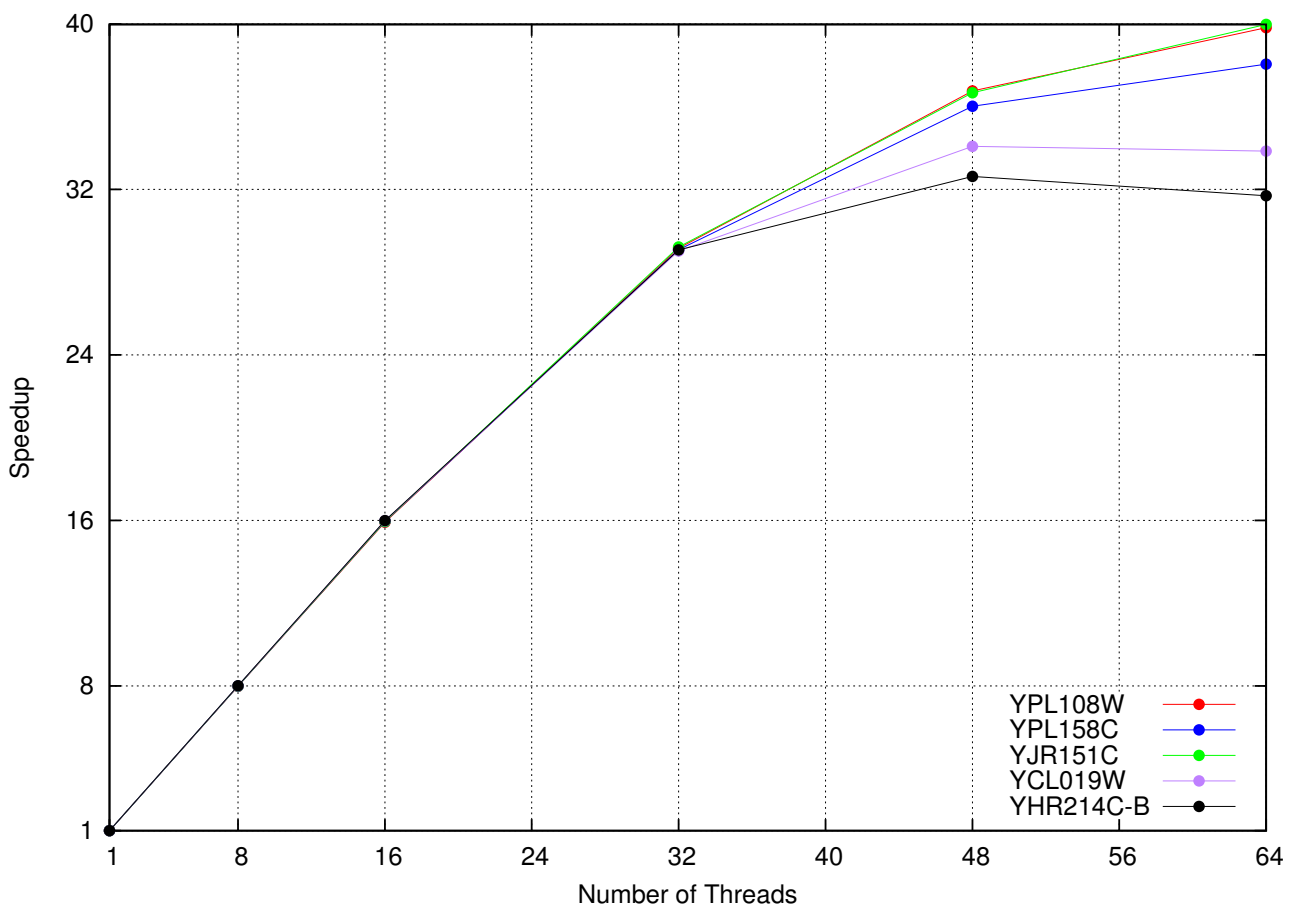

Figure 7.6: Speedup results for InSiPS threads benchmarking test on the Scinet BGQ cluster. 
extremely good performance improvement (close to linear speedup) when using the same number of threads as physical cores available (4 on the lab cluster and 16 on the BGQ cluster) can be seen. From here an improvement in performance (although not as dramatic) is still seen when using up to the number of hardware supported threads on each architecture ( 8 on the lab cluster and 64 on the BGQ cluster). The reason why a reduction in performance improvement is hit when using more threads than the number of physical cores available is due to the fact that InSiPS is memory I/O bound. Since the algorithm does not contain any floating-point arithmetic, the threads spend most of their time doing memory look-ups. When each thread is assigned its own physical compute core with its own access to main memory then they will not interfere with each other and good performance will be seen. However, when the physical cores are overloaded with computational threads and need to share the communication channels with main memory, a reduction in overall speedup occurs. If InSiPS had a significant amount of number crunching to do then each thread would not need to visit memory as often as it does, which means it could simply work on the local data is has. This would mean that the hardware supported threads would perform better as they each have their own set of registers which is where the local data is stored. As can be seen in the lab cluster results, the performance plateaus once all hardware supported threads are used. This indicates that overloading the systems do not provide any additional performance benefits. Tests of this nature could not be carried out on the BGQ cluster as there is an imposed hard limit of 64 threads per node.

\subsubsection{Benchmarking Test 2: Number of Slave Processes Used}

The second benchmarking test was done to see how well the code scales when more cluster nodes (ie. more slave processes) are used. These tests measured the entire time it took for a generation to be computed. This consists of the master process disseminating the sequences to the slave processes, the slave processes to first generate their similarity data structure and then run the PIPE PPI prediction method against the targets and non-targets, the slave processes sending the results back to the master

process, the master process then calculating the fitness of each sequence and finally the master processes generating the sequences for the next generation. The test 
problem in question was generated by choosing 260 random targets and non targets and sequence population consisted of 1500 sequences. It should be noted that the time it takes for a given sequence to be processed is largely based on its complexity (how similar it is to real proteins, how many proteins it is predicted to interact with, ect). For this reason, this test was performed on three different sequence populations. The first population was a randomly generated set of sequences which represents a typical start point for InSiPS. The second and third populations were the populations taken after running InSiPS after 100 and 250 generations, respectively.

Firstly, these tests was performed on the lab cluster. In these tests each process (master/worker) was placed on its own compute node. The tests started with one slave process and increased this to show how the code scales when more resources are used. Secondly, these tests were performed on the BGQ cluster. Here the minimum number of nodes to use for a job is 64 , so this was used as a baseline (1 master process, 63 slave processes). Multiples of 64 nodes were then added to see how well the code scales.

As can be seen in the results of this test on the lab cluster (Figures 7.7 and 7.8) the code scales extremely well on this cluster (perfectly linear). The results on the BGQ cluster (Figures 7.9 and 7.10) are also encouraging while not as great as on the lab cluster. When InSiPS uses an extreme number of nodes (1024), a slight drop-off from linear speedup (12x as opposed to $16 \mathrm{x}$, which would be perfect) is observed. This most likely results from two things. First, it is possible that when there are this many worker processes, the master process becomes slightly overwhelmed by work requests and some slave processes might have to wait for their requests to be fulfilled. This results in the slave process idling for a short period of time thus contributing to the lack of perfect scaling. Secondly, a portion of the computation is done only by the master process every generation (the fitness calculation as well as generating the sequences for the next generation). This part of the computation can not benefit from the addition of more cluster nodes, although it is computed in parallel. Luckily this portion only represents a small percentage of the overall computation and therefore does not have a massive impact. These things considered, InSiPS performs very well even on an extremely large number of nodes. 


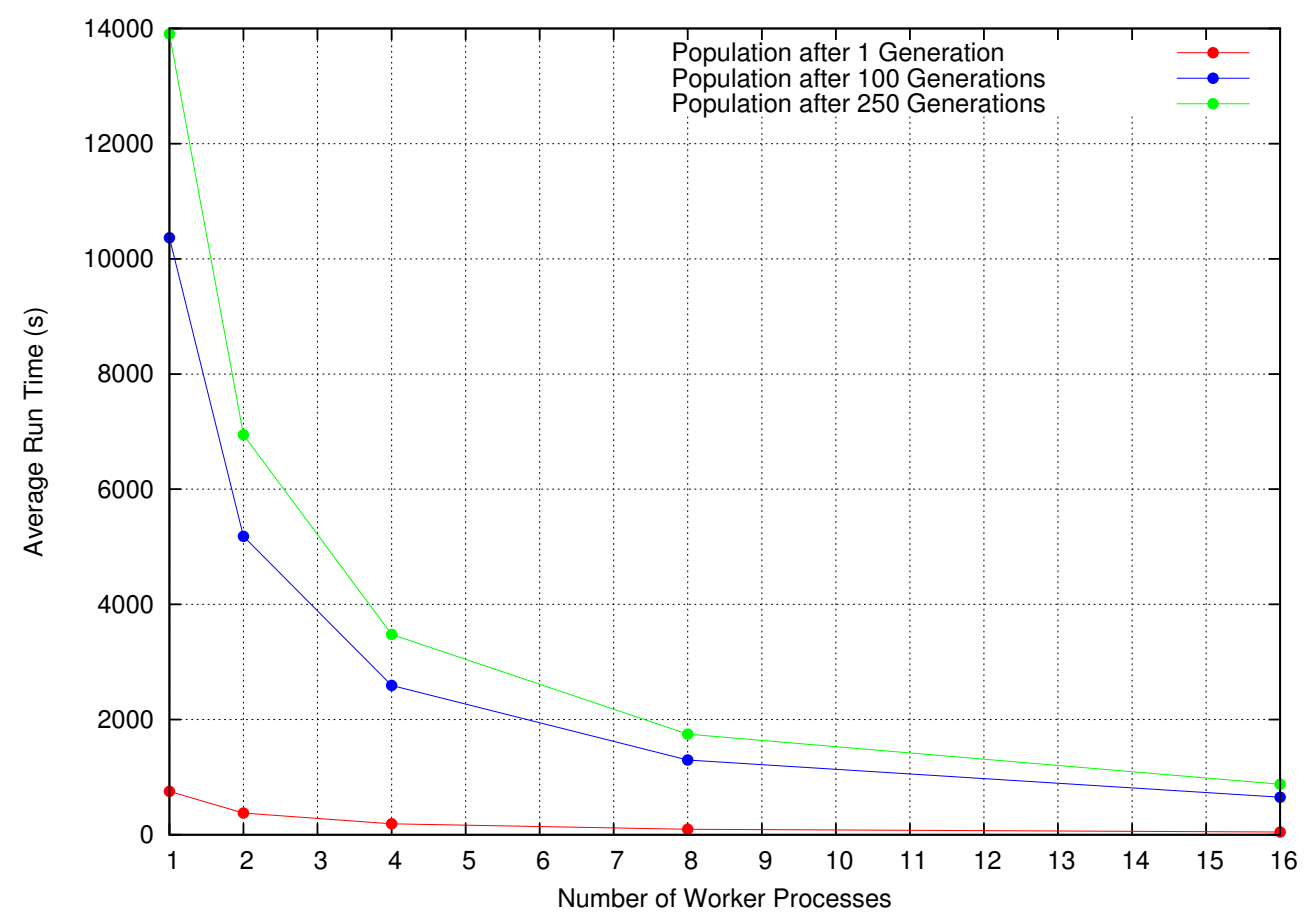

Figure 7.7: Run time results for InSiPS worker process benchmarking test on the local lab cluster.

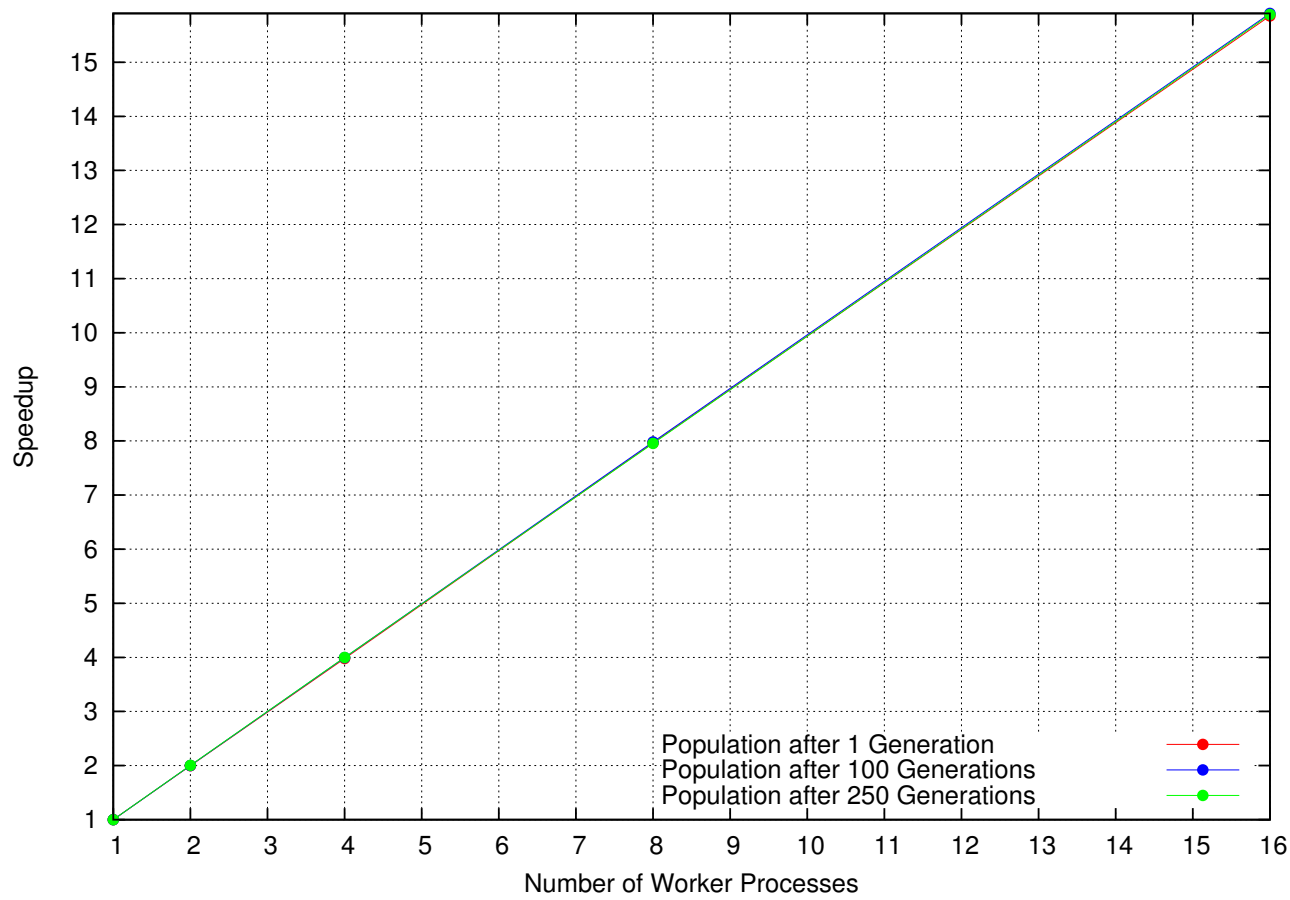

Figure 7.8: Speedup results for InSiPS worker process benchmarking test on the local lab cluster. 


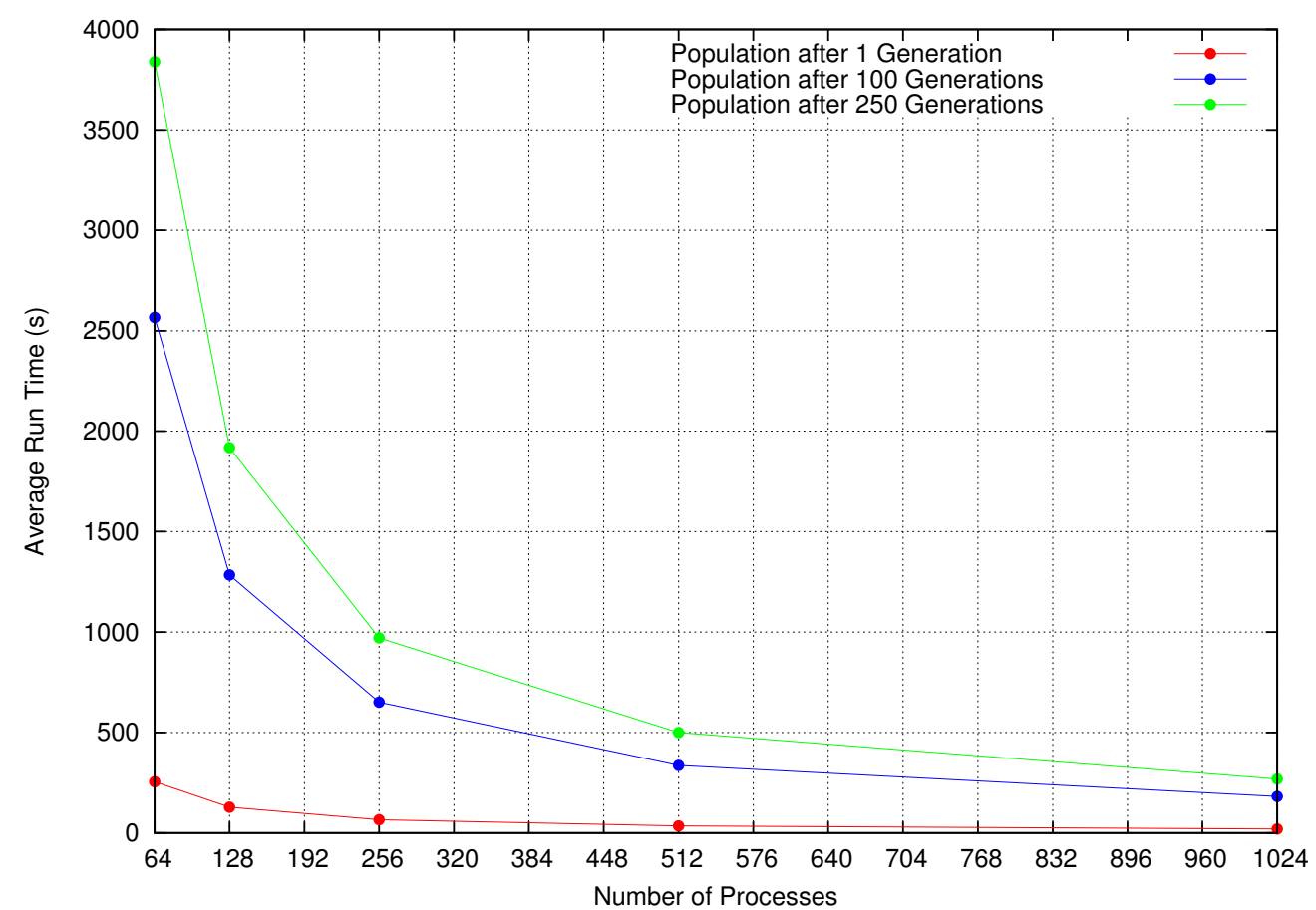

Figure 7.9: Runtime results for InSiPS worker process benchmarking test on the Scinet BGQ cluster.

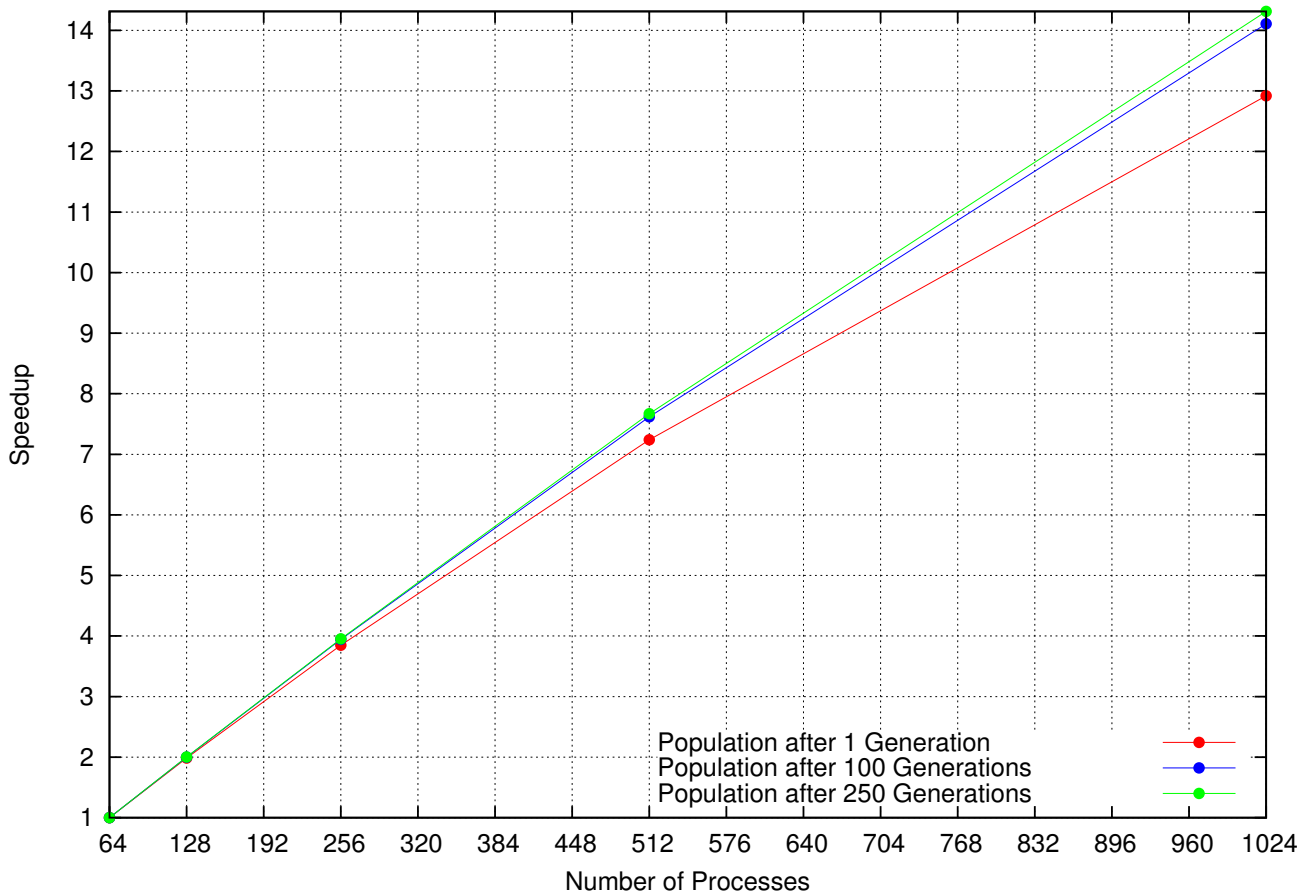

Figure 7.10: Speedup results for InSiPS worker process benchmarking test on the Scinet BGQ cluster. 


\subsection{Experimental Validation on Real Problems}

To evaluate how well InSiPS performs on real, testable scenarios, a set of candidate problems was created. To make these problems amenable to wet-lab experimentation, this set of problems had targets with the following properties:

1. Cytoplasmic (proteins only ever shown to localize in the cytoplasm)

2. Relatively small in size (less than 1,500 amino acids in length)

3. Moderate level of abundance

4. Absence of the protein resulted in increased sensitivity of the cell to a well defined external stimuli/stressor

The task was to create a protein which would bind to one of these cytoplasmic proteins of interest (target) and not to any of the other 1,701 cytoplasmic proteins (non-targets). 18 of these candidate target proteins were identified and are listed in table 7.1.

\subsubsection{InSiPS Parameter Tuning}

As previously mentioned, the probability that a given operation is used during the creation of a new sequence by InSiPS's GA is predefined by the user. These key parameters, namely p_copy, p_mutate and p_crossover, will shape the way InSiPS builds new sequences in search of a sequence which satisfies the termination criteria for a given problem. The only restriction on these parameters is that they must sum to 1.0, otherwise any combination of real number values will work. Given that these are real number parameters, the parameter space is absolutely massive in practice (and, in theory, infinite). It is tempting to try to find the best set of parameters, however in this case the time needed to do so (even using smart heuristic searches) would be prohibitive. The reason for this is that it is not only these parameters that have an effect on how the program operates, but the random numbers generated also play a large role. It is very possible, and indeed likely, for two separate runs of InSiPS on the same problem with the same parameter settings to produce solutions of vastly different quality due to the stochastic nature of InSiPS's GA. For example, if in one 


\begin{tabular}{cccc} 
Gene Name & Common Name & Protein Length & Abundance (Molecules/Cell) \\
& & & \\
RMD1 & YDL001W & 430 & 952 \\
FUN11 & YAL036C & 369 & 11,700 \\
CDC24 & YAL041W & 854 & 1,010 \\
AIM2 & YAL049C & 246 & 1,800 \\
ACS1 & YAL054C & 713 & 2,890 \\
BDH1 & YAL060W & 382 & 8,730 \\
CCR4 & YAL021C & 837 & 2,780 \\
HEK2 & YBL032W & 381 & 15,600 \\
PIN4 & YBL051C & 668 & 4,630 \\
YPT10 & YBR264C & 199 & 1,360 \\
REI1 & YBR267W & 393 & 830 \\
EFM2 & YBR271W & 419 & 217 \\
HSM3 & YBR272C & 480 & 468 \\
CHK1 & YBR274W & 527 & 2,530 \\
PRD1 & YCL057W & 712 & 8,910 \\
PSK1 & YAL017W & 1,356 & 967 \\
PSK2 & YOL054W & 1,101 & 2200 \\
RPL19B & YBL027W & 189 & 225,000 \\
& & & \\
\hline
\end{tabular}

Table 7.1: Set of $S$. cerevisiae proteins making up the candidate targets for InSiPS.

run the randomly generated starting pool contained a few very good sequences, this could act as a head start over a second run, one of which the second run may only recover from after a number of generations. Another thing to keep in mind is that it is very probable that a given set of parameters works better for one problem than it does another. As previously stated, to do a real, in-depth study of these parameters (keeping in mind the effects that the random seed used or test problem in question can have on InSiPS's performance) is time prohibitive. However, it is clearly important enough to at least try a handful of different settings to see how these three aspects interact with respect to the final sequence quality.

To investigate this, three of the experimental candidate problems were chosen. The targets here were proteins YAL054C, YBR274W and YOL054W. To test how using different parameter sets affected the overall performance, five different parameter settings were used. In these settings, p_copy was kept consistent at 0.10 (since this 
operation doesn't actually add anything new to the next population). Also, in each of these settings, when the mutate operation was chosen each amino acid in the selected protein sequence would be randomly changed to another amino acid with a probability of 0.05 . This seemed a reasonable mutation rate as it would largely maintain the state of a given sequence while still introducing a degree of random change. The settings of the other parameters were:

- Parameter set 1: p_crossover $=0.45$, p_mutation $=0.45$

- Parameter set 2: p_crossover $=0.30$, p_mutation $=0.60$

- Parameter set 3: p_crossover $=0.60$, p_mutation $=0.30$

- Parameter set 4: p_crossover $=0.75$, p_mutation $=0.15$

- Parameter set 5: p_crossover $=0.15$, p_mutation $=0.75$

These parameter settings give a good sampling of different scenarios one might want to use from the balanced approach (parameter set 1) to a set heavily biased in favour of one operation (parameter set 4 or 5). To try to account for the shear amount of randomness involved in the running of InSiPS, each problem was run with each parameter setting using three different random seeds. When a random number generator is seeded with a given number, it will always produce the same series of random numbers. This way it can be assured, for instance, that two different runs of InSiPS has the same initial population. Typically InSiPS is not seeded with a specific seed as different sets of random numbers are generally desirable. That said, there are three problems, each using five different parameter sets, each being run with three different random seeds for a total of 45 runs. To add to this, two different variations on the fitness function described in Section 7.3 will be used. As previously explained, the fitness function needs to use some kind of aggregation function to summarize the PIPE prediction scores of the non-targets for a given sequence. In these tests both the maximum and average aggregation functions will be tested, as these could be expected to be used in real situations. The goal here is to see if one parameter setting produces better sequences consistently across different problems and different random seeds. InSiPS was run for 50 generations in each of these tests. The results for these tests can be seen in Tables 7.2 and 7.3 for the average and maximum aggregation functions, respectively. 
Target Parameter Setting $\quad$ Seed $1 \quad$ Seed $2 \quad$ Seed 3 Avg. Fitness

YAL054C

$\begin{array}{lcccc}1 & 0.589051 & 0.412247 & 0.590377 & 0.530558 \\ 2 & 0.573568 & 0.570626 & 0.53582 & \mathbf{0 . 5 6 0 0 0 5} \\ 3 & 0.540558 & 0.583261 & 0.50301 & 0.542276 \\ 4 & 0.508542 & 0.481411 & 0.528919 & 0.506291 \\ 5 & 0.475457 & 0.499648 & 0.453816 & 0.476307\end{array}$

Avg. Fitness

$\mathbf{0 . 5 3 7 4 3 5} \quad 0.509439 \quad 0.522388$

YBR274W

$\begin{array}{lcccc}1 & 0.659956 & 0.659754 & 0.633791 & 0.651167 \\ 2 & 0.697195 & 0.602428 & 0.636653 & 0.645425 \\ 3 & 0.711140 & 0.698402 & 0.650747 & \mathbf{0 . 6 8 6 7 6 3} \\ 4 & 0.640189 & 0.622758 & 0.617484 & 0.626810 \\ 5 & 0.550892 & 0.533373 & 0.550839 & 0.545035\end{array}$

Avg. Fitness

$\mathbf{0 . 6 5 1 8 7 4} \quad 0.623343 \quad 0.617903$

YOL054W

Avg. Fitness

$\begin{array}{ccccc}1 & 0.621445 & 0.679293 & 0.642452 & 0.64773 \\ 2 & 0.564769 & 0.578567 & 0.585707 & 0.576348 \\ 3 & 0.706261 & 0.59776 & 0.672737 & 0.658919 \\ 4 & 0.736114 & 0.61534 & 0.672584 & \mathbf{0 . 6 7 4 6 7 9} \\ 5 & 0.541579 & 0.47494 & 0.553948 & 0.523489 \\ & \mathbf{0 . 6 3 4 0 3 4} & 0.58918 & 0.625486 & \end{array}$

Table 7.2: InSiPS parameter testing results using the average aggregator in the fitness function. For each problem the best average fitness is bolded across the different parameter settings as well as across the different random seeds used. Parameter sets used: set 1: $p_{\text {_ccossover }}=0.45$, p_mutation $=0.45$; set $2:$ p_crossover $=0.30$, p_mutation $=0.60 ;$ set 3: p_crossover $=0.60, p_{-}$mutation $=0.30 ;$ set $4: p_{\text {c crossover }}$ $=0.75, p_{\text {_mutation }}=0.15$; set 5: $p_{\text {_crossover }}=0.15$, p_mutation $=0.75$. 


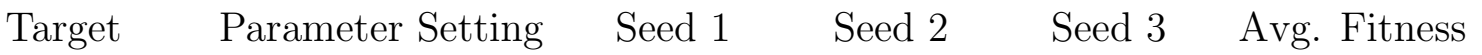

$\begin{array}{cccccc}\text { YAL054C } & & & & \\ & 1 & 0.356436 & 0.358372 & 0.325881 & \mathbf{0 . 3 4 6 8 9 6} \\ 2 & 0.285222 & 0.354912 & 0.289847 & 0.309994 \\ 3 & 0.330718 & 0.310009 & 0.342178 & 0.327635 \\ & 4 & 0.338123 & 0.316811 & 0.330111 & 0.328348 \\ \text { Avg Fitness } & 5 & 0.329336 & 0.292616 & 0.31709 & 0.313014 \\ & & \mathbf{0 . 3 2 7 9 6 7} & 0.326544 & 0.321021 & \end{array}$

YBR274W

Avg. Fitness

$\begin{array}{ccccc}1 & 0.443845 & 0.347881 & 0.371253 & 0.38766 \\ 2 & 0.296172 & 0.326578 & 0.312048 & 0.311599 \\ 3 & 0.360816 & 0.367954 & 0.37319 & 0.36732 \\ 4 & 0.422009 & 0.332688 & 0.42987 & \mathbf{0 . 3 9 4 8 5 6} \\ 5 & 0.28742 & 0.355942 & 0.343211 & 0.328858 \\ & 0.362052 & 0.346209 & \mathbf{0 . 3 6 5 9 1 4} & \end{array}$

YOL054W

Avg. Fitness

$\begin{array}{ccccc}1 & 0.361294 & 0.414785 & 0.410863 & 0.395647 \\ 2 & 0.354156 & 0.353143 & 0.390802 & 0.366034 \\ 3 & 0.392719 & 0.404747 & 0.342896 & 0.380121 \\ 4 & 0.407836 & 0.390262 & 0.416178 & \mathbf{0 . 4 0 4 7 5 9} \\ 5 & 0.358562 & 0.36304 & 0.315938 & 0.345847 \\ & 0.374913 & \mathbf{0 . 3 8 5 1 9 5} & 0.375335 & \end{array}$

Table 7.3: InSiPS parameter testing results using the maximum aggregator in the fitness function. For each problem the best average fitness is bolded across the different parameter settings as well as across the different random seeds used. Parameter sets used: set 1: p_crossover $=0.45, p_{\text {_mutation }}=0.45$; set 2: $p_{-}$crossover $=0.30$, $p_{-}$mutation $=0.60 ;$ set $3: p_{-}$crossover $=0.60, p_{-}$mutation $=0.30 ;$ set $4:$ p_crossover $=0.75, p_{-}$mutation $=0.15$; set $5:$ p_crossover $=0.15, p_{-}$mutation $=0.75$. 
When examining these results it can be seen that the fitness achieved varies similarly between random seeds as it does between using different parameter settings. For example, when using the maximum aggregator in the fitness function on problem YBR274W, parameter set 4 achieves nearly the best fitness from two different random seeds (fitnesses 0.4220 and 0.4299). However, using the third random seed produces one of the lowest fitness values among all combination of parameters and random seeds for that problem (fitness 0.3327). Similarly, using the average aggregator function, the effect of having a good random seed (Seed 1) can be seen. For any of the test problems, runs seeded with random seed 1 outperformed the others on average across the parameter sets. Overall, InSiPS seems to perform best with a relatively balanced parameter set. It should also be noted that the overall performance doesn't change drastically between different parameter sets when the different problems and random seeds are considered. The inherent stochastic nature of InSiPS' GA seems to provide a level of robustness, allowing any "bad" parameter set to be overcome. This stability over a variety of input parameters frees the user from having to perform lengthy parameter tuning experiments, due to the fact that InSiPS' performance doesn't vary significantly when the input parameters are changed.

\subsubsection{Wet-Lab Experimental Validation}

InSiPS was run on all of the 18 experimental candidates using the maximum aggregator as a part of the fitness function. A population size of 1,000 sequences was used with the following parameters: $p_{\_}$crossover $=0.5, p_{-}$mutation $=0.4, p_{\_}$copy $=0.1$, p_aa_mutation $=0.05$. InSiPS was run for a minimum of 250 generations at which point it continued running until a new best sequence wasn't found for 50 generations. The three target proteins for which InSiPS designed the fittest potential inhibitors were proteins YAL017W, YBL051C and YDL001W. Once they were identified, InSiPS was rerun on each of these targets three more times (each time with a different random seed) in the same manner. The fittest sequences generated from this second set of InSiPS runs (namely anti-YAL017W, anti-YBL051C and anti-YDL001W) were prepared for experimental validation. In all three cases the InSiPS designed protein

had a much higher predicted interaction score with the target protein than with the highest scoring non-target protein. When considering the average non-target PIPE 
score, the difference was even more pronounced. The respective "learning curves" for these three best runs are illustrated in Figure 7.11. For the fittest protein sequence generated by InSiPS at each generation this figure plots the predicted interaction scores (against the target, highest scoring non-target and average non-target score). This allows us to visualize how the quality of the best generated sequence improves as the generations progress in a given run of InSiPS.

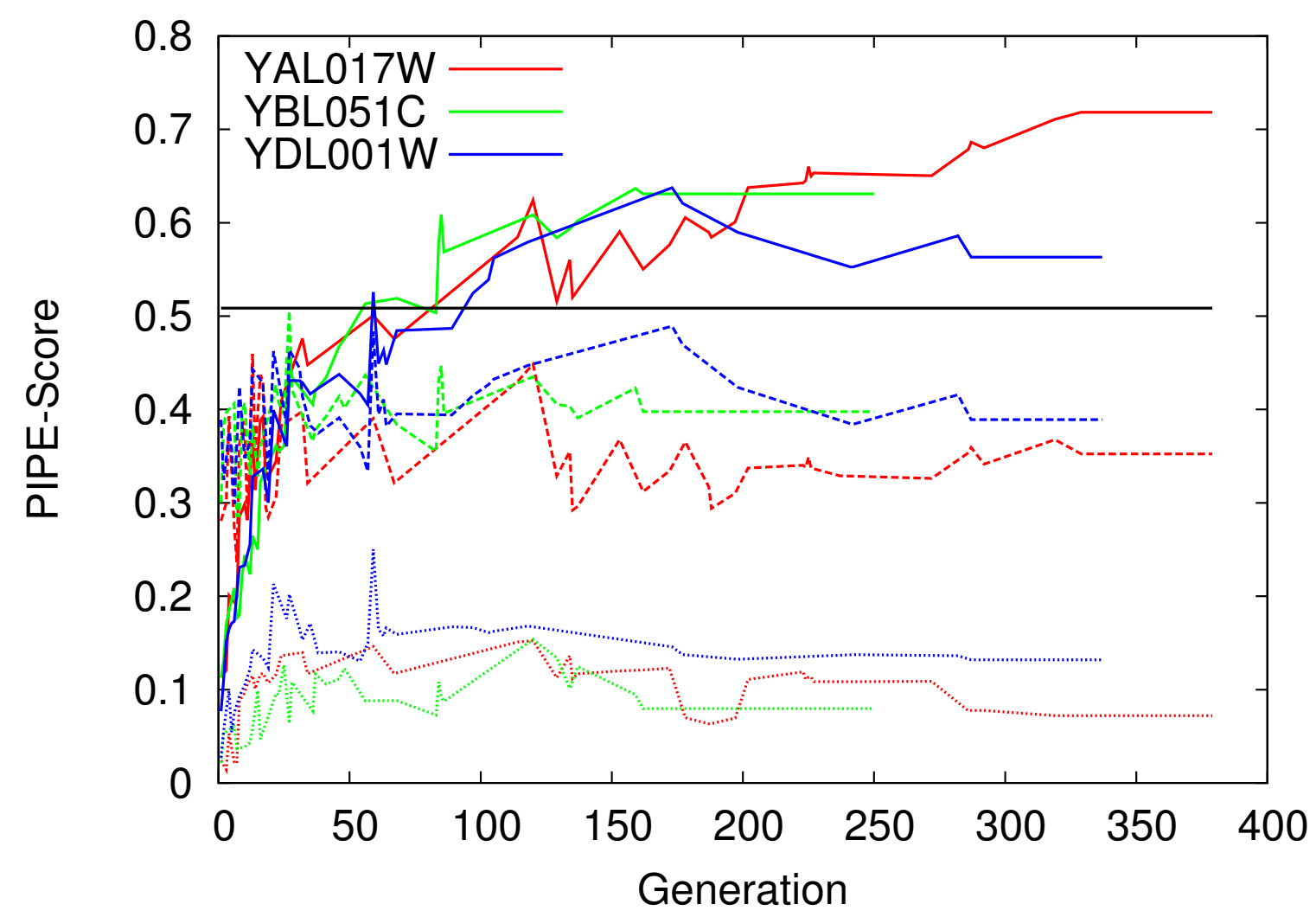

Figure 7.11: Fitness of the best generated sequence for each of the three experimental candidates over the generations of their respective InSiPS runs. In each generation the PIPE predicted interaction score of the fittest generated sequence is plotted against the target protein (solid line), the highest scoring non-target (dashed line) and the average non-target score (dotted line). The production of the synthetic anti-YAL017W, anti-YBL051C and anti-YDL001W are plotted in red, green and blue, respectively. The black line represents the threshold at which any protein pair would be predicted to interact (with a false positive rate of $<0.5 \%$ ). 
For each of the potentially inhibitory proteins generated by InSiPS, their respective coding DNA segments were commercially synthesized and cloned into an expression vector under the transcriptional control of a promoter. By doing this, the anti-target proteins generated by InSiPS could be expressed in yeast cells and their ability to bind to and inhibit their respective targets could be evaluated. Conditional sensitivity tests were conducted to validate the efficacy of the generated inhibitory proteins. As previously mentioned, one criterion for selecting the original set of potential experimental targets was that their deactivation or absence from the cell resulted in the cells sensitivity to certain stressors. This means that if the gene which codes for the protein in question was knocked-out (deleted), the cell would then exhibit increased sensitivity to certain environmental conditions (such as the presence of a chemical compound or damaging radiation). If the designed inhibitory proteins bind specifically to their targets, the cell should exhibit similar conditional sensitivities to the gene deletion strain. This would be due to the unnatural interaction between the target protein and the designed inhibitor which hinders the target protein from carrying out its normal duties in the cell.

These experiments were carried out in the following manner. First, four different strains of $S$. cerevisiae are used, namely the wild type control strain $(W T)$, a second control strain containing an empty plasmid vector $(W T+)$, a strain containing a plasmid which can induce the production of the InSiPS generated anti-target protein $(W T+\operatorname{InSiPS})$ and a strain in which the gene which codes for the target protein is knocked-out $(\Delta$ gene). In these tests, the first two strains are used as negative controls to show the reaction of the cell under certain conditions with the target protein uninhibited. The last strain is used as a positive control to show the effect of not having the target protein present under certain environmental conditions. If the InSiPS generated protein successfully binds specifically to the target protein then the strain producing this protein should resemble the gene knock out strain under a given condition. The desired result for these tests is to witness a decrease in viable cells (increased sensitivity) in the strains producing the generated anti-target proteins compared to the negative control strains under a certain condition (ie. it reacts similarly similarly to the gene deletion strain). The results of these tests are the number of viable cells left after exposure for each strain, given as a percent of the 
colony counts observed under normal conditions. Initial tests for the generated antiYDL001W protein sequence were not successful and therefore further experiments were cancelled. Details for the experiments on the InSiPS generated anti-YBL051C and anti-YAL017W are given below.

\section{Target: YBL051C}

For target protein YBL051C, the best run of InSiPS generated a synthetic protein sequence with a fitness of 0.3799 . Its predicted interaction score with the YBL051C was 0.6309 with a maximum and average off-target score of 0.3979 and 0.0797 , respectively. This generated protein produces a significant separation between the target and off-targets in terms of predicted interaction score.

When the gene which codes for YBL051C (PIN4) is deleted, it is known that $S$. cerevisiae becomes sensitive to cycloheximide, an inhibitor of biosynthesis. When even challenged with a low concentration of cycloheximide, this knock-out strain cannot generate new proteins as easily. In this test, all four experimental strains $(W T$, $W T+, W T+I n S i P S$ and $\triangle P I N 4)$ were exposed to $65 \mathrm{ng} / \mathrm{mL}$ of cycloheximide and then the number of living cells were counted on the basis of the colonies that were formed. A summary of five such experiments is given in Table 7.4 and the averages of these experiments are visualized in Figure 7.12.

As can be seen in the figure and table, the wild type strain and the strain containing the empty plasmid ( $W T$ and $W T+$ ) show a similar sensitivity to cyclohexamide. On the other hand, a large reduction in viable cells for both the $W T+\operatorname{In} S i P S$ strain and $\triangle P I N 4$ strain is observed. Although the decrease in cells in the $W T+\operatorname{InSiPS}$ strain isn't as dramatic as the positive control $\triangle P I N 4$ strain, the large reduction from the negative control strains is seen which suggests that the InSiPS generated

anti-YBL051C protein is successfully inhibiting YBL051C, therefore making the cell more sensitive to cyclohexamide. 


\begin{tabular}{ccccc} 
Run & WT & WT+ & WT + InSiPS & $\Delta$ PIN4 \\
\hline & & & & \\
1 & $86 \%$ & $88 \%$ & $52 \%$ & $24 \%$ \\
2 & $87 \%$ & $96 \%$ & $53 \%$ & $31 \%$ \\
3 & $90 \%$ & $95 \%$ & $58 \%$ & $29 \%$ \\
4 & $97 \%$ & $91 \%$ & $59 \%$ & $23 \%$ \\
5 & $91 \%$ & $87 \%$ & $55 \%$ & $28 \%$ \\
\hline \multirow{2}{*}{ Avg. } & $90 \%$ & $91 \%$ & $56 \%$ & $27 \%$ \\
\hline
\end{tabular}

Table 7.4: Colony counts for each of the four S. cerevisiae strains (WT: wild type; $W T+$ : wild type cells containing an empty plasmid; $W T+\operatorname{InSiPS}$ : wild type strain containing a plasmid that produces anti-YBL051C proteins; $\triangle P I N 4$ : a knockout strain for YBL051C) after exposure to $65 \mathrm{ng} / \mathrm{mL}$ of cycloheximide. Colony counts after exposure are normalized to the average colony counts observed under normal conditions.

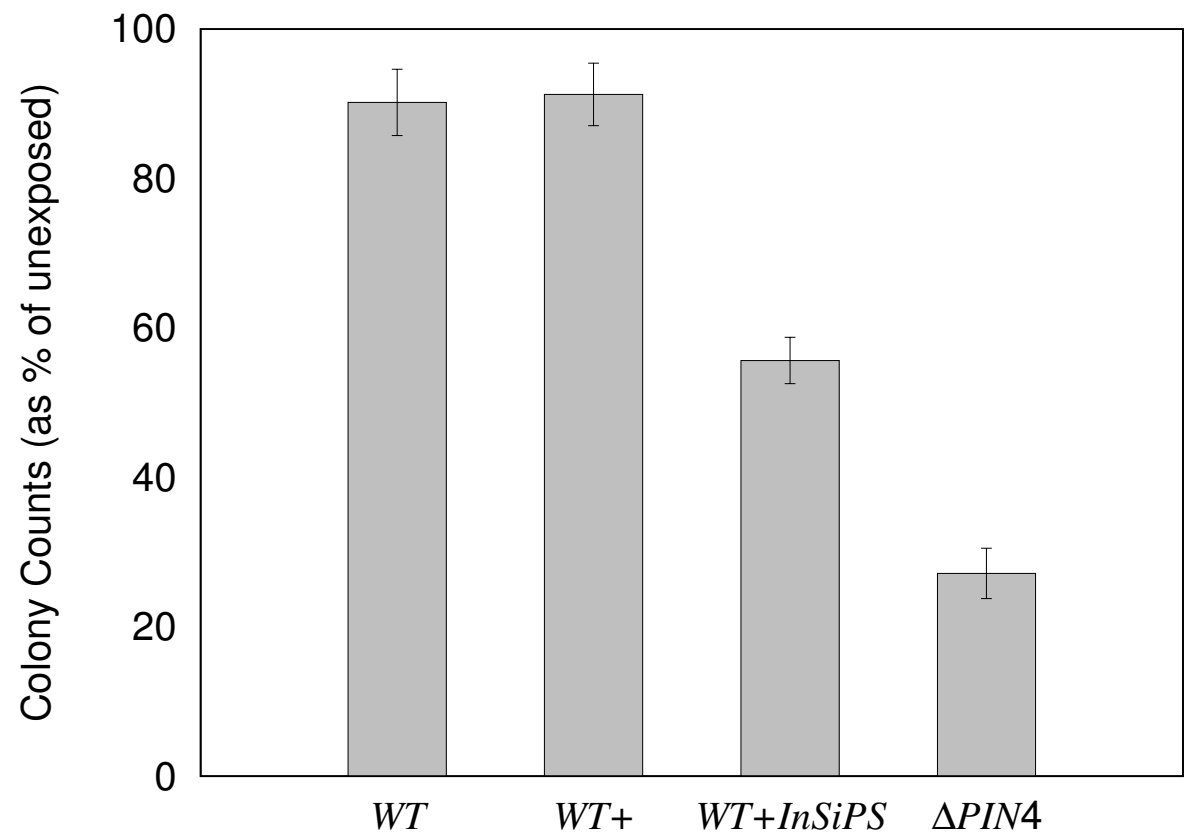

Figure 7.12: Average colony counts for each of the four S. cerevisiae strains (WT: wild type; $W T+$ : wild type cells containing an empty plasmid; $W T+\operatorname{InSiPS}$ : wild type strain containing a plasmid that produces anti-YBL051C proteins; $\triangle P I N 4$ : a knockout strain for YBL051C) after exposure to $65 \mathrm{ng} / \mathrm{mL}$ of cyclohexamide. Average colony counts after exposure are normalized to the average colony counts observed under normal conditions. Error bars represent standard deviation. 


\section{Target: YAL017W}

For target protein YAL017W, the best run of InSiPS generated a synthetic protein sequence with a fitness of 0.4652. Its predicted interaction score with the YAL017W was 0.7183 with a maximum and average off-target score of 0.3524 and 0.0721 , respectively. As seen in the previous example, this generated protein produces a significant separation between the target and off-targets in terms of predicted interaction score, meaning very few, if any, interactions between the InSiPS generated anti-YAL017W and any of of the non-target proteins would be expected.

When the gene which codes for YAL017W (PSK1) is deleted, it is known that S. cerevisiae becomes more sensitive to ultraviolet (UV) light. Without YAL017W, the cell's ability to repair the DNA damage caused by UV light exposure is severely diminished. In this test, all four experimental strains $(W T, W T+, W T+\operatorname{InSiPS}$ and $\triangle P I N 4)$ were exposed to UV light for 30 seconds then the number of living cells were counted on the basis of the colonies that were formed, as above, to determine the rate of cell survival (and therefore cell sensitivity to UV light). A summary of five such experiments is given in Table 7.5 and the averages of these experiments are visualized in Figure 7.13. On top of these tests, spot test analysis was also carried out and can be seen in Figure 7.14.

As in the previous example, the two negative control strains (WT and $W T+$ ) show similar sensitivity to the exposure of UV light. On the other hand a much larger reduction in viable cell count for both the $W T+\operatorname{InSiPS}$ strain and the positive control $\triangle P S K 1$ strain is observed. This drastic increase in UV sensitivity for the $W T+I n S i P S$ suggests that the InSiPS designed anti-YAL017W protein is successfully inhibiting YAL017W, which then reduces the cell's ability to recover from UV exposure, very much like the positive control $\triangle P S K 1$ strain. 


\begin{tabular}{|c|c|c|c|c|}
\hline Run & $W T$ & $W T+$ & $W T+\operatorname{InSiPS}$ & $\triangle P S K 1$ \\
\hline 1 & $54 \%$ & $51 \%$ & $20 \%$ & $13 \%$ \\
\hline 2 & $56 \%$ & $55 \%$ & $5 \%$ & $4 \%$ \\
\hline 3 & $53 \%$ & $56 \%$ & $8 \%$ & $5 \%$ \\
\hline 4 & $55 \%$ & $55 \%$ & $16 \%$ & $11 \%$ \\
\hline 5 & $57 \%$ & $56 \%$ & $17 \%$ & $14 \%$ \\
\hline Avg. & $55 \%$ & $54 \%$ & $14 \%$ & $10 \%$ \\
\hline
\end{tabular}

Table 7.5: Cell colony counts for each of the four $S$. cerevisiae strains (WT: wild type; $W T+$ : wild type cells containing an empty plasmid; $W T+\operatorname{InSiPS}$ : wild type strain containing a plasmid that produces anti-YAL017W proteins; $\triangle P S K 1$ : a knockout strain for YAL017W) after exposure to 30 seconds of ultraviolet light. Colony counts after exposure are normalized to the average colony counts observed under normal conditions.

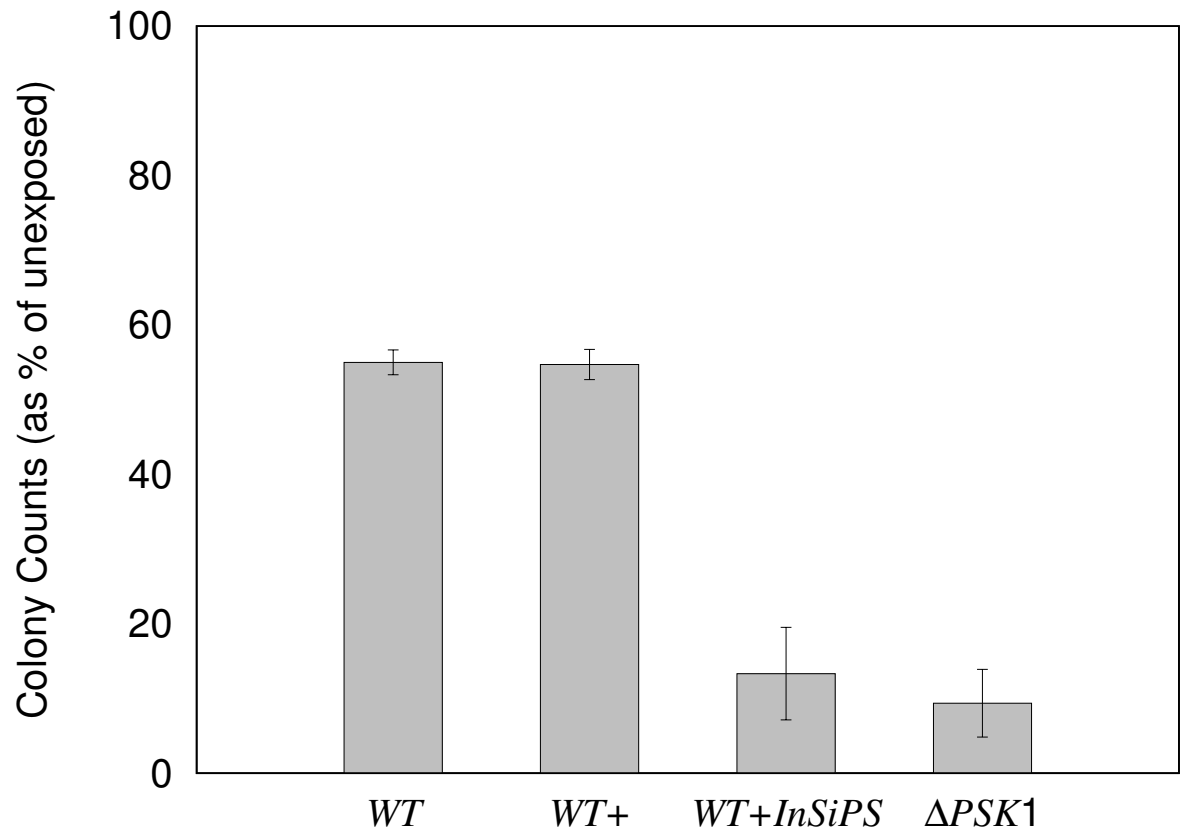

Figure 7.13: Average colony counts for each of the four S. cerevisiae strains (WT: wild type; $W T+$ : wild type cells containing an empty plasmid; $W T+\operatorname{InSiPS}$ : wild type strain containing a plasmid that produces anti-YAL017W proteins; $\triangle P S K 1$ : a knockout strain for YAL017W) after exposure to 30 seconds of ultraviolet light. Average colony counts after exposure are normalized to the average colony counts observed under normal conditions. Error bars represent standard deviation. 


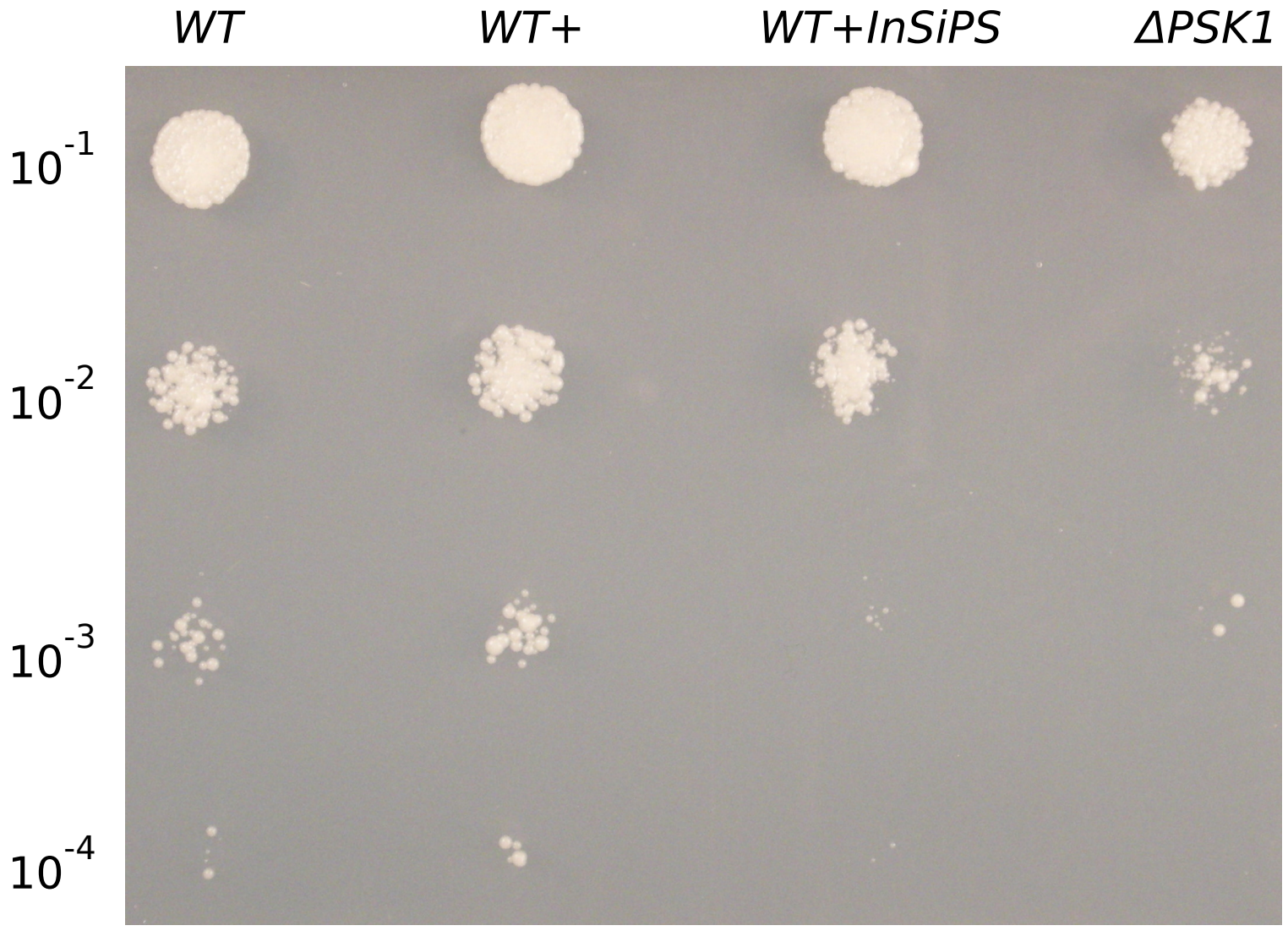

Figure 7.14: Spot test for the four $S$. cerevisiae strains (WT: wild type; $W T+$ : wild type cells containing an empty plasmid; $W T+\operatorname{InSiPS}$ : wild type strain containing a plasmid that produces anti-YAL017W proteins; $\triangle P S K 1$ : a knockout strain for YAL017W) grown for 48 hours after 30 seconds of exposure to ultraviolet light. Each column contains an equal number of cells diluted 10X down each row. Decreased growth in columns 3 and 4 indicates that the expression of anti-YAL017W sensitizes cells to UV in a similar manner as the absence of YAL017W. 


\section{Chapter 8}

\section{Conclusions and Future Work}

The Protein-protein Interaction Prediction Engine is a computational tool used to predict PPIs and formed the basis for the work presented here. The entire history of the PIPE project, before the novel work presented in this thesis, was discussed in Chapter 3. As discussed in that chapter, PIPE as a PPI prediction method outperformed all other tools (reviewed in Chapter 2) in terms of both applicability and predictive and computational performance. Over the course of this thesis contributions were made in four main areas relating to PIPE.

The first area of contribution consisted of further improvements of the main PIPE algorithm, as discussed in Chapter 4. Here the development of an add-on to PIPE used to predict the actual sties of interaction between proteins predicted to interact was detailed (PIPE-Sites, Section 4.1). In addition to this a new "similarityweighted" scoring function used to improve the predictive performance of PIPE as well as a significant computational enhancement was discussed (Section 4.2). Finally, all of these improvements were carefully integrated into the current implementation of PIPE, namely MP-PIPE2, as described in Section 4.3. In terms of further developments of the current PIPE implementation, there are no obvious issues to tackle to improve overall computational performance. This is largely due to the fact the MPPIPE2 can be deployed on any given compute cluster, scales well to use all available computational resources and can make nearly all PPI predictions in a fraction of a second. Improving PIPE's predictive performance (to achieve a higher sensitivity at the typical operation point, sensitivity of 99.95\%) is always a goal of PIPE developers. Research into this, especially for prokaryotic organisms such as Escherichia coli, is of high priority. E. coli is a highly studied model organism and high quality PPI predictions would be of value not only locally but to the scientific community as a whole. 
The second area of contribution discussed in this thesis was the various applications of PIPE and the analysis of the resulting data, described in Chapter 5. Section 5.1 described the use of PIPE to predict cross-species PPIs, which would be used in a few projects discussed later in the thesis. Sections 5.2 and 5.3 described the further analysis of the predicted $S$. cerevisiae and $H$. sapiens interactomes, respectively. In both of these sections novel data mined from the predicted interaction networks were shown to be biologically relevant and to have a wide variety of practical applications. Moving forward, there is still a great interest in high quality PPI predictions in other organisms. A current collaboration is underway with Agriculture Canada to study novel PPI predictions in soy bean with the goal of identifying novel proteins involved in flowering. The ultimate goal of the project is to spread the cultivation of soy beans to areas further north in Canada than is currently possible. For this to be possible they would like to produce a soy bean strain which will flower earlier than current strains to allow for cultivation in colder climates. To be able to achieve this goal, further information of the underlying pathways involved in flowering needs to be understood, where novel PPIs predicted by PIPE can be of enormous help. There is also interest in predicted host-pathogen PPIs between $H$. sapiens and viruses such as Hepatitis $\mathrm{C}$ and HIV. These predictions can be used to help understand the various mechanisms employed by the virus to infect and replicate within the host. Interest in predicted PPIs for other model organisms (including mouse, rat, rice, etc.) are also in demand. Data for these organisms are being collected and proteome-wide, all-to-all prediction runs are being planned. In parallel with this, a public database which will hold all of the PPIs predicted by PIPE is being developed. It is expected that this database, including all of the data described in this thesis, will be launched in early 2016.

The third area of contribution discussed in this thesis was the use of PIPE predicted interactomes to study the evolution of closely related organisms, discussed in Chapter 6. In Section 6.2, the evolutionary transition in individuality from unicellulatiry to multicellularity was studied in the volvocine family of green algae. Here various PPI network measures of modularity and integration were introduced and trends across the organisms studied were described. In Section 6.3, the study of five closely related yeast strains was described. Here a novel null model used to compare 
PPI networks was also introduced. In both cases, the value of studying PPIs and protein clusters was illustrated by showing the information gained over traditional sequence-based methods of comparison. Moving forward, the ideas presented here could be formalized into standard methods of comparing PPI networks. Also, further work on other sets of related organisms need to be done to see if the trends and general results arrived at in these sections hold true across other species.

Lastly, the final area of contribution described in this thesis was the In Silico Protein Synthesizer algorithm built to produce inhibitory proteins designed to interact with a given target protein while avoiding interaction with defined non-target proteins, described in Chapter 7. Wet-lab experiments suggest that this algorithm can successfully design inhibitory proteins. Currently InSiPS can only produce inhibitory proteins for S. cerevisiae. Moving forward, InSiPS will be expanded to be able to run in other organisms, including H. spaiens, however there are some implementation details which need to be settled. Specifically, the size of the unified database employed by InSiPS (which is loaded into main memory for every running InSiPS process) is significantly larger in other organisms (including an order of magnitude bigger for H. sapiens). This has obvious memory-usage implications which need to be resolved. A balance between memory-usage and overall computational efficiency will need to be achieved for InSiPS to be able to run on more complex organisms. Once this has been done, there are a variety of potential applications. One example would be within H. sapiens. Adult tissue stem cells are undifferentiated progenitors that have the capacity to preserve the homeostasis of tissues. They can maintain cell populations (eg. intestinal stem cells) [220], or repair tissues when they are injured (eg. muscle stem cells) [36]. Adult muscle stem cells participate in the regeneration of skeletal muscle and have the potential for the treatment of Duchenne muscular dystrophy [270]. There is hope that muscle stem cells from healthy donors could be isolated, expanded in vitro, and grafted in patients with muscular dystrophy. However, one challenge remains the expansion of the pool of stem cells, because in vitro culture pushes the cells towards premature differentiation and prevents them from restoring the stem cell void in the recipient patients [186]. A family of genes (SIX family), as well as the proteins they code for, are known to be involved with the processes of proliferation and differentiation of muscle stem cells [162, 161, 297]. InSiPS 
could be used to design a protein that would modulate or alter the function of SIX family proteins, such that in vitro proliferation of stem cells would be promoted, and premature differentiation blocked. This would allow the donor samples to last longer as undifferentiated stem cells, thereby facilitating the production of large numbers of cells to treat patients. Research into the application of InSiPS to this project is ongoing. Another use for InSiPS would be to design an inhibitory proteins used to interfere with host-pathogen PPIs. As discussed above, work is being done to produce predicted PPIs between $H$. sapiens and a variety of pathogens. Once novel PPIs are identified to help understand the various mechanisms at play with these pathogens, inhibitory proteins could be designed to interrupt these processes.

Assuming progress can be made on PIPE's ability to predict E. coli PPIs, there are a few opportunities to apply InSiPS there as well. Most strains of E. coli are harmless and actually perform a role in the normal functioning of the microbiota in the gut of their host. Some strains, however, are pathogenic and are usually transmitted through food, water or through contact with animals. One such strain is the Shiga toxin producing E.coli [27]. These strains are commonly heard about in the media in relation to food-based outbreaks. People exposed Shiga toxin-producing E. coli suffer from severe diarrhea and vomiting. The problem with these strains are that they are difficult to detect in food and current technologies are rather time consuming. InSiPS can be used here to design proteins which would act as molecular markers. These proteins would be designed to bind to proteins specific to the Shiga toxin-producing $E$. coli strains and would also carry physical properties that make them easy to detect once bound to their target. The benefit of such an approach is that all of the proteins in the harmless stains of E. coli (expected to be present) would be considered nontargets in the InSiPS design process. These designed proteins would then not bind to any non-toxic E. coli protein, resulting in a relatively easy Shiga toxin detection test that could be applied within the food production system. Another project is the study of antibiotic resistance in E. coli. A common mechanism of antibiotic resistance in many bacteria, including E. coli, is the use of efflux pumps [58]. These pumps (typically composed of three separate protein subunits) cause resistance by expelling antibiotics from the bacterium in question, ensuring they do not reach their intended target within the cell. Once InSiPS has been modified to allow for the production of 
synthetic E. coli proteins, the three proteins making up these efflux pumps become natural targets. An InSiPS produced synthetic protein would be expected to bind to its target in the pump, thus inhibiting it and interrupting the pump's function, leading to a decrease in antibiotic resistance. 


\section{Bibliography}

[1] T. Aas, a. L. Børresen, S. Geisler, B. Smith-Sørensen, H. Johnsen, J. E. Varhaug, L. A. Akslen, and P. E. Lønning. Specific P53 mutations are associated with de novo resistance to doxorubicin in breast cancer patients. Nature medicine, 2(7):811-814, jul 1996.

[2] F. Abe and K. Horikoshi. Tryptophan permease gene TAT2 confers highpressure growth in Saccharomyces cerevisiae. Molecular and cellular biology, 20(21):8093-102, nov 2000.

[3] B. Adamcsek, G. Palla, I. J. Farkas, I. Derényi, and T. Vicsek. CFinder: locating cliques and overlapping modules in biological networks. Bioinformatics (Oxford, England), 22(8):1021-3, apr 2006.

[4] Y.-Y. Ahn, J. P. Bagrow, and S. Lehmann. Link communities reveal multiscale complexity in networks. Nature, 466(7307):761-4, aug 2010.

[5] I. Albert and R. Albert. Conserved network motifs allow protein-protein interaction prediction. Bioinformatics (Oxford, England), 20(18):3346-52, dec 2004 .

[6] B. Alberts, A. Johnson, J. Lewis, M. Raff, K. Roberts, and P. Walter. Protein Function. In Molecular Biology of the Cell. Garland Science, New York, NY, 4 edition, 2002.

[7] P. Aloy and R. B. Russell. Interrogating protein interaction networks through structural biology. Proceedings of the National Academy of Sciences of the United States of America, 99(9):5896-901, apr 2002.

[8] P. Aloy and R. B. Russell. InterPreTS: protein Interaction Prediction through Tertiary Structure. Bioinformatics, 19(1):161-162, jan 2003.

[9] A. Amos-Binks. Proteome-scale Protein-protein Interaction Site Prediction and Novel Motif Discovery Using Re-occurring Polypeptide Sequences. Master of applied science, Carleton University, 2010.

[10] A. Amos-Binks, C. Patulea, S. Pitre, A. Schoenrock, Y. Gui, J. R. Green, A. Golshani, and F. Dehne. Binding site prediction for protein-protein interactions and novel motif discovery using re-occurring polypeptide sequences. BMC bioinformatics, 12(1):225, jan 2011.

[11] E. Andres Leon, I. Ezkurdia, B. García, A. Valencia, and D. Juan. EcID. A database for the inference of functional interactions in E. coli. Nucleic acids research, 37(Database issue):D629-35, jan 2009. 
[12] M. R. Arkin and J. a. Wells. Small-molecule inhibitors of protein-protein interactions: progressing towards the dream. Nature reviews. Drug discovery, 3(4):301-17, apr 2004.

[13] W. S. Armbruster, C. Pélabon, G. H. Bolstad, and T. F. Hansen. Integrated phenotypes: understanding trait covariation in plants and animals. Philosophical transactions of the Royal Society of London. Series B, Biological sciences, 369(1649):20130245, aug 2014.

[14] a. S. Aytuna, A. Gursoy, and O. Keskin. Prediction of protein-protein interactions by combining structure and sequence conservation in protein interfaces. Bioinformatics (Oxford, England), 21(12):2850-5, jun 2005.

[15] M. Babu, J. Vlasblom, S. Pu, X. Guo, C. Graham, B. D. M. Bean, H. E. Burston, F. J. Vizeacoumar, J. Snider, S. Phanse, V. Fong, Y. Y. C. Tam, M. Davey, O. Hnatshak, N. Bajaj, S. Chandran, T. Punna, C. Christopolous, V. Wong, A. Yu, G. Zhong, J. Li, I. Stagljar, E. Conibear, S. J. Wodak, A. Emili, and J. F. Greenblatt. Interaction landscape of membrane-protein complexes in Saccharomyces cerevisiae. Nature, 489(7417):585-589, sep 2012.

[16] A. Baspinar, E. Cukuroglu, R. Nussinov, O. Keskin, and A. Gursoy. PRISM: a web server and repository for prediction of protein-protein interactions and modeling their 3D complexes. Nucleic acids research, 42(Web Server issue):W285-9, jul 2014.

[17] C. Belloch, S. Orlic, E. Barrio, and A. Querol. Fermentative stress adaptation of hybrids within the Saccharomyces sensu stricto complex. International journal of food microbiology, 122(1-2):188-95, feb 2008.

[18] A. Ben-Hur and W. S. Noble. Kernel methods for predicting protein-protein interactions. Bioinformatics (Oxford, England), 21 Suppl 1:i38-46, jun 2005.

[19] A. Ben-Hur and W. S. Noble. Choosing negative examples for the prediction of protein-protein interactions. BMC bioinformatics, 7 Suppl 1:S2, jan 2006.

[20] P. Benjamin Stranges and B. Kuhlman. A comparison of successful and failed protein interface designs highlights the challenges of designing buried hydrogen bonds. Protein Science, 22:74-82, 2013.

[21] S. L. Berg JM, Tymoczko JL. Eukaryotic Protein Synthesis Differs from Prokaryotic Protein Synthesis Primarily in Translation Initiation. In Biochemistry. 5th edition, page http://www.ncbi.nlm.nih.gov/books/NBK22531/. W H Freeman, New York, NY, 2002.

[22] S. Binny Priya, S. Saha, R. Anishetty, and S. Anishetty. A matrix based algorithm for Protein-Protein Interaction prediction using Domain-Domain Associations. Journal of theoretical biology, 326:36-42, jun 2013. 
[23] A. Birlutiu, F. D'Alche-Buc, and T. Heskes. A Bayesian Framework for Combining Protein and Network Topology Information for Predicting Protein-Protein Interactions. IEEE/ACM transactions on computational biology and bioinformatics / IEEE, ACM, 12(3):538-50, may 2015.

[24] J. R. Bock and D. A. Gough. Predicting protein-protein interactions from primary structure. Bioinformatics (Oxford, England), 17(5):455-60, may 2001.

[25] J. R. Bock and D. A. Gough. Whole-proteome interaction mining. Bioinformatics, 19(1):125-134, jan 2003.

[26] A. Bonvin. Coming to peace with protein complexes? 5th CAPRI evaluation meeting, April 17-19th 2013-Utrecht. Proteins, 81(12):2073-4, dec 2013.

[27] A. Bryan, I. Youngster, and A. J. McAdam. Shiga Toxin Producing Escherichia coli. Clinics in laboratory medicine, 35(2):247-72, jun 2015.

[28] L. Burger and E. van Nimwegen. Accurate prediction of protein-protein interactions from sequence alignments using a Bayesian method. Molecular systems biology, 4(165):165, jan 2008.

[29] G. Butland, M. Babu, J. J. Díaz-Mejía, F. Bohdana, S. Phanse, B. Gold, W. Yang, J. Li, A. G. Gagarinova, O. Pogoutse, H. Mori, B. L. Wanner, H. Lo, J. Wasniewski, C. Christopolous, M. Ali, P. Venn, A. Safavi-Naini, N. Sourour, S. Caron, J.-Y. Choi, L. Laigle, A. Nazarians-Armavil, A. Deshpande, S. Joe, K. a. Datsenko, N. Yamamoto, B. J. Andrews, C. Boone, H. Ding, B. Sheikh, G. Moreno-Hagelseib, J. F. Greenblatt, and A. Emili. eSGA: E. coli synthetic genetic array analysis. Nature methods, 5(9):789-795, 2008.

[30] G. Butland, J. M. Peregrín-Alvarez, J. Li, W. Yang, X. Yang, V. Canadien, A. Starostine, D. Richards, B. Beattie, N. Krogan, M. Davey, J. Parkinson, J. Greenblatt, and A. Emili. Interaction network containing conserved and essential protein complexes in Escherichia coli. Nature, 433(7025):531-537, feb 2005.

[31] A. A. Caudy, Y. Guan, Y. Jia, C. Hansen, C. DeSevo, A. P. Hayes, J. Agee, J. R. Alvarez-Dominguez, H. Arellano, D. Barrett, C. Bauerle, N. Bisaria, P. H. Bradley, J. S. Breunig, E. Bush, D. Cappel, E. Capra, W. Chen, J. Clore, P. A. Combs, C. Doucette, O. Demuren, P. Fellowes, S. Freeman, E. Frenkel, D. Gadala-Maria, R. Gawande, D. Glass, S. Grossberg, A. Gupta, L. Hammonds-Odie, A. Hoisos, J. Hsi, Y.-H. H. Hsu, S. Inukai, K. J. Karczewski, X. Ke, M. Kojima, S. Leachman, D. Lieber, A. Liebowitz, J. Liu, Y. Liu, T. Martin, J. Mena, R. Mendoza, C. Myhrvold, C. Millian, S. Pfau, S. Raj, M. Rich, J. Rokicki, W. Rounds, M. Salazar, M. Salesi, R. Sharma, S. Silverman, C. Singer, S. Sinha, M. Staller, P. Stern, H. Tang, S. Weeks, M. Weidmann, A. Wolf, C. Young, J. Yuan, C. Crutchfield, M. McClean, C. T. Murphy, M. Llinás, D. Botstein, O. G. Troyanskaya, and M. J. Dunham. A new 
system for comparative functional genomics of Saccharomyces yeasts. Genetics, 195(1):275-87, sep 2013.

[32] R. Cazabet, F. Amblard, and C. Hanachi. Detection of Overlapping Communities in Dynamical Social Networks. In 2010 IEEE Second International Conference on Social Computing, pages 309-314. IEEE, aug 2010.

[33] A. Ceol, A. Chatr-aryamontri, E. Santonico, R. Sacco, L. Castagnoli, and G. Cesareni. DOMINO: a database of domain-peptide interactions. Nucleic acids research, 35(Database issue):D557-60, jan 2007.

[34] D. T.-H. Chang, Y.-T. Syu, and P.-C. Lin. Predicting the protein-protein interactions using primary structures with predicted protein surface. BMC bioinformatics, 11 Suppl 1:S3, jan 2010.

[35] D. T.-H. Chang, Y.-T. Syu, and P.-C. Lin. Predicting the protein-protein interactions using primary structures with predicted protein surface. BMC bioinformatics, 11 Suppl 1(Suppl 1):S3, 2010.

[36] N. C. Chang and M. A. Rudnicki. Satellite cells: the architects of skeletal muscle. Current topics in developmental biology, 107(5):161-81, may 2014.

[37] A. Chatr-Aryamontri, B.-J. Breitkreutz, R. Oughtred, L. Boucher, S. Heinicke, D. Chen, C. Stark, A. Breitkreutz, N. Kolas, L. O'Donnell, T. Reguly, J. Nixon, L. Ramage, A. Winter, A. Sellam, C. Chang, J. Hirschman, C. Theesfeld, J. Rust, M. S. Livstone, K. Dolinski, and M. Tyers. The BioGRID interaction database: 2015 update. Nucleic acids research, 43(Database issue):D470-8, 2015 .

[38] P. Chatterjee, S. Basu, M. Kundu, M. Nasipuri, and D. Plewczynski. PPI_SVM: prediction of protein-protein interactions using machine learning, domaindomain affinities and frequency tables. Cellular $\& 3$ molecular biology letters, 16(2):264-78, jun 2011.

[39] J. Chen, B. J. Aronow, and A. G. Jegga. Disease candidate gene identification and prioritization using protein interaction networks. BMC bioinformatics, 10:73, jan 2009 .

[40] J. Chen, W. Hsu, M. L. Lee, and S.-K. Ng. Increasing confidence of protein interactomes using network topological metrics. Bioinformatics (Oxford, England), 22(16):1998-2004, aug 2006.

[41] L. Chen, L.-Y. Wu, Y. Wang, and X.-S. Zhang. Inferring protein interactions from experimental data by association probabilistic method. Proteins, 62(4):833-7, mar 2006. 
[42] P.-Y. Chen, C. M. Deane, and G. Reinert. Predicting and validating protein interactions using network structure. PLoS computational biology, 4(7):e1000118, jan 2008.

[43] W. Chen, Z. Liu, X. Sun, and Y. Wang. A game-theoretic framework to identify overlapping communities in social networks. Data Mining and Knowledge Discovery, 21(2):224-240, jul 2010.

[44] X.-w. Chen and J. C. Jeong. Sequence-based prediction of protein interaction sites with an integrative method. Bioinformatics (Oxford, England), 25(5):58591, mar 2009.

[45] X.-W. Chen and M. Liu. Prediction of protein-protein interactions using random decision forest framework. Bioinformatics (Oxford, England), 21(24):4394-400, $\operatorname{dec} 2005$.

[46] N. Chepelev, L. Chepelev, M. Alamgir, and A. Golshani. Large-Scale ProteinProtein Interaction Detection Approaches: Past, Present and Future. Biotechnology \&3 Biotechnological Equipment, 22(1):513-529, apr 2014.

[47] E. Chesler and M. Langston. Combinatorial Genetic Regulatory Network Analysis Tools for High Throughput Transcriptomic Data. In E. Eskin, T. Ideker, B. Raphael, and C. Workman, editors, Systems Biology and Regulatory Genomics, volume 4023 of Lecture Notes in Computer Science, pages 150-65. Springer Berlin Heidelberg, Berlin, Heidelberg, 2006.

[48] R. A. Chica. Protein engineering in the 21st century. Protein science : a publication of the Protein Society, 24(4):431-3, 2015.

[49] R. Chiva, M. López-Malo, Z. Salvadó, A. Mas, and J. M. Guillamón. Analysis of low temperature-induced genes (LTIG) in wine yeast during alcoholic fermentation. FEMS Yeast Research, 12(7):831-843, 2012.

[50] K.-C. Chou and Y.-D. Cai. Predicting protein-protein interactions from sequences in a hybridization space. Journal of proteome research, 5(2):316-22, feb 2006 .

[51] J. Clune, J.-B. Mouret, and H. Lipson. The evolutionary origins of modularity. Proceedings. Biological sciences / The Royal Society, 280(1755):20122863, mar 2013.

[52] G. M. Cooper. Regulation of Protein Function. In The Cell: A Molecular Approach. Sinauer Associates, Sunderland, MA, 2 edition, 2000.

[53] T. Dandekar, B. Snel, M. Huynen, and P. Bork. Conservation of gene order: a fingerprint of proteins that physically interact. Trends in biochemical sciences, 23(9):324-8, sep 1998. 
[54] L. Danon, A. Díaz-Guilera, J. Duch, and A. Arenas. Comparing community structure identification. Journal of Statistical Mechanics: Theory and Experiment, 2005(09):P09008-P09008, sep 2005.

[55] M. Dayhoff, R. Schwartz, and B. Orcutt. A model of evolutionary change in proteins. In Atlas of protein sequence and structure, pages 345-352. National Biomedical Research Foundation, Washington, DC, 1978.

[56] R. C. Deed, N. K. Deed, and R. C. Gardner. Transcriptional response of Saccharomyces cerevisiae to low temperature during wine fermentation. Antonie van Leeuwenhoek, 107(4):1029-48, 2015.

[57] W. F. DeGrado, Z. R. Wasserman, and J. D. Lear. Protein design, a minimalist approach. Science (New York, N.Y.), 243(4891):622-8, feb 1989.

[58] J. a. Delmar, C.-C. Su, and E. W. Yu. Bacterial Multidrug Efflux Transporters. Annual Review of Biophysics, 43(1):93-117, may 2014.

[59] M. Deng, S. Mehta, F. Sun, and T. Chen. Inferring domain-domain interactions from protein-protein interactions. Genome research, 12(10):1540-8, oct 2002.

[60] Y. Deng, L. Gao, and B. Wang. ppiPre: predicting protein-protein interactions by combining heterogeneous features. BMC systems biology, 7 Suppl 2(Suppl 2):S8, 2013.

[61] R. V. Devi, S. S. Sathya, and M. S. Coumar. Evolutionary algorithms for de novo drug design A survey. Applied Soft Computing, 27:543-552, feb 2015.

[62] Z. Dezso, Y. Nikolsky, T. Nikolskaya, J. Miller, D. Cherba, C. Webb, and A. Bugrim. Identifying disease-specific genes based on their topological significance in protein networks. BMC systems biology, 3:36, jan 2009.

[63] W. F. Doolittle. Is junk DNA bunk? A critique of ENCODE. Proceedings of the National Academy of Sciences of the United States of America, 110(14):5294300, apr 2013.

[64] L. Duncan, I. Nishii, A. Harryman, S. Buckley, A. Howard, N. R. Friedman, and S. M. Miller. The VARL gene family and the evolutionary origins of the master cell-type regulatory gene, regA, in Volvox carteri. Journal of molecular evolution, 65(1):1-11, jul 2007.

[65] J. Dutkowski and J. Tiuryn. A probabilistic model of neutral and selective dynamics of protein network evolution. Journal of computational biology : a journal of computational molecular cell biology, 20(9):631-42, sep 2013.

[66] M. D. Dyer, T. M. Murali, and B. W. Sobral. Computational prediction of host-pathogen protein-protein interactions. Bioinformatics (Oxford, England), 23(13):i159-66, jul 2007. 
[67] J. Eblen, I. Gerling, J. Saxton, J. Snoddy, and M. Langston. Graph Algorithms for Integrated Biological Analysis, with Applications to Type 1 Diabetes Data. In S. Butenko, W. A. Chaovalitwongse, and P. M. Pardalos, editors, Clustering Challenges in Biological Networks, pages 207-22. World Scientific Publishing Co. Pte. Ltd., 2009.

[68] S. R. Eddy. The ENCODE project: missteps overshadowing a success. Current biology : $C B, 23(7): \mathrm{R} 259-61$, apr 2013.

[69] a. J. Enright, I. Iliopoulos, N. C. Kyrpides, and C. a. Ouzounis. Protein interaction maps for complete genomes based on gene fusion events. Nature, 402(6757):86-90, nov 1999.

[70] a. J. Enright and C. a. Ouzounis. Functional associations of proteins in entire genomes by means of exhaustive detection of gene fusions. Genome biology, 2(9):RESEARCH0034, jan 2001.

[71] J. Ereño-Orbea, I. Oyenarte, and L. A. Martínez-Cruz. CBS domains: Ligand binding sites and conformational variability. Archives of biochemistry and biophysics, 540(1-2):70-81, dec 2013.

[72] J. Espadaler, O. Romero-Isart, R. M. Jackson, and B. Oliva. Prediction of protein-protein interactions using distant conservation of sequence patterns and structure relationships. Bioinformatics (Oxford, England), 21(16):3360-8, aug 2005 .

[73] T. S. Evans. Clique graphs and overlapping communities. Journal of Statistical Mechanics: Theory and Experiment, 2010(12):P12037, dec 2010.

[74] T. S. Evans and R. Lambiotte. Line Graphs, Link Partitions and Overlapping Communities. Physical Review E, 80(1):9, mar 2009.

[75] P. Fariselli, F. Pazos, A. Valencia, and R. Casadio. Prediction of protein-protein interaction sites in heterocomplexes with neural networks. European journal of biochemistry / FEBS, 269(5):1356-61, mar 2002.

[76] P. Fariselli, A. Zauli, I. Rossi, M. Finelli, P. Martelli, and R. Casadio. A neural network method to improve prediction of protein-protein interaction sites in heterocomplexes. In 2003 IEEE XIII Workshop on Neural Networks for Signal Processing (IEEE Cat. No.03TH8718), pages 33-41. IEEE, 2003.

[77] I. Farkas, D. Ábel, G. Palla, and T. Vicsek. Weighted network modules. New Journal of Physics, 9(6):180-180, jun 2007.

[78] I. Feldman, A. Rzhetsky, and D. Vitkup. Network properties of genes harboring inherited disease mutations. Proceedings of the National Academy of Sciences of the United States of America, 105(11):4323-8, mar 2008. 
[79] S. Fields and O. Song. A novel genetic system to detect protein-protein interactions. Nature, 340(6230):245-6, jul 1989.

[80] R. D. Finn, A. Bateman, J. Clements, P. Coggill, R. Y. Eberhardt, S. R. Eddy, A. Heger, K. Hetherington, L. Holm, J. Mistry, E. L. L. Sonnhammer, J. Tate, and M. Punta. Pfam: the protein families database. Nucleic acids research, 42(Database issue):D222-30, jan 2014.

[81] W. Fletcher and Z. Yang. INDELible: a flexible simulator of biological sequence evolution. Molecular biology and evolution, 26(8):1879-88, aug 2009.

[82] S. Fortunato. Community detection in graphs. Physics Reports, 486(3-5):75174, feb 2010.

[83] E. Fossum, C. C. Friedel, S. V. Rajagopala, B. Titz, A. Baiker, T. Schmidt, T. Kraus, T. Stellberger, C. Rutenberg, S. Suthram, S. Bandyopadhyay, D. Rose, A. von Brunn, M. Uhlmann, C. Zeretzke, Y.-A. Dong, H. Boulet, M. Koegl, S. M. Bailer, U. Koszinowski, T. Ideker, P. Uetz, R. Zimmer, and J. Haas. Evolutionarily Conserved Herpesviral Protein Interaction Networks. PLoS Pathogens, 5(9):e1000570, sep 2009.

[84] G. Franzot and O. Carugo. Computational approaches to protein-protein interaction. Journal of structural and functional genomics, 4(4):245-55, jan 2003.

[85] L. Fu, B. Niu, Z. Zhu, S. Wu, and W. Li. CD-HIT: accelerated for clustering the next-generation sequencing data. Bioinformatics (Oxford, England), 28(23):3150-2, dec 2012.

[86] A.-C. Gingras, R. Aebersold, and B. Raught. Advances in protein complex analysis using mass spectrometry. The Journal of physiology, 563(Pt 1):11-21, feb 2005.

[87] C. S. Goh, a. a. Bogan, M. Joachimiak, D. Walther, and F. E. Cohen. Coevolution of proteins with their interaction partners. Journal of molecular biology, 299(2):283-93, jun 2000.

[88] K.-I. Goh, M. E. Cusick, D. Valle, B. Childs, M. Vidal, and A.-L. Barabási. The human disease network. Proceedings of the National Academy of Sciences of the United States of America, 104(21):8685-90, may 2007.

[89] S. M. Gomez, W. S. Noble, and A. Rzhetsky. Learning to predict proteinprotein interactions from protein sequences. Bioinformatics, 19(15):1875-1881, oct 2003 .

[90] D. M. Goodstein, S. Shu, R. Howson, R. Neupane, R. D. Hayes, J. Fazo, T. Mitros, W. Dirks, U. Hellsten, N. Putnam, and D. S. Rokhsar. Phytozome: a comparative platform for green plant genomics. Nucleic acids research, 40(Database issue):D1178-86, jan 2012. 
[91] D. Graur, Y. Zheng, N. Price, R. B. R. Azevedo, R. a. Zufall, and E. Elhaik. On the immortality of television sets: "function" in the human genome according to the evolution-free gospel of ENCODE. Genome biology and evolution, 5(3):57890, jan 2013.

[92] S. Gregory. Finding overlapping communities in networks by label propagation. New Journal of Physics, 12(10):103018, oct 2010.

[93] M. R. Guarracino, A. Nebbia, V. Manna, A. Chinchuluun, and P. M. Pardalos. Efficient Prediction of Protein-Protein Interactions Using Sequence Information. In 2010 International Conference on Complex, Intelligent and Software Intensive Systems, pages 677-682. IEEE, feb 2010.

[94] Y. Gui. Prediction and Analysis of Novel Interaction Motifs in Yeast. Master of science, Carleton University, 2012.

[95] Y. Guo, M. Li, X. Pu, G. Li, X. Guang, W. Xiong, and J. Li. PRED_PPI: a server for predicting protein-protein interactions based on sequence data with probability assignment. BMC research notes, 3:145, jan 2010.

[96] Y. Guo, L. Yu, Z. Wen, and M. Li. Using support vector machine combined with auto covariance to predict protein-protein interactions from protein sequences. Nucleic acids research, 36(9):3025-30, may 2008.

[97] J. M. Hall, M. K. Lee, B. Newman, J. E. Morrow, L. a. Anderson, B. Huey, and M. C. King. Linkage of early-onset familial breast cancer to chromosome 17q21. Science (New York, N.Y.), 250(4988):1684-1689, dec 1990.

[98] T. Hamp and B. Rost. Evolutionary profiles improve protein-protein interaction prediction from sequence. Bioinformatics (Oxford, England), 31(12):1945-50, 2015.

[99] D. Han, H.-S. Kim, J. Seo, and W. Jang. A domain combination based probabilistic framework for protein-protein interaction prediction. Genome informatics. International Conference on Genome Informatics, 14:250-9, jan 2003.

[100] D.-S. Han, H.-S. Kim, W.-H. Jang, S.-D. Lee, and J.-K. Suh. PreSPI: a domain combination based prediction system for protein-protein interaction. Nucleic acids research, 32(21):6312-20, jan 2004.

[101] D.-S. Han, H.-S. Kim, W.-H. Jang, S.-D. Lee, and J. K. Suh. PreSPI: design and implementation of protein-protein interaction prediction service system. Genome informatics. International Conference on Genome Informatics, 15(2):171-80, jan 2004.

[102] S. Hanks, A. Quinn, and T. Hunter. The protein kinase family: conserved features and deduced phylogeny of the catalytic domains. Science, 241(4861):4252, jul 1988. 
[103] E. R. Hanschen, T. N. Marriage, P. J. Ferris, T. Hamaji, A. Toyoda, A. Fujiyama, V. Luria, H. Noguchi, Y. Minakuchi, M. Suzuki, H. Kawai-Toyooka, D. R. Smith, H. Sparks, J. Anderson, R. Neme, M. Kirschner, P. M. Durand, R. E. Michod, H. Nozaki, and B. J. S. C. Olson. Early Evolution of Cell Cycle Regulated Multicellular Development and Group Level Adaptations. To appear in Nature Genetics, 2015.

[104] T. F. Hansen. Is modularity necessary for evolvability? Remarks on the relationship between pleiotropy and evolvability. Bio Systems, 69(2-3):83-94, may 2003.

[105] D. G. Hardie. AMP-activated/SNF1 protein kinases: conserved guardians of cellular energy. Nature Reviews Molecular Cell Biology, 8(10):774-785, oct 2007.

[106] P. C. Havugimana, G. T. Hart, T. Nepusz, H. Yang, A. L. Turinsky, Z. Li, P. I. Wang, D. R. Boutz, V. Fong, S. Phanse, M. Babu, S. A. Craig, P. Hu, C. Wan, J. Vlasblom, V.-u.-N. Dar, A. Bezginov, G. W. Clark, G. C. Wu, S. J. Wodak, E. R. Tillier, A. Paccanaro, E. M. Marcotte, and A. Emili. A Census of Human Soluble Protein Complexes. Cell, 150(5):1068-1081, aug 2012.

[107] S. Henikoff and J. G. Henikoff. Amino acid substitution matrices from protein blocks. Proceedings of the National Academy of Sciences of the United States of America, 89(22):10915-9, nov 1992.

[108] M. D. Herron, J. D. Hackett, F. O. Aylward, and R. E. Michod. Triassic origin and early radiation of multicellular volvocine algae. Proceedings of the National Academy of Sciences of the United States of America, 106(9):3254-8, mar 2009.

[109] M. D. Herron and R. E. Michod. Evolution of complexity in the volvocine algae: transitions in individuality through Darwin's eye. Evolution; international journal of organic evolution, 62(2):436-51, feb 2008.

[110] C. Hsin Liu, K.-C. Li, and S. Yuan. Human protein-protein interaction prediction by a novel sequence-based co-evolution method: co-evolutionary divergence. Bioinformatics (Oxford, England), 29(1):92-8, jan 2013.

[111] L. Hu and K. Chan. Discovering Variable-Length Patterns in Protein Sequences for Protein-Protein Interaction Prediction. IEEE transactions on nanobioscience, 14(4):409-416, 2015.

[112] H. Huang and J. S. Bader. Precision and recall estimates for two-hybrid screens. Bioinformatics (Oxford, England), 25(3):372-8, feb 2009.

[113] S.-Y. Huang. Search strategies and evaluation in protein-protein docking: principles, advances and challenges. Drug Discovery today, 19(8):1081-1096, aug 2014. 
[114] M. Hue, M. Riffle, J.-P. Vert, and W. S. Noble. Large-scale prediction of proteinprotein interactions from structures. BMC bioinformatics, 11:144, jan 2010.

[115] S. Hunter, R. Apweiler, T. K. Attwood, A. Bairoch, A. Bateman, D. Binns, P. Bork, U. Das, L. Daugherty, L. Duquenne, R. D. Finn, J. Gough, D. Haft, N. Hulo, D. Kahn, E. Kelly, A. Laugraud, I. Letunic, D. Lonsdale, R. Lopez, M. Madera, J. Maslen, C. McAnulla, J. McDowall, J. Mistry, A. Mitchell, N. Mulder, D. Natale, C. Orengo, A. F. Quinn, J. D. Selengut, C. J. a. Sigrist, M. Thimma, P. D. Thomas, F. Valentin, D. Wilson, C. H. Wu, and C. Yeats. InterPro: the integrative protein signature database. Nucleic acids research, 37(Database issue):D211-5, jan 2009.

[116] C.-L. Hwang and A. S. M. Masud. Multiple Objective Decision Making Methods and Applications, volume 164 of Lecture Notes in Economics and Mathematical Systems. Springer Berlin Heidelberg, Berlin, Heidelberg, 1979.

[117] H. Hwang, T. Vreven, J. Janin, and Z. Weng. Protein-protein docking benchmark version 4.0. Proteins, 78(15):3111-4, nov 2010.

[118] T. Ideker and N. J. Krogan. Differential network biology. Molecular systems biology, 8(565):565, jan 2012.

[119] I. Iliopoulos, A. J. Enright, P. Poullet, and C. a. Ouzounis. Mapping functional associations in the entire genome of Drosophila melanogaster using fusion analysis. Comparative and functional genomics, 4(3):337-41, jan 2003.

[120] T. Ito, T. Chiba, R. Ozawa, M. Yoshida, M. Hattori, and Y. Sakaki. A comprehensive two-hybrid analysis to explore the yeast protein interactome. Proceedings of the National Academy of Sciences of the United States of America, 98(8):4569-74, apr 2001.

[121] W.-H. Jang, S.-H. Jung, and D.-S. Han. A computational model for predicting protein interactions based on multidomain collaboration. IEEE/ACM transactions on computational biology and bioinformatics / IEEE, ACM, 9(4):1081-90, 2012 .

[122] J. Janin. Welcome to CAPRI: A Critical Assessment of PRedicted Interactions. Proteins: Structure, Function, and Genetics, 47(3):257-257, may 2002.

[123] J. Janin, K. Henrick, J. Moult, L. T. Eyck, M. J. E. Sternberg, S. Vajda, I. Vakser, S. J. Wodak, and Critical Assessment of PRedicted Interactions. CAPRI: a Critical Assessment of PRedicted Interactions. Proteins, 52(1):2-9, jul 2003.

[124] R. Jansen, H. Yu, D. Greenbaum, Y. Kluger, N. J. Krogan, S. Chung, A. Emili, M. Snyder, J. F. Greenblatt, and M. Gerstein. A Bayesian networks approach for predicting protein-protein interactions from genomic data. Science (New York, N.Y.), 302(5644):449-53, oct 2003. 
[125] H. Jeong, S. P. Mason, a. L. Barabási, and Z. N. Oltvai. Lethality and centrality in protein networks. Nature, 411(6833):41-42, may 2001.

[126] M. Jessulat, S. Pitre, Y. Gui, M. Hooshyar, K. Omidi, B. Samanfar, L. H. Tan, M. Alamgir, J. R. Green, F. Dehne, and A. Golshani. Recent advances in protein-protein interaction prediction: experimental and computational methods. Expert opinion on drug discovery, 6(9):921-35, sep 2011.

[127] D. Jin, B. Yang, C. Baquero, D. Liu, D. He, and J. Liu. A Markov random walk under constraint for discovering overlapping communities in complex networks. Journal of Statistical Mechanics: Theory and Experiment, 2011(05):P05031, may 2011.

[128] D. Jones. THREADER: protein sequence threading by double dynamic programming. In Computational Methods in Molecular Biology, volume 32, chapter 13, pages 285-311. Elsevier B.V., Amsterdam; New York, 1998.

[129] D. T. Jones, W. R. Taylor, and J. M. Thornton. A new approach to protein fold recognition. Nature, 358(6381):86-9, jul 1992.

[130] I. K. Jordan, L. S. Katz, D. R. Denver, and J. T. Streelman. Natural selection governs local, but not global, evolutionary gene coexpression networks in Caenorhabditis elegans. BMC systems biology, 2:96, jan 2008.

[131] M. Kamada, Y. Sakuma, M. Hayashida, and T. Akutsu. Prediction of proteinprotein interaction strength using domain features with supervised regression. TheScientific WorldJournal, 2014:240673, jan 2014.

[132] S.-g. Kang and J. G. Saven. Computational protein design: structure, function and combinatorial diversity. Current opinion in chemical biology, 11(3):329-34, jun 2007.

[133] T. S. Keshava Prasad, R. Goel, K. Kandasamy, S. Keerthikumar, S. Kumar, S. Mathivanan, D. Telikicherla, R. Raju, B. Shafreen, A. Venugopal, L. Balakrishnan, A. Marimuthu, S. Banerjee, D. S. Somanathan, A. Sebastian, S. Rani, S. Ray, C. J. Harrys Kishore, S. Kanth, M. Ahmed, M. K. Kashyap, R. Mohmood, Y. L. Ramachandra, V. Krishna, B. A. Rahiman, S. Mohan, P. Ranganathan, S. Ramabadran, R. Chaerkady, and A. Pandey. Human Protein Reference Database-2009 update. Nucleic acids research, 37(Database issue):D767-72, jan 2009.

[134] W. K. Kim, J. Park, and J. K. Suh. Large scale statistical prediction of proteinprotein interaction by potentially interacting domain (PID) pair. Genome informatics. International Conference on Genome Informatics, 13:42-50, jan 2002.

[135] M. Kimura. Evolutionary rate at the molecular level. Nature, 217(5129):624-6, feb 1968. 
[136] M. Kimura. The Neutral Theory of Molecular Evolution. Cambridge University Press, Cambridge, 1983.

[137] L. N. Kinch, W. Li, B. Monastyrskyy, A. Kryshtafovych, and N. V. Grishin. Evaluation of free modeling targets in CASP11 and ROLL. Proteins: Structure, Function, and Bioinformatics, (September):n/a-n/a, 2015.

[138] D. L. Kirk. Volvox: Molecular-Genetic Origins of Multicellularity and Cellular Differentiation. Cabridge University Press, New York, 1998.

[139] D. L. Kirk. A twelve-step program for evolving multicellularity and a division of labor. BioEssays : news and reviews in molecular, cellular and developmental biology, 27(3):299-310, mar 2005.

[140] M. Kirschner and J. Gerhart. Evolvability. Proceedings of the National Academy of Sciences of the United States of America, 95(15):8420-7, jul 1998.

[141] C. G. Knight and J. W. Pinney. Making the right connections: biological networks in the light of evolution. BioEssays : news and reviews in molecular, cellular and developmental biology, 31(10):1080-90, oct 2009.

[142] C. G. Knight, N. Zitzmann, S. Prabhakar, R. Antrobus, R. Dwek, H. Hebestreit, and P. B. Rainey. Unraveling adaptive evolution: how a single point mutation affects the protein coregulation network. Nature genetics, 38(9):1015-22, sep 2006 .

[143] N. J. Krogan, G. Cagney, H. Yu, G. Zhong, X. Guo, A. Ignatchenko, J. Li, S. Pu, N. Datta, A. P. Tikuisis, T. Punna, J. M. Peregrín-Alvarez, M. Shales, X. Zhang, M. Davey, M. D. Robinson, A. Paccanaro, J. E. Bray, A. Sheung, B. Beattie, D. P. Richards, V. Canadien, A. Lalev, F. Mena, P. Wong, A. Starostine, M. M. Canete, J. Vlasblom, S. Wu, C. Orsi, S. R. Collins, S. Chandran, R. Haw, J. J. Rilstone, K. Gandi, N. J. Thompson, G. Musso, P. St Onge, S. Ghanny, M. H. Y. Lam, G. Butland, A. M. Altaf-Ul, S. Kanaya, A. Shilatifard, E. O'Shea, J. S. Weissman, C. J. Ingles, T. R. Hughes, J. Parkinson, M. Gerstein, S. J. Wodak, A. Emili, and J. F. Greenblatt. Global landscape of protein complexes in the yeast Saccharomyces cerevisiae. Nature, 440(7084):637-43, mar 2006.

[144] A. Kryshtafovych, K. Fidelis, and J. Moult. CASP10 results compared to those of previous CASP experiments. Proteins, 82 Suppl 2(0 2):164-74, feb 2014.

[145] A. Kryshtafovych, J. Moult, A. Baslé, A. Burgin, T. K. Craig, R. A. Edwards, D. Fass, M. D. Hartmann, M. Korycinski, R. J. Lewis, D. Lorimer, A. N. Lupas, J. Newman, T. S. Peat, K. H. Piepenbrink, J. Prahlad, M. J. van Raaij, F. Rohwer, A. M. Segall, V. Seguritan, E. J. Sundberg, A. K. Singh, M. A. Wilson, and T. Schwede. Some of the most interesting CASP11 targets through the eyes of their authors. Proteins, (October):n/a-n/a, oct 2015. 
[146] P. Lamesch, T. Z. Berardini, D. Li, D. Swarbreck, C. Wilks, R. Sasidharan, R. Muller, K. Dreher, D. L. Alexander, M. Garcia-Hernandez, A. S. Karthikeyan, C. H. Lee, W. D. Nelson, L. Ploetz, S. Singh, A. Wensel, and E. Huala. The Arabidopsis Information Resource (TAIR): improved gene annotation and new tools. Nucleic acids research, 40(Database issue):D1202-10, jan 2012.

[147] A. Lancichinetti, S. Fortunato, and J. Kertész. Detecting the overlapping and hierarchical community structure in complex networks. New Journal of Physics, 11(3):033015, mar 2009.

[148] A. Lancichinetti, F. Radicchi, J. J. Ramasco, and S. Fortunato. Finding statistically significant communities in networks. PloS one, 6(4):e18961, jan 2011.

[149] M. a. Langston, A. D. Perkins, A. M. Saxton, J. a. Scharff, and B. H. Voy. Innovative Computational Methods for Transcriptomic Data Analysis: A Case Study in the Use of FPT for Practical Algorithm Design and Implementation. The Computer Journal, 51(1):26-38, mar 2007.

[150] A. M. Larracuente, T. B. Sackton, A. J. Greenberg, A. Wong, N. D. Singh, D. Sturgill, Y. Zhang, B. Oliver, and A. G. Clark. Evolution of protein-coding genes in Drosophila. Trends in genetics : TIG, 24(3):114-23, mar 2008.

[151] C. Lee, F. Reid, A. McDaid, and N. Hurley. Detecting highly overlapping community structure by greedy clique expansion. The 16th ACM SIGKDD International Conference on Knowledge Discovery and Data Mining (KDD 2010), 10:10, feb 2010.

[152] S.-A. Lee, C.-h. Chan, C.-H. Tsai, J.-M. Lai, F.-S. Wang, C.-Y. Kao, and C.-Y. F. Huang. Ortholog-based protein-protein interaction prediction and its application to inter-species interactions. BMC bioinformatics, 9 Suppl 12:S11, jan 2008.

[153] J. Leskovec, K. J. Lang, and M. Mahoney. Empirical comparison of algorithms for network community detection. In Proceedings of the 19th international conference on World wide web - $W W W$ '10, page 631, New York, New York, USA, 2010. ACM Press.

[154] E. D. Levy and J. B. Pereira-Leal. Evolution and dynamics of protein interactions and networks. Current opinion in structural biology, 18(3):349-57, jun 2008 .

[155] S. M. Lewis and B. a. Kuhlman. Anchored design of protein-protein interfaces. PLOS ONE, 6(6), 2011.

[156] B. Li and D. Kihara. Protein docking prediction using predicted protein-protein interface. BMC bioinformatics, 13(1):7, jan 2012. 
[157] W. Li and A. Godzik. Cd-hit: a fast program for clustering and comparing large sets of protein or nucleotide sequences. Bioinformatics (Oxford, England), 22(13):1658-9, jul 2006.

[158] L. Licamele and L. Getoor. Predicting Protein-Protein Interactions Using Relational Features. Technical report, UM Computer Science Department, 2007.

[159] X. Lin and X.-w. Chen. Heterogeneous data integration by tree-augmented naïve Bayes for protein-protein interactions prediction. Proteomics, 13(2):2618, jan 2013.

[160] X. Liu, B. Liu, Z. Huang, T. Shi, Y. Chen, and J. Zhang. SPPS: a sequencebased method for predicting probability of protein-protein interaction partners. PloS one, 7(1):e30938, jan 2012.

[161] Y. Liu, I. Chakroun, D. Yang, E. Horner, J. Liang, A. Aziz, A. Chu, Y. De Repentigny, F. J. Dilworth, R. Kothary, and A. Blais. Six1 regulates MyoD expression in adult muscle progenitor cells. PloS one, 8(6):e67762, jun 2013.

[162] Y. Liu, A. Chu, I. Chakroun, U. Islam, and A. Blais. Cooperation between myogenic regulatory factors and SIX family transcription factors is important for myoblast differentiation. Nucleic acids research, 38(20):6857-71, nov 2010.

[163] Z.-p. Liu and L. Chen. Proteome-wide prediction of protein-protein interactions from high-throughput data. Protein \& cell, 3(7):508-20, jul 2012.

[164] Z.-P. Liu, J. Wang, Y.-Q. Qiu, R. K. K. Leung, X.-S. Zhang, S. K. W. Tsui, and L. Chen. Inferring a protein interaction map of Mycobacterium tuberculosis based on sequences and interologs. BMC bioinformatics, 13 Suppl 7(Suppl 7):S6, jan 2012.

[165] S. L. Lo, C. Z. Cai, Y. Z. Chen, and M. C. M. Chung. Effect of training datasets on support vector machine prediction of protein-protein interactions. Proteomics, 5(4):876-84, mar 2005.

[166] M. López-Malo, A. Querol, and J. M. Guillamon. Metabolomic Comparison of Saccharomyces cerevisiae and the Cryotolerant Species S. bayanus var. uvarum and S. kudriavzevii during Wine Fermentation at Low Temperature. PLoS ONE, 8(3):e60135, mar 2013.

[167] L. Lu, H. Lu, and J. Skolnick. MULTIPROSPECTOR: an algorithm for the prediction of protein-protein interactions by multimeric threading. Proteins, 49(3):350-64, nov 2002.

[168] L. J. Lu, Y. Xia, A. Paccanaro, H. Yu, and M. Gerstein. Assessing the limits of genomic data integration for predicting protein networks. Genome research, 15(7):945-53, jul 2005. 
[169] X. Luo, Z. You, M. Zhou, S. Li, H. Leung, Y. Xia, and Q. Zhu. A highly efficient approach to protein interactome mapping based on collaborative filtering framework. Scientific reports, 5:7702, 2015.

[170] Z. Ma, C. Zhou, L. Lu, Y. Ma, P. Sun, and Y. Cui. Predicting protein-protein interactions based on BP neural network. In 2007 IEEE International Conference on Bioinformatics and Biomedicine Workshops, pages 3-7. IEEE, nov 2007.

[171] S. R. Maetschke, M. Simonsen, M. J. Davis, and M. a. Ragan. Gene Ontologydriven inference of protein-protein interactions using inducers. Bioinformatics (Oxford, England), 28(1):69-75, jan 2012.

[172] P. Magwene. Integration and Modularity in Biological System: A Review. Acta Zoologica Sinica, 52(1947):490-493, 2006.

[173] J. G. Mandell, A. Baerga-Ortiz, A. M. Falick, and E. a. Komives. Measurement of Solvent Accessibility at ProteinProtein Interfaces. In Protein-Ligand Interactions, volume 305, pages 065-080. Humana Press, New Jersey, 2005.

[174] E. M. Marcotte, M. Pellegrini, H. L. Ng, D. W. Rice, T. O. Yeates, and D. Eisenberg. Detecting protein function and protein-protein interactions from genome sequences. Science (New York, N.Y.), 285(5428):751-3, jul 1999.

[175] E. M. Marcotte, M. Pellegrini, M. J. Thompson, T. O. Yeates, and D. Eisenberg. A combined algorithm for genome-wide prediction of protein function. Nature, 402(6757):83-6, nov 1999.

[176] S. Martin, D. Roe, and J.-L. Faulon. Predicting protein-protein interactions using signature products. Bioinformatics (Oxford, England), 21(2):218-26, jan 2005 .

[177] Y. Matsuzaki, N. Uchikoga, M. Ohue, T. Shimoda, T. Sato, T. Ishida, and Y. Akiyama. MEGADOCK 3.0: a high-performance protein-protein interaction prediction software using hybrid parallel computing for petascale supercomputing environments. Source code for biology and medicine, 8(1):18, jan 2013.

[178] Y. P. Maturano, M. V. Mestre, B. Esteve-Zarzoso, M. C. Nally, M. C. Lerena, M. E. Toro, F. Vazquez, and M. Combina. Yeast population dynamics during prefermentative cold soak of Cabernet Sauvignon and Malbec wines. International journal of food microbiology, 199:23-32, 2015.

[179] S. S. Merchant, S. E. Prochnik, O. Vallon, E. H. Harris, S. J. Karpowicz, G. B. Witman, A. Terry, A. Salamov, L. K. Fritz-Laylin, L. Maréchal-Drouard, W. F. Marshall, L.-H. Qu, D. R. Nelson, A. A. Sanderfoot, M. H. Spalding, V. V. Kapitonov, Q. Ren, P. Ferris, E. Lindquist, H. Shapiro, S. M. Lucas, J. Grimwood, J. Schmutz, P. Cardol, H. Cerutti, G. Chanfreau, C.-L. Chen, V. Cognat, 
M. T. Croft, R. Dent, S. Dutcher, E. Fernández, H. Fukuzawa, D. GonzálezBallester, D. González-Halphen, A. Hallmann, M. Hanikenne, M. Hippler, W. Inwood, K. Jabbari, M. Kalanon, R. Kuras, P. A. Lefebvre, S. D. Lemaire, A. V. Lobanov, M. Lohr, A. Manuell, I. Meier, L. Mets, M. Mittag, T. Mittelmeier, J. V. Moroney, J. Moseley, C. Napoli, A. M. Nedelcu, K. Niyogi, S. V. Novoselov, I. T. Paulsen, G. Pazour, S. Purton, J.-P. Ral, D. M. RiañoPachón, W. Riekhof, L. Rymarquis, M. Schroda, D. Stern, J. Umen, R. Willows, N. Wilson, S. L. Zimmer, J. Allmer, J. Balk, K. Bisova, C.-J. Chen, M. Elias, K. Gendler, C. Hauser, M. R. Lamb, H. Ledford, J. C. Long, J. Minagawa, M. D. Page, J. Pan, W. Pootakham, S. Roje, A. Rose, E. Stahlberg, A. M. Terauchi, P. Yang, S. Ball, C. Bowler, C. L. Dieckmann, V. N. Gladyshev, P. Green, R. Jorgensen, S. Mayfield, B. Mueller-Roeber, S. Rajamani, R. T. Sayre, P. Brokstein, I. Dubchak, D. Goodstein, L. Hornick, Y. W. Huang, J. Jhaveri, Y. Luo, D. Martínez, W. C. A. Ngau, B. Otillar, A. Poliakov, A. Porter, L. Szajkowski, G. Werner, K. Zhou, I. V. Grigoriev, D. S. Rokhsar, and A. R. Grossman. The Chlamydomonas genome reveals the evolution of key animal and plant functions. Science (New York, N.Y.), 318(5848):245-250, oct 2007.

[180] H. W. Mewes, D. Frishman, C. Gruber, B. Geier, D. Haase, A. Kaps, K. Lemcke, G. Mannhaupt, F. Pfeiffer, C. Schüller, S. Stocker, and B. Weil. MIPS: a database for genomes and protein sequences. Nucleic acids research, 28(1):3740, jan 2000.

[181] R. E. Michod. Darwinian Dynamics: Evolutionary Transitions in Fitness and Individuality. Princeton University Press, 2000.

[182] S. M. Miller. Volvox, Chlamydomonas, and the evolution of multicellularity. Nature Education, 3:65, 2010.

[183] S. M. Miller and D. L. Kirk. glsA, a Volvox gene required for asymmetric division and germ cell specification, encodes a chaperone-like protein. Development (Cambridge, England), 126(4):649-58, feb 1999.

[184] A. Mitchell, H.-Y. Chang, L. Daugherty, M. Fraser, S. Hunter, R. Lopez, C. McAnulla, C. McMenamin, G. Nuka, S. Pesseat, A. Sangrador-Vegas, M. Scheremetjew, C. Rato, S.-Y. Yong, A. Bateman, M. Punta, T. K. Attwood, C. J. A. Sigrist, N. Redaschi, C. Rivoire, I. Xenarios, D. Kahn, D. Guyot, P. Bork, I. Letunic, J. Gough, M. Oates, D. Haft, H. Huang, D. A. Natale, C. H. Wu, C. Orengo, I. Sillitoe, H. Mi, P. D. Thomas, and R. D. Finn. The InterPro protein families database: the classification resource after 15 years. Nucleic acids research, 43(Database issue):D213-21, jan 2015.

[185] T. P. Mohamed, J. G. Carbonell, and M. K. Ganapathiraju. Active learning for human protein-protein interaction prediction. BMC bioinformatics, 11 Suppl 1:S57, jan 2010. 
[186] D. Montarras. Direct Isolation of Satellite Cells for Skeletal Muscle Regeneration. Science, 309(5743):2064-2067, sep 2005.

[187] J. Moult, K. Fidelis, A. Kryshtafovych, T. Schwede, and A. Tramontano. Critical assessment of methods of protein structure prediction (CASP)-round $\mathrm{x}$. Proteins, 82 Suppl 2(0 2):1-6, feb 2014.

[188] J. Moult, J. T. Pedersen, R. Judson, and K. Fidelis. A large-scale experiment to assess protein structure prediction methods. Proteins, 23(3):ii-v, nov 1995.

[189] R. Mrowka, A. Patzak, and H. Herzel. Is there a bias in proteome research? Genome research, 11(12):1971-3, dec 2001.

[190] Y. Murakami and K. Mizuguchi. Homology-based prediction of interactions between proteins using Averaged One-Dependence Estimators. BMC bioinformatics, 15(1):213, 2014.

[191] H. S. Najafabadi and R. Salavati. Sequence-based prediction of protein-protein interactions by means of codon usage. Genome biology, 9(5):R87, jan 2008.

[192] L. Nanni and A. Lumini. An ensemble of K-local hyperplanes for predicting protein-protein interactions. Bioinformatics (Oxford, England), 22(10):120710, may 2006.

[193] M. Nei, Y. Suzuki, and M. Nozawa. The neutral theory of molecular evolution in the genomic era. Annual review of genomics and human genetics, 11:265-89, jan 2010.

[194] T. Nepusz, H. Yu, and A. Paccanaro. Detecting overlapping protein complexes in protein-protein interaction networks. Nature methods, 9(5):471-2, may 2012.

[195] S.-K. Ng, Z. Zhang, and S.-H. Tan. Integrative approach for computationally inferring protein domain interactions. Bioinformatics (Oxford, England), 19(8):923-9, may 2003.

[196] S.-K. Ng, Z. Zhang, S.-H. Tan, and K. Lin. InterDom: a database of putative interacting protein domains for validating predicted protein interactions and complexes. Nucleic acids research, 31(1):251-4, jan 2003.

[197] I. Nishii, S. Ogihara, and D. L. Kirk. A Kinesin, InvA, Plays an Essential Role in Volvox Morphogenesis. Cell, 113(6):743-753, jun 2003.

[198] H. Nordberg, M. Cantor, S. Dusheyko, S. Hua, A. Poliakov, I. Shabalov, T. Smirnova, I. V. Grigoriev, and I. Dubchak. The genome portal of the Department of Energy Joint Genome Institute: 2014 updates. Nucleic acids research, 42(Database issue):D26-31, jan 2014. 
[199] R. Nussinov, R. Sharan, and I. Cohen-Gihon. Rearrangements and Expansion of the Domain Content in Proteins Frequently Increase the Protein Connectivity in the ProteinProtein Interaction Network. In R. Nussinov and G. Schreiber, editors, Computational Protein-Protein Interactions, chapter thesis, pages 211222. CRC Press, jun 2009.

[200] U. Ogmen, O. Keskin, a. S. Aytuna, R. Nussinov, and A. Gursoy. PRISM: protein interactions by structural matching. Nucleic Acids Research, 33(Web Server):W331-W336, jul 2005.

[201] M. Ohue, Y. Matsuzaki, T. Shimoda, T. Ishida, and Y. Akiyama. Highly precise protein-protein interaction prediction based on consensus between templatebased and de novo docking methods. BMC proceedings, 7(Suppl 7):S6, dec 2013 .

[202] K. Omidi, M. Hooshyar, M. Jessulat, B. Samanfar, M. Sanders, D. Burnside, S. Pitre, A. Schoenrock, J. Xu, M. Babu, and A. Golshani. Phosphatase complex Pph3/Psy2 is involved in regulation of efficient non-homologous end-joining pathway in the yeast Saccharomyces cerevisiae. PloS one, 9(1):e87248, jan 2014.

[203] R. Overbeek, M. Fonstein, M. D'Souza, G. D. Pusch, and N. Maltsev. The use of gene clusters to infer functional coupling. Proceedings of the National Academy of Sciences of the United States of America, 96(6):2896-901, mar 1999.

[204] A. Ozgür, T. Vu, G. Erkan, and D. R. Radev. Identifying gene-disease associations using centrality on a literature mined gene-interaction network. Bioinformatics (Oxford, England), 24(13):i277-85, jul 2008.

[205] G. Palla, I. Derényi, I. Farkas, and T. Vicsek. Uncovering the overlapping community structure of complex networks in nature and society. Nature, 435(7043):814-8, jun 2005.

[206] X.-Y. Pan, Y.-N. Zhang, and H.-B. Shen. Large-scale prediction of human protein-protein interactions from amino acid sequence based on latent topic features. Journal of proteome research, 9(10):4992-5001, oct 2010.

[207] R. J. Pantazes, M. J. Grisewood, and C. D. Maranas. Recent advances in computational protein design. Current opinion in structural biology, 21(4):46772 , aug 2011.

[208] B. Papp, R. A. Notebaart, and C. Pál. Systems-biology approaches for predicting genomic evolution. Nature reviews. Genetics, 12(9):591-602, sep 2011.

[209] M. S. Paradesi, D. Caragea, and W. H. Hsu. Structural Prediction of ProteinProtein Interactions in Saccharomyces cerevisiae. In 2007 IEEE 7th International Symposium on BioInformatics and BioEngineering, pages 1270-1274. IEEE, oct 2007. 
[210] Y. Park. Critical assessment of sequence-based protein-protein interaction prediction methods that do not require homologous protein sequences. BMC bioinformatics, 10:419, jan 2009.

[211] Y. Park and E. M. Marcotte. Revisiting the negative example sampling problem for predicting protein-protein interactions. Bioinformatics (Oxford, England), 27(21):3024-8, nov 2011.

[212] C. Patulea. Targeted Optimization of Computational and Classification Performance of a Protein-Protein Interaction Predictor. Master of applied science, Carleton University, 2011.

[213] F. Pazos and A. Valencia. Similarity of phylogenetic trees as indicator of proteinprotein interaction. Protein Engineering Design and Selection, 14(9):609-614, sep 2001.

[214] F. Pazos and A. Valencia. In silico two-hybrid system for the selection of physically interacting protein pairs. Proteins, 47(2):219-27, may 2002.

[215] P. Pei and A. Zhang. A topological measurement for weighted protein interaction network. Proceedings / IEEE Computational Systems Bioinformatics Conference, CSB. IEEE Computational Systems Bioinformatics Conference, pages 268-78, jan 2005.

[216] M. Pellegrini, E. M. Marcotte, M. J. Thompson, D. Eisenberg, and T. O. Yeates. Assigning protein functions by comparative genome analysis: protein phylogenetic profiles. Proceedings of the National Academy of Sciences of the United States of America, 96(8):4285-8, apr 1999.

[217] J. M. Peltier, S. Askovic, R. R. Becklin, C. L. Chepanoske, Y.-S. J. Ho, V. Kery, S. Lai, T. Mujtaba, M. Pyne, P. B. Robbins, M. V. Rechenberg, B. Richardson, J. Savage, P. Sheffield, S. Thompson, L. Weir, K. Widjaja, N. Xu, Y. Zhen, and J. J. Boniface. An integrated strategy for the discovery of drug targets by the analysis of proteinprotein interactions. International Journal of Mass Spectrometry, 238(2):119-130, nov 2004.

[218] A. Pérez-Bercoff, C. M. Hudson, and G. C. Conant. A conserved mammalian protein interaction network. PloS one, 8(1):e52581, jan 2013.

[219] J. Petschnigg, B. Groisman, M. Kotlyar, M. Taipale, Y. Zheng, C. F. Kurat, A. Sayad, J. R. Sierra, M. M. Usaj, J. Snider, A. Nachman, I. Krykbaeva, M.-S. Tsao, J. Moffat, T. Pawson, S. Lindquist, I. Jurisica, and I. Stagljar. The mammalian-membrane two-hybrid assay (MaMTH) for probing membraneprotein interactions in human cells. Nature methods, 11(5):585-92, 2014.

[220] A. Philpott and D. J. Winton. Lineage selection and plasticity in the intestinal crypt. Current Opinion in Cell Biology, 31:39-45, dec 2014. 
[221] M. Pigliucci. Is evolvability evolvable? Nature reviews. Genetics, 9(1):75-82, jan 2008.

[222] M. Pigliucci and K. Preston, editors. Phenotypic Integration: Studying the Ecology and Evolution of Complex Phenotypes. Oxford University Press, 2004.

[223] S. Pitre, M. Alamgir, J. R. Green, M. Dumontier, F. Dehne, and A. Golshani. Computational Methods For Predicting ProteinProtein Interactions. In Advances in Biochemical Engineering/Biotechnology. Springer Berlin / Heidelberg, 2008 .

[224] S. Pitre, F. Dehne, A. Chan, J. Cheetham, A. Duong, A. Emili, M. Gebbia, J. Greenblatt, M. Jessulat, N. Krogan, X. Luo, and A. Golshani. PIPE: a protein-protein interaction prediction engine based on the re-occurring short polypeptide sequences between known interacting protein pairs. BMC bioinformatics, 7:365, jan 2006 .

[225] S. Pitre, M. Hooshyar, A. Schoenrock, B. Samanfar, M. Jessulat, J. R. Green, F. Dehne, and A. Golshani. Short Co-occurring Polypeptide Regions Can Predict Global Protein Interaction Maps. Scientific reports, 2:239, jan 2012.

[226] S. Pitre, C. North, M. Alamgir, M. Jessulat, A. Chan, X. Luo, J. R. Green, M. Dumontier, F. Dehne, and A. Golshani. Global investigation of proteinprotein interactions in yeast Saccharomyces cerevisiae using re-occurring short polypeptide sequences. Nucleic acids research, 36(13):4286-94, aug 2008.

[227] S. E. Prochnik, J. Umen, A. M. Nedelcu, A. Hallmann, S. M. Miller, I. Nishii, P. Ferris, A. Kuo, T. Mitros, L. K. Fritz-Laylin, U. Hellsten, J. Chapman, O. Simakov, S. a. Rensing, A. Terry, J. Pangilinan, V. Kapitonov, J. Jurka, A. Salamov, H. Shapiro, J. Schmutz, J. Grimwood, E. Lindquist, S. Lucas, I. V. Grigoriev, R. Schmitt, D. Kirk, and D. S. Rokhsar. Genomic analysis of organismal complexity in the multicellular green alga Volvox carteri. Science (New York, N.Y.), 329(5988):223-6, jul 2010.

[228] Y. Qi, Z. Bar-Joseph, and J. Klein-Seetharaman. Evaluation of different biological data and computational classification methods for use in protein interaction prediction. Proteins, 63(3):490-500, may 2006.

[229] Y. Qi, J. Klein-Seetharaman, and Z. Bar-Joseph. Random forest similarity for protein-protein interaction prediction from multiple sources. Pacific Symposium on Biocomputing. Pacific Symposium on Biocomputing, 542:531-42, jan 2005.

[230] Y. Qi, J. Klein-Seetharaman, and Z. Bar-Joseph. A mixture of feature experts approach for protein-protein interaction prediction. BMC bioinformatics, 8 Suppl 10(Suppl 10):S6, 2007.

[231] R Core Team. R: A language and environment for statistical computing, 2014. 
[232] B. Raghavachari, A. Tasneem, T. M. Przytycka, and R. Jothi. DOMINE: a database of protein domain interactions. Nucleic acids research, 36(Database issue):D656-61, 2008.

[233] J. Reimand, S. Hui, S. Jain, B. Law, and G. D. Bader. Domain-mediated protein interaction prediction: From genome to network. FEBS letters, 586(17):275163, aug 2012.

[234] X. Ren, Y.-C. Wang, Y. Wang, X.-S. Zhang, and N.-Y. Deng. Improving accuracy of protein-protein interaction prediction by considering the converse problem for sequence representation. BMC bioinformatics, 12(1):409, jan 2011.

[235] G. Rigaut, A. Shevchenko, B. Rutz, M. Wilm, M. Mann, and B. Séraphin. A generic protein purification method for protein complex characterization and proteome exploration. Nature biotechnology, 17(10):1030-2, oct 1999.

[236] R. Riley, C. Lee, C. Sabatti, and D. Eisenberg. Inferring protein domain interactions from databases of interacting proteins. Genome biology, 6(10):R89, 2005 .

[237] E. Rodgers-Melnick, M. Culp, and S. P. DiFazio. Predicting whole genome protein interaction networks from primary sequence data in model and nonmodel organisms using ENTS. BMC genomics, 14:608, 2013.

[238] A. Roguev, S. Bandyopadhyay, M. Zofall, K. Zhang, T. Fischer, S. R. Collins, H. Qu, M. Shales, H.-O. Park, J. Hayles, K.-L. Hoe, D.-U. Kim, T. Ideker, S. I. Grewal, J. S. Weissman, and N. J. Krogan. Conservation and rewiring of functional modules revealed by an epistasis map in fission yeast. Science (New York, N.Y.), 322(5900):405-10, oct 2008.

[239] M. J. Root, M. S. Kay, and P. S. Kim. Protein design of an HIV-1 entry inhibitor. Science (New York, N.Y.), 291(5505):884-8, feb 2001.

[240] S. Roy, D. Martinez, H. Platero, T. Lane, and M. Werner-Washburne. Exploiting amino acid composition for predicting protein-protein interactions. PloS one, 4(11):e7813, jan 2009.

[241] G. M. Rubin, M. D. Yandell, J. R. Wortman, G. L. Gabor Miklos, C. R. Nelson, I. K. Hariharan, M. E. Fortini, P. W. Li, R. Apweiler, W. Fleischmann, J. M. Cherry, S. Henikoff, M. P. Skupski, S. Misra, M. Ashburner, E. Birney, M. S. Boguski, T. Brody, P. Brokstein, S. E. Celniker, S. A. Chervitz, D. Coates, A. Cravchik, A. Gabrielian, R. F. Galle, W. M. Gelbart, R. A. George, L. S. Goldstein, F. Gong, P. Guan, N. L. Harris, B. A. Hay, R. A. Hoskins, J. Li, Z. Li, R. O. Hynes, S. J. Jones, P. M. Kuehl, B. Lemaitre, J. T. Littleton, D. K. Morrison, C. Mungall, P. H. O'Farrell, O. K. Pickeral, C. Shue, L. B. Vosshall, J. Zhang, Q. Zhao, X. H. Zheng, and S. Lewis. Comparative genomics of the eukaryotes. Science (New York, N.Y.), 287(5461):2204-15, mar 2000. 
[242] D. P. Ryan and J. M. Matthews. Protein-protein interactions in human disease. Current opinion in structural biology, 15(4):441-6, aug 2005.

[243] I. Saha, T. Klingström, S. Forsberg, J. Wikander, J. Zubek, M. Kierczak, and D. Plewczynski. Evaluation of Machine Learning Algorithms on Protein-Protein Interactions. In D. A. Gruca, T. Czachórski, and S. Kozielski, editors, ManMachine Interactions 3, volume 242 of Advances in Intelligent Systems and Computing, pages 211-218. Springer International Publishing, Cham, 2014.

[244] I. Saha, J. Zubek, T. Klingström, S. Forsberg, J. Wikander, M. Kierczak, U. Maulik, and D. Plewczynski. Ensemble learning prediction of proteinprotein interactions using proteins functional annotations. Molecular bioSystems, 10(4):820-30, 2014.

[245] R. Saito, H. Suzuki, and Y. Hayashizaki. Interaction generality, a measurement to assess the reliability of a protein-protein interaction. Nucleic acids research, 30(5):1163-8, mar 2002.

[246] R. Saito, H. Suzuki, and Y. Hayashizaki. Construction of reliable proteinprotein interaction networks with a new interaction generality measure. Bioinformatics, 19(6):756-763, apr 2003.

[247] L. Salwinski, C. S. Miller, A. J. Smith, F. K. Pettit, J. U. Bowie, and D. Eisenberg. The Database of Interacting Proteins: 2004 update. Nucleic acids research, 32(Database issue):D449-51, jan 2004.

[248] J. G. Saven. Computational protein design: Advances in the design and redesign of biomolecular nanostructures. Current opinion in colloid $\mathscr{G}$ interface science, 15(1-2):13-17, apr 2010.

[249] D. R. Scannell, O. a. Zill, A. Rokas, C. Payen, M. J. Dunham, M. B. Eisen, J. Rine, M. Johnston, and C. T. Hittinger. The Awesome Power of Yeast Evolutionary Genetics: New Genome Sequences and Strain Resources for the Saccharomyces sensu stricto Genus. G3 (Bethesda, Md.), 1(1):11-25, jun 2011.

[250] A. Schoenrock. PIPE3 : A Massively Parallel Protein-Protein Prediction Engine. Master of computer science, Carleton University, 2010.

[251] A. Schoenrock, D. Burnside, H. Moteshareie, S. Pitre, A. Golshani, F. Dehne, and A. Wong. A study of the Evolution of Protein-Protein Interaction Networks in Yeast. in preparation, to be submitted for publication shortly.

[252] A. Schoenrock, D. Burnside, H. Moteshareie, A. Wong, A. Golshani, F. Dehne, and J. R. Green. Engineering inhibitory proteins with InSiPS: The InSilico Protein Synthesizer. In Proceedings of the International Conference for High Performance Computing, Networking, Storage and Analysis - SC '15, pages 1-11, New York, New York, USA, 2015. ACM Press. 
[253] A. Schoenrock, F. Dehne, J. R. Green, A. Golshani, and S. Pitre. MP-PIPE: A Massively Parallel Protein-Protein Interaction Prediction Engine. In Proceedings of the international conference on Supercomputing - ICS '11, page 327, New York, New York, USA, 2011. ACM Press.

[254] A. Schoenrock, J. Featherston, E. R. Hanschen, T. N. Marriage, T. Hamaji, H. Kawai-Toyooka, M. Suzuki, H. Noguchi, Y. Minakuchi, A. Toyoda, A. Fujiyama, B. J. Olson, H. Nozaki, R. E. Michod, F. Dehne, and P. M. Durand. Protein systems biology of an evolutionary transition in individuality. submitted to Scientific Reports (submission \#: SREP-16-02483-T), jan 2016.

[255] A. Schoenrock, B. Samanfar, S. Pitre, M. Hooshyar, K. Jin, C. a. Phillips, H. Wang, S. Phanse, K. Omidi, Y. Gui, M. Alamgir, A. Wong, F. Barrenäs, M. Babu, M. Benson, M. a. Langston, J. R. Green, F. Dehne, and A. Golshani. Efficient prediction of human protein-protein interactions at a global scale. BMC bioinformatics, 15(1):383, dec 2014.

[256] J. W. Scott, S. A. Hawley, K. A. Green, M. Anis, G. Stewart, G. A. Scullion, D. G. Norman, and D. G. Hardie. CBS domains form energy-sensing modules whose binding of adenosine ligands is disrupted by disease mutations. The Journal of clinical investigation, 113(2):274-84, jan 2004.

[257] R. Sharan, S. Suthram, R. M. Kelley, T. Kuhn, S. McCuine, P. Uetz, T. Sittler, R. M. Karp, and T. Ideker. Conserved patterns of protein interaction in multiple species. Proceedings of the National Academy of Sciences of the United States of America, 102(6):1974-9, mar 2005.

[258] H. Shen, X. Cheng, K. Cai, and M.-b. Hu. Detect overlapping and hierarchical community structure in networks. Physica A: Statistical Mechanics and its Applications, 388(8):1706-1712, apr 2009.

[259] J. Shen, J. Zhang, X. Luo, W. Zhu, K. Yu, K. Chen, Y. Li, and H. Jiang. Predicting protein-protein interactions based only on sequences information. Proceedings of the National Academy of Sciences of the United States of America, 104(11):4337-41, mar 2007.

[260] M.-G. Shi, J.-F. Xia, X.-L. Li, and D.-S. Huang. Predicting protein-protein interactions from sequence using correlation coefficient and high-quality interaction dataset. Amino acids, 38(3):891-9, mar 2010.

[261] B. a. Shoemaker and A. R. Panchenko. Deciphering protein-protein interactions. Part I. Experimental techniques and databases. PLoS computational biology, 3(3):e42, mar 2007.

[262] R. Singh, D. Park, J. Xu, R. Hosur, and B. Berger. Struct2Net: a web service to predict protein-protein interactions using a structure-based approach. Nucleic acids research, 38(Web Server issue):W508-15, jul 2010. 
[263] J. Skolnick and D. Kihara. Defrosting the frozen approximation: PROSPECTOR-a new approach to threading. Proteins, 42(3):319-31, mar 2001.

[264] L. Skrabanek, H. K. Saini, G. D. Bader, and A. J. Enright. Computational prediction of protein-protein interactions. Molecular biotechnology, 38(1):1-17, jan 2008.

[265] G. R. Smith and M. J. E. Sternberg. Prediction of protein-protein interactions by docking methods. Current opinion in structural biology, 12(1):28-35, mar 2002 .

[266] B. Snel, P. Bork, and M. a. Huynen. The identification of functional modules from the genomic association of genes. Proceedings of the National Academy of Sciences of the United States of America, 99(9):5890-5, apr 2002.

[267] E. Sprinzak and H. Margalit. Correlated sequence-signatures as markers of protein-protein interaction. Journal of molecular biology, 311(4):681-92, aug 2001.

[268] A. Stein, R. B. Russell, and P. Aloy. 3Did: Interacting Protein Domains of Known Three-Dimensional Structure. Nucleic acids research, 33(Database issue):D413-7, jan 2005.

[269] J. Tamames, G. Casari, C. Ouzounis, and A. Valencia. Conserved clusters of functionally related genes in two bacterial genomes. Journal of molecular evolution, 44(1):66-73, jan 1997.

[270] F. S. Tedesco and G. Cossu. Stem cell therapies for muscle disorders. Current Opinion in Neurology, 25(5):597-603, oct 2012.

[271] M. C. Teixeira, L. R. Raposo, N. P. Mira, A. B. Lourenço, and I. SáCorreia. Genome-wide identification of Saccharomyces cerevisiae genes required for maximal tolerance to ethanol. Applied and environmental microbiology, 75(18):5761-72, sep 2009.

[272] O. Tenaillon, A. Rodríguez-Verdugo, R. L. Gaut, P. McDonald, A. F. Bennett, A. D. Long, and B. S. Gaut. The molecular diversity of adaptive convergence. Science (New York, N.Y.), 335(6067):457-61, jan 2012.

[273] The Encode Consortium Project. A user's guide to the encyclopedia of DNA elements (ENCODE). PLoS biology, 9(4):e1001046, apr 2011.

[274] The Uniprot Consortium. Activities at the Universal Protein Resource (UniProt). Nucleic acids research, 42(Database issue):D191-8, jan 2014.

[275] S. Tsoka and C. a. Ouzounis. Prediction of protein interactions: metabolic enzymes are frequently involved in gene fusion. Nature genetics, 26(2):141-2, oct 2000 . 
[276] C. Turano, E. Gaucci, C. Grillo, and S. Chichiarelli. ERp57/GRP58: A protein with multiple functions, 2011.

[277] N. Ueki and I. Nishii. Idaten is a new cold-inducible transposon of Volvox carteri that can be used for tagging developmentally important genes. Genetics, 180(3):1343-53, nov 2008.

[278] N. Ueki and I. Nishii. Controlled enlargement of the glycoprotein vesicle surrounding a volvox embryo requires the InvB nucleotide-sugar transporter and is required for normal morphogenesis. The Plant cell, 21(4):1166-81, apr 2009.

[279] P. Uetz, L. Giot, G. Cagney, T. A. Mansfield, R. S. Judson, J. R. Knight, D. Lockshon, V. Narayan, M. Srinivasan, P. Pochart, A. Qureshi-Emili, Y. Li, B. Godwin, D. Conover, T. Kalbfleisch, G. Vijayadamodar, M. Yang, M. Johnston, S. Fields, and J. M. Rothberg. A comprehensive analysis of protein-protein interactions in Saccharomyces cerevisiae. Nature, 403(6770):623-7, feb 2000.

[280] A. Valencia and F. Pazos. Computational methods for the prediction of protein interactions. Current Opinion in Structural Biology, 12(3):368-373, jun 2002.

[281] T. Vellai and G. Vida. The origin of eukaryotes: the difference between prokaryotic and eukaryotic cells. Proceedings. Biological sciences / The Royal Society, 266(1428):1571-1577, 1999.

[282] J.-P. Vert. A tree kernel to analyse phylogenetic profiles. Bioinformatics (Oxford, England), 18 Suppl 1:S276-84, jan 2002.

[283] B. O. Villoutreix, M. a. Kuenemann, J. L. Poyet, H. Bruzzoni-Giovanelli, C. Labbé, D. Lagorce, O. Sperandio, and M. a. Miteva. Drug-like proteinprotein interaction modulators: Challenges and opportunities for drug discovery and chemical biology, 2014.

[284] A. Wagner. Evolutionary constraints permeate large metabolic networks. BMC evolutionary biology, 9:231, jan 2009.

[285] a. J. Walhout. Protein Interaction Mapping in C. elegans Using Proteins Involved in Vulval Development. Science, 287(5450):116-122, jan 2000.

[286] H. Wang, H. Huang, C. Ding, and F. Nie. Predicting protein-protein interactions from multimodal biological data sources via nonnegative matrix trifactorization. Journal of computational biology : a journal of computational molecular cell biology, 20(4):344-58, apr 2013.

[287] Y. Wang, J. Wang, Z. Yang, and N. Deng. Sequence-based protein-protein interaction prediction via support vector machine. Journal of Systems Science and Complexity, 23(5):1012-1023, nov 2010. 
[288] M. N. Wass, G. Fuentes, C. Pons, F. Pazos, and A. Valencia. Towards the prediction of protein interaction partners using physical docking. Molecular systems biology, 7(469):469, mar 2011.

[289] J. Wu, L.-Y. Lu, and X. Yu. The role of BRCA1 in DNA damage response. Protein \& cell, 1(2):117-23, feb 2010.

[290] S. Wuchty. Topology and weights in a protein domain interaction network-a novel way to predict protein interactions. BMC genomics, 7:122, jan 2006.

[291] J.-F. Xia, X.-M. Zhao, and D.-S. Huang. Predicting protein-protein interactions from protein sequences using meta predictor. Amino acids, 39(5):1595-9, nov 2010.

[292] J. Xie, S. Kelley, and B. K. Szymanski. Overlapping community detection in networks: the State of the Art and Comparative Study. ACM Computing Surveys, 45(4):1-35, aug 2013.

[293] J. Xie and B. K. Szymanski. Community Detection Using A Neighborhood Strength Driven Label Propagation Algorithm. Network Science Workshop (NSW), $2011 \ldots, 2011$.

[294] J. Xie and B. K. Szymanski. Towards Linear Time Overlapping Community Detection in Social Networks. Advances in Knowledge Discovery and Data Mining, 7302, feb 2012.

[295] J. Xie and B. K. Szymanski. LabelRank: A stabilized label propagation algorithm for community detection in networks. In 2013 IEEE 2nd Network Science Workshop (NSW), pages 138-143. IEEE, apr 2013.

[296] J. Xie, B. K. Szymanski, and X. Liu. SLPA: Uncovering Overlapping Communities in Social Networks via a Speaker-Listener Interaction Dynamic Process. In 2011 IEEE 11th International Conference on Data Mining Workshops, pages 344-349. IEEE, dec 2011.

[297] H. Yajima, N. Motohashi, Y. Ono, S. Sato, K. Ikeda, S. Masuda, E. Yada, H. Kanesaki, Y. Miyagoe-Suzuki, S. Takeda, and K. Kawakami. Six family genes control the proliferation and differentiation of muscle satellite cells. Experimental cell research, 316(17):2932-44, oct 2010.

[298] L. Yang and X. Tang. Protein-protein interactions prediction based on iterative clique extension with gene ontology filtering. TheScientificWorldJournal, 2014:523634, 2014.

[299] Z. Yang. PAML 4: phylogenetic analysis by maximum likelihood. Molecular biology and evolution, 24(8):1586-91, aug 2007. 
[300] Z.-H. You, K. C. C. Chan, and P. Hu. Predicting protein-protein interactions from primary protein sequences using a novel multi-scale local feature representation scheme and the random forest. PloS one, 10(5):e0125811, may 2015.

[301] Z.-H. You, Y.-K. Lei, J. Gui, D.-S. Huang, and X. Zhou. Using manifold embedding for assessing and predicting protein interactions from high-throughput experimental data. Bioinformatics (Oxford, England), 26(21):2744-51, nov 2010.

[302] Z.-H. You, Y.-K. Lei, L. Zhu, J. Xia, and B. Wang. Prediction of proteinprotein interactions from amino acid sequences with ensemble extreme learning machines and principal component analysis. BMC bioinformatics, 14 Suppl 8(Suppl 8):S10, jan 2013.

[303] Z.-H. You, S. Li, X. Gao, X. Luo, and Z. Ji. Large-scale protein-protein interactions detection by integrating big biosensing data with computational model. BioMed research international, 2014:598129, 2014.

[304] Z.-H. You, J.-Z. Yu, L. Zhu, S. Li, and Z.-K. Wen. A MapReduce based parallel SVM for large-scale predicting proteinprotein interactions. Neurocomputing, 145:37-43, dec 2014.

[305] C.-Y. Yu, L.-C. Chou, and D. T.-H. Chang. Predicting protein-protein interactions in unbalanced data using the primary structure of proteins. $B M C$ bioinformatics, 11:167, jan 2010.

[306] F. Yu, V. M. Cangelosi, M. L. Zastrow, M. Tegoni, J. S. Plegaria, A. G. Tebo, C. S. Mocny, L. Ruckthong, H. Qayyum, and V. L. Pecoraro. Protein design: toward functional metalloenzymes. Chemical reviews, 114(7):3495-578, apr 2014 .

[307] H. Yu, D. Greenbaum, H. Xin Lu, X. Zhu, and M. Gerstein. Genomic analysis of essentiality within protein networks. Trends in genetics : TIG, 20(6):227-31, jun 2004.

[308] H. Yu, P. M. Kim, E. Sprecher, V. Trifonov, and M. Gerstein. The importance of bottlenecks in protein networks: correlation with gene essentiality and expression dynamics. PLoS computational biology, 3(4):e59, apr 2007.

[309] H. Yu, N. M. Luscombe, H. X. Lu, X. Zhu, Y. Xia, J.-D. J. Han, N. Bertin, S. Chung, M. Vidal, and M. Gerstein. Annotation transfer between genomes: protein-protein interologs and protein-DNA regulogs. Genome research, 14(6):1107-18, jun 2004.

[310] J. Yu, M. Guo, C. J. Needham, Y. Huang, L. Cai, and D. R. Westhead. Simple sequence-based kernels do not predict protein-protein interactions. Bioinformatics (Oxford, England), 26(20):2610-4, oct 2010. 
[311] R. Zagozdzon, W. M. Gallagher, and J. Crown. Truncated HER2: implications for HER2-targeted therapeutics. Drug discovery today, 16(17-18):810-6, sep 2011.

[312] J. Zahiri, J. H. Bozorgmehr, and A. Masoudi-Nejad. Computational Prediction of Protein-Protein Interaction Networks: Algorithms and Resources. Current genomics, 14(6):397-414, sep 2013.

[313] N. Zaki, S. Lazarova-Molnar, W. El-Hajj, and P. Campbell. Protein-protein interaction based on pairwise similarity. BMC bioinformatics, 10:150, jan 2009.

[314] A. Zanghellini. de novo computational enzyme design. Current opinion in biotechnology, 29:132-8, oct 2014.

[315] L. V. Zhang, S. L. Wong, O. D. King, and F. P. Roth. Predicting co-complexed protein pairs using genomic and proteomic data integration. BMC bioinformatics, 5:38, apr 2004 .

[316] Q. C. Zhang, D. Petrey, L. Deng, L. Qiang, Y. Shi, C. A. Thu, B. Bisikirska, C. Lefebvre, D. Accili, T. Hunter, T. Maniatis, A. Califano, and B. Honig. Structure-based prediction of protein-protein interactions on a genome-wide scale. Nature, 490(7421):556-60, oct 2012.

[317] S.-W. Zhang, Y.-M. Cheng, L. Luo, and Q. Pan. Prediction of Protein-Protein Interaction Using Distance Frequency of Amino Acids Grouped with their Physicochemical Properties. In 2011 Sixth International Conference on BioInspired Computing: Theories and Applications, pages 70-74. IEEE, sep 2011.

[318] S.-W. Zhang, L.-Y. Hao, and T.-H. Zhang. Prediction of protein-protein interaction with pairwise kernel support vector machine. International journal of molecular sciences, 15(2):3220-33, feb 2014.

[319] Y. Zhou, Y.-S. Zhou, F. He, J. Song, and Z. Zhang. Can simple codon pair usage predict protein-protein interaction? Molecular bioSystems, 8(5):1396-404, apr 2012 .

[320] J. Zubek, M. Tatjewski, A. Boniecki, M. Mnich, S. Basu, and D. Plewczynski. Multi-level machine learning prediction of protein-protein interactions in Saccharomyces cerevisiae. PeerJ, 3:e1041, jul 2015. 